Compound NSC Identifiers as Assigned by the National Cancer Institute.

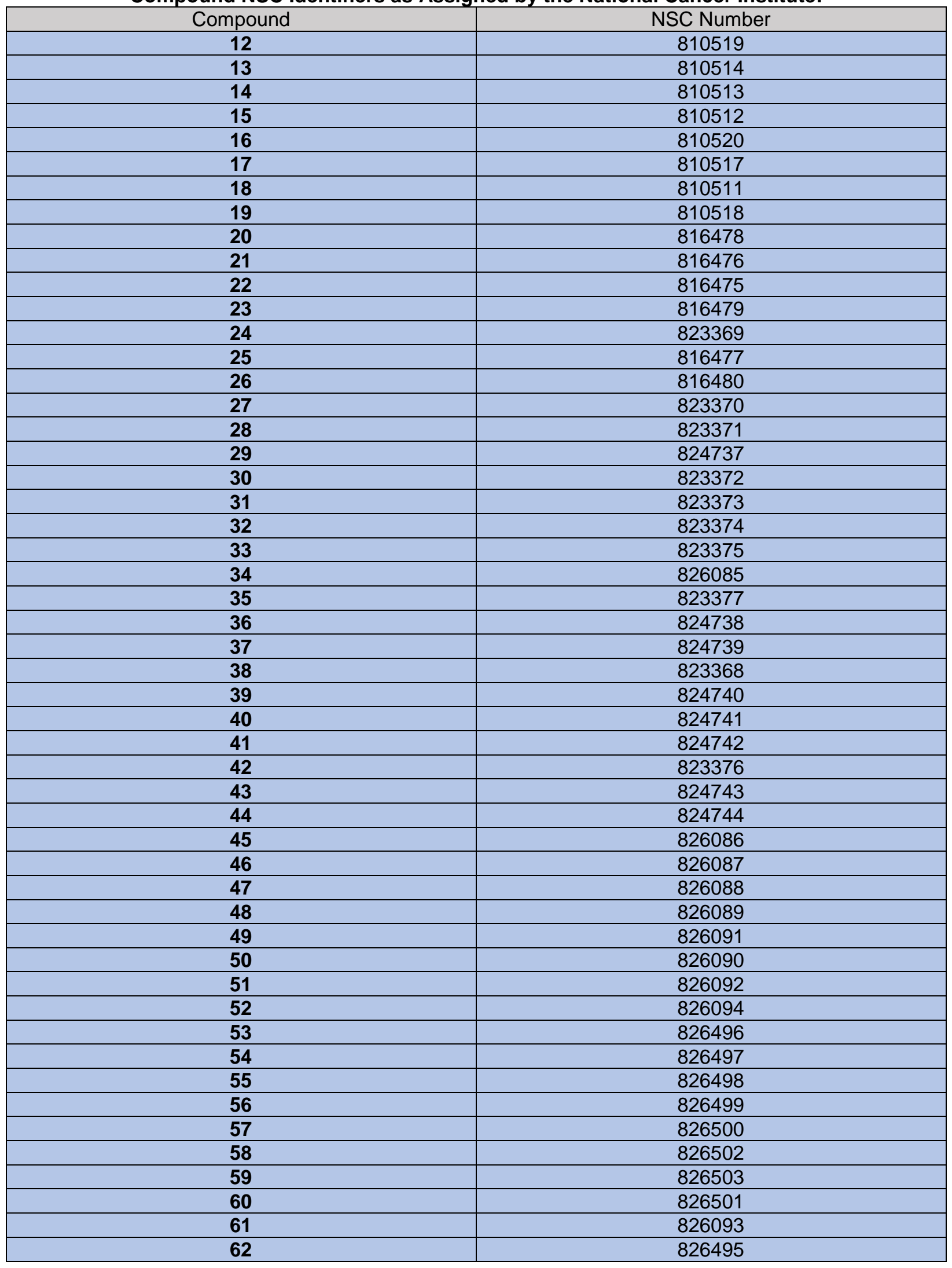


NSC: D-810519/1

\section{Panel/Cell Line}

Leukemia

CCRF-CEM

HL-60(TB)

$\mathrm{K}-562$

MOLT-4

RPMI-8226

$\mathrm{SR}$

Non-Small Cell Lung Cancer

A549/ATCC

EKVX

HOP-62

HOP-92

$\mathrm{NCl}-\mathrm{H} 226$

$\mathrm{NCl}-\mathrm{H} 23$

$\mathrm{NCl}-\mathrm{H} 322 \mathrm{M}$

$\mathrm{NCl}-\mathrm{H} 460$

$\mathrm{NCl}-\mathrm{H} 522$

Colon Cancer

COLO 205

HCC-2998

HCT-116

HCT-15

HT29

KM12

SW-620

CNS Cancer

SF-268

SF-539

SNB-19

SNB-75

U251

Melanoma

LOX IMVI

MALME-3M

M14

MDA-MB-435

SK-MEL-2

SK-MEL-28

SK-MEL-5

UACC-257

UACC-62

Ovarian Cancer

IGROV1

OVCAR-3

OVCAR-4

OVCAR-5

OVCAR-8

NCI/ADR-RES

SK-OV-3

Renal Cancer

786-0

A498

ACHN

CAKI-1

RXF 393

SN12C

TK-10

UO-31

Prostate Cancer

PC-3

DU-145

Breast Cancer

MCF7

MDA-MB-231/ATCC

HS $578 \mathrm{~T}$

BT-549

T-47D

MDA-MB-468

\section{Growth Percent}

10.54

$-28.08$

11.89

12.67

17.16

11.63

37.75

44.45

34.68

50.47

63.92

41.96

52.79

15.80

0.05

20.17

56.37

18.81

19.92

7.98

21.20

20.62

45.46

30.19

19.40

29.69

0.81

21.02

51.45

45.99

12.16

$-10.30$

12.82

56.23

25.52

43.35

27.52

37.87

10.18

69.87

59.09

30.43

$-0.89$

39.74

38.50

14.08

31.98

36.34

25.88

35.47

32.45

36.30

31.25

34.03

16.33

49.65

14.12

52.80

32.61

4.54

28.11

56.19

97.95
Mean Growth Percent - Growth Percent

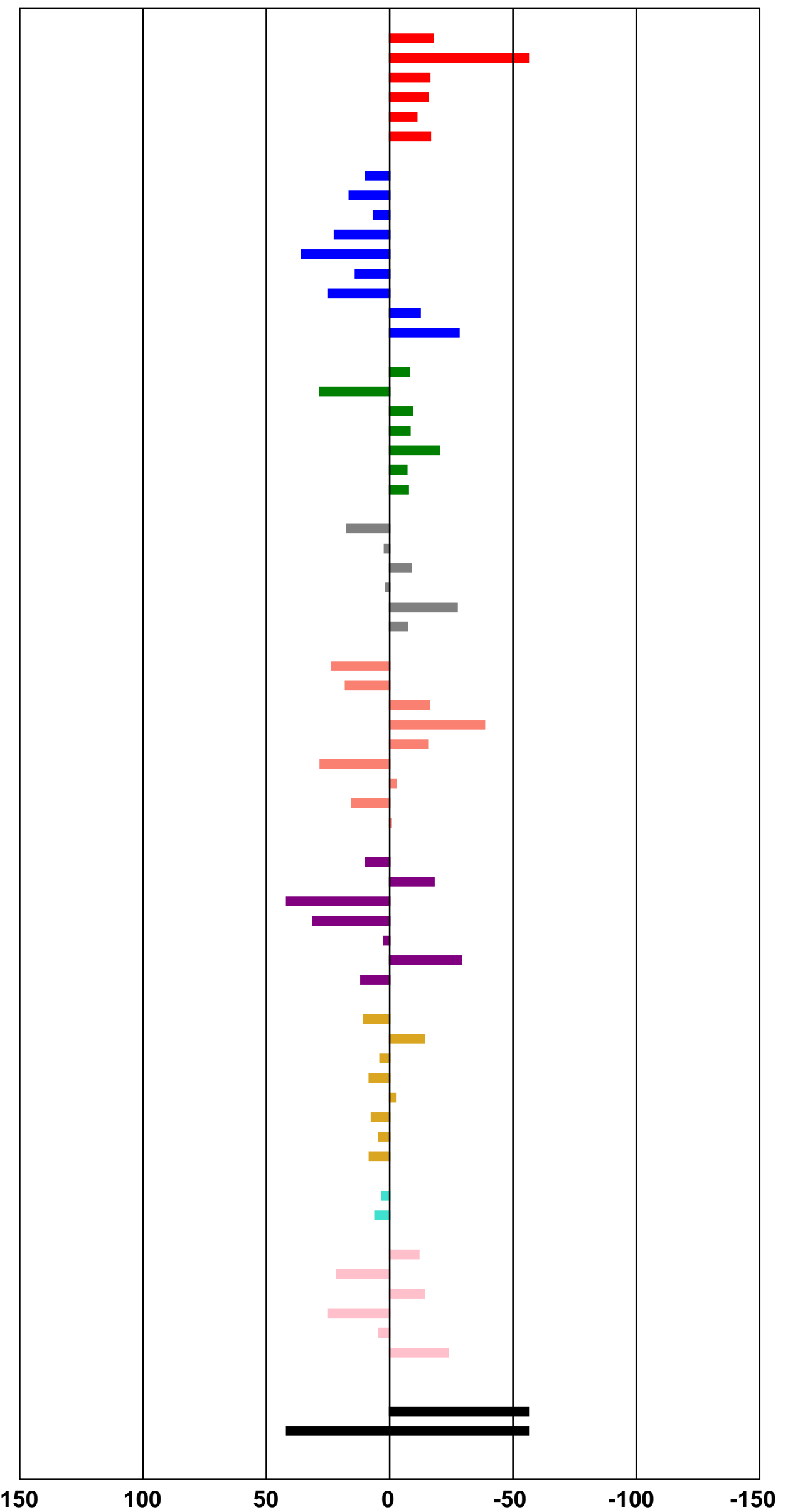


Developmental Therapeutics Program One Dose Mean Graph
NSC: D-810514 / 1

Experiment ID: $18120 S 40$
Test Date: Dec 03, 2018

Report Date: Dec 20, 2018

\section{Panel/Cell Line}

Leukemia

CCRF-CEM

HL-60(TB)

$\mathrm{K}-562$

MOLT-4

RPMI-8226

SR

Non-Small Cell Lung Cancer

A549/ATCC

EKVX

HOP-62

HOP-92

$\mathrm{NCl}-\mathrm{H} 226$

$\mathrm{NCl}-\mathrm{H} 23$

$\mathrm{NCl}-\mathrm{H} 322 \mathrm{M}$

$\mathrm{NCl}-\mathrm{H} 460$

$\mathrm{NCl}-\mathrm{H} 522$

Colon Cancer

COLO 205

HCC-2998

HCT-116

HCT-15

HT29

KM12

SW-620

CNS Cancer

SF-268

SF-295

SF-539

SNB-19

SNB-75

U251

Melanoma

LOX IMVI

MALME-3M

M14

MDA-MB-435

SK-MEL-2

SK-MEL-28

SK-MEL-5

UACC-257

UACC-62

Ovarian Cancer

IGROV1

OVCAR-3

OVCAR-4

OVCAR-5

OVCAR-8

NCI/ADR-RES

SK-OV-3

Renal Cancer

786-0

A498

ACHN

CAKI-1

RXF 393

SN12C

TK-10

UO-31

Prostate Cancer

PC-3

DU-145

Breast Cancer

MCF7

MDA-MB-231/ATCC

HS 578T

BT-549

T-47D

MDA-MB-468

\section{Growth Percent}

94.31

100.54

102.01

95.31

99.82

91.30

99.16

100.85

93.51

75.58

90.93

87.84

84.01

95.97

95.22

94.95

96.70

89.47

94.67

101.89

91.22

95.19

94.23

96.43

92.73

90.48

87.79

95.92

98.18

86.33

94.25

98.86

112.02

99.91

96.73

83.14

90.23

94.16

96.37

104.66

88.82

100.83

93.50

111.74

95.87

89.58

97.86

92.51

106.13

92.10

101.04

81.85

88.08

95.80

92.22

81.25

88.62

83.98

94.17

91.55

94.17

18.59

36.44
Mean Growth Percent - Growth Percent

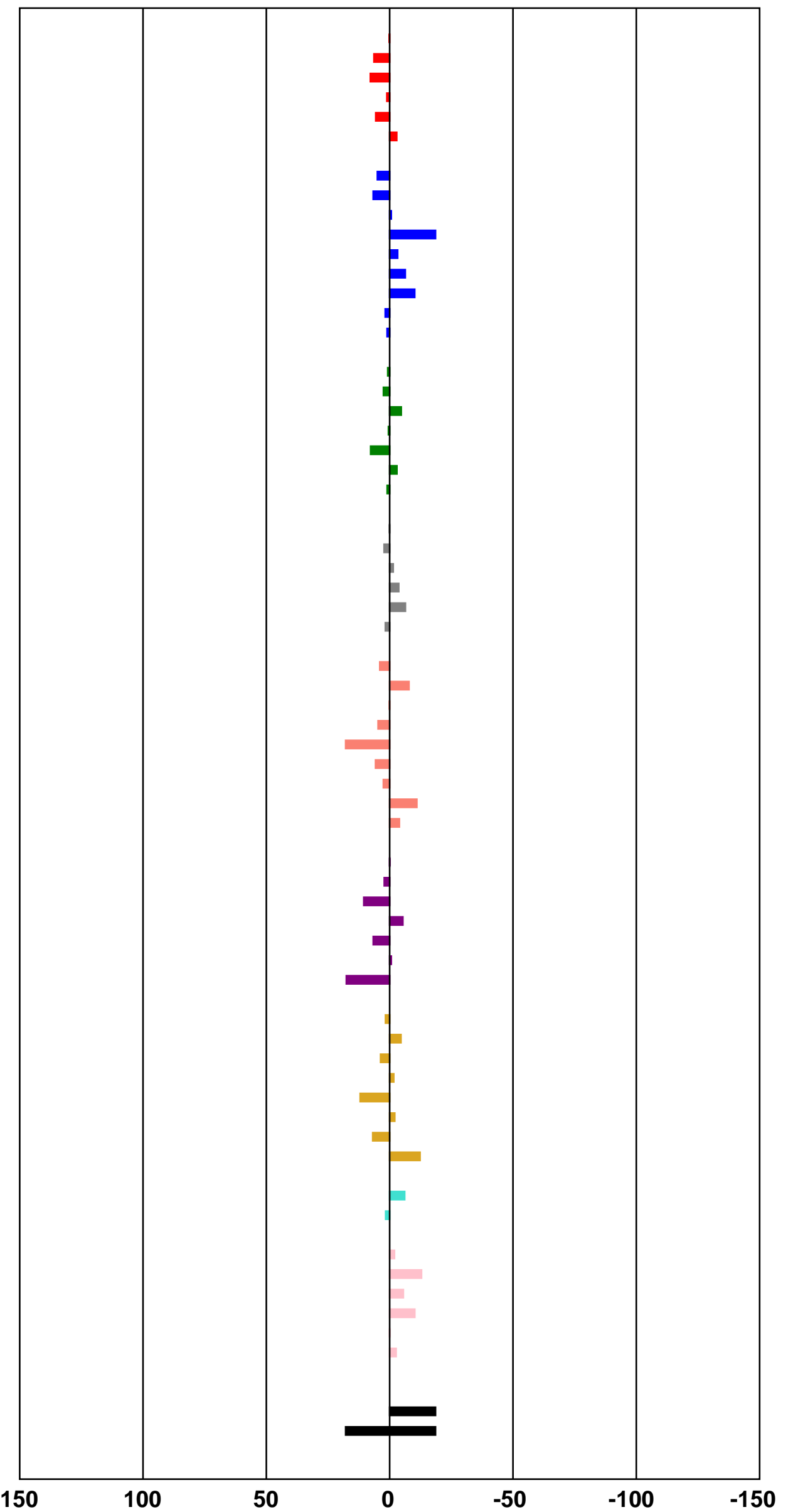


Developmental Therapeutics Program One Dose Mean Graph
NSC: D-810513/1

Experiment ID: $18120 S 40$
Test Date: Dec 03, 2018

Report Date: Dec 20, 2018

\section{Panel/Cell Line}

Leukemia

CCRF-CEM

$\mathrm{K}-562$

MOLT-4

RPMI-8226

SR

Non-Small Cell Lung Cancer

A549/ATCC

EKVX

HOP-62

HOP-92

$\mathrm{NCl}-\mathrm{H} 226$

$\mathrm{NCl}-\mathrm{H} 23$

$\mathrm{NCl}-\mathrm{H} 322 \mathrm{M}$

$\mathrm{NCl}-\mathrm{H} 460$

$\mathrm{NCl}-\mathrm{H} 522$

Colon Cancer

COLO 205

HCC-2998

HCT-116

HCT-15

HT29

KM12

SW-620

CNS Cancer

SF-268

SF-539

SNB-19

SNB-75

U251

Melanoma

LOX IMVI

MALME-3M

M14

MDA-MB-435

SK-MEL-2

SK-MEL-28

SK-MEL-5

UACC-257

UACC-62

Ovarian Cancer

IGROV1

OVCAR-3

OVCAR-4

OVCAR-5

OVCAR-8

$\mathrm{NCI} / \mathrm{ADR}-\mathrm{RES}$

SK-OV-3

Renal Cancer

786-0

A498

ACHN

CAKI-1

RXF 393

SN12C

TK-10

UO-31

Prostate Cancer

PC-3

DU-145

Breast Cancer

MCF7

MDA-MB-231/ATCC

HS 578T

BT-549

T-47D

MDA-MB-468

\section{Growth Percent}

11.43

$-36.72$

9.33

14.23

15.03

9.93

40.43

47.88

28.84

41.03

61.38

34.53

44.01

9.01

11.36

9.01

40.34

21.16

27.89

15.37

20.89

17.36

43.52

33.63

$-14.58$

30.02

$-11.88$

24.43

50.48

46.35

21.97

$-3.04$

39.81

61.40

15.15

56.13

17.26

32.97

$-17.33$

63.70

43.93

29.19

4.42

30.23

41.12

0.16

35.43

31.55

$-10.14$

36.36

54.21

41.46

24.18

12.60

18.74

26.95

4.30

51.80

22.62

$-27.69$

24.25

60.97

100.42
Mean Growth Percent - Growth Percent

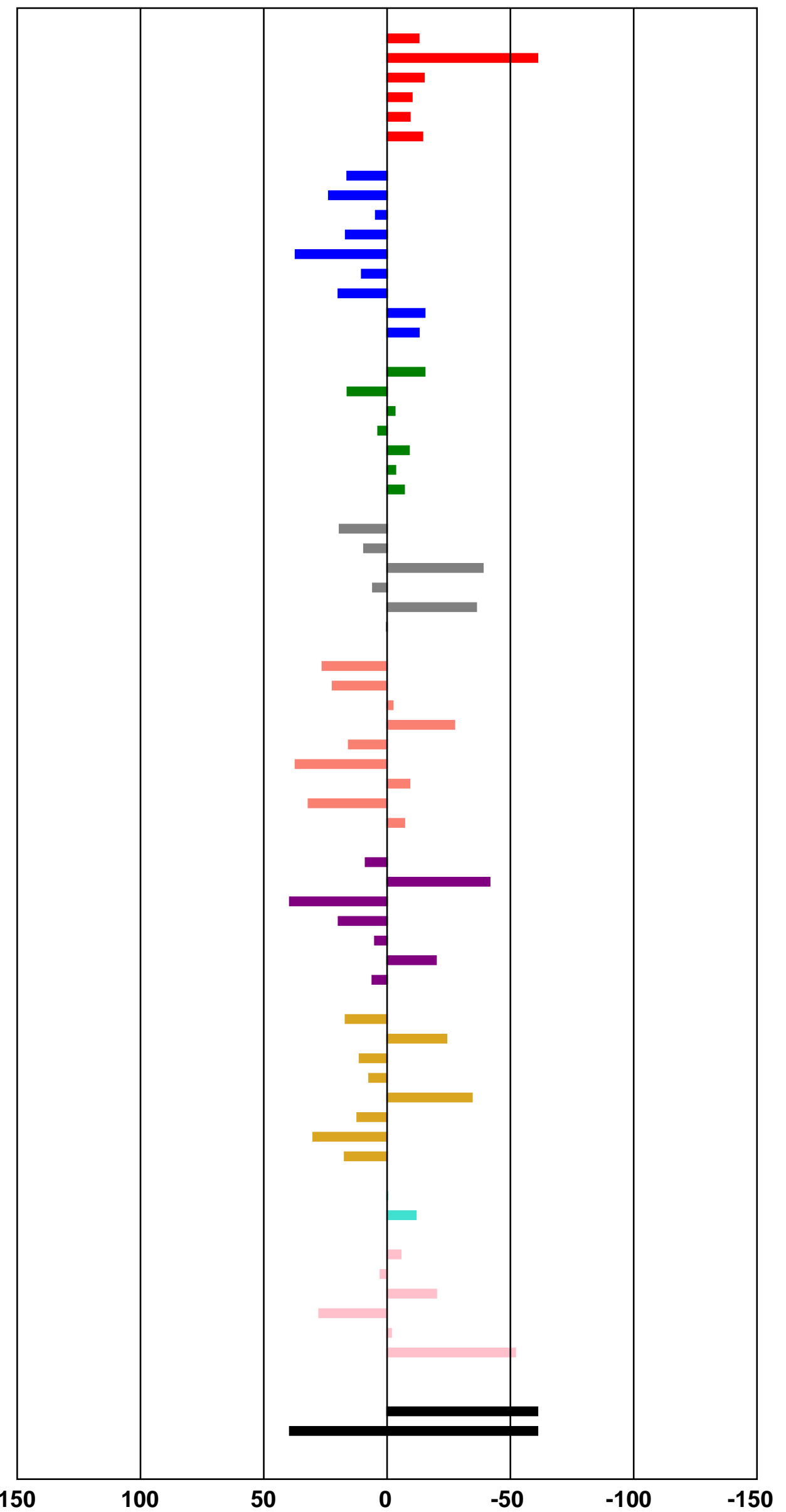


Developmental Therapeutics Program One Dose Mean Graph
NSC: D-810512 / 1

Experiment ID: $18120 S 40$
Test Date: Dec 03, 2018

Report Date: Dec 20, 2018

\section{Panel/Cell Line}

Leukemia

CCRF-CEM

HL-60(TB)

$\mathrm{K}-562$

MOLT-4

RPMI-8226

SR

Non-Small Cell Lung Cancer

A549/ATCC

EKVX

HOP-62

HOP-92

$\mathrm{NCl}-\mathrm{H} 226$

$\mathrm{NCl}-\mathrm{H} 23$

$\mathrm{NCl}-\mathrm{H} 322 \mathrm{M}$

$\mathrm{NCl}-\mathrm{H} 460$

$\mathrm{NCl}-\mathrm{H} 522$

Colon Cancer

COLO 205

HCC-2998

HCT-116

HCT-15

HT29

KM12

SW-620

CNS Cancer

SF-268

SF-539

SNB-19

SNB-75

U251

Melanoma

LOX IMVI

MALME-3M

M14

MDA-MB-435

SK-MEL-2

SK-MEL-28

SK-MEL-5

UACC-257

UACC-62

Ovarian Cancer

IGROV1

OVCAR-3

OVCAR-4

OVCAR-5

OVCAR-8

$\mathrm{NCI} / \mathrm{ADR}-\mathrm{RES}$

SK-OV-3

Renal Cancer

786-0

A498

ACHN

CAKI-1

RXF 393

SN12C

TK-10

UO-31

Prostate Cancer

PC-3

DU-145

Breast Cancer

MCF7

MDA-MB-231/ATCC

HS 578T

BT-549

T-47D

MDA-MB-468

\section{Growth Percent}

86.02

98.35

93.60

82.54

88.80

89.32

93.50

105.65

90.72

76.79

87.96

90.47

88.71

97.03

88.27

94.48

96.67

97.29

97.71

103.51

86.60

89.08

88.04

101.82

86.18

90.64

84.94

91.12

95.85

94.62

99.84

99.63

104.48

97.77

102.18

91.75

87.17

92.76

91.12

101.71

92.92

90.27

92.30

100.76

94.47

78.36

95.81

88.60

92.42

91.44

103.54

82.15

87.27

96.19

92.75

85.72

84.73

90.85

82.63

85.20

92.22

15.43

28.86
Mean Growth Percent - Growth Percent

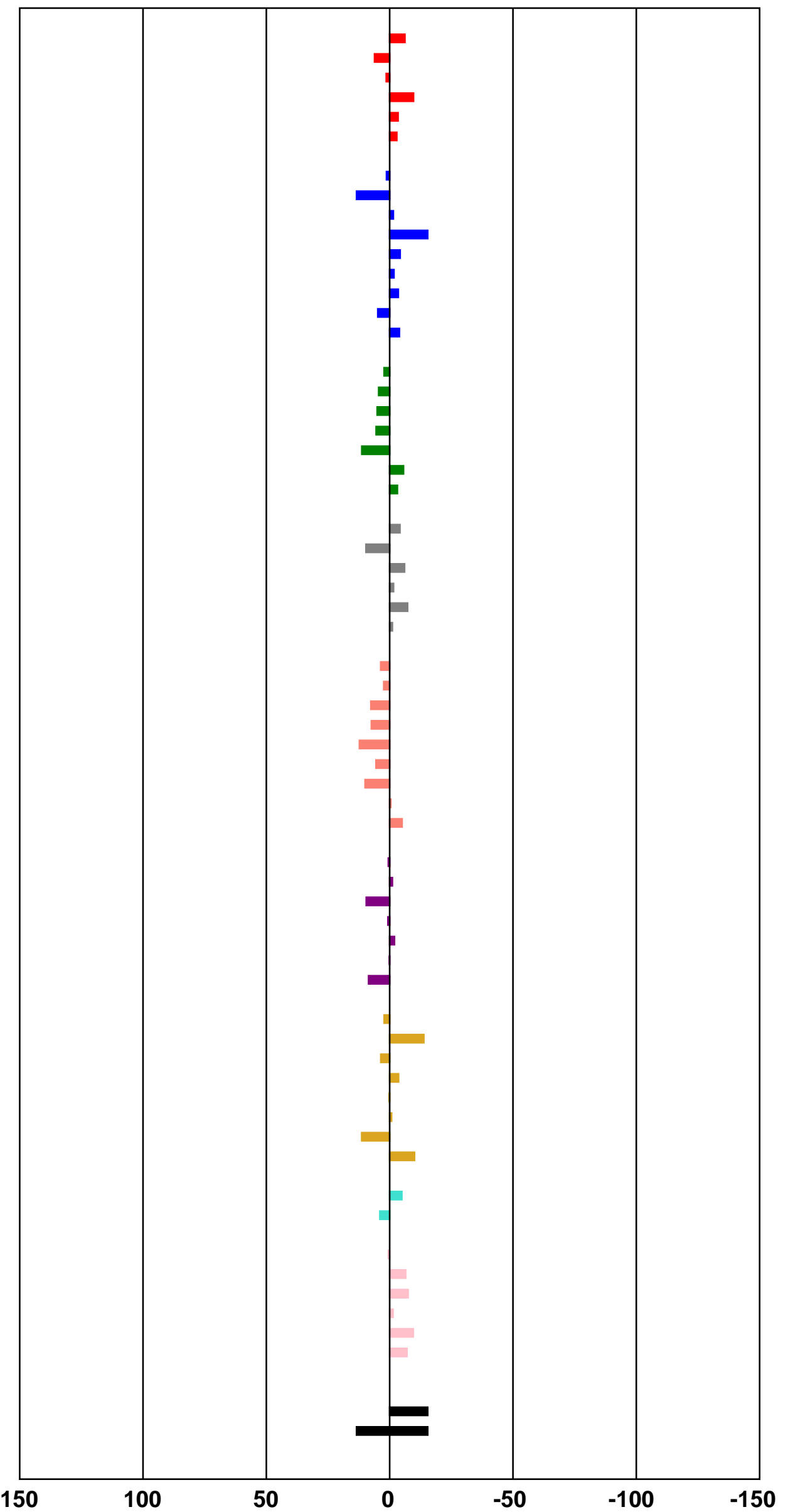


NSC: D-810520/1

\section{Panel/Cell Line}

Leukemia

CCRF-CEM

HL-60(TB)

$\mathrm{K}-562$

MOLT-4

RPMI-8226

$\mathrm{SR}$

Non-Small Cell Lung Cancer

A549/ATCC

EKVX

HOP-62

HOP-92

$\mathrm{NCl}-\mathrm{H} 226$

$\mathrm{NCl}-\mathrm{H} 23$

$\mathrm{NCl}-\mathrm{H} 322 \mathrm{M}$

$\mathrm{NCl}-\mathrm{H} 460$

$\mathrm{NCl}-\mathrm{H} 522$

Colon Cancer

COLO 205

HCC-2998

HCT-116

HCT-15

HT29

KM12

SW-620

CNS Cancer

SF-268

SF-539

SNB-19

SNB-75

U251

Melanoma

LOX IMVI

MALME-3M

M14

MDA-MB-435

SK-MEL-2

SK-MEL-28

SK-MEL-5

UACC-257

UACC-62

Ovarian Cancer

IGROV1

OVCAR-3

OVCAR-4

OVCAR-5

OVCAR-8

NCI/ADR-RES

SK-OV-3

Renal Cancer

786-0

A498

ACHN

CAKI-1

RXF 393

SN12C

TK-10

UO-31

Prostate Cancer

PC-3

DU-145

Breast Cancer

MCF7

MDA-MB-231/ATCC

HS $578 \mathrm{~T}$

BT-549

T-47D

MDA-MB-468

\section{Growth Percent}

9.87

$-38.59$

5.12

5.21

8.83

3.51

8.92

31.47

12.25

$-18.47$

46.67

20.42

44.38

7.09

$-1.80$

2.71

25.11

10.13

22.45

5.34

8.14

22.39

13.73

22.18

$-35.75$

18.35

$-2.82$

4.81

32.90

28.75

10.90

2.82

5.31

19.44

18.57

$-0.63$

$-10.21$

24.34

9.38

25.71

19.19

5.21

2.96

0.75

25.93

$-8.08$

34.60

35.56

8.55

10.69

3.01

35.51

4.35

15.54

15.37

15.61

$-1.41$

23.69

12.85

$-7.54$

11.35

49.94

85.26
Mean Growth Percent - Growth Percent

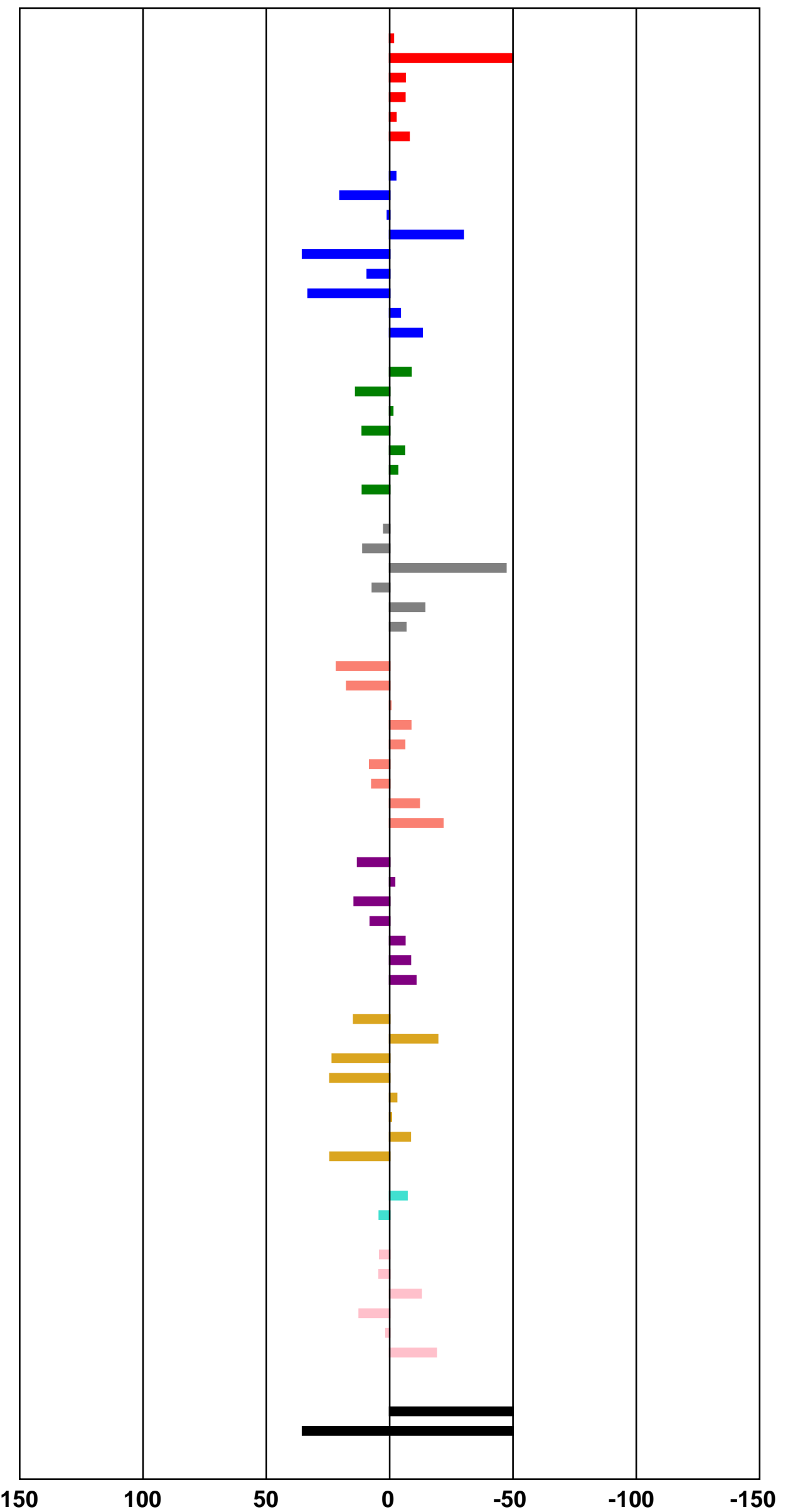


Developmental Therapeutics Program One Dose Mean Graph
NSC: D-810517 / 1

Experiment ID: $18120 S 40$
Test Date: Dec 03, 2018

Report Date: Dec 20, 2018

\section{Panel/Cell Line}

Leukemia

CCRF-CEM

HL-60(TB)

$\mathrm{K}-562$

MOLT-4

RPMI-8226

SR

Non-Small Cell Lung Cancer

A549/ATCC

EKVX

HOP-62

HOP-92

$\mathrm{NCl}-\mathrm{H} 226$

$\mathrm{NCl}-\mathrm{H} 23$

$\mathrm{NCl}-\mathrm{H} 322 \mathrm{M}$

$\mathrm{NCl}-\mathrm{H} 460$

$\mathrm{NCl}-\mathrm{H} 522$

Colon Cancer

COLO 205

HCC-2998

HCT-116

HCT-15

HT29

KM12

SW-620

CNS Cancer

SF-268

SF-539

SNB-19

SNB-75

U251

Melanoma

LOX IMVI

MALME-3M

M14

MDA-MB-435

SK-MEL-2

SK-MEL-28

SK-MEL-5

UACC-257

UACC-62

Ovarian Cancer

IGROV1

OVCAR-3

OVCAR-4

OVCAR-5

OVCAR-8

$\mathrm{NCI} / \mathrm{ADR}-\mathrm{RES}$

SK-OV-3

Renal Cancer

786-0

A498

ACHN

CAKI-1

RXF 393

SN12C

TK-10

UO-31

Prostate Cancer

PC-3

DU-145

Breast Cancer

MCF7

MDA-MB-231/ATCC

HS 578T

BT-549

T-47D

MDA-MB-468

\section{Growth Percent}

10.66

$-27.98$

10.42

20.24

11.99

16.46

37.59

41.88

31.61

54.17

62.14

41.14

48.69

15.18

$-0.25$

1.46

64.29

25.41

31.51

14.84

24.75

24.30

53.51

34.16

6.39

35.83

$-1.76$

23.61

56.89

50.28

19.37

$-30.19$

40.19

64.88

28.23

44.54

32.14

39.09

1.73

70.41

47.99

24.61

9.69

32.69

42.67

3.93

36.25

28.91

2.78

44.93

61.90

42.47

23.45

18.04

22.07

52.43

13.44

62.57

29.78

6.05

28.94

59.13

100.60
Mean Growth Percent - Growth Percent

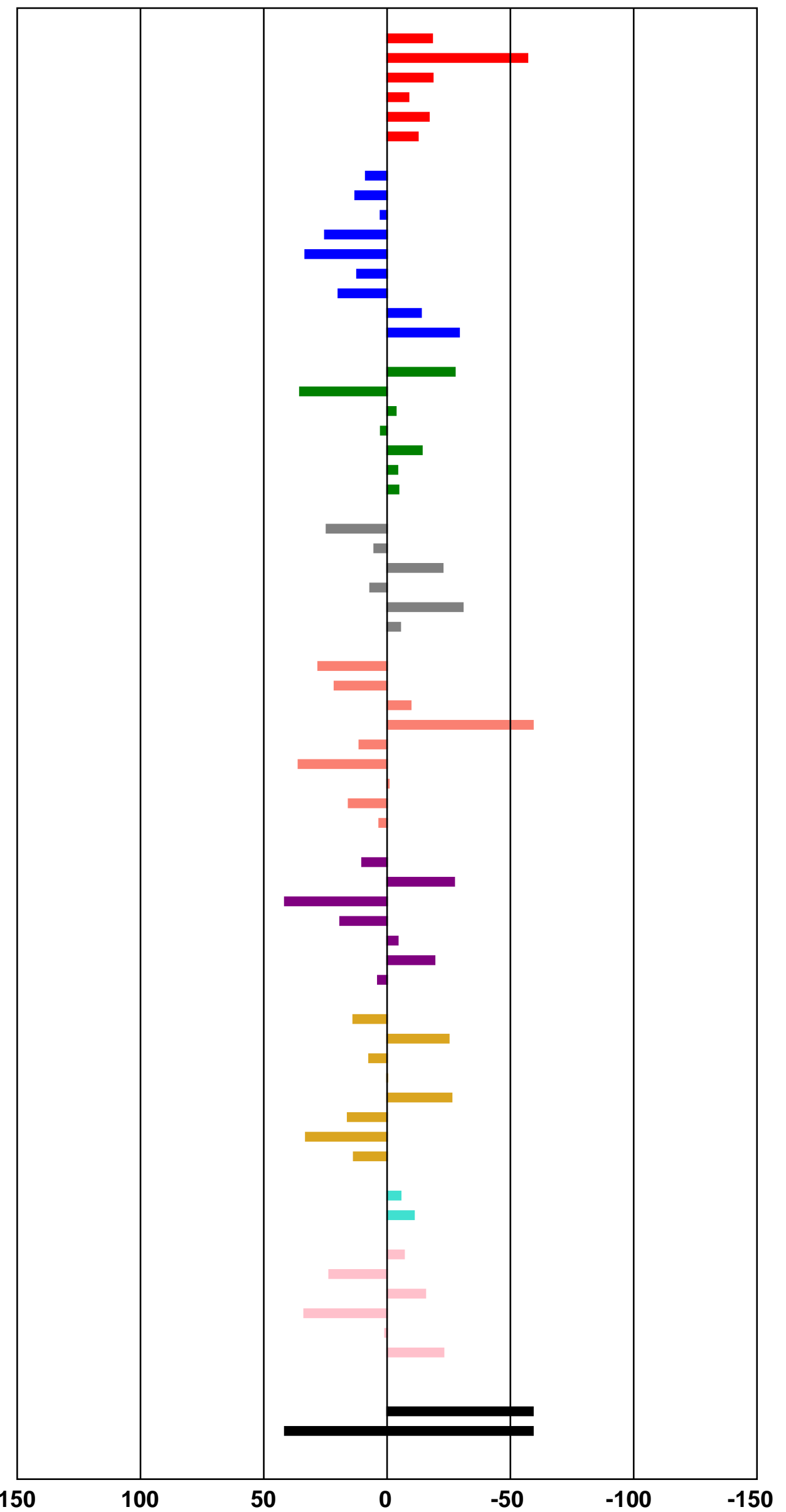


Developmental Therapeutics Program One Dose Mean Graph
NSC: D-810511 / 1

Experiment ID: $18120 S 40$
Test Date: Dec 03, 2018

Report Date: Dec 20, 2018

\section{Panel/Cell Line}

Leukemia

CCRF-CEM

$\mathrm{K}-562$

MOLT-4

RPMI-8226

SR

Non-Small Cell Lung Cancer

A549/ATCC

EKVX

HOP-62

HOP-92

$\mathrm{NCl}-\mathrm{H} 226$

$\mathrm{NCl}-\mathrm{H} 23$

$\mathrm{NCl}-\mathrm{H} 322 \mathrm{M}$

$\mathrm{NCl}-\mathrm{H} 460$

$\mathrm{NCl}-\mathrm{H} 522$

Colon Cancer

COLO 205

HCC-2998

HCT-116

HCT-15

HT29

KM12

SW-620

CNS Cancer

SF-268

SF-539

SNB-19

SNB-75

U251

Melanoma

LOX IMVI

MALME-3M

M14

MDA-MB-435

SK-MEL-2

SK-MEL-28

SK-MEL-5

UACC-257

UACC-62

Ovarian Cancer

IGROV1

OVCAR-3

OVCAR-4

OVCAR-5

OVCAR-8

$\mathrm{NCI} / \mathrm{ADR}-\mathrm{RES}$

SK-OV-3

Renal Cancer

786-0

A498

ACHN

CAKI-1

RXF 393

SN12C

TK-10

UO-31

Prostate Cancer

PC-3

DU-145

Breast Cancer

MCF7

MDA-MB-231/ATCC

HS 578T

BT-549

T-47D

MDA-MB-468

\section{Growth Percent}

7.70

$-35.97$

11.32

15.82

13.91

12.69

40.09

52.95

27.09

43.00

67.25

40.84

61.86

12.78

11.03

23.90

56.22

18.72

20.80

13.85

24.60

11.12

39.94

28.94

3.63

35.33

$-6.53$

24.95

45.93

44.35

18.09

$-9.00$

33.60

53.52

19.19

66.07

23.64

36.12

$-7.12$

67.83

51.00

41.46

$-2.19$

35.48

37.77

$-6.87$

30.83

40.62

10.37

33.82

64.10

42.28

29.16

38.06

16.12

35.18

$-8.02$

47.14

18.62

$-12.44$

26.88

62.85

103.80
Mean Growth Percent - Growth Percent

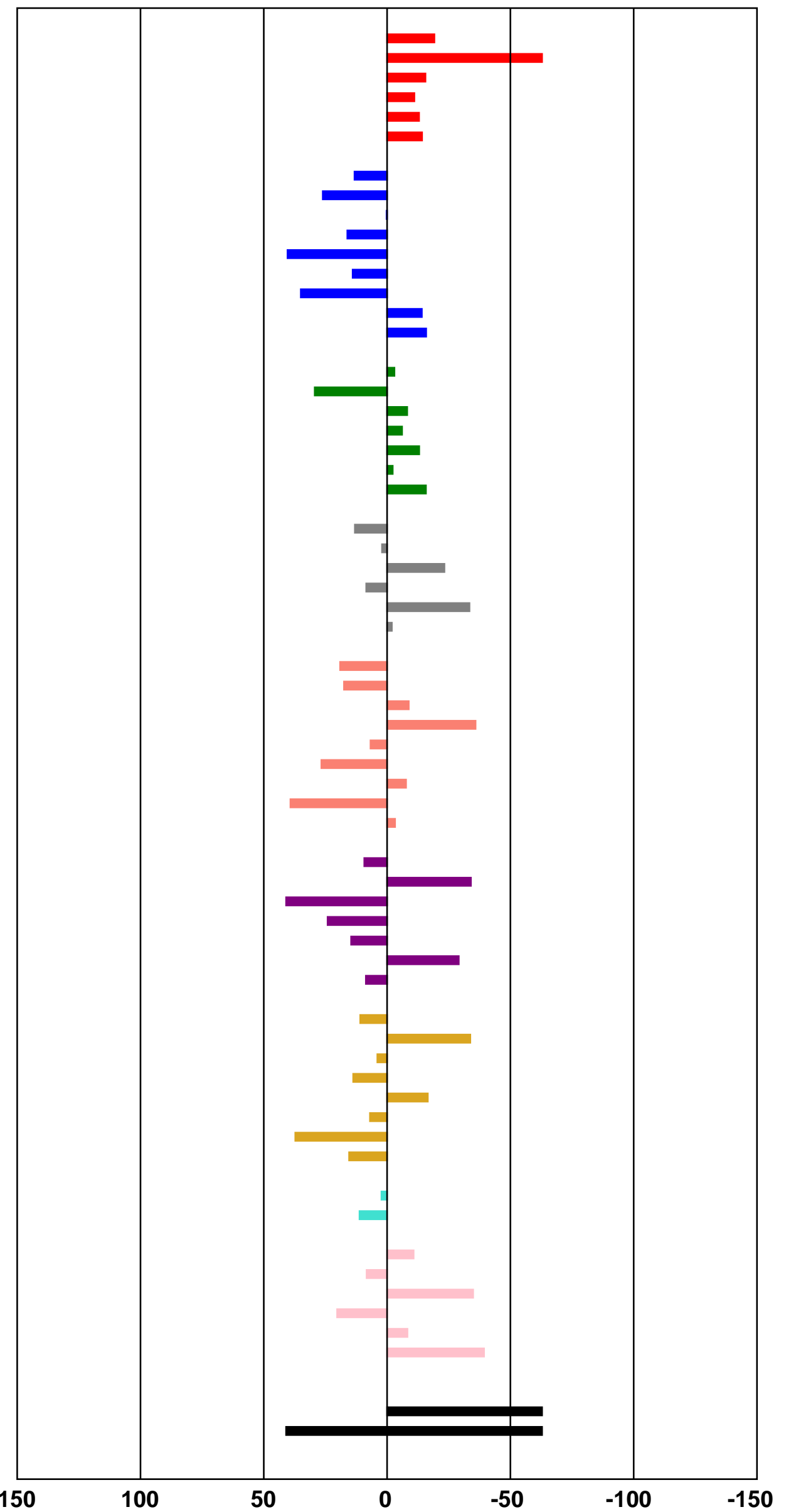


Developmental Therapeutics Program One Dose Mean Graph
NSC: D-810518/1

Experiment ID: $18120 S 40$
Test Date: Dec 03, 2018

Report Date: Dec 20, 2018

\section{Panel/Cell Line}

Leukemia

CCRF-CEM

HL-60(TB)

$\mathrm{K}-562$

MOLT-4

RPMI-8226

SR

Non-Small Cell Lung Cancer

A549/ATCC

EKVX

HOP-62

HOP-92

$\mathrm{NCl}-\mathrm{H} 226$

$\mathrm{NCl}-\mathrm{H} 23$

$\mathrm{NCl}-\mathrm{H} 322 \mathrm{M}$

$\mathrm{NCl}-\mathrm{H} 460$

$\mathrm{NCl}-\mathrm{H} 522$

Colon Cancer

COLO 205

HCC-2998

HCT-116

HCT-15

HT29

KM12

SW-620

CNS Cancer

SF-268

SF-539

SNB-19

SNB-75

U251

Melanoma

LOX IMVI

MALME-3M

M14

MDA-MB-435

SK-MEL-2

SK-MEL-28

SK-MEL-5

UACC-257

UACC-62

Ovarian Cancer

IGROV1

OVCAR-3

OVCAR-4

OVCAR-5

OVCAR-8

$\mathrm{NCI} / \mathrm{ADR}-\mathrm{RES}$

SK-OV-3

Renal Cancer

786-0

A498

ACHN

CAKI-1

RXF 393

SN12C

TK-10

UO-31

Prostate Cancer

PC-3

DU-145

Breast Cancer

MCF7

MDA-MB-231/ATCC

HS 578T

BT-549

T-47D

MDA-MB-468

\section{Growth Percent}

75.39

99.85

89.79

94.11

86.48

80.27

94.27

94.35

93.85

88.46

81.66

89.22

100.68

103.29

85.37

94.42

105.23

91.65

94.24

99.40

103.62

99.51

93.17

96.59

97.69

96.68

96.80

91.92

95.74

93.08

96.90

100.73

106.23

104.90

96.74

95.08

84.62

96.87

102.80

86.46

105.06

94.71

94.10

104.67

93.58

102.49

101.60

87.51

112.38

93.69

99.07

82.31

86.22

99.86

85.07

88.52

94.69

91.83

78.44

74.24

94.14

19.90

38.14
Mean Growth Percent - Growth Percent

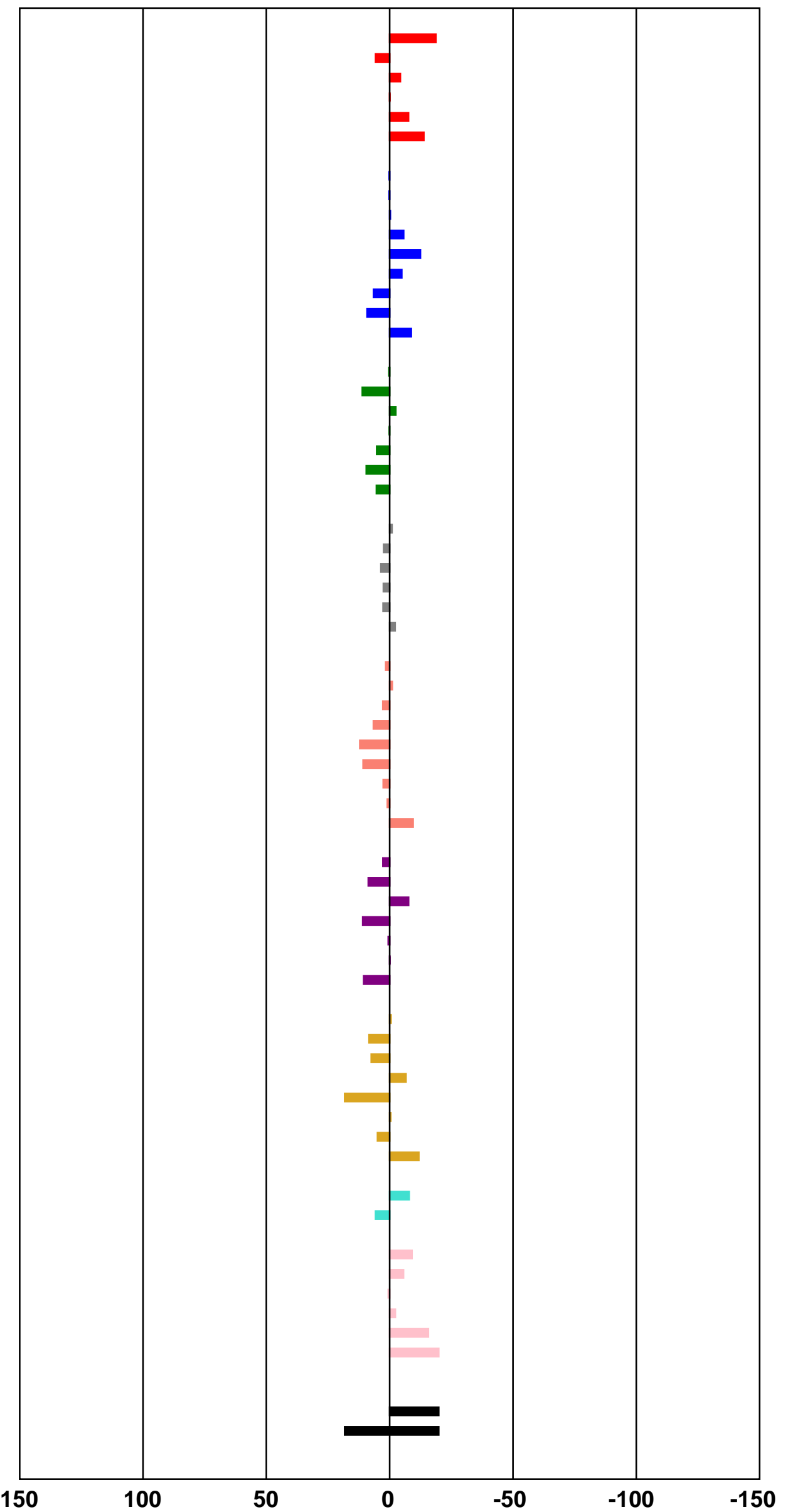


10 One Dose Mean Graph

\begin{tabular}{l|l} 
NSC: D-816478/1 Conc: $1.00 E-5$ Molar
\end{tabular}

Experiment ID: $19060 S 28$
Test Date: Jun 17, 2019

Report Date: Jul 26, 2019

\section{Panel/Cell Line}

Leukemia

CCRF-CEM

HL-60(TB)

$\mathrm{K}-562$

MOLT-4

RPMI-8226

$\mathrm{SR}$

Non-Small Cell Lung Cancer

A549/ATCC

HOP-62

HOP-92

$\mathrm{NCl}-\mathrm{H} 23$

$\mathrm{NCl}-\mathrm{H} 322 \mathrm{M}$

$\mathrm{NCl}-\mathrm{H} 460$

$\mathrm{NCl}-\mathrm{H} 522$

Colon Cancer

HCC-2998

HCT-116

HCT-15

HT29

KM12

SW-620

CNS Cancer

SF-268

SF-295

SF-539

SNB-19

SNB-75

U251

Melanoma

LOX IMVI

MALME-3M

M14

MDA-MB-435

SK-MEL-2

SK-MEL-28

SK-MEL-5

UACC-257

UACC-62

Ovarian Cancer

IGROV1

OVCAR-3

OVCAR-4

OVCAR-5

OVCAR-8

NCI/ADR-RES

Renal Cancer

786-0

ACHN

CAKI-1

RXF 393

SN12C

TK-10

UO-31

Prostate Cancer

PC-3

DU-145

Breast Cancer

MCF7

MDA-MB-231/ATCC

HS 578T

BT-549

MDA-MB-468

\section{Growth Percent}

35.52

24.29

14.57

31.96

37.64

33.78

40.17

36.42

57.02

58.85

69.02

7.82

9.20

69.15

11.79

17.52

12.02

20.50

12.37

50.07

19.88

26.69

37.59

18.77

39.91

39.17

47.92

9.71

$-3.14$

23.86

53.85

14.98

55.13

27.29

39.81

$-2.00$

59.03

48.82

49.32

8.09

50.79

46.61

38.84

26.51

42.23

64.92

55.34

49.72

49.73

15.59

44.65

$-2.93$

40.43

$-0.81$

33.07

36.21

72.29
Mean Growth Percent - Growth Percent

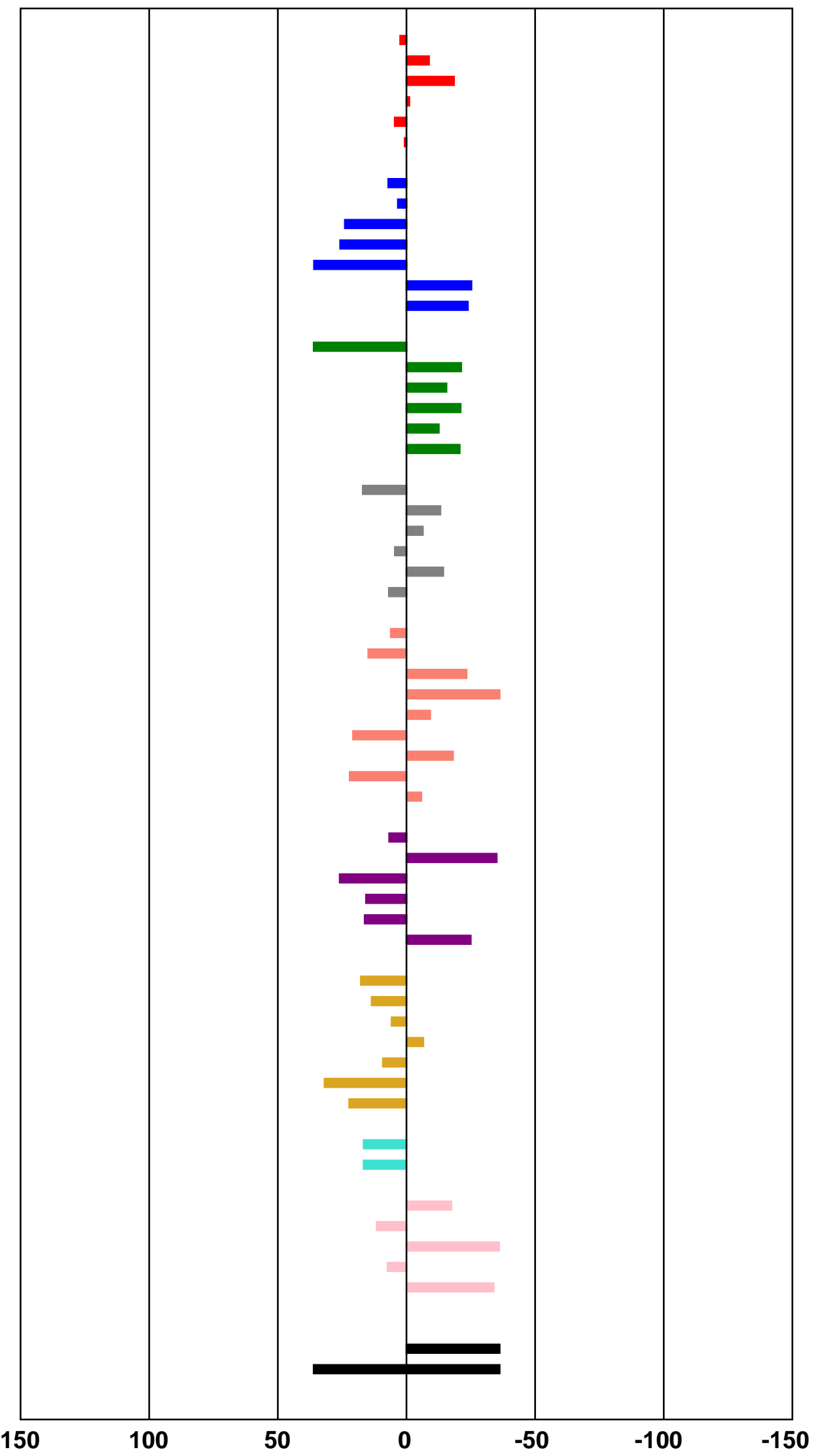


10 One Dose Mean Graph

\section{Panel/Cell Line}

Leukemia

CCRF-CEM

HL-60(TB)

$\mathrm{K}-562$

MOLT-4

RPMI-8226

$\mathrm{SR}$

Non-Small Cell Lung Cancer

A549/ATCC

EKVX

HOP-62

HOP-92

$\mathrm{NCl}-\mathrm{H} 23$

$\mathrm{NCl}-\mathrm{H} 322 \mathrm{M}$

$\mathrm{NCl}-\mathrm{H} 460$

$\mathrm{NCl}-\mathrm{H} 522$

Colon Cancer

HCC-2998

HCT-116

HCT-15

HT29

KM12

SW-620

CNS Cancer

SF-268

SF-295

SF-539

SNB-19

SNB-75

U251

Melanoma

LOX IMVI

MALME-3M

M14

MDA-MB-435

SK-MEL-2

SK-MEL-28

SK-MEL-5

UACC-257

UACC-62

Ovarian Cancer

IGROV1

OVCAR-3

OVCAR-4

OVCAR-5

OVCAR-8

NCI/ADR-RES

Renal Cancer

786-0

ACHN

CAKI-1

RXF 393

SN12C

TK-10

UO-31

Prostate Cancer

PC-3

DU-145

Breast Cancer

MCF7

MDA-MB-231/ATCC

HS 578T

BT-549

MDA-MB-468

Mean

Delta

Range

\section{Growth Percent}

Mean Growth Percent - Growth Percent

76.22

76.31

22.75

48.56

74.93

40.78

54.71

69.95

54.79

63.04

71.87

91.77

33.18

50.99

85.87

38.77

29.66

19.02

40.25

20.08

73.20

49.96

65.15

67.75

44.87

56.47

50.83

58.26

40.92

1.55

46.59

66.31

51.28

72.52

49.39

62.83

27.38

60.79

78.58

81.53

30.30

81.29

80.56

48.27

83.91

81.51

79.82

69.80

67.04

81.21

29.38

69.65

42.42

58.58

12.51

56.11

54.56

90.22

\section{Mean Growth Percent - Growth Percent}

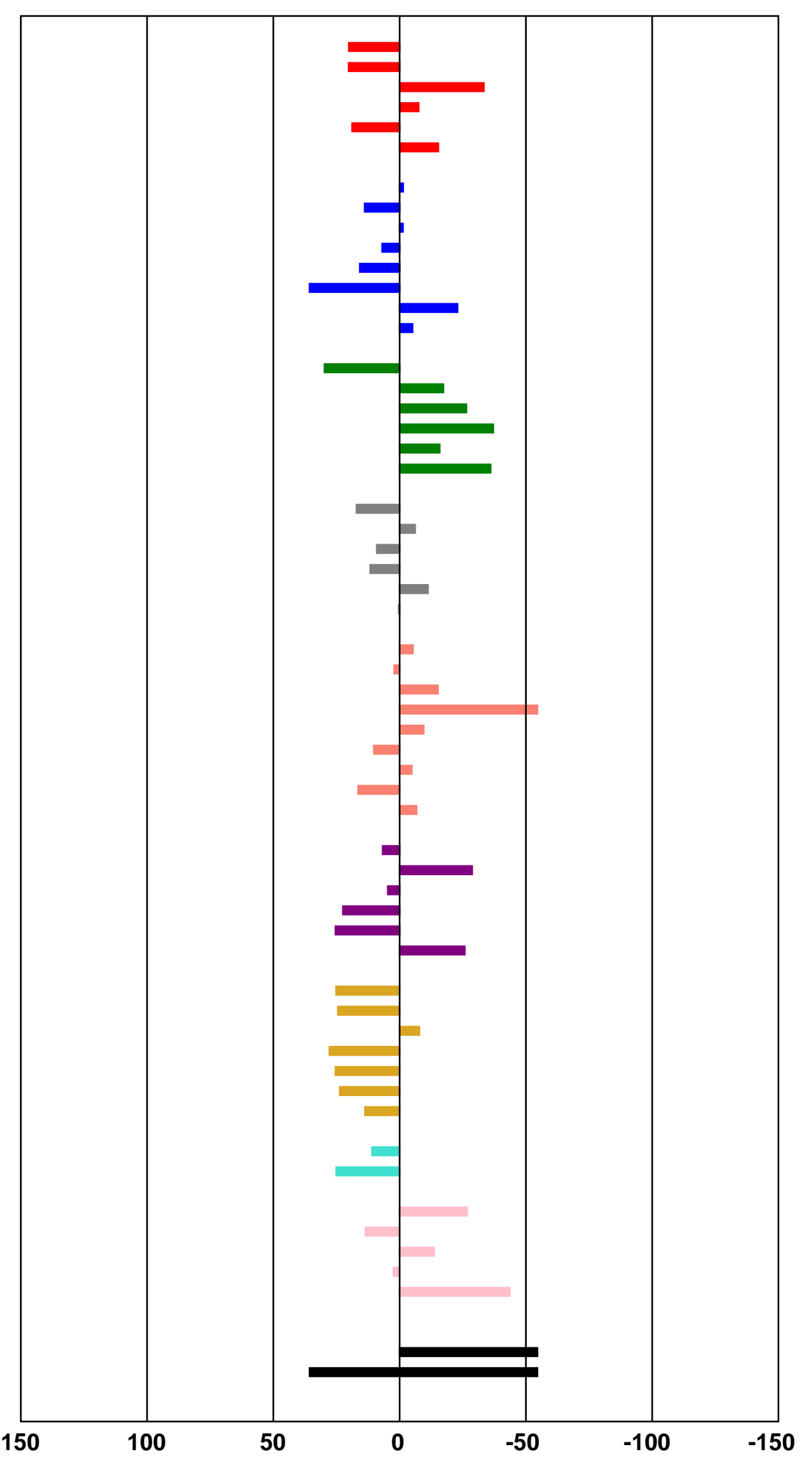




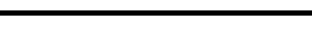
One Dose Mean Graph

\section{Panel/Cell Line}

Leukemia

CCRF-CEM

HL-60(TB)

$\mathrm{K}-562$

MOLT-4

RPMI-8226

$\mathrm{SR}$

Non-Small Cell Lung Cancer

A549/ATCC

EKVX

HOP-62

HOP-92

$\mathrm{NCl}-\mathrm{H} 23$

$\mathrm{NCl}-\mathrm{H} 322 \mathrm{M}$

$\mathrm{NCl}-\mathrm{H} 460$

$\mathrm{NCl}-\mathrm{H} 522$

Colon Cancer

HCC-2998

HCT-116

HCT-15

HT29

KM12

SW-620

CNS Cancer

SF-268

SF-295

SF-539

SNB-19

SNB-75

U251

Melanoma

LOX IMVI

MALME-3M

M14

MDA-MB-435

SK-MEL-2

SK-MEL-28

SK-MEL-5

UACC-257

UACC-62

Ovarian Cancer

IGROV1

OVCAR-3

OVCAR-4

OVCAR-5

OVCAR-8

NCI/ADR-RES

Renal Cancer

786-0

ACHN

CAKI-1

RXF 393

SN12C

TK-10

UO-31

Prostate Cancer

PC-3

DU-145

Breast Cancer

MCF7

MDA-MB-231/ATCC

HS 578T

BT-549

MDA-MB-468

\section{Growth Percent}

18.85

18.65

16.21

32.40

24.56

43.67

25.53

33.82

23.95

47.96

39.62

40.73

4.29

6.78

38.23

26.09

23.91

10.15

20.02

12.83

47.66

14.89

$-13.45$

30.89

$-3.40$

22.12

45.56

53.31

14.12

$-32.80$

37.41

58.28

24.98

63.11

26.81

27.02

$-17.78$

46.35

34.21

33.28

5.86

46.29

45.08

35.89

$-19.42$

41.23

51.31

43.97

14.23

15.33

17.93

28.40

$-18.55$

56.16

7.72

25.31

58.11

95.91
Mean Growth Percent - Growth Percent

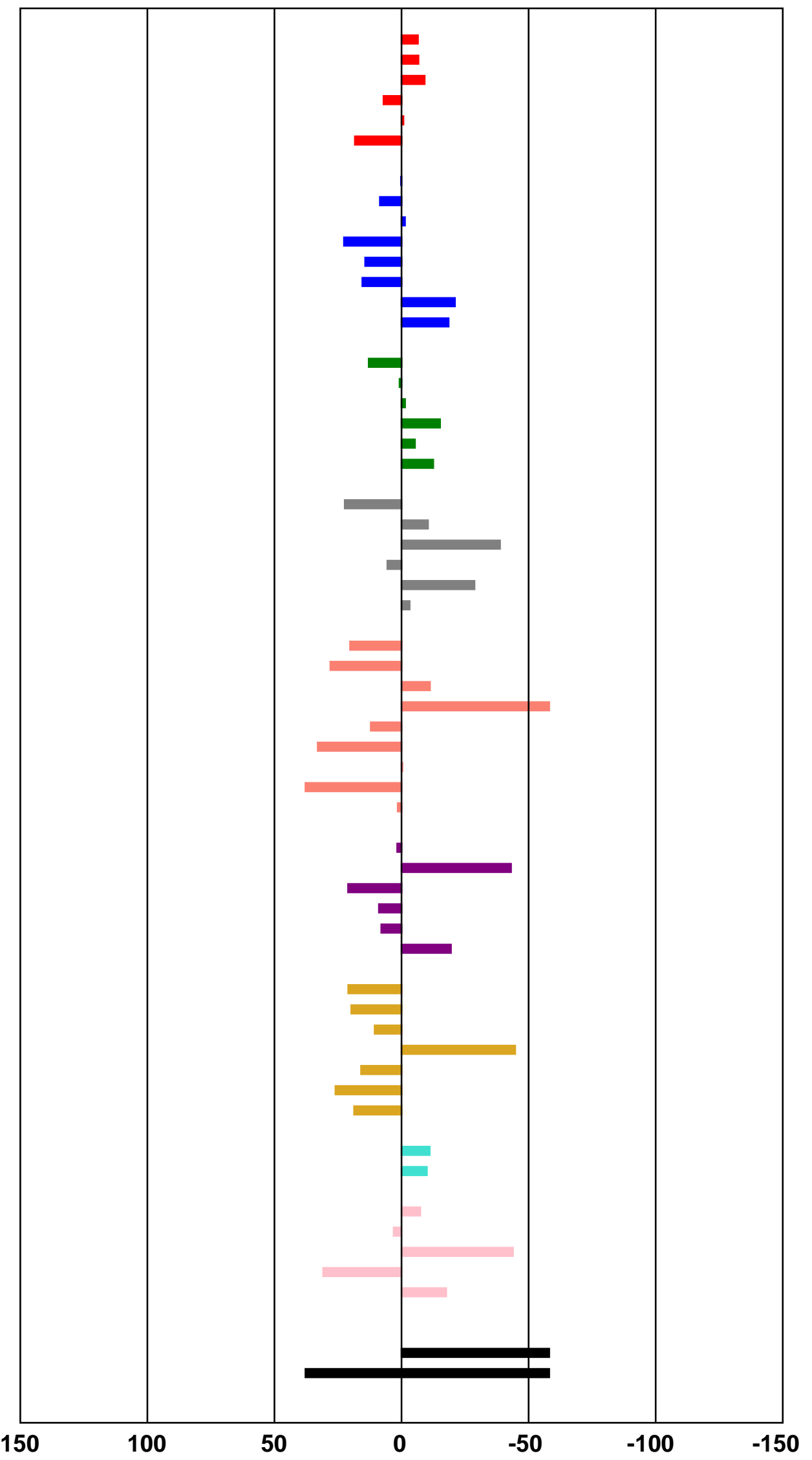


Developmental Therapeutics Program One Dose Mean Graph
NSC: D-816479 / 1

Experiment ID: 19060 S28
Test Date: Jun 17, 2019

Report Date: Jul 26, 2019

\section{Panel/Cell Line}

Leukemia

CCRF-CEM

HL-60(TB)

$\mathrm{K}-562$

MOLT-4

RPMI-8226

SR

Non-Small Cell Lung Cancer

A549/ATCC

HOP-62

HOP-92

$\mathrm{NCl}-\mathrm{H} 23$

$\mathrm{NCl}-\mathrm{H} 322 \mathrm{M}$

$\mathrm{NCl}-\mathrm{H} 460$

$\mathrm{NCl}-\mathrm{H} 522$

Colon Cancer

HCC-2998

HCT-116

HCT-15

HT29

KM12

SW-620

CNS Cancer

SF-268

SF-295

SF-539

SNB-19

SNB-75

U251

Melanoma

LOX IMVI

MALME-3M

M14

MDA-MB-435

SK-MEL-2

SK-MEL-28

SK-MEL-5

UACC-257

UACC-62

Ovarian Cancer

IGROV1

OVCAR-3

OVCAR-4

OVCAR-5

OVCAR-8

NCI/ADR-RES

Renal Cancer

786-0

ACHN

CAKI-1

RXF 393

SN12C

TK-10

UO-31

Prostate Cancer

PC-3

DU-145

Breast Cancer

MCF7

MDA-MB-231/ATCC

HS 578T

BT-549

MDA-MB-468

\section{Growth Percent}

92.19

103.30

108.15

104.98

86.67

109.09

95.51

92.87

68.52

100.94

100.72

99.68

93.95

110.37

90.71

93.50

118.87

99.67

95.86

96.00

91.61

95.72

90.07

93.79

90.88

101.72

95.27

93.68

100.31

112.25

100.28

92.28

91.00

71.43

96.02

106.58

94.93

92.69

90.28

98.13

97.13

98.13

91.64

95.43

91.56

108.78

82.21

89.88

103.51

90.45

82.20

93.98

101.02

79.14

95.66

27.14

50.35
Mean Growth Percent - Growth Percent

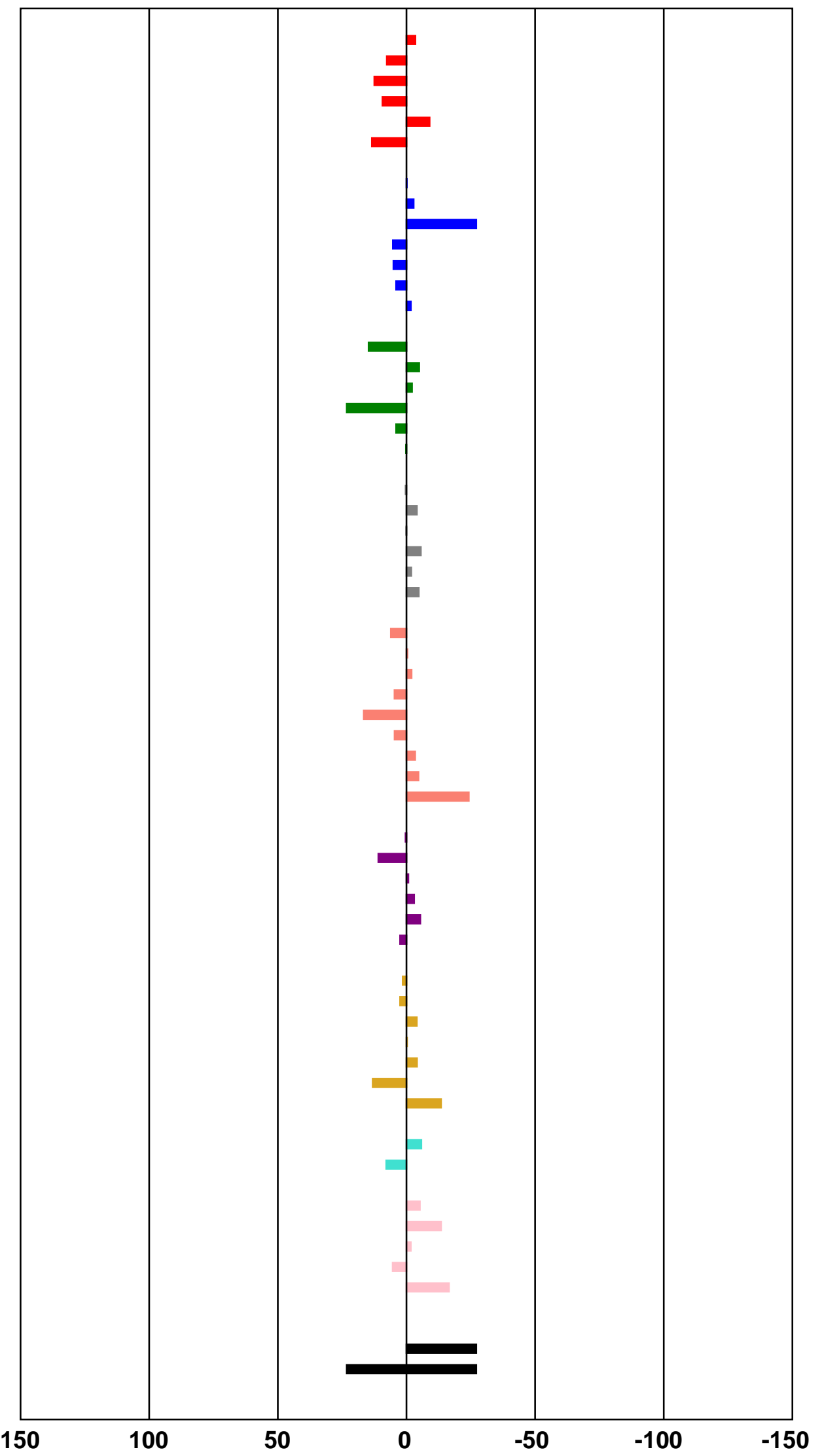


Developmental Therapeutics Program One Dose Mean Graph
NSC: D-823369 / 1

Experiment ID: 20020 S20
Test Date: Feb 24, 2020

Report Date: Mar 24, 2020

\section{Panel/Cell Line}

Leukemia

CCRF-CEM

HL-60(TB)

$\mathrm{K}-562$

MOLT-4

RPMI-8226

Non-Small Cell Lung Cancer

A549/ATCC

EKVX

HOP-62

HOP-92

$\mathrm{NCl}-\mathrm{H} 226$

$\mathrm{NCl}-\mathrm{H} 23$

$\mathrm{NCl}-\mathrm{H} 322 \mathrm{M}$

$\mathrm{NCl}-\mathrm{H} 460$

$\mathrm{NCl}-\mathrm{H} 522$

Colon Cancer

COLO 205

HCC-2998

HCT-116

HCT-15

HT29

KM12

SW-620

CNS Cancer

SF-268

SF-295

SF-539

SNB-19

SNB-75

Melanoma

LOX IMVI

MALME-3M

M14

MDA-MB-435

SK-MEL-2

SK-MEL-28

SK-MEL-5

UACC-257

UACC-62

Ovarian Cancer

IGROV1

OVCAR-3

OVCAR-4

OVCAR-5

OVCAR-8

$\mathrm{NCl} / \mathrm{ADR}$-RES

SK-OV-3

Renal Cancer

786-0

A498

ACHN

CAKI-1

RXF 393

SN12C

TK-10

UO-31

Prostate Cancer

PC-3

DU-145

Breast Cancer

MCF7

MDA-MB-231/ATCC

HS 578T

BT-549

T-47D

MDA-MB-468

\section{Growth Percent}

18.59

$-0.12$

10.91

24.35

21.65

26.50

38.11

31.50

51.22

58.97

28.18

36.48

12.65

$-1.33$

4.78

39.30

24.89

24.31

1.27

33.35

21.02

53.60

1.37

$-13.47$

43.56

5.51

43.26

37.74

10.85

$-5.62$

33.34

55.30

8.59

55.38

35.28

29.16

$-17.77$

52.59

40.23

26.50

0.51

41.63

41.38

$-21.03$

53.58

38.84

$-10.69$

51.42

48.40

53.67

28.06

8.88

15.32

44.19

9.44

47.02

33.86

$-34.17$

25.04

59.21

93.14
Mean Growth Percent - Growth Percent

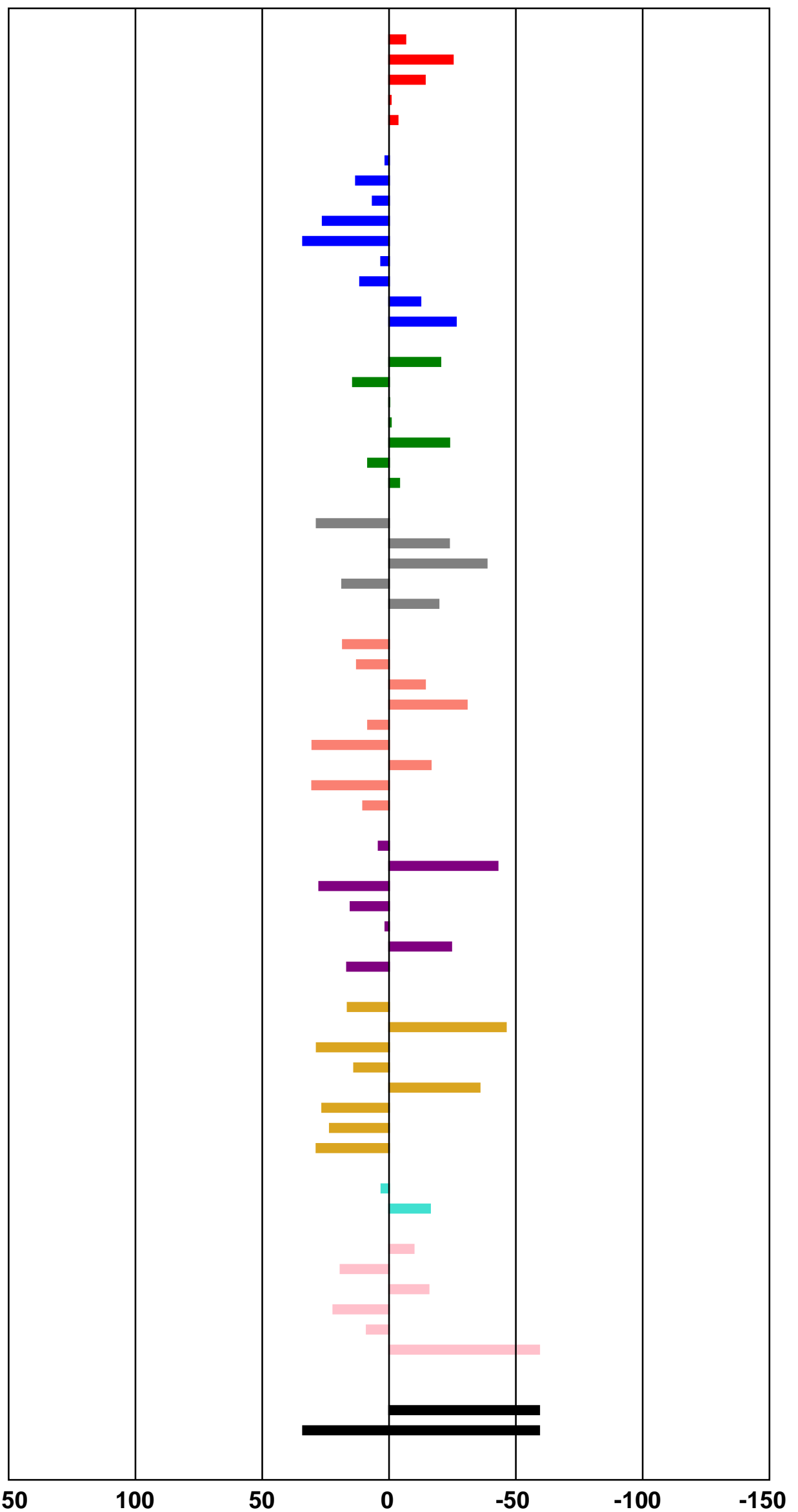


Developmental Therapeutics Program One Dose Mean Graph
NSC: D-816477 / 1

Experiment ID: 19060 S28
Test Date: Jun 17, 2019

Report Date: Jul 26, 2019

\section{Panel/Cell Line}

Leukemia

CCRF-CEM

HL-60(TB)

$\mathrm{K}-562$

MOLT-4

RPMI-8226

SR

Non-Small Cell Lung Cancer

A549/ATCC

HOP-62

HOP-92

$\mathrm{NCl}-\mathrm{H} 23$

$\mathrm{NCl}-\mathrm{H} 322 \mathrm{M}$

$\mathrm{NCl}-\mathrm{H} 460$

$\mathrm{NCl}-\mathrm{H} 522$

Colon Cancer

HCC-2998

HCT-116

HCT-15

HT29

KM12

SW-620

CNS Cancer

SF-268

SF-295

SF-539

SNB-19

SNB-75

U251

Melanoma

LOX IMVI

MALME-3M

M14

MDA-MB-435

SK-MEL-2

SK-MEL-28

SK-MEL-5

UACC-257

UACC-62

Ovarian Cancer

IGROV1

OVCAR-3

OVCAR-4

OVCAR-5

OVCAR-8

NCI/ADR-RES

Renal Cancer

786-0

ACHN

CAKI-1

RXF 393

SN12C

TK-10

UO-31

Prostate Cancer

PC-3

DU-145

Breast Cancer

MCF7

MDA-MB-231/ATCC

HS 578T

BT-549

MDA-MB-468

\section{Growth Percent}

107.08

106.24

95.17

102.59

101.69

93.37

93.28

97.39

83.83

99.68

102.11

97.38

90.93

110.97

100.11

94.96

111.10

95.52

89.74

95.81

92.60

98.54

90.35

82.91

96.75

104.88

90.34

92.78

78.86

102.27

93.74

92.72

92.41

71.16

95.63

106.45

85.30

94.03

91.90

90.33

97.79

97.98

75.04

105.01

95.97

99.12

78.19

88.10

105.91

87.35

92.08

86.63

93.78

89.57

94.54

23.38

39.94
Mean Growth Percent - Growth Percent

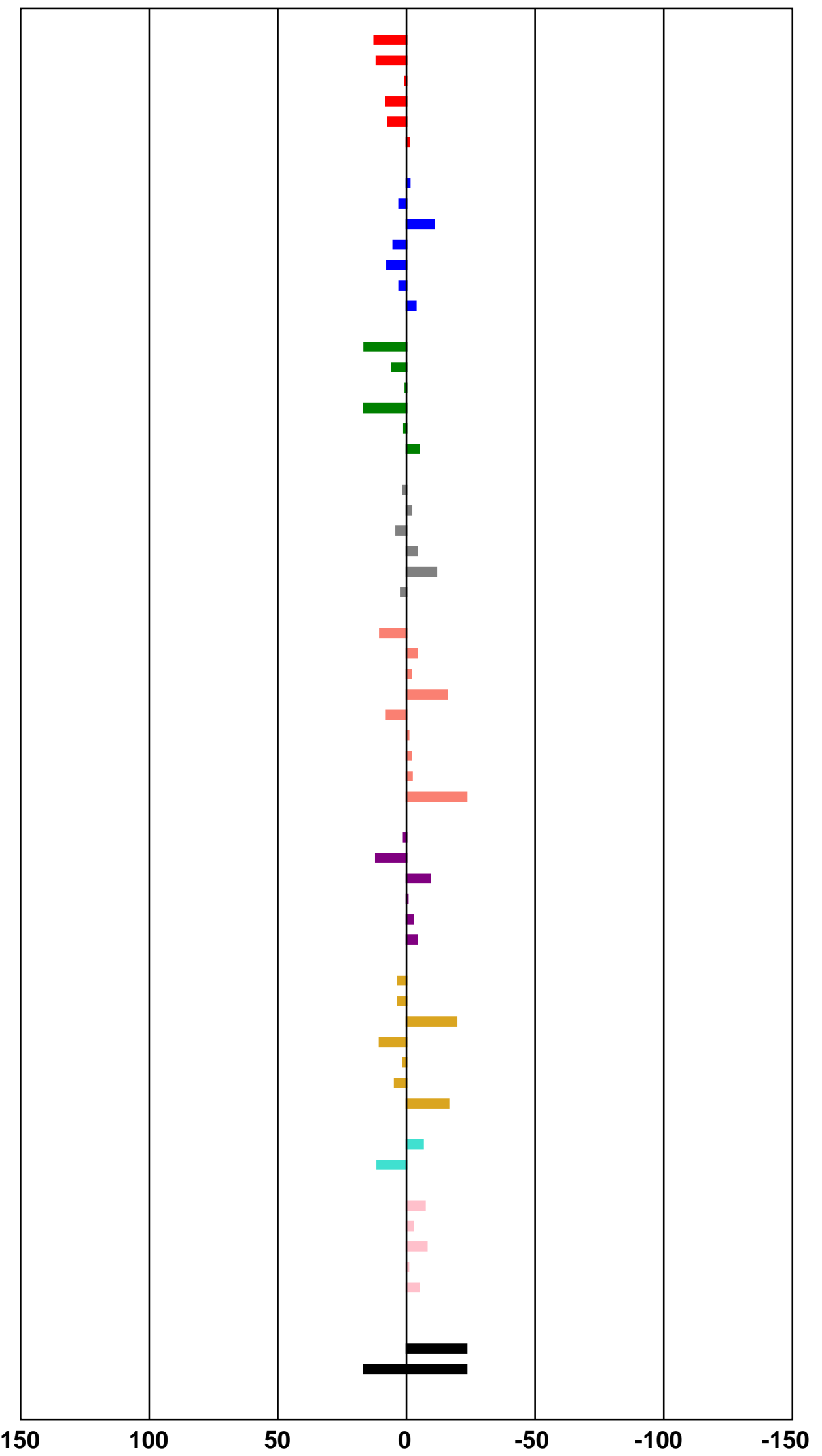


unor a \begin{tabular}{l|l} 
NSC: $D-816480 / 1$ & Conc: $1.00 E-5$ Molar
\end{tabular} Test Date: Jun 17, 2019 One Dose Mean Graph

\section{Panel/Cell Line}

Leukemia

CCRF-CEM

HL-60(TB)

$\mathrm{K}-562$

MOLT-4

RPMI-8226

SR

Non-Small Cell Lung Cancer

A549/ATCC

HOP-62

HOP-92

$\mathrm{NCl}-\mathrm{H} 23$

$\mathrm{NCl}-\mathrm{H} 322 \mathrm{M}$

$\mathrm{NCl}-\mathrm{H} 460$

$\mathrm{NCl}-\mathrm{H} 522$

Colon Cancer

HCC-2998

HCT-116

HCT-15

HT29

KM12

SW-620

CNS Cancer

SF-268

SF-295

SF-539

SNB-19

SNB-75

U251

Melanoma

LOX IMVI

MALME-3M

M14

MDA-MB-435

SK-MEL-2

SK-MEL-28

SK-MEL-5

UACC-257

UACC-62

Ovarian Cancer

IGROV1

OVCAR-3

OVCAR-4

OVCAR-5

OVCAR-8

NCI/ADR-RES

Renal Cancer

786-0

ACHN

CAKI-1

RXF 393

SN12C

TK-10

UO-31

Prostate Cancer

PC-3

DU-145

Breast Cancer

MCF7

MDA-MB-231/ATCC

BT-549

MDA-MB-468

Mean

Delta

Range

\section{Growth Percent}

17.44

7.90

20.15

40.85

27.86

41.48

31.37

34.05

37.06

39.95

42.29

18.04

10.89

39.88

22.35

24.42

12.53

20.49

26.22

53.53

12.20

$-22.71$

39.19

$-13.35$

25.04

44.46

47.32

19.02

$-35.19$

38.99

59.26

17.31

69.40

40.17

33.10

$-32.12$

49.97

38.32

33.75

8.53

51.05

49.78

38.06

$-26.98$

48.34

61.86

49.77

19.58

13.49

15.71

34.81

49.46

6.43

27.41

62.60

104.59
Mean Growth Percent - Growth Percent

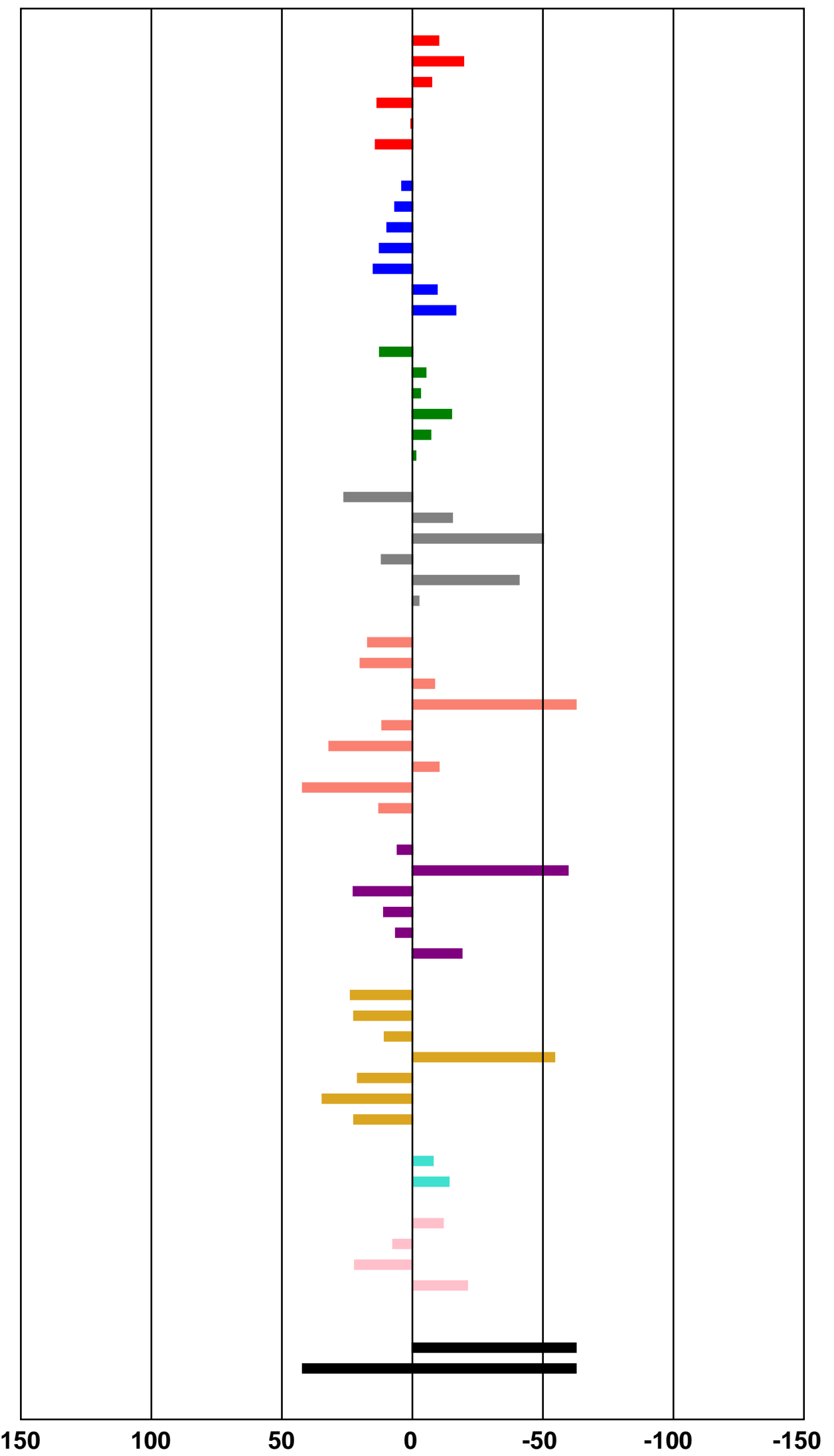




\section{Panel/Cell Line}

Leukemia

CCRF-CEM

HL-60(TB)

$\mathrm{K}-562$

MOLT-4

RPMI-8226

Non-Small Cell Lung Cancer

A549/ATCC

EKVX

HOP-62

HOP-92

$\mathrm{NCl}-\mathrm{H} 226$

$\mathrm{NCl}-\mathrm{H} 23$

$\mathrm{NCl}-\mathrm{H} 322 \mathrm{M}$

$\mathrm{NCl}-\mathrm{H} 460$

$\mathrm{NCl}-\mathrm{H} 522$

Colon Cancer

COLO 205

HCC-2998

HCT-116

HCT-15

HT29

KM12

SW-620

CNS Cancer

SF-268

SF-295

SF-539

SNB-19

SNB-75

Melanoma

LOX IMVI

MALME-3M

M14

MDA-MB-435

SK-MEL-2

SK-MEL-28

SK-MEL-5

UACC-257

UACC-62

Ovarian Cancer

IGROV1

OVCAR-3

OVCAR-4

OVCAR-5

OVCAR-8

NCI/ADR-RES

SK-OV-3

Renal Cancer

786-0

A498

ACHN

CAKI-1

RXF 393

SN12C

TK-10

UO-31

Prostate Cancer

PC-3

DU-145

Breast Cancer

MCF7

MDA-MB-231/ATCC

HS 578T

BT-549

T-47D

MDA-MB-468

\section{Growth Percent}

19.59

8.62

15.37

29.46

19.28

21.39

32.16

33.09

42.02

47.13

24.79

45.69

10.46

$-11.53$

$-6.19$

32.04

19.73

22.30

$-3.16$

30.80

17.29

52.21

5.73

$-14.72$

49.28

$-14.42$

42.81

44.97

24.83

2.08

37.99

62.49

6.59

48.24

28.32

27.27

$-8.13$

39.11

34.40

21.67

$-17.04$

35.14

43.17

$-14.50$

44.52

32.50

$-9.55$

49.89

44.03

41.84

24.91

16.25

15.48

36.20

11.27

36.32

27.38

$-23.86$

23.02

46.88

86.35
Mean Growth Percent - Growth Percent

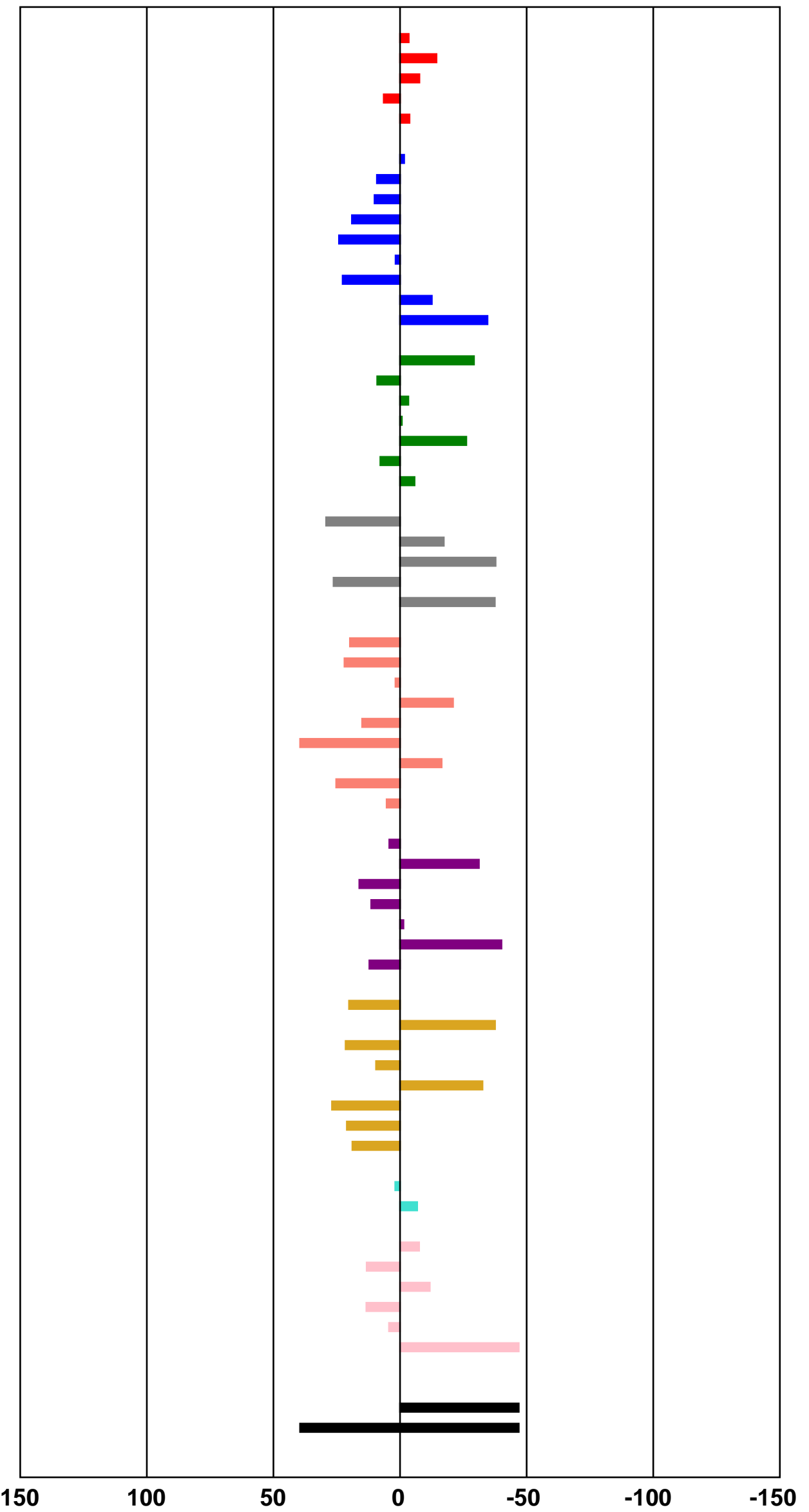




\section{Panel/Cell Line}

Leukemia

CCRF-CEM

HL-60(TB)

K-562

MOLT-4

RPMI-8226

Non-Small Cell Lung Cancer

A549/ATCC

EKVX

HOP-62

HOP-92

$\mathrm{NCl}-\mathrm{H} 226$

$\mathrm{NCl}-\mathrm{H} 23$

$\mathrm{NCl}-\mathrm{H} 322 \mathrm{M}$

$\mathrm{NCl}-\mathrm{H} 460$

$\mathrm{NCl}-\mathrm{H} 522$

Colon Cancer

COLO 205

HCC-2998

HCT-116

HCT-15

HT29

KM12

SW-620

CNS Cancer

SF-268

SF-295

SF-539

SNB-19

SNB-75

Melanoma

LOX IMVI

MALME-3M

M14

MDA-MB-435

SK-MEL-2

SK-MEL-28

SK-MEL-5

UACC-257

UACC-62

Ovarian Cancer

IGROV1

OVCAR-3

OVCAR-4

OVCAR-5

OVCAR-8

NCI/ADR-RES

SK-OV-3

Renal Cancer

786-0

A498

ACHN

CAKI-1

RXF 393

SN12C

TK-10

UO-31

Prostate Cancer

PC-3

DU-145

Breast Cancer

MCF7

MDA-MB-231/ATCC

HS 578T

BT-549

T-47D

MDA-MB-468

\section{Growth Percent}

19.86

0.94

12.30

28.55

21.17

30.10

34.39

32.46

42.01

58.65

25.19

39.72

13.50

$-15.79$

1.76

41.51

23.12

21.06

$-6.67$

38.45

17.92

52.14

10.33

$-15.53$

44.94

$-7.32$

40.32

35.31

19.55

3.28

31.90

58.46

17.44

52.91

24.76

27.40

$-6.70$

49.10

35.81

24.51

$-20.80$

36.34

42.56

$-16.08$

47.51

32.07

$-7.66$

47.61

45.49

48.64

29.93

16.61

12.98

37.75

16.07

27.46

27.78

$-25.39$

23.75

49.14

84.04
Mean Growth Percent - Growth Percent

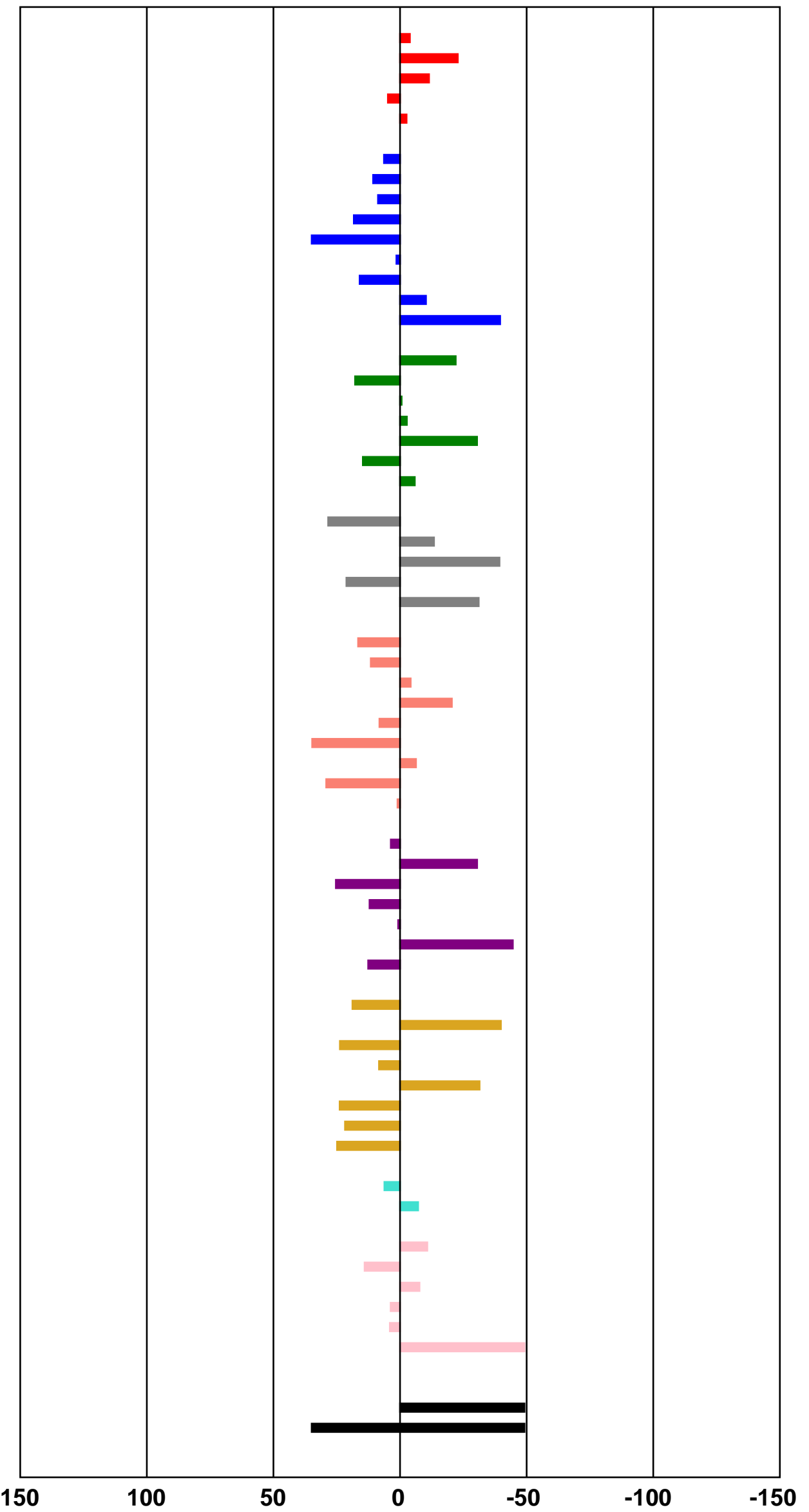


NSC: D-824737/1

\section{Panel/Cell Line}

Leukemia

CCRF-CEM

HL-60(TB)

K-562

RPMI-8226

Non-Small Cell Lung Cancer

A549/ATCC

EKVX

HOP-62

HOP-92

$\mathrm{NCl}-\mathrm{H} 226$

$\mathrm{NCl}-\mathrm{H} 23$

$\mathrm{NCl}-\mathrm{H} 322 \mathrm{M}$

$\mathrm{NCl}-\mathrm{H} 460$

$\mathrm{NCl}-\mathrm{H} 522$

Colon Cancer

COLO 205

HCC-2998

HCT-116

HCT-15

HT29

KM12

SW-620

CNS Cancer

SF-268

SF-295

SF-539

SNB-19

SNB-75

U251

Melanoma

LOX IMVI

MALME-3M

M14

MDA-MB-435

SK-MEL-2

SK-MEL-28

SK-MEL-5

UACC-257

UACC-62

Ovarian Cancer

IGROV1

OVCAR-3

OVCAR-4

OVCAR-5

OVCAR-8

NCI/ADR-RES

SK-OV-3

Renal Cancer

786-0

A498

$\mathrm{ACHN}$

CAKI-1

RXF 393

SN12C

TK-10

UO-31

Prostate Cancer

PC-3

DU-145

Breast Cancer

MCF7

MDA-MB-231/ATCC

HS $578 \mathrm{~T}$

BT-549

T-47D

Mean

Delta

Range

\section{Growth Percent}

12.78

2.04

9.52

17.76

38.44

35.14

31.94

20.45

40.88

29.35

48.14

16.54

$-1.89$

0.34

46.25

15.27

23.93

3.59

20.80

11.33

50.99

15.53

$-0.02$

24.13

$-25.81$

22.90

35.41

58.17

16.25

$-21.47$

58.80

68.45

16.41

63.82

29.16

26.38

$-9.95$

41.70

37.20

33.48

18.90

34.26

28.37

$-3.40$

37.10

29.81

13.80

39.69

45.88

35.50

26.30

20.45

14.19

23.25

1.30

26.60

25.02

24.23

50.04

94.26
Mean Growth Percent - Growth Percent

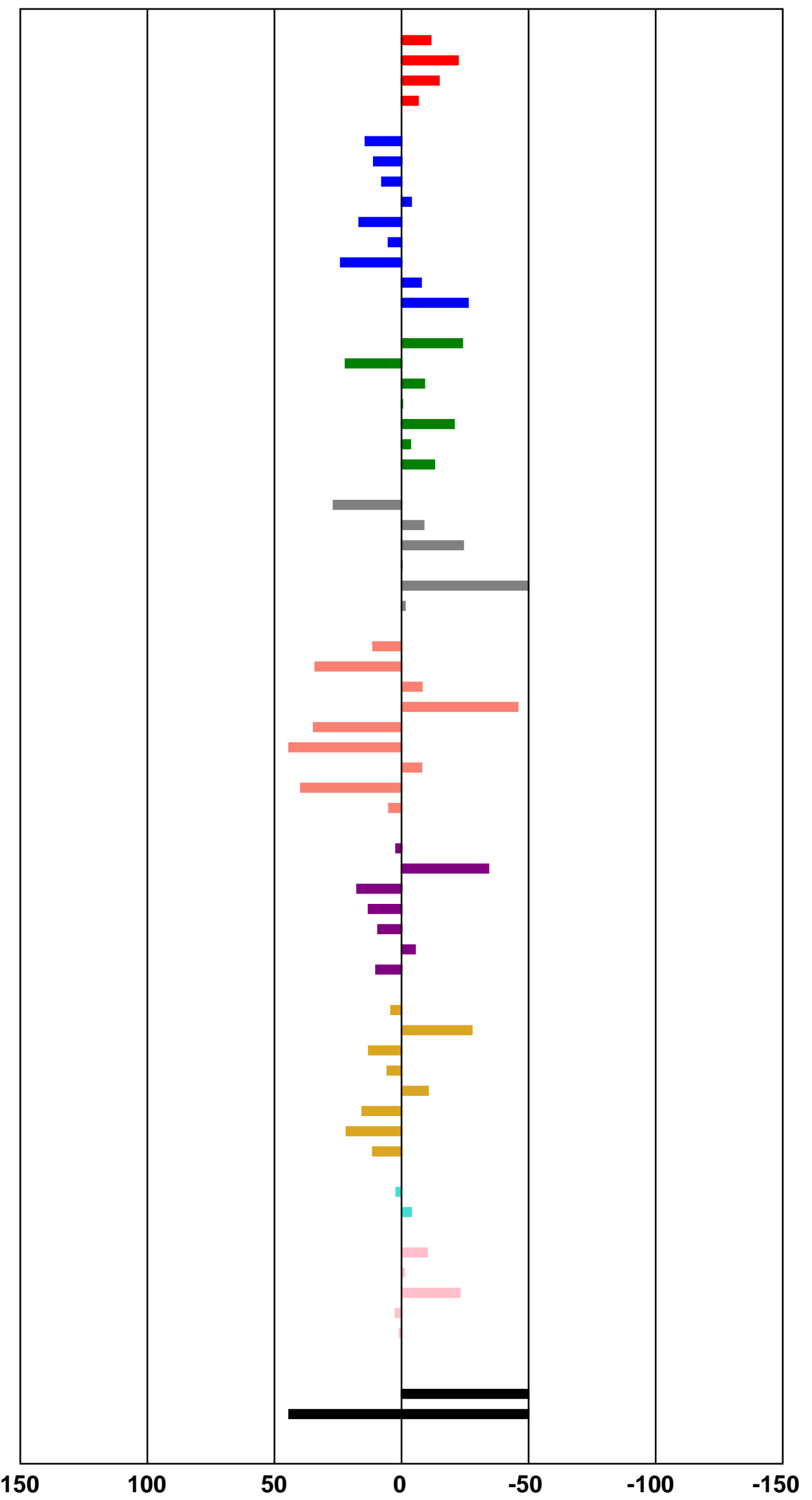




\section{Panel/Cell Line}

Leukemia

CCRF-CEM

HL-60(TB)

$\mathrm{K}-562$

MOLT-4

RPMI-8226

Non-Small Cell Lung Cancer

A549/ATCC

EKVX

HOP-62

HOP-92

$\mathrm{NCl}-\mathrm{H} 226$

$\mathrm{NCl}-\mathrm{H} 23$

$\mathrm{NCl}-\mathrm{H} 322 \mathrm{M}$

$\mathrm{NCl}-\mathrm{H} 460$

$\mathrm{NCl}-\mathrm{H} 522$

Colon Cancer

COLO 205

HCC-2998

HCT-116

HCT-15

HT29

KM12

SW-620

CNS Cancer

SF-268

SF-295

SF-539

SNB-19

SNB-75

Melanoma

LOX IMVI

MALME-3M

M14

MDA-MB-435

SK-MEL-2

SK-MEL-28

SK-MEL-5

UACC-257

UACC-62

Ovarian Cancer

IGROV1

OVCAR-3

OVCAR-4

OVCAR-5

OVCAR-8

NCI/ADR-RES

SK-OV-3

Renal Cancer

786-0

A498

ACHN

CAKI-1

RXF 393

SN12C

TK-10

UO-31

Prostate Cancer

PC-3

DU-145

Breast Cancer

MCF7

MDA-MB-231/ATCC

HS 578T

BT-549

T-47D

MDA-MB-468

\section{Growth Percent}

14.43

0.81

10.23

17.75

24.85

15.30

28.52

34.10

60.71

58.40

24.28

44.74

7.65

$-11.69$

$-13.73$

25.12

13.25

17.04

$-17.11$

27.03

21.69

49.40

1.80

$-28.61$

44.32

0.00

46.48

46.32

4.78

0.57

45.34

50.53

24.81

45.04

26.44

27.71

$-20.86$

44.74

21.97

12.93

0.31

32.36

33.69

$-17.80$

40.38

34.66

$-10.71$

48.41

54.25

46.50

24.74

14.30

11.06

33.93

11.93

28.77

32.38

$-6.60$

21.72

50.33

89.32
Mean Growth Percent - Growth Percent

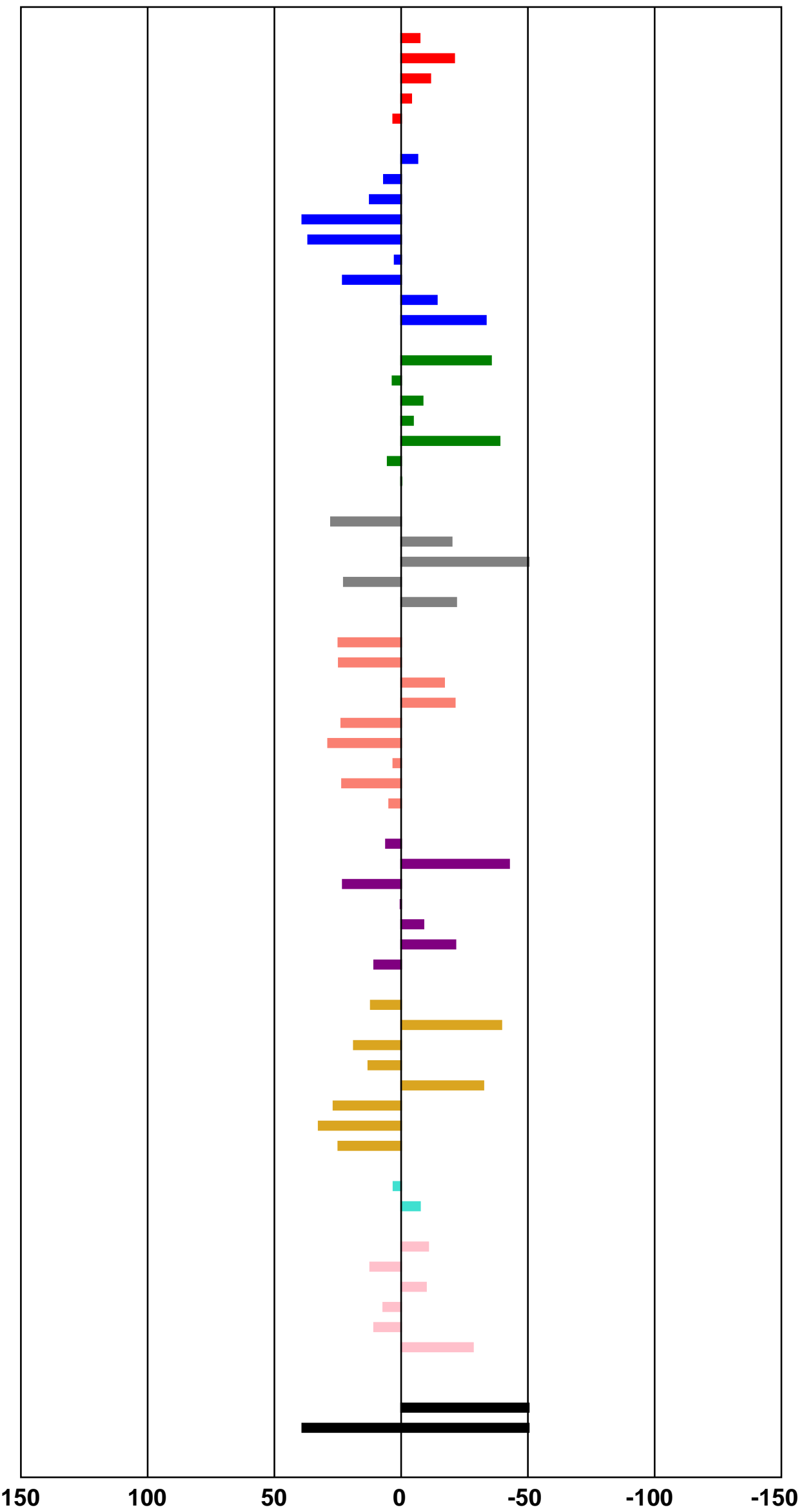




\section{Panel/Cell Line}

Leukemia

CCRF-CEM

HL-60(TB)

$\mathrm{K}-562$

MOLT-4

RPMI-8226

Non-Small Cell Lung Cancer

A549/ATCC

EKVX

HOP-62

HOP-92

$\mathrm{NCl}-\mathrm{H} 226$

$\mathrm{NCl}-\mathrm{H} 23$

$\mathrm{NCl}-\mathrm{H} 322 \mathrm{M}$

$\mathrm{NCl}-\mathrm{H} 460$

$\mathrm{NCl}-\mathrm{H} 522$

Colon Cancer

COLO 205

HCC-2998

HCT-116

HCT-15

HT29

KM12

SW-620

CNS Cancer

SF-268

SF-295

SF-539

SNB-19

SNB-75

Melanoma

LOX IMVI

MALME-3M

M14

MDA-MB-435

SK-MEL-2

SK-MEL-28

SK-MEL-5

UACC-257

UACC-62

Ovarian Cancer

IGROV1

OVCAR-3

OVCAR-4

OVCAR-5

OVCAR-8

NCI/ADR-RES

SK-OV-3

Renal Cancer

786-0

A498

ACHN

CAKI-1

RXF 393

SN12C

TK-10

UO-31

Prostate Cancer

PC-3

DU-145

Breast Cancer

MCF7

MDA-MB-231/ATCC

HS 578T

BT-549

T-47D

MDA-MB-468

\section{Growth Percent}

61.12

17.08

13.17

37.48

71.14

50.62

68.53

52.38

62.13

80.20

71.19

86.19

38.43

31.23

68.36

76.93

40.77

25.48

15.92

52.87

27.16

63.31

28.61

44.07

62.82

15.44

52.48

50.85

37.36

2.11

40.70

61.75

62.83

63.11

32.84

45.35

16.14

60.49

88.19

69.97

27.97

71.53

69.11

$-10.05$

77.25

40.89

72.97

71.48

88.59

70.10

67.96

78.15

27.89

61.04

35.19

45.68

52.02

$-9.07$

49.75

59.80

98.64
Mean Growth Percent - Growth Percent

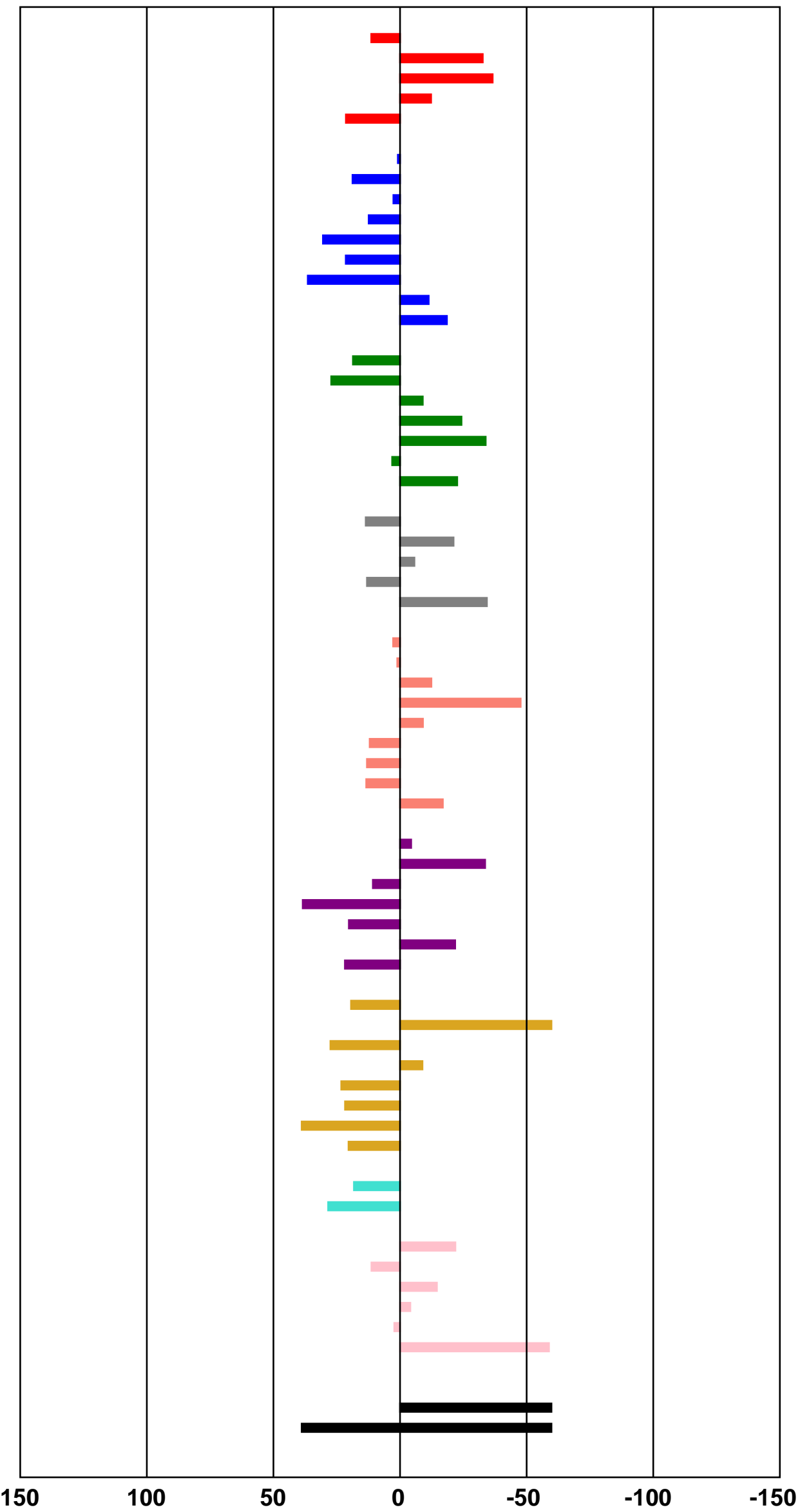




\section{Panel/Cell Line}

Leukemia

CCRF-CEM

HL-60(TB)

K-562

MOLT-4

RPMI-8226

Non-Small Cell Lung Cancer

A549/ATCC

EKVX

HOP-62

HOP-92

$\mathrm{NCl}-\mathrm{H} 226$

$\mathrm{NCl}-\mathrm{H} 23$

$\mathrm{NCl}-\mathrm{H} 322 \mathrm{M}$

$\mathrm{NCl}-\mathrm{H} 460$

$\mathrm{NCl}-\mathrm{H} 522$

Colon Cancer

COLO 205

HCC-2998

HCT-116

HCT-15

HT29

KM12

SW-620

CNS Cancer

SF-268

SF-295

SF-539

SNB-19

SNB-75

Melanoma

LOX IMVI

MALME-3M

M14

MDA-MB-435

SK-MEL-2

SK-MEL-28

SK-MEL-5

UACC-257

UACC-62

Ovarian Cancer

IGROV1

OVCAR-3

OVCAR-4

OVCAR-5

OVCAR-8

NCI/ADR-RES

SK-OV-3

Renal Cancer

786-0

A498

ACHN

CAKI-1

RXF 393

SN12C

TK-10

UO-31

Prostate Cancer

PC-3

DU-145

Breast Cancer

MCF7

MDA-MB-231/ATCC

HS 578T

BT-549

T-47D

MDA-MB-468

\section{Growth Percent}

19.30

$-4.27$

10.25

27.55

20.45

24.93

33.87

32.63

40.79

55.27

29.70

39.16

11.86

$-17.73$

$-2.39$

45.94

19.52

20.73

$-15.25$

33.81

19.26

50.32

5.84

$-19.79$

35.03

$-5.71$

43.70

30.91

5.11

$-17.09$

34.42

57.95

10.23

55.53

30.12

26.83

$-19.65$

46.26

29.42

20.44

$-15.23$

36.28

39.00

$-18.19$

46.44

30.87

$-10.58$

41.12

51.54

47.45

23.93

9.63

11.65

33.57

10.00

38.29

30.93

$-23.93$

21.52

45.45

81.88
Mean Growth Percent - Growth Percent

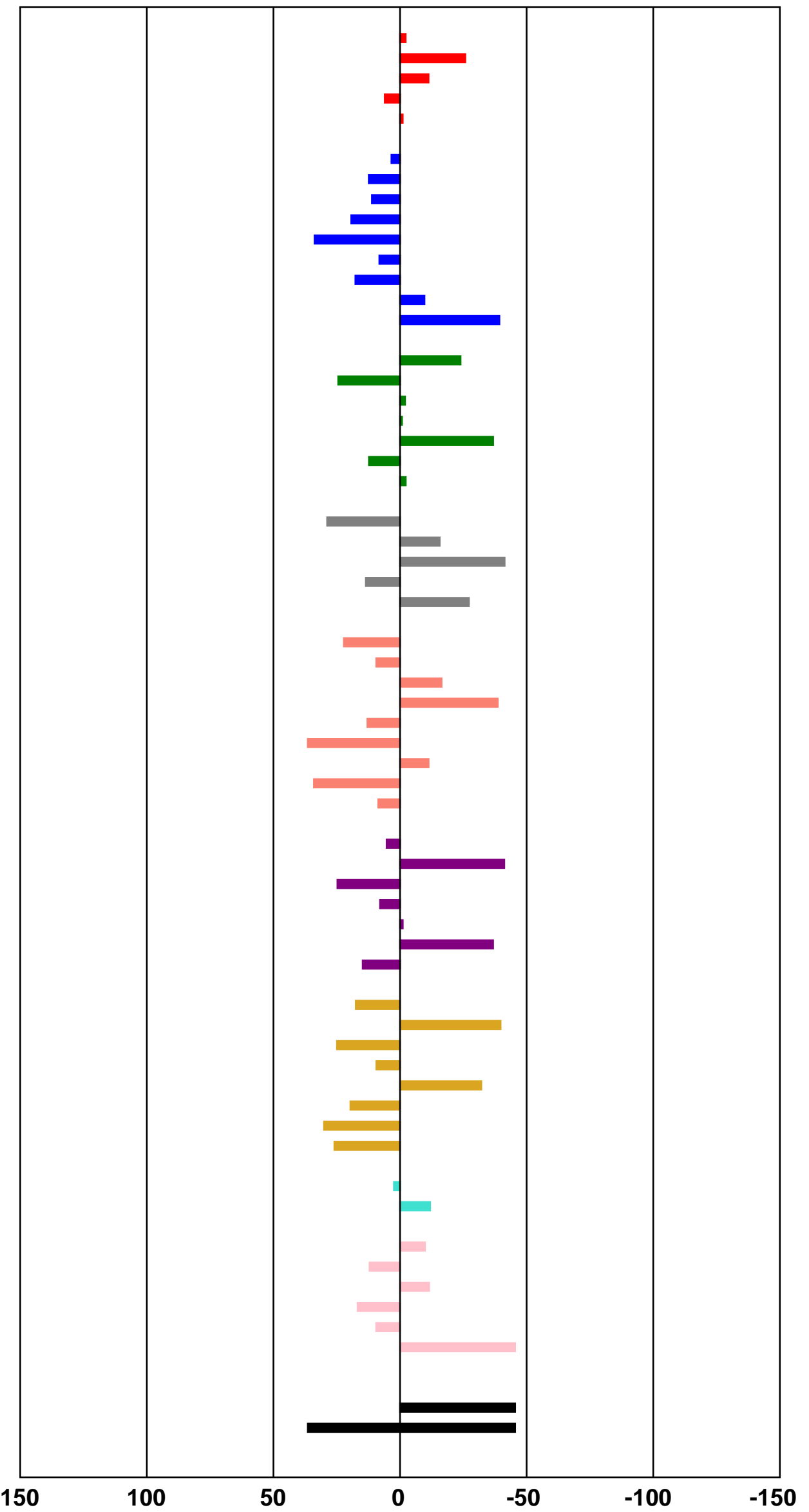


NSC: D-823375/1

\section{Panel/Cell Line}

Leukemia

CCRF-CEM

HL-60(TB)

$\mathrm{K}-562$

MOLT-4

RPMI-8226

Non-Small Cell Lung Cancer

A549/ATCC

EKVX

HOP-62

HOP-92

$\mathrm{NCl}-\mathrm{H} 226$

$\mathrm{NCl}-\mathrm{H} 23$

$\mathrm{NCl}-\mathrm{H} 322 \mathrm{M}$

$\mathrm{NCl}-\mathrm{H} 460$

$\mathrm{NCl}-\mathrm{H} 522$

Colon Cancer

COLO 205

HCC-2998

HCT-116

HCT-15

HT29

KM12

SW-620

CNS Cancer

SF-268

SF-295

SF-539

SNB-19

SNB-75

Melanoma

LOX IMVI

MALME-3M

M14

MDA-MB-435

SK-MEL-2

SK-MEL-28

SK-MEL-5

UACC-257

UACC-62

Ovarian Cancer

IGROV1

OVCAR-3

OVCAR-4

OVCAR-5

OVCAR-8

NCI/ADR-RES

SK-OV-3

Renal Cancer

786-0

A498

ACHN

CAKI-1

RXF 393

SN12C

TK-10

UO-31

Prostate Cancer

PC-3

DU-145

Breast Cancer

MCF7

MDA-MB-231/ATCC

HS 578T

BT-549

T-47D

MDA-MB-468

\section{Growth Percent}

23.49

$-1.17$

9.36

30.09

12.44

27.13

26.99

33.71

29.62

47.60

21.20

42.67

12.34

$-7.94$

$-10.05$

45.04

19.40

19.53

$-10.36$

42.78

19.66

51.97

10.29

$-13.64$

46.78

$-7.43$

39.38

42.63

25.57

4.45

38.54

65.97

$-3.90$

49.77

24.05

29.46

$-1.67$

43.20

37.59

28.21

$-10.85$

30.71

43.36

$-15.79$

40.67

27.23

$-3.66$

43.60

43.97

38.49

22.75

14.35

12.08

40.11

14.32

35.48

26.43

$-25.60$

22.80

48.40

91.57
Mean Growth Percent - Growth Percent

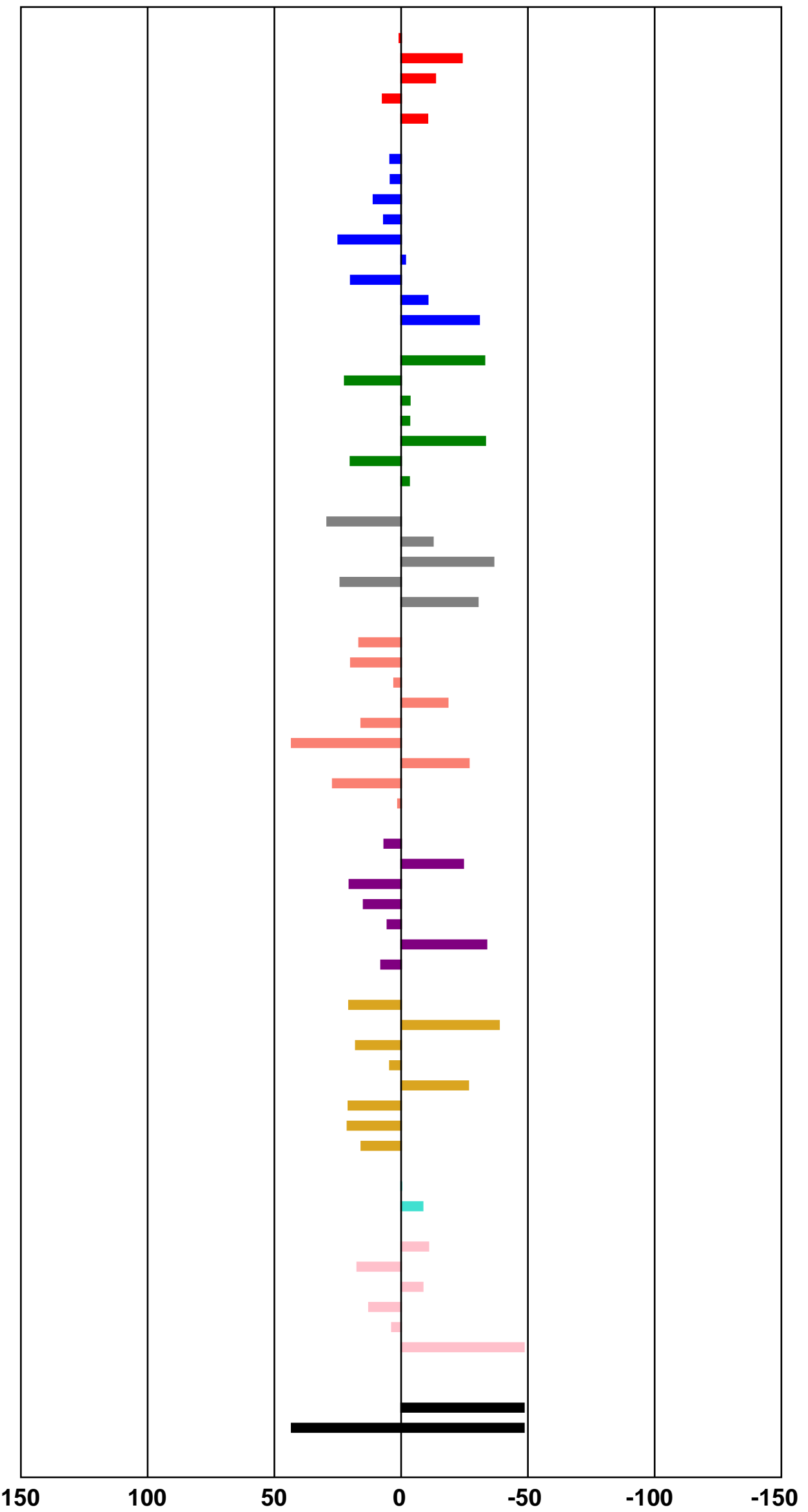




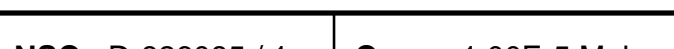
\begin{tabular}{l|l} 
NSC: D-826085 / 1 Conc: $1.00 E-5$ Molar
\end{tabular} Test Date: Sep 28, 2020 Experiment ID: 20090551

\section{Panel/Cell Line}

Leukemia

CCRF-CEM

HL-60(TB)

$\mathrm{K}-562$

MOLT-4

SR

Non-Small Cell Lung Cancer

A549/ATCC

EKVX

HOP-62

HOP-92

$\mathrm{NCl}-\mathrm{H} 226$

$\mathrm{NCl}-\mathrm{H} 23$

$\mathrm{NCl}-\mathrm{H} 322 \mathrm{M}$

$\mathrm{NCl}-\mathrm{H} 460$

$\mathrm{NCl}-\mathrm{H} 522$

Colon Cancer

COLO 205

HCC-2998

HCT-116

HCT-15

HT29

KM12

SW-620

CNS Cancer

SF-268

SF-295

SF-539

SNB-19

SNB-75

U251

Melanoma

LOX IMVI

MALME-3M

M14

SK-MEL-2

SK-MEL-28

SK-MEL-5

UACC-257

UACC-62

Ovarian Cancer

IGROV1

OVCAR-3

OVCAR-4

OVCAR-5

OVCAR-8

$\mathrm{NCl} / \mathrm{ADR}$-RES

SK-OV-3

Renal Cancer

786-0

A498

ACHN

CAKI-1

RXF 393

SN12C

TK-10

UO-31

Prostate Cancer

PC-3

DU-145

Breast Cancer

MCF7

MDA-MB-231/ATCC

HS 578T

BT-549

T-47D

MDA-MB-468

\section{Growth Percent}

69.98

84.50

55.53

72.48

73.26

73.39

82.68

71.35

52.69

80.34

75.03

91.15

79.02

63.57

103.40

97.10

71.62

72.85

96.61

77.79

79.12

84.01

71.39

87.67

78.96

73.76

76.54

88.95

77.12

84.11

88.76

89.75

69.70

82.94

62.48

54.58

83.16

63.42

93.58

79.38

75.25

75.12

87.45

78.21

84.48

51.49

85.32

81.64

96.52

70.37

68.03

90.18

71.96

80.29

54.91

92.98

55.23

59.43

77.11

25.62

51.91
Mean Growth Percent - Growth Percent

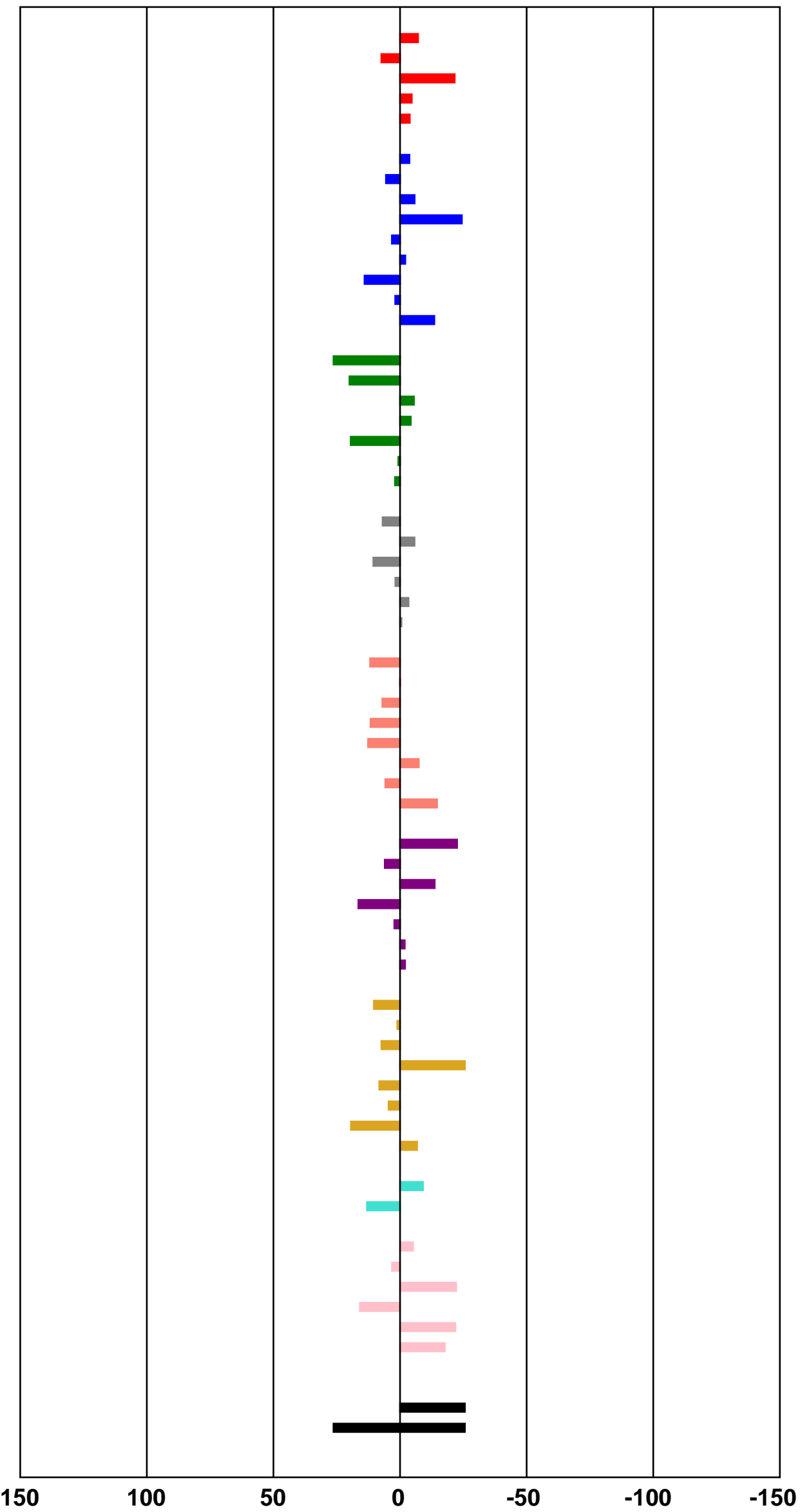


NSC: D-823377/1

\section{Panel/Cell Line}

Leukemia

CCRF-CEM

HL-60(TB)

$\mathrm{K}-562$

MOLT-4

RPMI-8226

$\mathrm{SR}$

Non-Small Cell Lung Cancer

A549/ATCC

EKVX

HOP-62

HOP-92

$\mathrm{NCl}-\mathrm{H} 226$

$\mathrm{NCl}-\mathrm{H} 23$

$\mathrm{NCl}-\mathrm{H} 322 \mathrm{M}$

$\mathrm{NCl}-\mathrm{H} 460$

$\mathrm{NCl}-\mathrm{H} 522$

Colon Cancer

COLO 205

HCC-2998

HCT-116

HCT-15

HT29

KM12

SW-620

CNS Cancer

SF-268

SF-539

SNB-19

SNB-75

U251

Melanoma

LOX IMVI

MALME-3M

M14

MDA-MB-435

SK-MEL-2

SK-MEL-28

SK-MEL-5

UACC-257

UACC-62

Ovarian Cancer

IGROV1

OVCAR-3

OVCAR-4

OVCAR-5

OVCAR-8

NCI/ADR-RES

SK-OV-3

Renal Cancer

786-0

A498

ACHN

CAKI-1

RXF 393

SN12C

TK-10

UO-31

Prostate Cancer

PC-3

DU-145

Breast Cancer

MCF7

MDA-MB-231/ATCC

HS 578T

BT-549

T-47D

MDA-MB-468

\section{Growth Percent}

19.33

1.00

18.25

28.33

18.07

10.95

41.49

41.05

39.71

44.39

64.27

37.99

47.39

16.31

13.44

16.21

54.91

21.25

23.68

14.03

30.07

23.74

44.61

30.70

1.19

46.47

$-13.06$

26.61

41.47

34.85

4.25

$-33.82$

36.70

63.55

6.16

63.99

27.39

32.64

13.38

46.76

51.77

38.38

19.38

45.61

43.99

$-3.02$

38.45

25.04

$-1.92$

42.89

67.17

39.95

42.50

40.75

20.27

52.98

27.36

17.25

31.89

2.58

29.05

62.87

100.99
Mean Growth Percent - Growth Percent

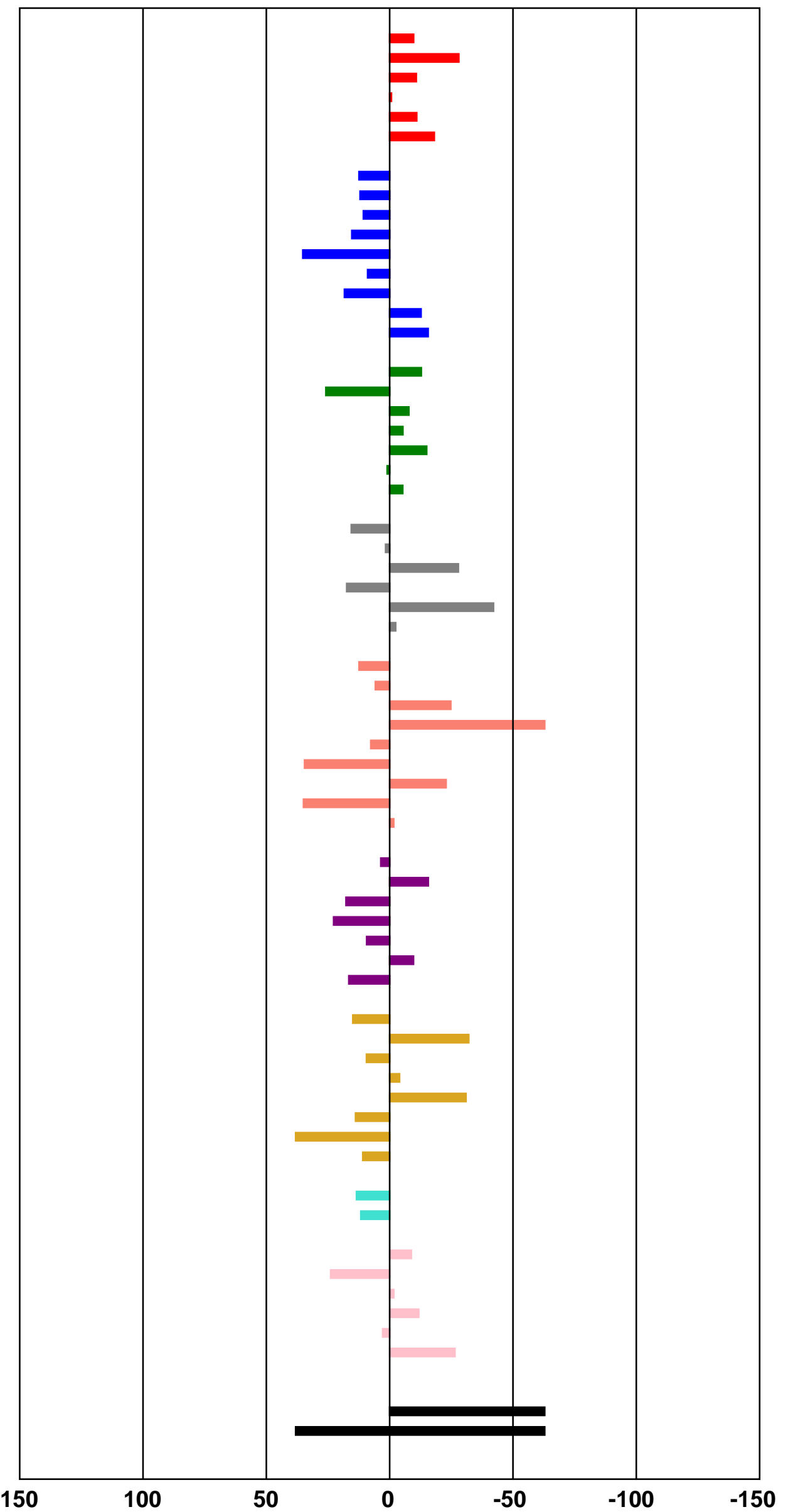


NSC: D-824738/1

\section{Panel/Cell Line}

Leukemia

CCRF-CEM

HL-60(TB)

$\mathrm{K}-562$

MOLT-4

RPMI-8226

$\mathrm{SR}$

Non-Small Cell Lung Cancer

A549/ATCC

EKVX

HOP-62

HOP-92

$\mathrm{NCl}-\mathrm{H} 226$

$\mathrm{NCl}-\mathrm{H} 23$

$\mathrm{NCl}-\mathrm{H} 322 \mathrm{M}$

$\mathrm{NCl}-\mathrm{H} 460$

$\mathrm{NCl}-\mathrm{H} 522$

Colon Cancer

COLO 205

HCC-2998

HCT-116

HCT-15

HT29

KM12

SW-620

CNS Cancer

SF-268

SF-539

SNB-19

SNB-75

U251

Melanoma

LOX IMVI

MALME-3M

M14

MDA-MB-435

SK-MEL-2

SK-MEL-28

SK-MEL-5

UACC-257

UACC-62

Ovarian Cancer

IGROV1

OVCAR-3

OVCAR-4

OVCAR-5

OVCAR-8

NCI/ADR-RES

SK-OV-3

Renal Cancer

786-0

A498

ACHN

CAKI-1

RXF 393

SN12C

TK-10

UO-31

Prostate Cancer

PC-3

DU-145

Breast Cancer

MCF7

MDA-MB-231/ATCC

HS 578T

BT-549

T-47D

\section{Growth Percent}

20.00

0.30

12.00

44.10

31.04

16.54

45.18

58.72

39.77

40.41

40.29

52.76

59.37

24.77

0.74

43.52

67.37

21.47

26.21

5.45

24.01

21.02

49.73

24.84

25.12

31.73

$-8.98$

30.21

35.78

55.66

29.13

$-11.96$

38.99

63.18

31.07

50.61

33.23

39.26

5.34

52.47

72.11

56.21

26.02

52.04

32.96

$-7.01$

34.16

33.52

44.83

60.28

60.01

49.64

38.32

70.79

12.91

65.15

$-2.43$

40.49

28.68

34.56

46.52

84.07
Mean Growth Percent - Growth Percent

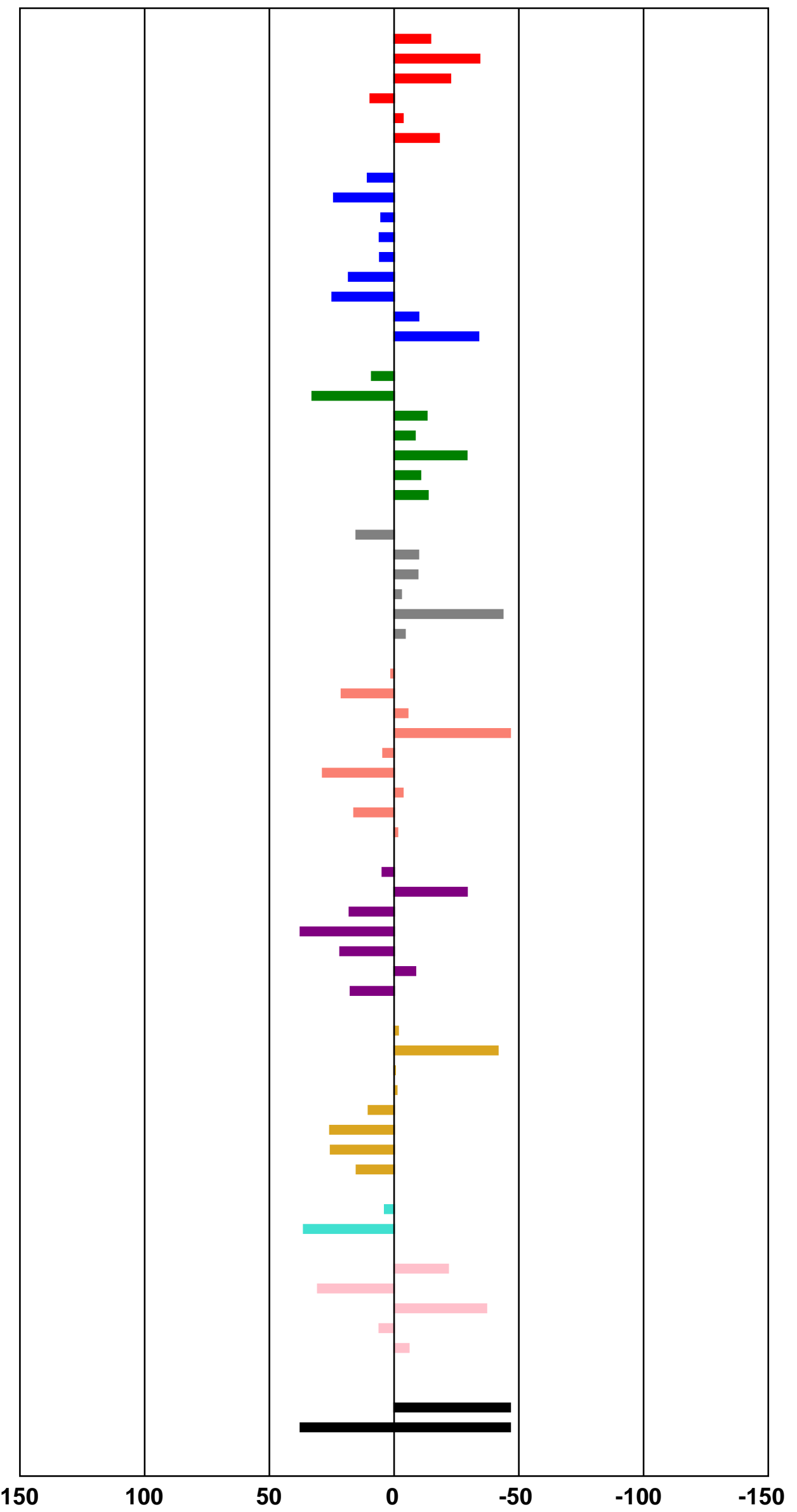


Developmental Therapeutics Program One Dose Mean Graph
NSC: D-824739 / 1

Experiment ID: $20080 S 41$

\section{Panel/Cell Line}

Leukemia

CCRF-CEM

HL-60(TB)

$\mathrm{K}-562$

MOLT-4

RPMI-8226

SR

Non-Small Cell Lung Cancer

A549/ATCC

EKVX

HOP-62

HOP-92

$\mathrm{NCl}-\mathrm{H} 226$

$\mathrm{NCl}-\mathrm{H} 23$

$\mathrm{NCl}-\mathrm{H} 322 \mathrm{M}$

$\mathrm{NCl}-\mathrm{H} 460$

$\mathrm{NCl}-\mathrm{H} 522$

Colon Cancer

COLO 205

HCC-2998

HCT-116

HCT-15

HT29

KM12

SW-620

CNS Cancer

SF-268

SF-295

SF-539

SNB-19

SNB-75

U251

Melanoma

LOX IMVI

MALME-3M

M14

MDA-MB-435

SK-MEL-2

SK-MEL-28

SK-MEL-5

UACC-257

UACC-62

Ovarian Cancer

IGROV 1

OVCAR-3

OVCAR-4

OVCAR-5

OVCAR-8

NCI/ADR-RES

SK-OV-3

Renal Cancer

786-0

A498

ACHN

CAKI-1

RXF 393

SN12C

TK-10

UO-31

Prostate Cancer

PC-3

DU-145

Breast Cancer

MCF7

MDA-MB-231/ATCC

HS 578T

BT-549

T-47D

\section{Growth Percent}

32.20

9.05

9.67

39.09

26.50

16.18

46.67

52.46

40.86

42.50

48.20

63.67

63.16

25.32

7.85

40.07

67.34

19.14

20.00

4.44

19.20

17.23

56.69

20.87

29.18

29.97

$-3.36$

33.01

36.26

50.05

16.50

$-23.64$

39.85

60.80

29.38

62.69

33.72

34.34

2.74

67.78

71.01

49.57

26.37

60.20

35.52

1.50

43.52

32.70

46.63

46.94

63.10

50.10

48.06

70.36

14.34

58.21

14.44

36.14

29.84

35.36

59.00

94.65
Mean Growth Percent - Growth Percent

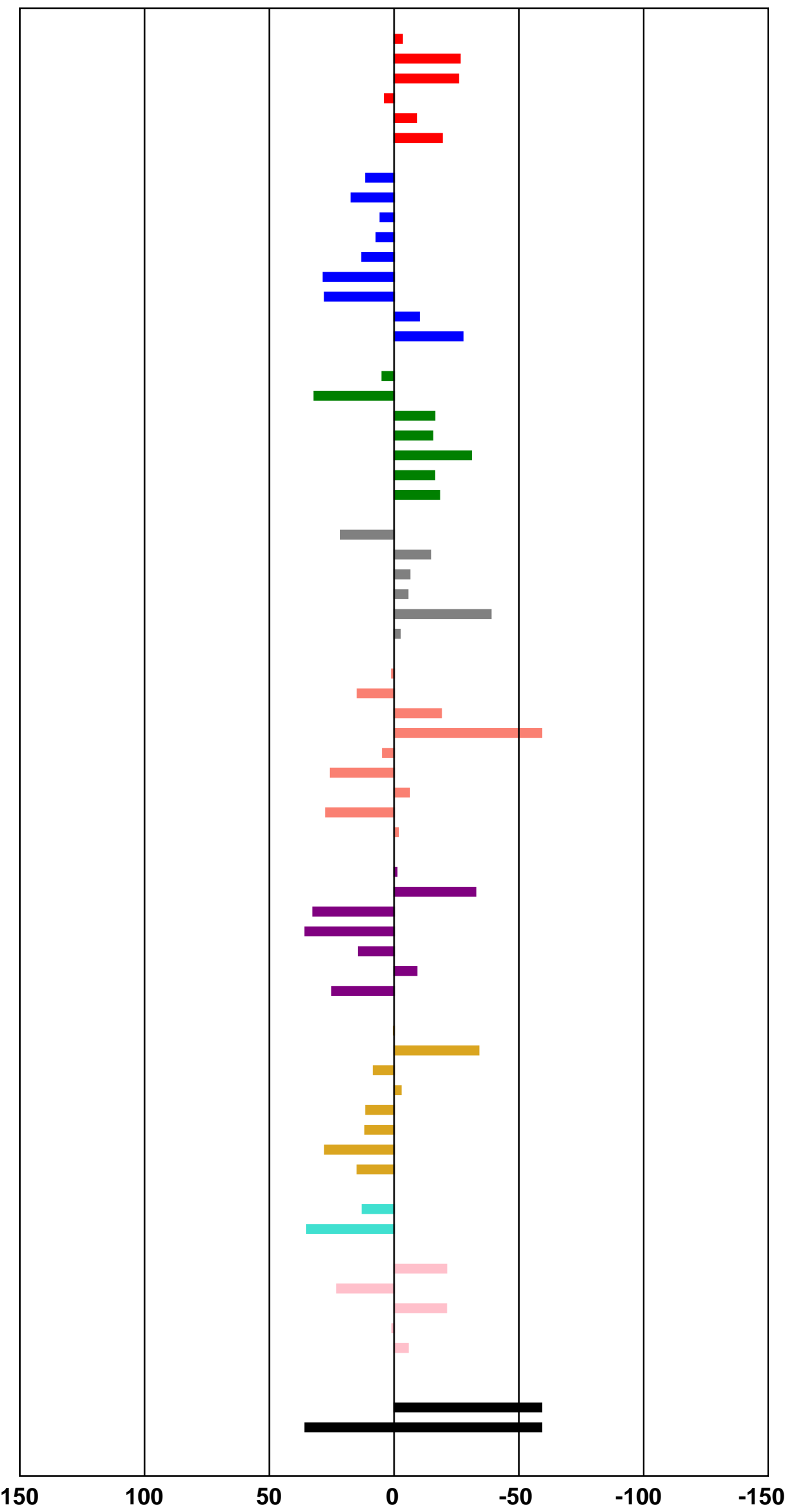




\section{Panel/Cell Line}

Leukemia

CCRF-CEM

HL-60(TB)

$\mathrm{K}-562$

MOLT-4

RPMI-8226

Non-Small Cell Lung Cancer

A549/ATCC

EKVX

HOP-62

HOP-92

$\mathrm{NCl}-\mathrm{H} 226$

$\mathrm{NCl}-\mathrm{H} 23$

$\mathrm{NCl}-\mathrm{H} 322 \mathrm{M}$

$\mathrm{NCl}-\mathrm{H} 460$

$\mathrm{NCl}-\mathrm{H} 522$

Colon Cancer

COLO 205

HCC-2998

HCT-116

HCT-15

HT29

KM12

SW-620

CNS Cancer

SF-268

SF-295

SF-539

SNB-19

SNB-75

Melanoma

LOX IMVI

MALME-3M

M14

MDA-MB-435

SK-MEL-2

SK-MEL-28

SK-MEL-5

UACC-257

UACC-62

Ovarian Cancer

IGROV1

OVCAR-3

OVCAR-4

OVCAR-5

OVCAR-8

NCI/ADR-RES

SK-OV-3

Renal Cancer

786-0

A498

ACHN

CAKI-1

RXF 393

SN12C

TK-10

UO-31

Prostate Cancer

PC-3

DU-145

Breast Cancer

MCF7

MDA-MB-231/ATCC

HS 578T

BT-549

T-47D

MDA-MB-468

\section{Growth Percent}

66.83

64.66

19.84

50.08

52.76

60.38

61.72

66.62

59.34

74.06

73.40

95.69

79.06

42.96

97.18

80.51

65.37

38.38

55.65

74.07

35.19

75.41

53.35

79.20

75.81

42.70

72.01

76.47

50.93

8.10

63.85

85.32

57.68

75.05

43.98

64.76

71.50

52.33

97.55

78.39

41.46

83.36

83.97

16.95

79.44

47.20

81.94

77.46

101.83

60.19

67.64

90.49

39.08

70.17

75.54

63.41

48.26

$-9.07$

63.06

72.13

110.90
Mean Growth Percent - Growth Percent

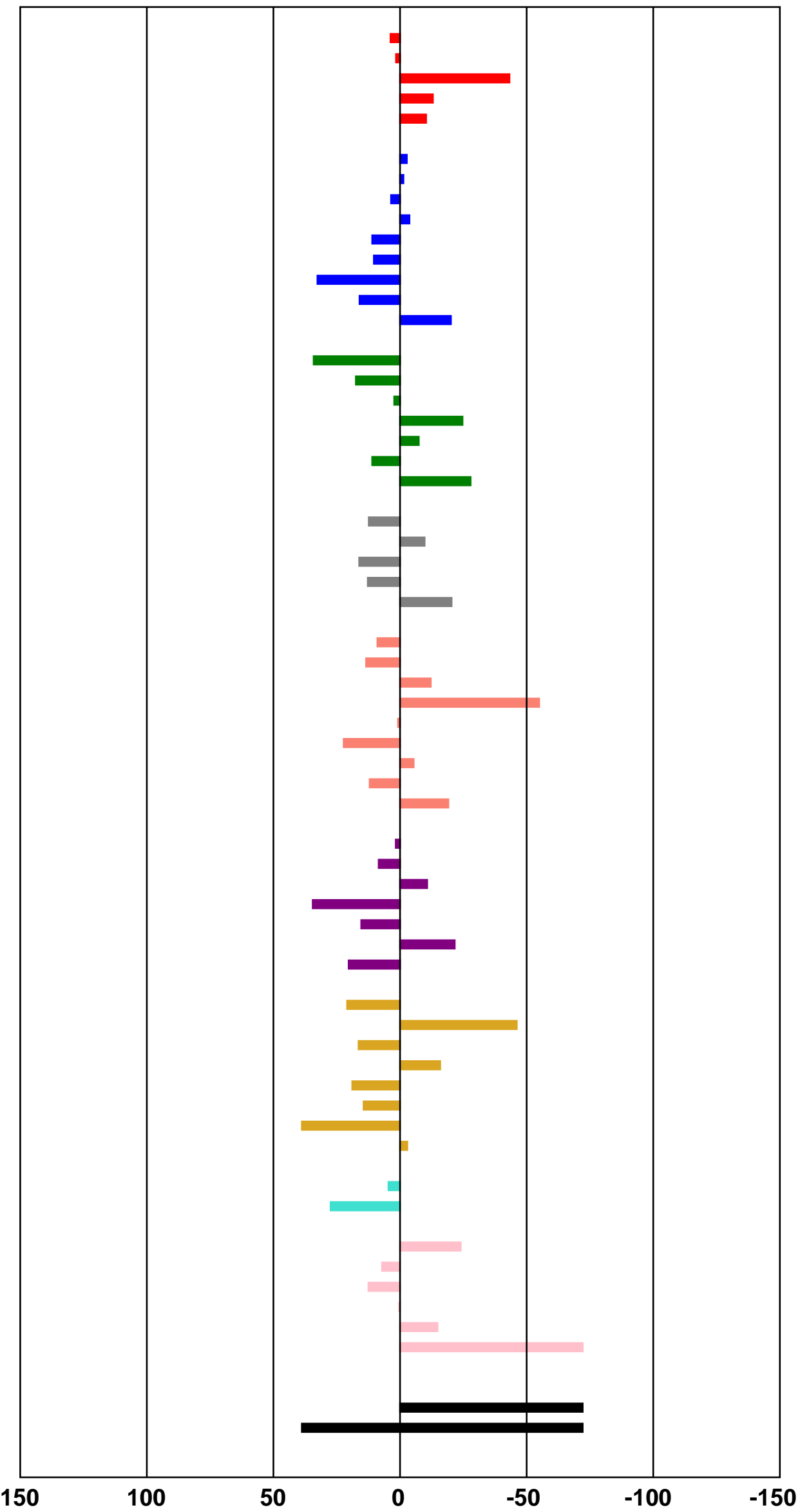


NSC: D-824740/1

\section{Panel/Cell Line}

Leukemia

CCRF-CEM

HL-60(TB)

$\mathrm{K}-562$

MOLT-4

RPMI-8226

$\mathrm{SR}$

Non-Small Cell Lung Cancer

A549/ATCC

EKVX

HOP-62

HOP-92

$\mathrm{NCl}-\mathrm{H} 226$

$\mathrm{NCl}-\mathrm{H} 23$

$\mathrm{NCl}-\mathrm{H} 322 \mathrm{M}$

$\mathrm{NCl}-\mathrm{H} 460$

$\mathrm{NCl}-\mathrm{H} 522$

Colon Cancer

COLO 205

HCC-2998

HCT-116

HCT-15

HT29

KM12

SW-620

CNS Cancer

SF-268

SF-539

SNB-19

SNB-75

U251

Melanoma

LOX IMVI

MALME-3M

M14

MDA-MB-435

SK-MEL-2

SK-MEL-28

SK-MEL-5

UACC-257

UACC-62

Ovarian Cancer

IGROV1

OVCAR-3

OVCAR-4

OVCAR-5

OVCAR-8

NCI/ADR-RES

SK-OV-3

Renal Cancer

786-0

A498

ACHN

CAKI-1

RXF 393

SN12C

TK-10

UO-31

Prostate Cancer

PC-3

DU-145

Breast Cancer

MCF7

MDA-MB-231/ATCC

HS 578T

BT-549

T-47D

\section{Growth Percent}

19.49

1.92

12.18

31.90

20.18

19.55

34.45

33.02

41.75

33.77

36.74

33.64

49.45

18.67

0.25

3.71

38.43

20.93

20.88

2.64

20.71

17.99

55.74

15.60

1.50

25.59

$-15.79$

22.14

34.96

55.83

19.05

$-21.32$

54.19

65.18

15.45

68.98

31.64

24.56

$-15.40$

38.63

36.66

28.63

15.31

47.43

31.38

$-6.34$

37.32

35.41

12.42

41.06

43.79

37.08

33.94

22.98

12.68

24.19

8.89

44.60

33.64

25.93

47.25

90.30
Mean Growth Percent - Growth Percent

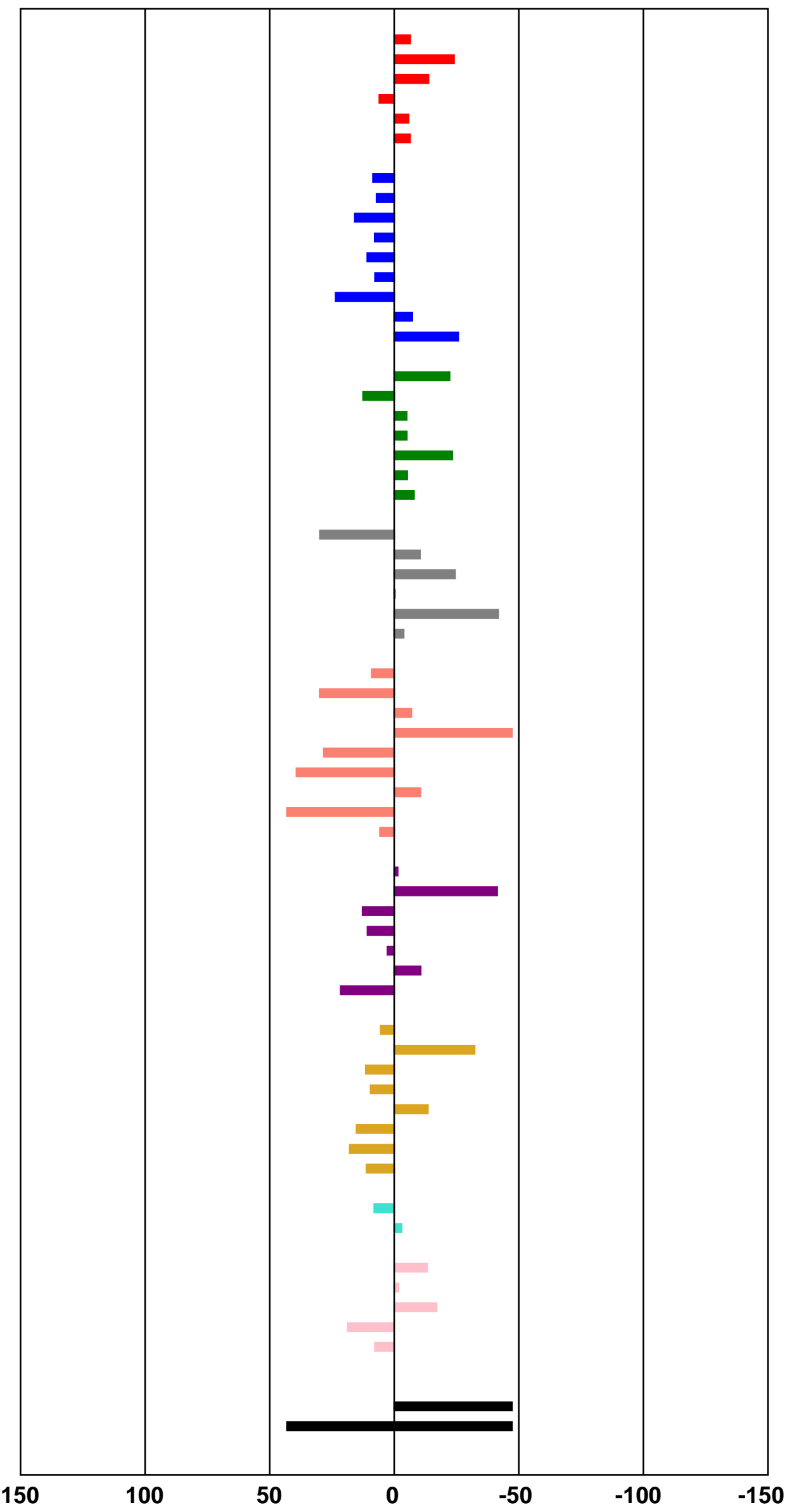


Developmental Therapeutics Program One Dose Mean Graph
NSC: D-824741 / 1

Experiment ID: $20080 S 41$

\section{Panel/Cell Line}

Leukemia

CCRF-CEM

HL-60(TB)

$\mathrm{K}-562$

MOLT-4

RPMI-8226

SR

Non-Small Cell Lung Cancer

A549/ATCC

EKVX

HOP-62

HOP-92

$\mathrm{NCl}-\mathrm{H} 226$

$\mathrm{NCl}-\mathrm{H} 23$

$\mathrm{NCl}-\mathrm{H} 322 \mathrm{M}$

$\mathrm{NCl}-\mathrm{H} 460$

$\mathrm{NCl}-\mathrm{H} 522$

Colon Cancer

COLO 205

HCC-2998

HCT-116

HCT-15

HT29

KM12

SW-620

CNS Cancer

SF-268

SF-539

SNB-19

SNB-75

U251

Melanoma

LOX IMVI

MALME-3M

M14

MDA-MB-435

SK-MEL-2

SK-MEL-28

SK-MEL-5

UACC-257

UACC-62

Ovarian Cancer

IGROV1

OVCAR-3

OVCAR-4

OVCAR-5

OVCAR-8

NCI/ADR-RES

SK-OV-3

Renal Cancer

786-0

A498

ACHN

CAKI-1

RXF 393

SN12C

TK-10

UO-31

Prostate Cancer

PC-3

DU-145

Breast Cancer

MCF7

MDA-MB-231/ATCC

HS 578T

BT-549

T-47D

\section{Growth Percent}

23.65

7.71

17.15

31.89

28.90

25.38

47.32

38.03

40.54

26.68

43.04

43.97

44.53

20.67

1.55

24.28

54.92

28.12

25.38

4.89

29.44

11.95

59.48

22.38

5.30

30.43

$-22.13$

33.55

39.33

52.13

25.18

1.17

52.80

71.33

19.88

62.94

35.15

29.95

10.16

52.52

50.81

39.76

21.94

44.19

30.67

$-6.52$

34.41

31.35

14.76

45.78

47.73

39.33

32.00

35.62

13.66

42.69

$-3.79$

40.64

31.67

30.31

52.44

93.46
Mean Growth Percent - Growth Percent

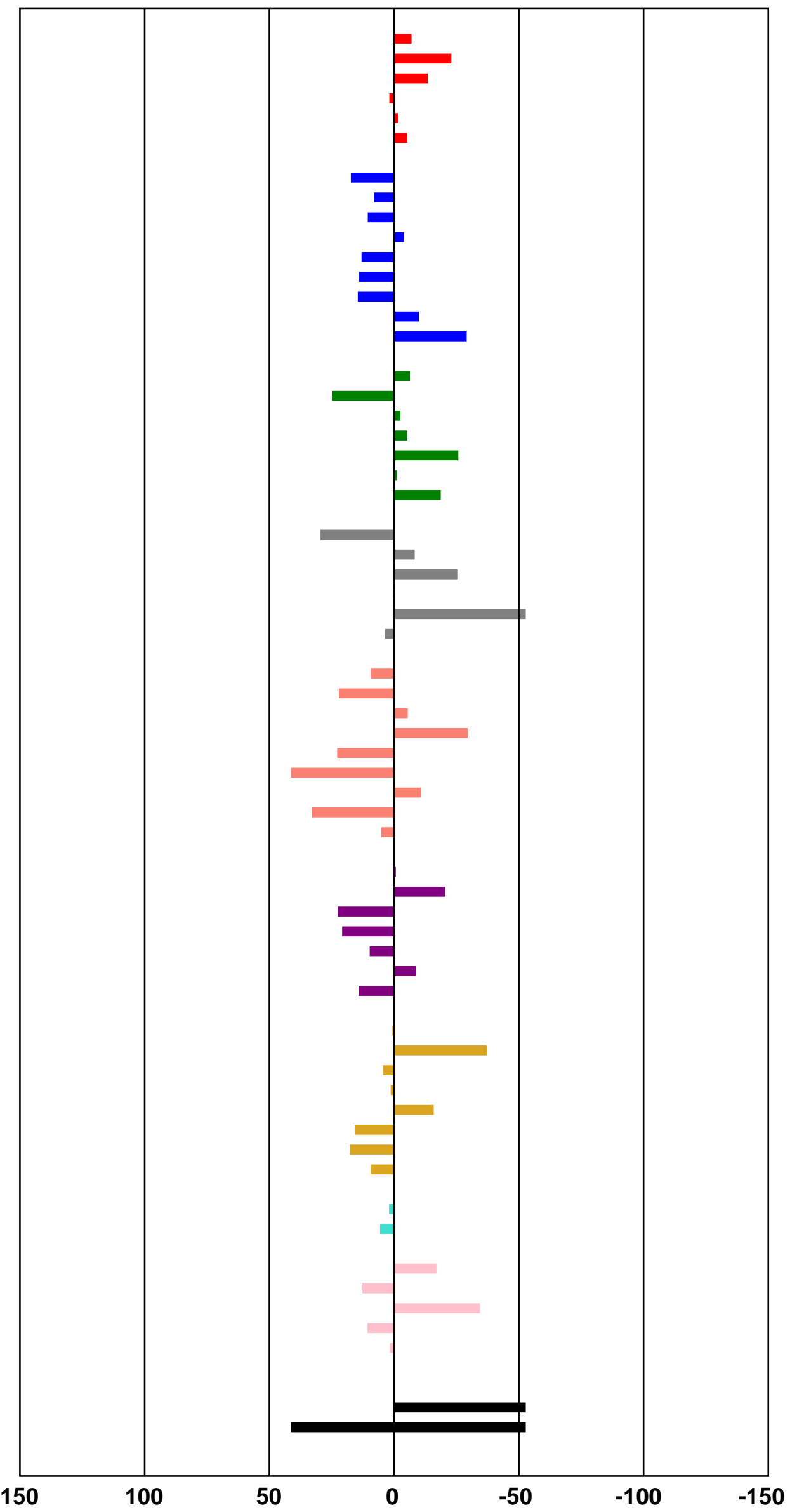


Developmental Therapeutics Program One Dose Mean Graph
NSC: D-824742 / 1

Experiment ID: $20080 S 41$

\section{Panel/Cell Line}

Leukemia

CCRF-CEM

RPMI-8226

SR

Non-Small Cell Lung Cancer A549/ATCC

EKVX

HOP-62

HOP-92

$\mathrm{NCl}-\mathrm{H} 226$

$\mathrm{NCl}-\mathrm{H} 23$

$\mathrm{NCl}-\mathrm{H} 322 \mathrm{M}$

$\mathrm{NCl}-\mathrm{H} 460$

$\mathrm{NCl}-\mathrm{H} 522$

Colon Cancer

COLO 205

HCC-2998

HCT-116

HCT-15

HT29

KM12

SW-620

CNS Cancer

SF-268

SF-295

SF-539

SNB-19

SNB-75

U251

Melanoma

LOX IMVI

MALME-3M

M14

MDA-MB-435

SK-MEL-2

SK-MEL-28

SK-MEL-5

UACC-257

UACC-62

Ovarian Cancer

IGROV1

OVCAR-3

OVCAR-4

OVCAR-5

OVCAR-8

NCI/ADR-RES

SK-OV-3

Renal Cancer

786-0

A498

ACHN

CAKI-1

RXF 393

SN12C

TK-10

UO-31

Prostate Cancer

PC-3

DU-145

Breast Cancer

MCF7

MDA-MB-231/ATCC

HS 578T

BT-549

T-47D

Mean
Delta

Range

\section{Growth Percent}

56.85

55.21

28.56

55.20

75.25

60.74

83.28

77.91

72.84

88.73

55.79

39.93

67.52

84.14

41.09

35.18

28.52

38.65

37.17

77.46

43.63

65.52

60.96

46.56

68.46

55.87

65.82

48.63

$-4.02$

84.79

64.05

49.02

73.74

47.30

51.83

24.47

69.38

93.24

78.45

43.26

86.84

85.55

47.02

76.43

42.72

69.39

88.63

93.70

57.96

68.15

87.11

26.60

78.79

68.96

63.78

55.13

60.49

64.51

97.72
Mean Growth Percent - Growth Percent

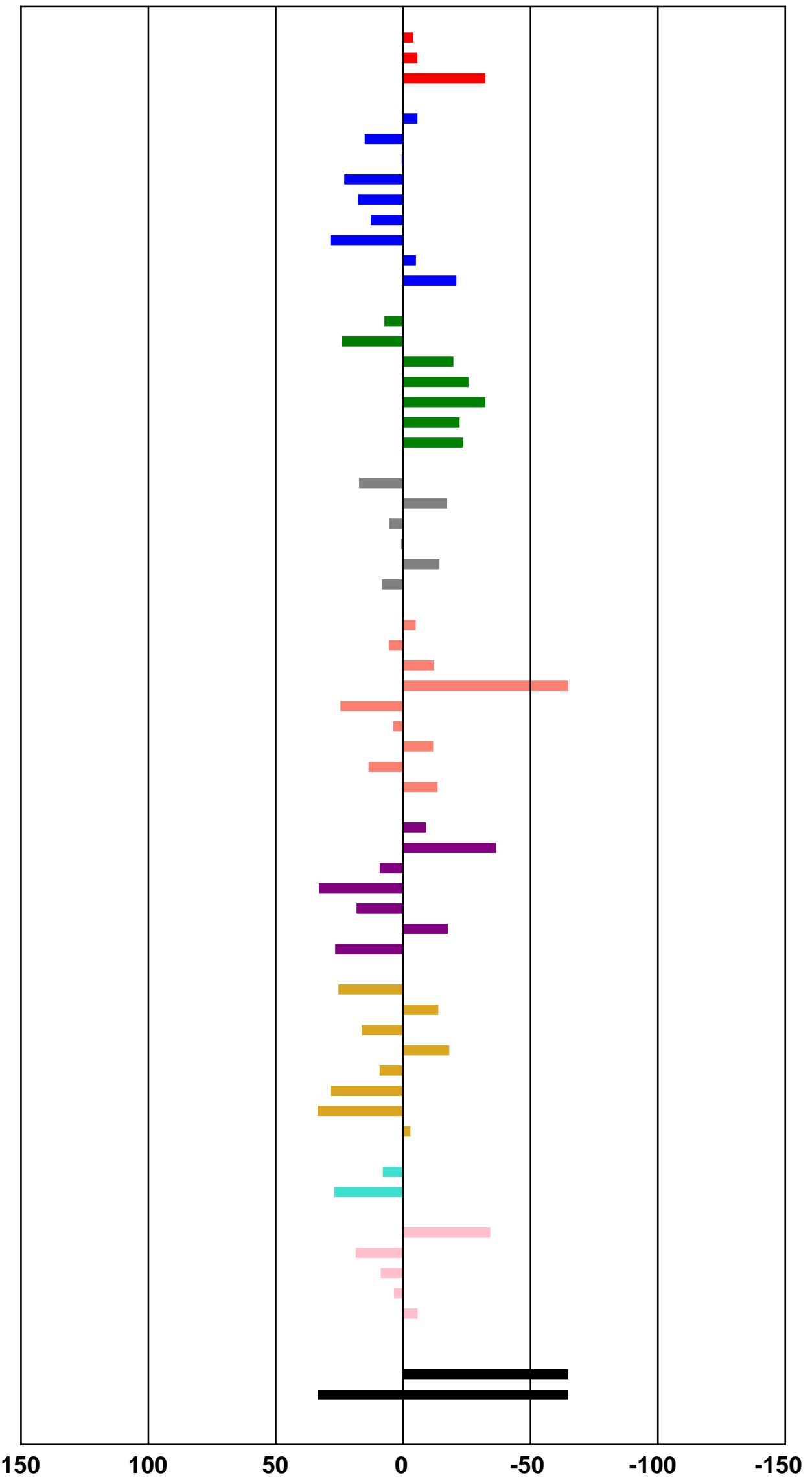


Developmental Therapeutics Program One Dose Mean Graph
NSC: D-823376 / 1

Experiment ID: $20020 S 20$
Test Date: Feb 24, 2020

Report Date: Mar 24, 2020

\section{Panel/Cell Line}

Leukemia

CCRF-CEM

HL-60(TB)

$\mathrm{K}-562$

MOLT-4

RPMI-8226

Non-Small Cell Lung Cancer

A549/ATCC

EKVX

HOP-62

HOP-92

$\mathrm{NCl}-\mathrm{H} 226$

$\mathrm{NCl}-\mathrm{H} 23$

$\mathrm{NCl}-\mathrm{H} 322 \mathrm{M}$

$\mathrm{NCl}-\mathrm{H} 460$

$\mathrm{NCl}-\mathrm{H} 522$

Colon Cancer

COLO 205

HCC-2998

HCT-116

HCT-15

HT29

KM12

SW-620

CNS Cancer

SF-268

SF-295

SF-539

SNB-19

SNB-75

Melanoma

LOX IMVI

MALME-3M

M14

MDA-MB-435

SK-MEL-2

SK-MEL-28

SK-MEL-5

UACC-257

UACC-62

Ovarian Cancer

IGROV1

OVCAR-3

OVCAR-4

OVCAR-5

OVCAR-8

NCI/ADR-RES

SK-OV-3

Renal Cancer

786-0

A498

ACHN

CAKI-1

RXF 393

SN12C

TK-10

UO-31

Prostate Cancer

PC-3

DU-145

Breast Cancer MCF7

MDA-MB-231/ATCC

HS 578T

BT-549

T-47D

MDA-MB-468

\section{Growth Percent}

108.57

116.49

64.71

92.57

97.92

96.63

89.82

91.44

83.30

90.82

90.09

93.67

100.20

81.95

106.18

104.17

93.89

78.34

106.62

98.97

86.54

93.78

95.90

98.44

86.26

59.50

97.21

82.78

91.63

51.93

102.94

97.75

94.58

93.88

75.60

88.50

110.86

89.28

95.48

96.02

86.26

108.18

95.66

83.30

102.90

76.13

101.02

96.37

115.99

83.17

92.90

101.69

89.30

90.54

88.68

96.30

89.72

72.64

92.17

40.24

64.56
Mean Growth Percent - Growth Percent

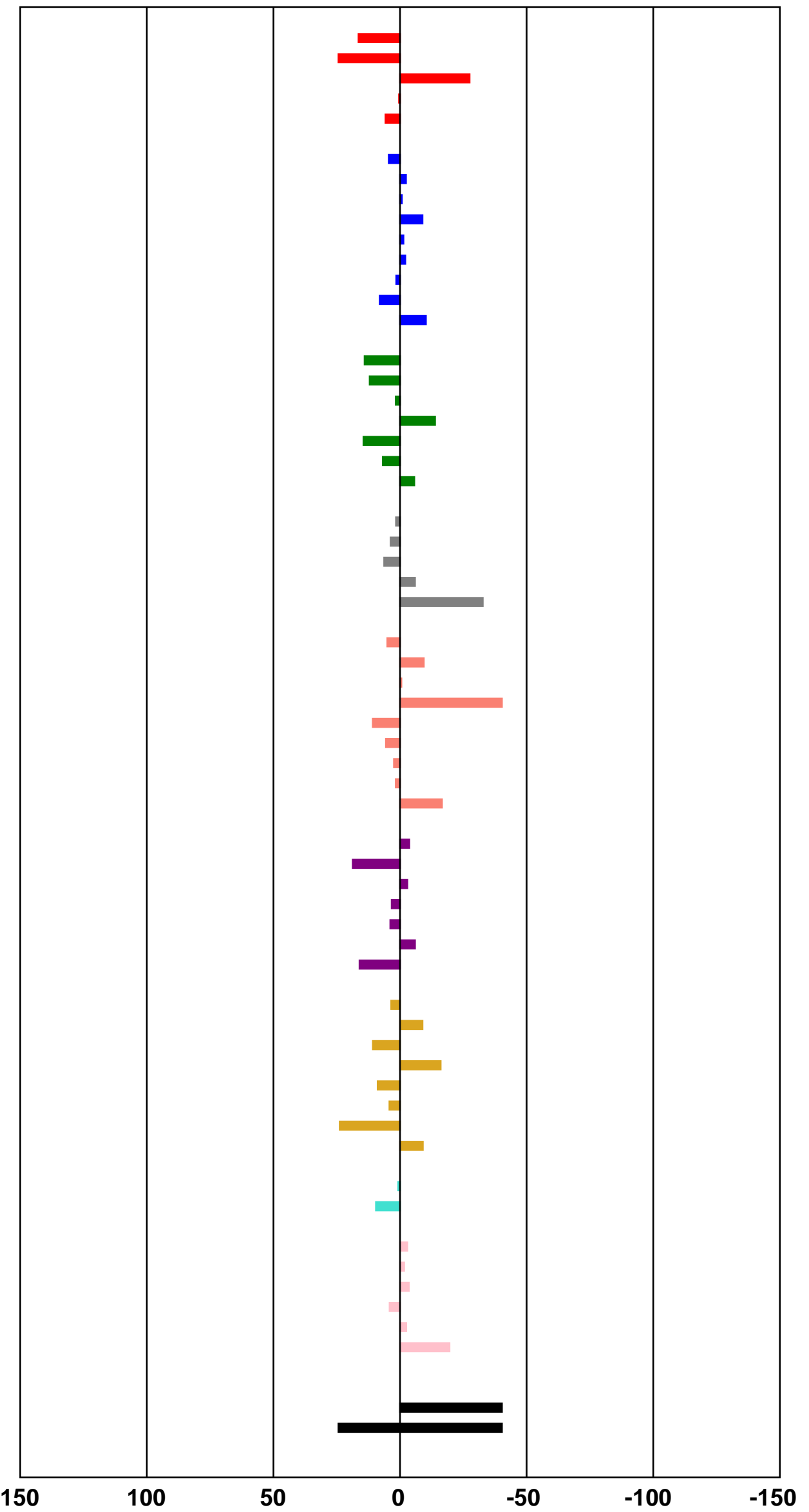


Developmental Therapeutics Program One Dose Mean Graph
NSC: D-824743/1

Conc: $1.00 \mathrm{E}-5$ Molar

Experiment ID: $20080 S 41$

\section{Panel/Cell Line}

Leukemia

CCRF-CEM

HL-60(TB)

$\mathrm{K}-562$

MOLT-4

RPMI-8226

Non-Small Cell Lung Cancer

A549/ATCC

EKVX

HOP-62

HOP-92

$\mathrm{NCl}-\mathrm{H} 226$

$\mathrm{NCl}-\mathrm{H} 23$

$\mathrm{NCl}-\mathrm{H} 322 \mathrm{M}$

$\mathrm{NCl}-\mathrm{H} 460$

$\mathrm{NCl}-\mathrm{H} 522$

Colon Cancer

COLO 205

HCC-2998

HCT-116

HCT-15

HT29

KM12

SW-620

CNS Cancer

SF-268

SF-295

SF-539

SNB-19

SNB-75

U251

Melanoma

LOX IMVI

MALME-3M

M14

MDA-MB-435

SK-MEL-2

SK-MEL-28

SK-MEL-5

UACC-257

UACC-62

Ovarian Cancer

IGROV1

OVCAR-3

OVCAR-4

OVCAR-5

OVCAR-8

NCI/ADR-RES

SK-OV-3

Renal Cancer

786-0

A498

ACHN

CAKI-1

RXF 393

SN12C

TK-10

UO-31

Prostate Cancer

PC-3

DU-145

Breast Cancer

MCF7

MDA-MB-231/ATCC

HS 578T

BT -549

T-47D

\section{Growth Percent}

22.02

$-36.00$

9.89

2.78

19.27

28.43

32.39

48.54

58.32

27.70

31.06

50.68

12.18

13.53

$-10.95$

15.92

12.94

14.14

5.04

15.53

21.95

47.01

16.94

$-19.09$

22.79

$-2.31$

24.79

22.40

67.97

10.21

$-36.37$

80.36

49.10

20.37

69.40

26.74

23.27

$-16.88$

39.98

27.76

27.40

6.12

50.80

28.59

$-5.20$

27.49

43.36

16.23

46.68

66.88

45.80

26.63

21.03

12.68

26.20

22.13

11.53

49.61

24.03

60.40

116.73
Mean Growth Percent - Growth Percent

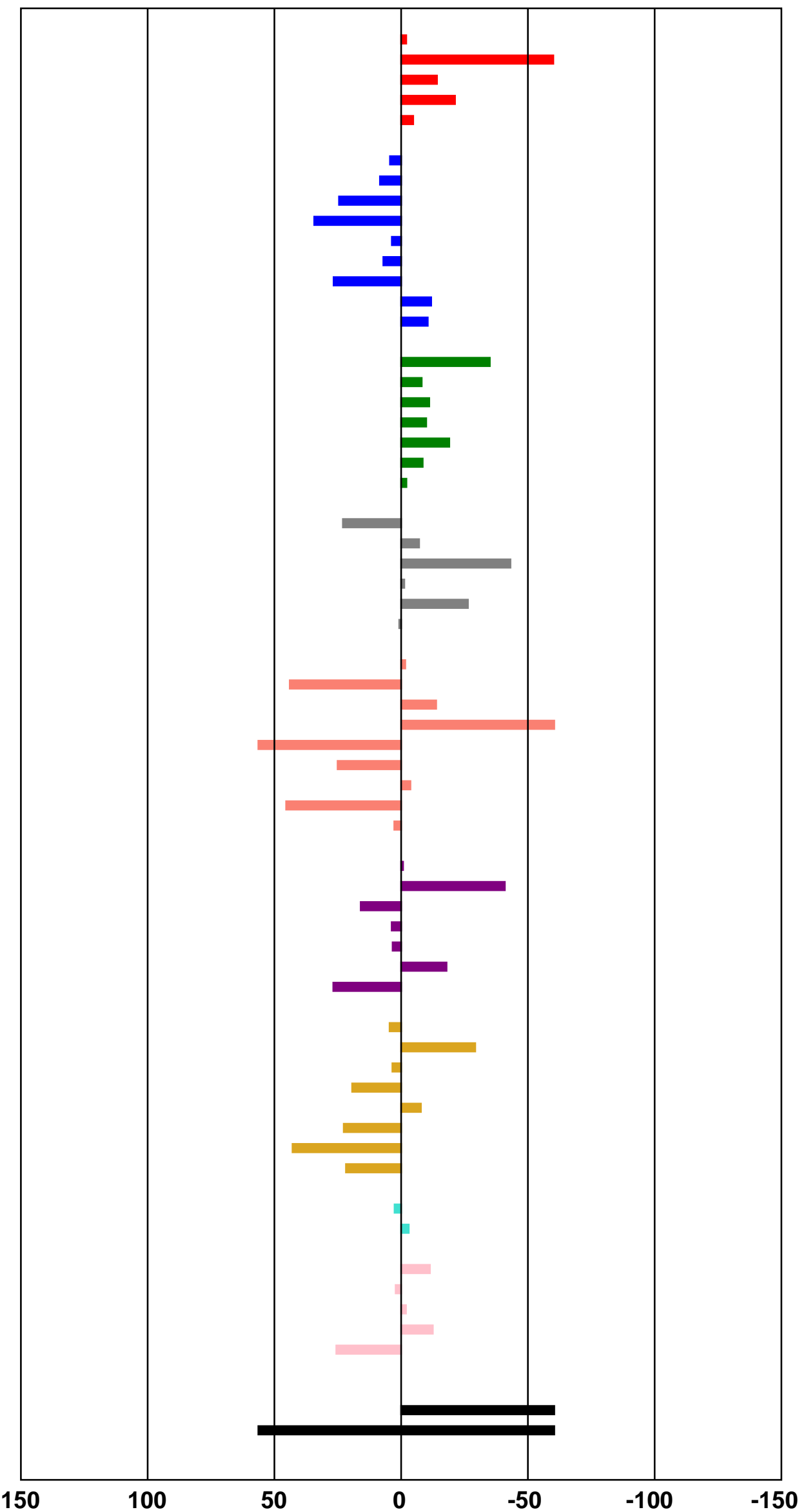


Developmental Therapeutics Program One Dose Mean Graph
NSC: D-824744 / 1

Experiment ID: $20080 S 41$
Test Date: Aug 24, 2020

Report Date: Sep 15, 2020

\section{Panel/Cell Line}

Leukemia

CCRF-CEM

HL-60(TB)

K-562

RPMI-8226

SR

Non-Small Cell Lung Cancer

A549/ATCC

EKVX

HOP-62

HOP-92

$\mathrm{NCl}-\mathrm{H} 226$

$\mathrm{NCl}-\mathrm{H} 23$

$\mathrm{NCl}-\mathrm{H} 322 \mathrm{M}$

$\mathrm{NCl}-\mathrm{H} 460$

$\mathrm{NCl}-\mathrm{H} 522$

Colon Cancer

COLO 205

HCC-2998

HCT-116

HCT-15

HT29

KM12

SW-620

CNS Cancer

SF-268

SF-295

SF-539

SNB-19

SNB-75

U251

Melanoma

LOX IMVI

MALME-3M

M14

MDA-MB-435

SK-MEL-2

SK-MEL-28

SK-MEL-5

UACC-257

UACC-62

Ovarian Cancer

IGROV1

OVCAR-3

OVCAR-4

OVCAR-5

OVCAR-8

NCI/ADR-RES

SK-OV-3

Renal Cancer

786-0

A498

$\mathrm{ACHN}$

CAKI-1

RXF 393

SN12C

TK-10

UO-31

Prostate Cancer

PC-3

DU-145

Breast Cancer

MCF7

MDA-MB-231/ATCC

HS 578T

BT -549

T-47D

\section{Growth Percent}

14.10

$-22.02$

8.53
18.80

15.67

26.52

31.36

42.74

73.91

33.76

36.24

44.43

14.33

3.84

$-0.41$

26.82

13.81

16.94

3.93

16.55

18.57

56.21

16.07

$-8.06$

18.90

$-10.15$

24.11

33.01

62.41

2.07

$-59.64$

56.86

53.74

26.37

67.80

26.48

26.95

$-1.26$

48.30

26.43

28.07

19.46

44.53

27.79

$-5.72$

36.03

36.63

9.98

60.67

68.47

44.26

31.13

21.59

12.43

32.48

13.49

34.48

44.95

25.27

84.91

133.55
Mean Growth Percent - Growth Percent

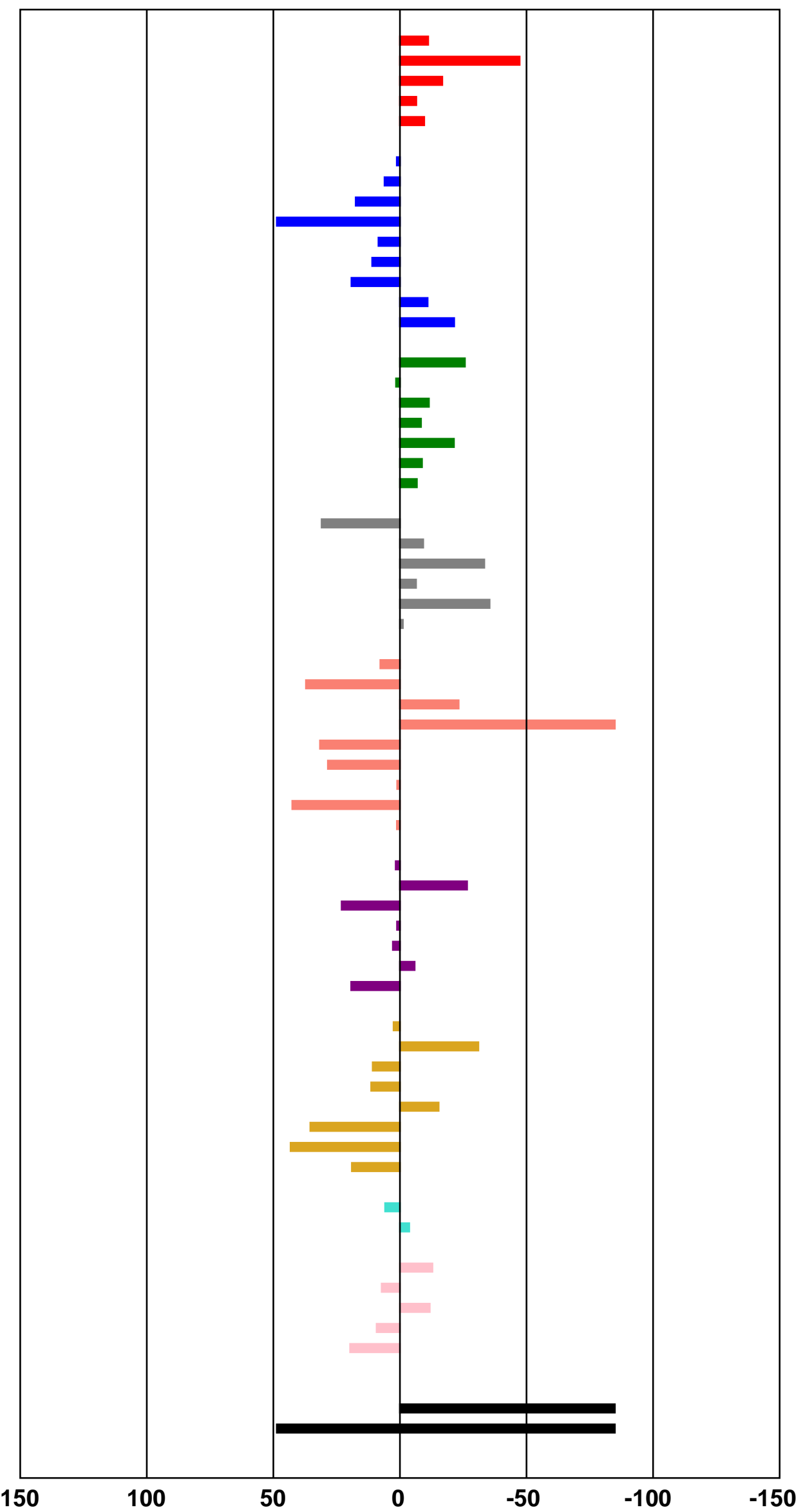




\section{Panel/Cell Line}

Leukemia

CCRF-CEM

HL-60(TB)

K-562

MOLT-4

$\mathrm{SR}$

Non-Small Cell Lung Cancer

A549/ATCC

EKVX

HOP-62

HOP-92

$\mathrm{NCl}-\mathrm{H} 226$

$\mathrm{NCl}-\mathrm{H} 23$

$\mathrm{NCl}-\mathrm{H} 322 \mathrm{M}$

$\mathrm{NCl}-\mathrm{H} 460$

$\mathrm{NCl}-\mathrm{H} 522$

Colon Cancer

COLO 205

HCC-2998

HCT-116

HCT-15

HT29

KM12

SW-620

CNS Cancer

SF-268

SF-295

SF-539

SNB-19

SNB-75

U251

Melanoma

LOX IMVI

MALME-3M

M14

SK-MEL-2

SK-MEL-28

SK-MEL-5

UACC-257

UACC-62

Ovarian Cancer

IGROV1

OVCAR-3

OVCAR-4

OVCAR-5

OVCAR-8

NCI/ADR-RES

SK-OV-3

Renal Cancer

786-0

A498

ACHN

CAKI-1

RXF 393

SN12C

TK-10

UO-31

Prostate Cancer

PC-3

DU-145

Breast Cancer

MCF7

MDA-MB-231/ATCC

HS 578T

BT-549

T-47D

MDA-MB-468

\section{Growth Percent}

52.29

34.48

17.66

41.90

39.57

57.13

73.77

56.16

66.33

75.07

69.87

92.11

42.03

50.11

63.51

79.31

38.88

36.82

27.28

49.11

28.44

76.69

42.79

60.65

68.70

36.57

56.62

53.04

58.54

45.69

54.96

70.35

67.42

74.90

40.24

53.42

37.08

71.68

86.17

71.34

43.39

79.88

76.67

51.79

82.59

22.11

62.09

70.86

96.57

72.82

61.41

83.34

29.94

68.43

67.26

68.63

44.89

19.89

57.26

39.60

78.91
Mean Growth Percent - Growth Percent

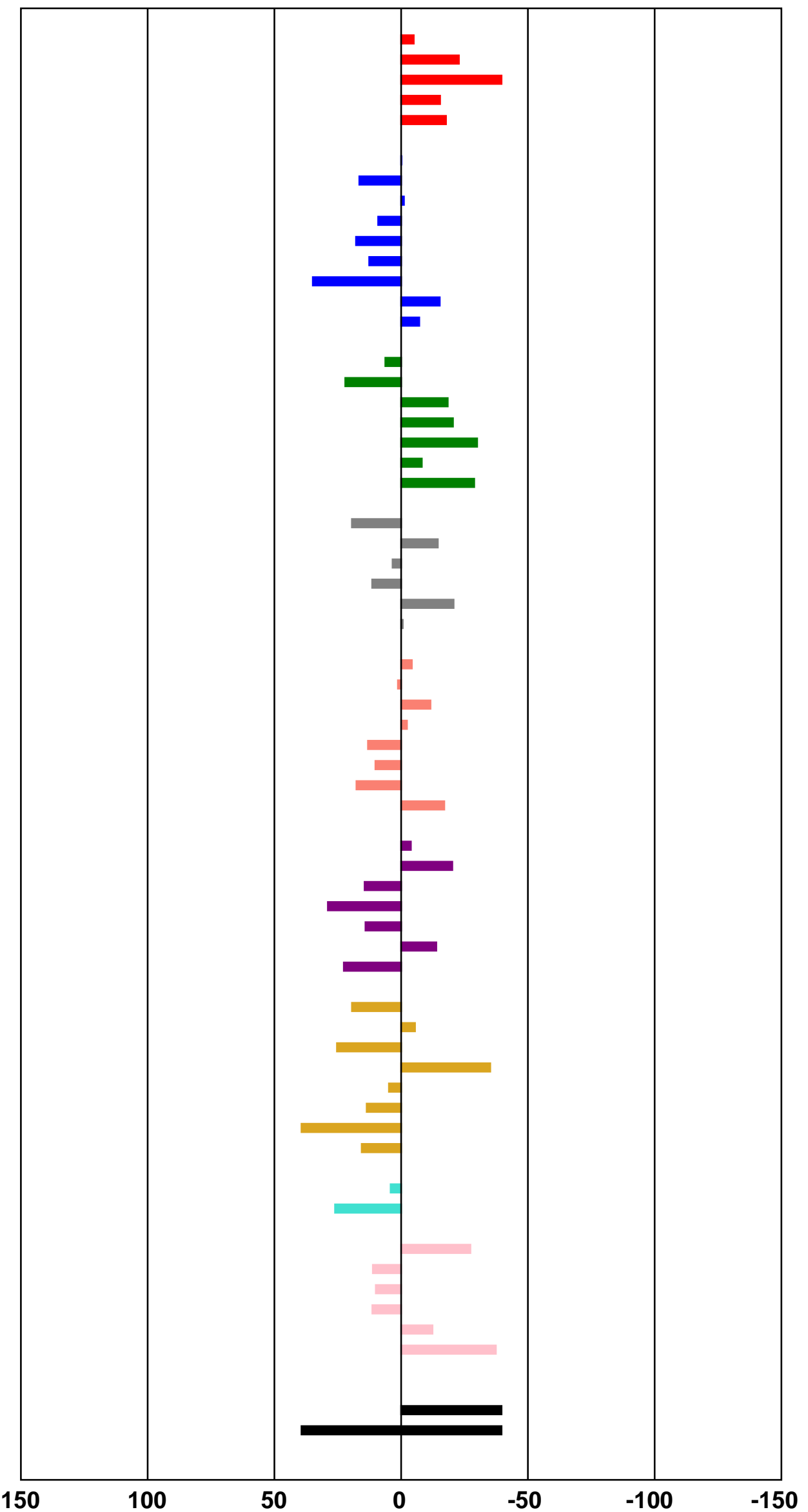


One Dose Mean Graph

\section{Panel/Cell Line}

Leukemia

CCRF-CEM

HL-60(TB)

K-562

MOLT-4

$\mathrm{SR}$

Non-Small Cell Lung Cancer

A549/ATCC

EKVX

HOP-62

HOP-92

$\mathrm{NCl}-\mathrm{H} 226$

$\mathrm{NCl}-\mathrm{H} 23$

$\mathrm{NCl}-\mathrm{H} 322 \mathrm{M}$

$\mathrm{NCl}-\mathrm{H} 460$

$\mathrm{NCl}-\mathrm{H} 522$

Colon Cancer

COLO 205

HCC-2998

HCT-116

HCT-15

HT29

KM12

SW-620

CNS Cancer

SF-268

SF-295

SF-539

SNB-19

SNB-75

U251

Melanoma

LOX IMVI

MALME-3M

M14

SK-MEL-2

SK-MEL-28

SK-MEL-5

UACC-257

UACC-62

Ovarian Cancer

IGROV1

OVCAR-3

OVCAR-4

OVCAR-5

OVCAR-8

NCI/ADR-RES

SK-OV-3

Renal Cancer

786-0

A498

ACHN

CAKI-1

RXF 393

SN12C

TK-10

UO-31

Prostate Cancer

PC-3

DU-145

Breast Cancer

MCF7

MDA-MB-231/ATCC

HS $578 \mathrm{~T}$

BT-549

T-47D

MDA-MB-468

\section{Growth Percent}

91.83

92.71

83.48

92.36

101.24

95.96

94.74

94.33

82.13

91.25

93.02

94.10

103.11

85.96

108.73

108.28

94.23

96.03

100.95

96.82

98.85

95.96

91.26

99.01

95.09

92.54

96.92

96.15

93.15

99.46

103.22

108.41

98.92

104.28

89.95

89.03

102.48

92.87

100.83

98.23

96.61

110.17

98.53

99.89

99.63

97.72

103.75

91.52

109.39

85.75

88.27

102.77

91.08

93.55

102.87

96.01

103.12

91.10

96.72

14.59

28.04
Mean Growth Percent - Growth Percent

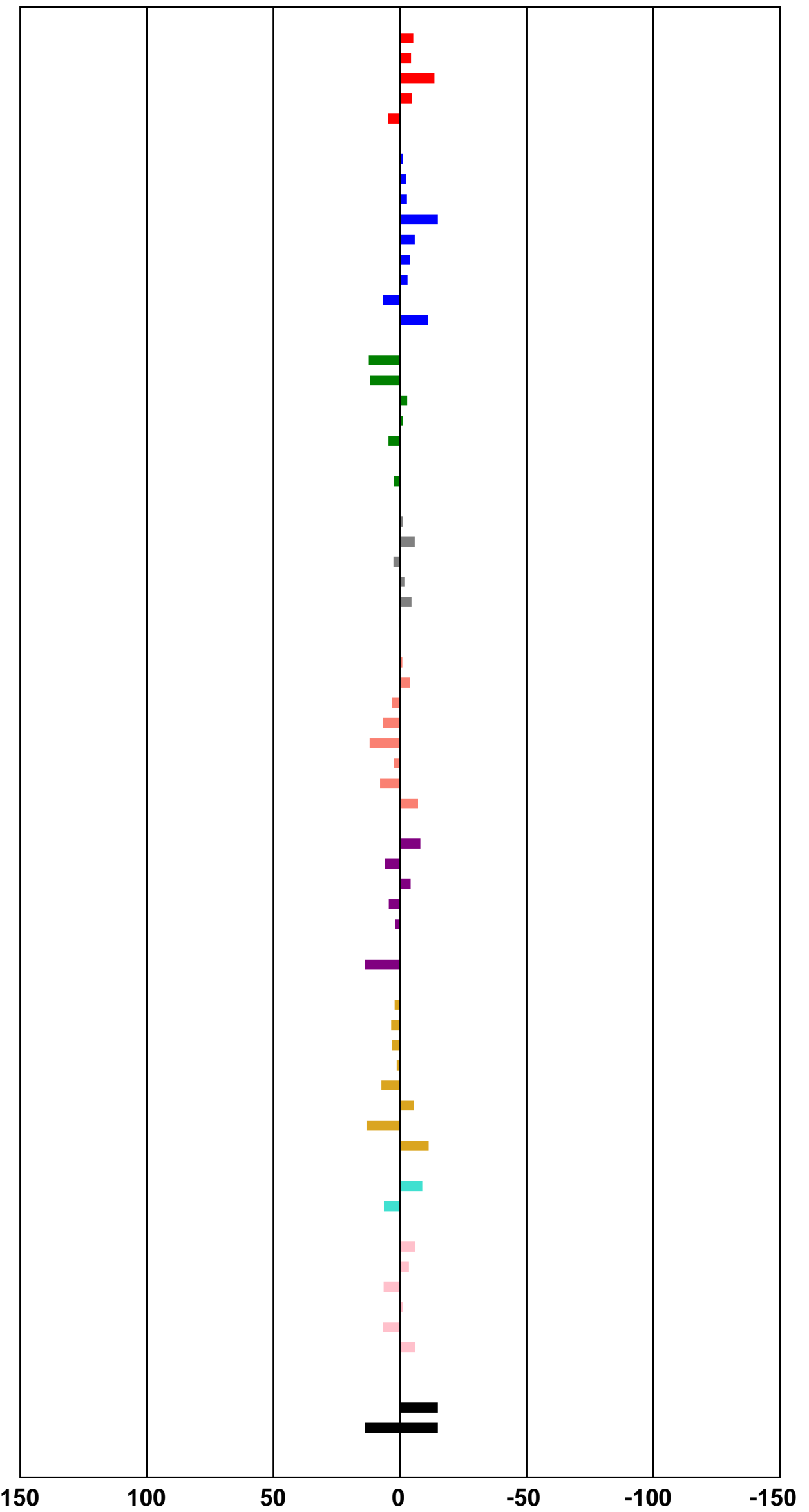




\section{Panel/Cell Line}

Leukemia

CCRF-CEM

HL-60(TB)

K-562

MOLT-4

$\mathrm{SR}$

Non-Small Cell Lung Cancer

A549/ATCC

EKVX

HOP-62

HOP-92

$\mathrm{NCl}-\mathrm{H} 226$

$\mathrm{NCl}-\mathrm{H} 23$

$\mathrm{NCl}-\mathrm{H} 322 \mathrm{M}$

$\mathrm{NCl}-\mathrm{H} 460$

$\mathrm{NCl}-\mathrm{H} 522$

Colon Cancer

COLO 205

HCC-2998

HCT-116

HCT-15

HT29

KM12

SW-620

CNS Cancer

SF-268

SF-295

SF-539

SNB-19

SNB-75

U251

Melanoma

LOX IMVI

MALME-3M

M14

SK-MEL-2

SK-MEL-28

SK-MEL-5

UACC-257

UACC-62

Ovarian Cancer

IGROV1

OVCAR-3

OVCAR-4

OVCAR-5

OVCAR-8

NCI/ADR-RES

SK-OV-3

Renal Cancer

786-0

A498

ACHN

CAKI-1

RXF 393

SN12C

TK-10

UO-31

Prostate Cancer

PC-3

DU-145

Breast Cancer

MCF7

MDA-MB-231/ATCC

HS 578T

BT-549

T-47D

MDA-MB-468

\section{Growth Percent}

57.91

38.91

15.74

41.27

31.06

62.17

78.38

62.19

77.29

74.86

73.91

92.34

73.21

59.64

88.89

91.07

48.54

39.94

38.35

48.33

33.84

78.00

49.41

62.86

73.67

53.85

68.70

55.70

66.62

53.55

64.80

73.23

74.68

79.83

48.53

58.15

53.19

67.09

94.25

80.18

47.67

87.01

88.50

59.96

87.60

27.53

74.57

75.00

100.88

71.33

63.06

88.51

33.33

72.51

69.00

76.34

51.47

18.39

63.39

47.65

85.14
Mean Growth Percent - Growth Percent

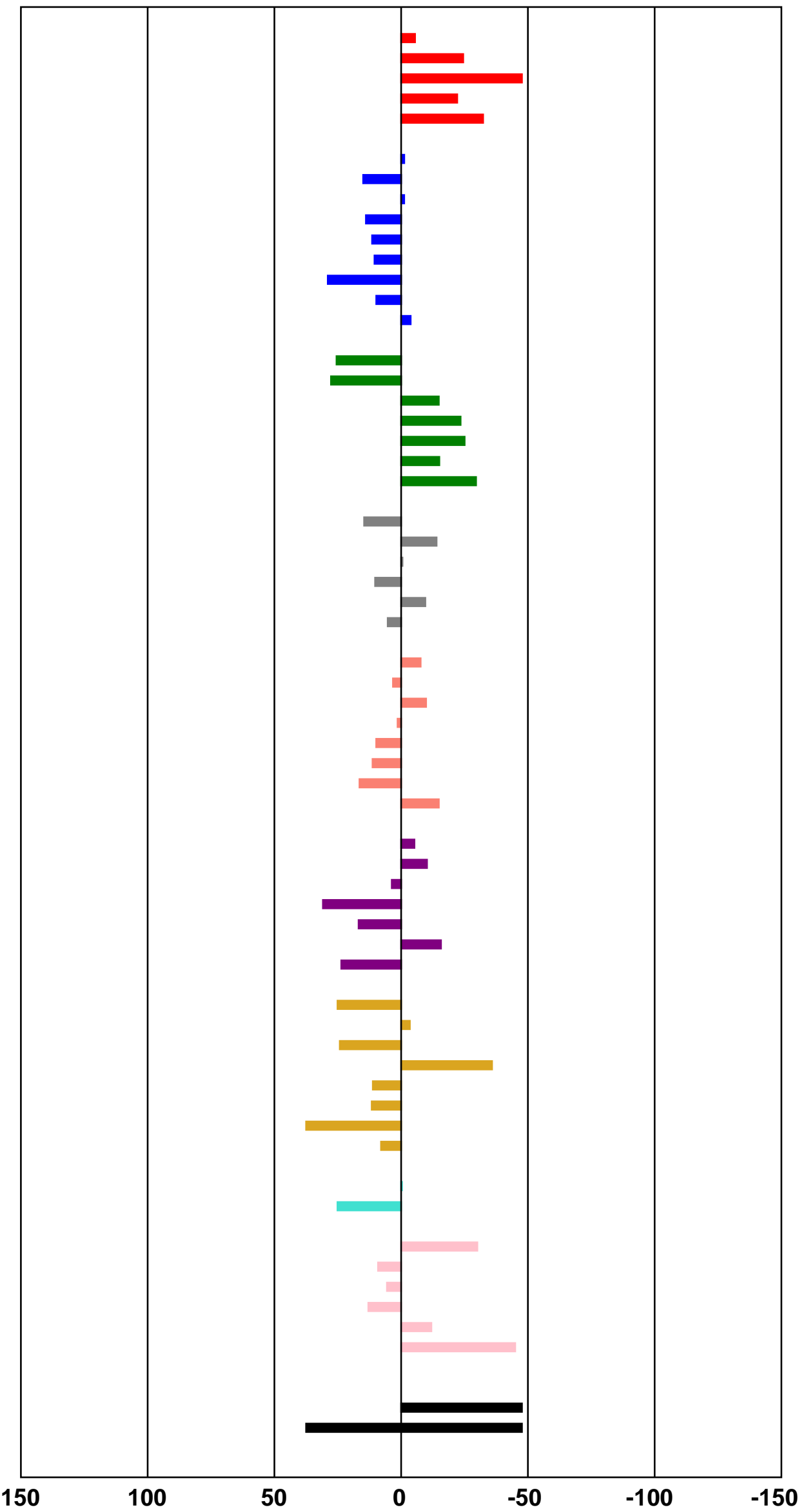


NSC: D-826089/1

\section{Panel/Cell Line}

Leukemia

CCRF-CEM

HL-60(TB)

K-562

MOLT-4

$\mathrm{SR}$

Non-Small Cell Lung Cancer

A549/ATCC

EKVX

HOP-62

HOP-92

$\mathrm{NCl}-\mathrm{H} 226$

$\mathrm{NCl}-\mathrm{H} 23$

$\mathrm{NCl}-\mathrm{H} 322 \mathrm{M}$

$\mathrm{NCl}-\mathrm{H} 460$

$\mathrm{NCl}-\mathrm{H} 522$

Colon Cancer

COLO 205

HCC-2998

HCT-116

HCT-15

HT29

KM12

SW-620

CNS Cancer

SF-268

SF-295

SF-539

SNB-19

SNB-75

U251

Melanoma

LOX IMVI

MALME-3M

M14

SK-MEL-2

SK-MEL-28

SK-MEL-5

UACC-257

UACC-62

Ovarian Cancer

IGROV1

OVCAR-3

OVCAR-4

OVCAR-5

OVCAR-8

NCl/ADR-RES

SK-OV-3

Renal Cancer

786-0

A498

ACHN

CAKI-1

RXF 393

SN12C

TK-10

UO-31

Prostate Cancer

PC-3

DU-145

Breast Cancer

MCF7

MDA-MB-231/ATCC

HS 578T

BT-549

T-47D

MDA-MB-468

\section{Growth Percent}

17.24

3.27

10.06

32.44

23.23

46.15

38.17

30.21

55.44

35.53

37.26

52.22

18.30

33.20

12.43

53.15

26.69

29.35

10.57

22.73

22.55

66.07

10.55

13.33

43.85

$-2.75$

36.04

39.53

50.04

21.13

49.91

66.91

18.09

66.91

34.06

41.39

15.46

52.54

48.80

40.38

18.64

60.05

46.74

13.11

46.10

$-22.90$

0.10

50.45

68.98

51.76

25.18

24.08

17.35

41.92

28.06

57.51

30.29

9.75

32.58

55.48

91.88
Mean Growth Percent - Growth Percent

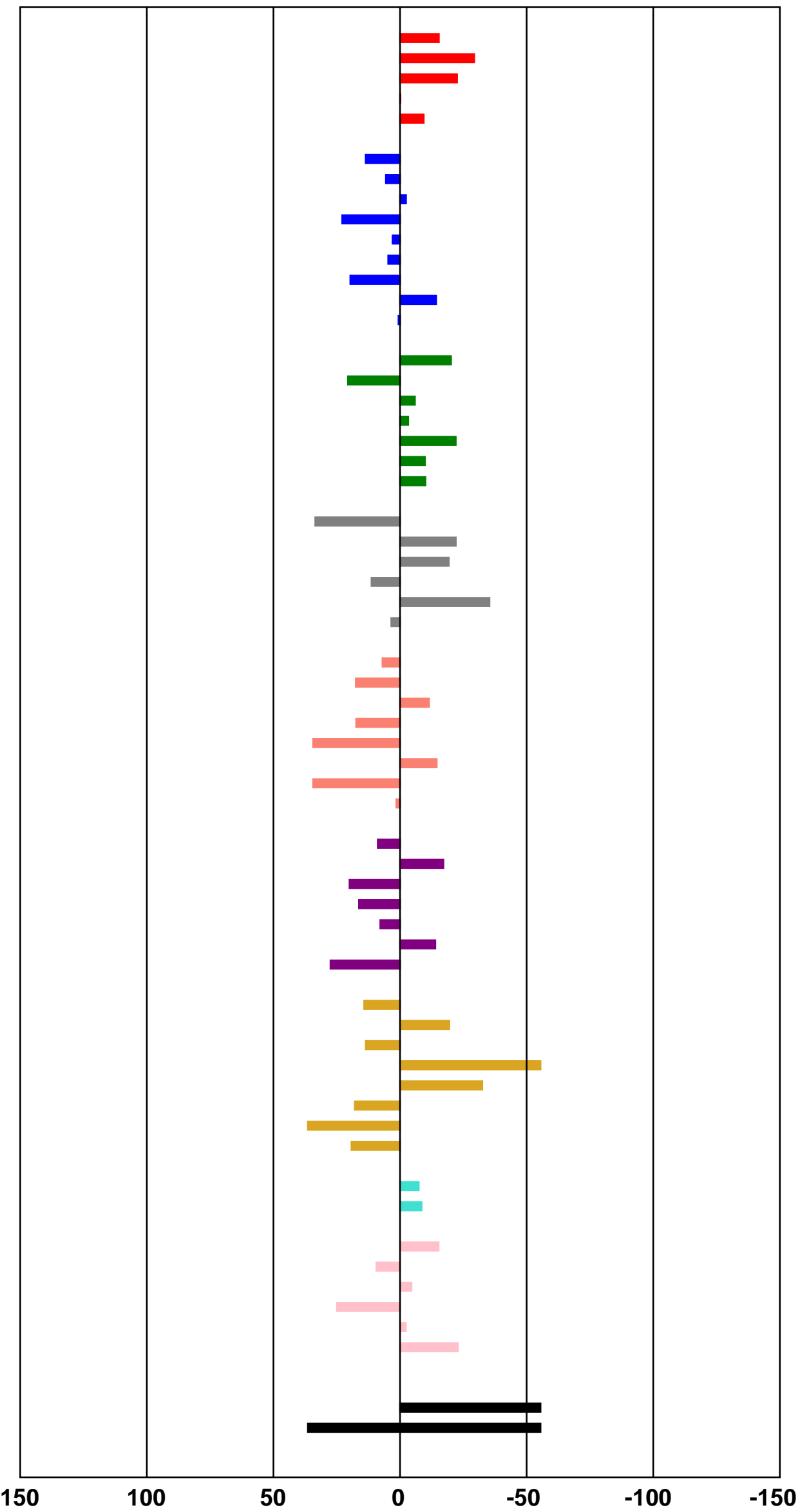




\section{Panel/Cell Line}

Leukemia

CCRF-CEM

HL-60(TB)

K-562

MOLT-4

$\mathrm{SR}$

Non-Small Cell Lung Cancer

A549/ATCC

EKVX

HOP-62

HOP-92

$\mathrm{NCl}-\mathrm{H} 226$

$\mathrm{NCl}-\mathrm{H} 23$

$\mathrm{NCl}-\mathrm{H} 322 \mathrm{M}$

$\mathrm{NCl}-\mathrm{H} 460$

$\mathrm{NCl}-\mathrm{H} 522$

Colon Cancer

COLO 205

HCC-2998

HCT-116

HCT-15

HT29

KM12

SW-620

CNS Cancer

SF-268

SF-295

SF-539

SNB-19

SNB-75

U251

Melanoma

LOX IMVI

MALME-3M

M14

SK-MEL-2

SK-MEL-28

SK-MEL-5

UACC-257

UACC-62

Ovarian Cancer

IGROV1

OVCAR-3

OVCAR-4

OVCAR-5

OVCAR-8

NCI/ADR-RES

SK-OV-3

Renal Cancer

786-0

A498

ACHN

CAKI-1

RXF 393

SN12C

TK-10

UO-31

Prostate Cancer

PC-3

DU-145

Breast Cancer

MCF7

MDA-MB-231/ATCC

HS 578T

BT-549

T-47D

MDA-MB-468

\section{Growth Percent}

14.06

$-1.60$

9.83

29.23

27.89

50.29

42.46

29.04

48.99

41.90

33.78

45.96

12.55

24.74

11.96

59.12

26.61

27.81

8.12

31.98

15.28

59.42

12.16

10.91

42.75

$-12.98$

31.05

36.15

49.44

20.85

43.68

59.69

16.84

69.92

31.21

36.35

$-9.98$

56.81

45.24

38.88

14.61

50.18

41.71

2.08

41.54

6.99

2.46

42.88

53.34

51.67

20.45

19.55

16.97

44.85

17.85

57.14

26.90

$-9.49$

29.83

42.81

82.90
Mean Growth Percent - Growth Percent

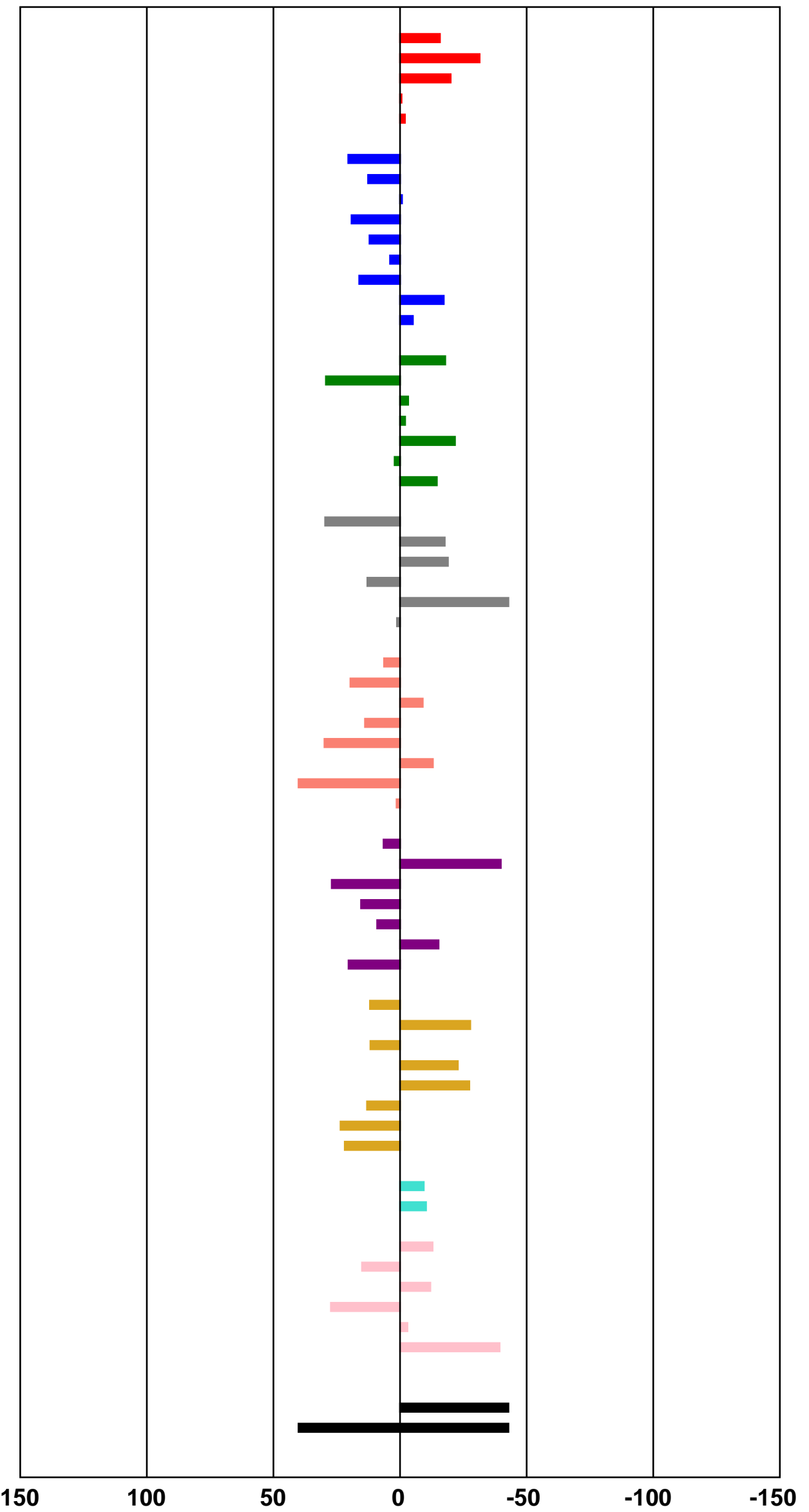




\section{Panel/Cell Line}

Leukemia

CCRF-CEM

HL-60(TB)

K-562

MOLT-4

SR

Non-Small Cell Lung Cancer

A549/ATCC

EKVX

HOP-62

HOP-92

$\mathrm{NCl}-\mathrm{H} 226$

$\mathrm{NCl}-\mathrm{H} 23$

$\mathrm{NCl}-\mathrm{H} 322 \mathrm{M}$

$\mathrm{NCl}-\mathrm{H} 460$

$\mathrm{NCl}-\mathrm{H} 522$

Colon Cancer

COLO 205

HCC-2998

HCT-116

HCT-15

HT29

KM12

SW-620

CNS Cancer

SF-268

SF-295

SF-539

SNB-19

SNB-75

U251

Melanoma

LOX IMVI

MALME-3M

M14

SK-MEL-2

SK-MEL-28

SK-MEL-5

UACC-257

UACC-62

Ovarian Cancer

IGROV1

OVCAR-3

OVCAR-4

OVCAR-5

OVCAR-8

NCI/ADR-RES

SK-OV-3

Renal Cancer

786-0

A498

ACHN

CAKI-1

RXF 393

SN12C

TK-10

UO-31

Prostate Cancer

PC-3

DU-145

Breast Cancer

MCF7

MDA-MB-231/ATCC

HS 578T

BT-549

T-47D

MDA-MB-468

\section{Growth Percent}

24.13

3.85

9.69

30.33

27.81

47.71

58.01

35.93

66.75

60.76

55.21

83.33

17.27

43.19

47.37

72.80

23.72

26.17

17.20

30.90

23.71

60.62

22.57

37.84

46.96

18.98

32.64

37.67

50.23

25.38

41.26

58.28

38.05

58.91

29.43

42.77

20.26

59.45

73.22

56.32

24.74

65.57

47.23

23.22

57.15

17.53

49.54

52.82

84.35

56.69

45.26

68.57

19.91

48.12

40.61

52.14

31.62

15.32

41.67

37.82

80.50
Mean Growth Percent - Growth Percent

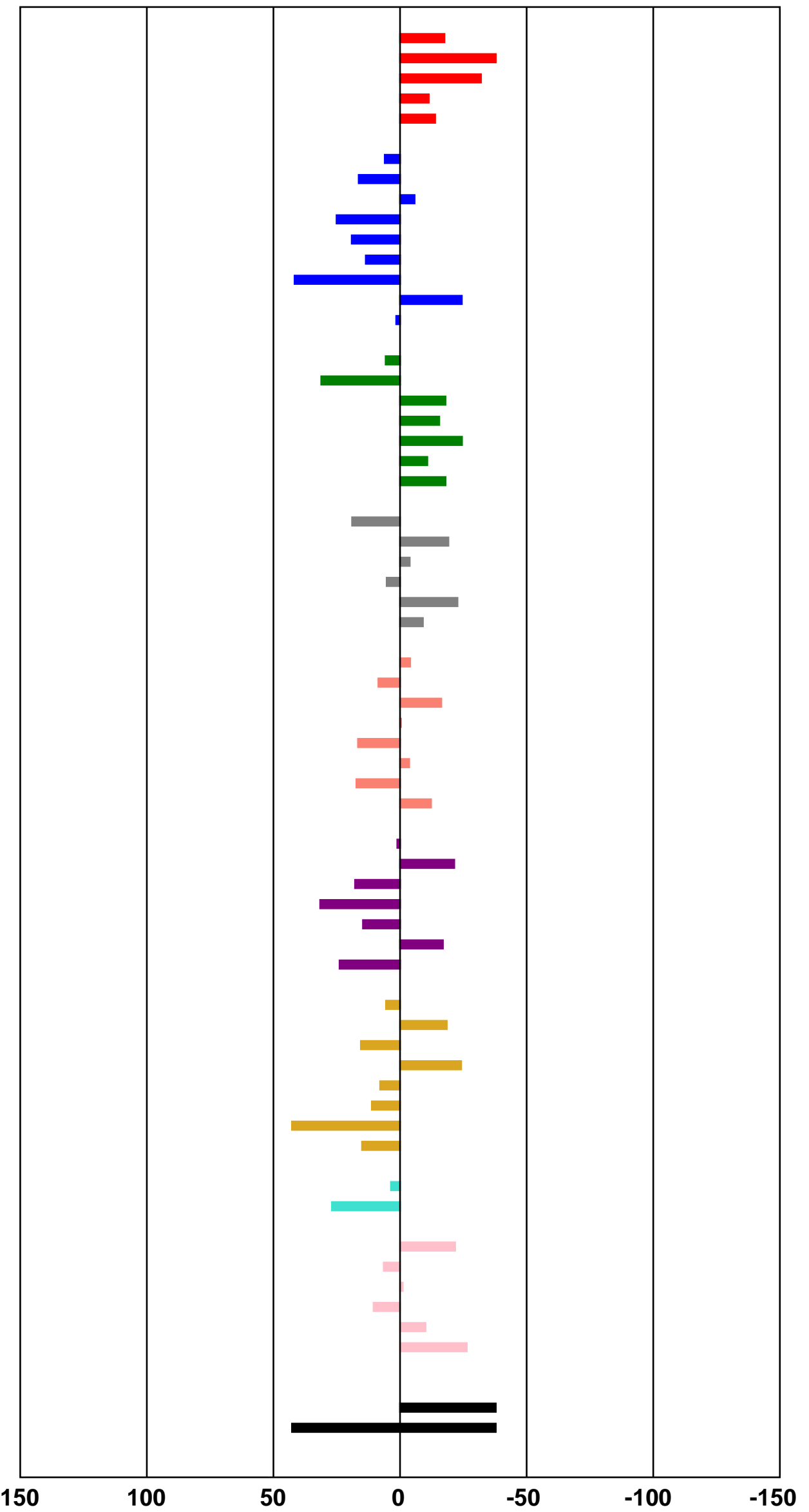


mararas \begin{tabular}{l|l} 
NSC: D-826092 / 1 Conc: 1.00E-5 Molar \\
\hline
\end{tabular} Test Date: Sep 28, 2020 One Dose Mean Graph

\section{Panel/Cell Line}

Leukemia

CCRF-CEM

HL-60(TB)

$\mathrm{K}-562$

MOLT-4

SR

Non-Small Cell Lung Cancer

A549/ATCC

EKVX

HOP-62

HOP-92

$\mathrm{NCl}-\mathrm{H} 226$

$\mathrm{NCl}-\mathrm{H} 23$

$\mathrm{NCl}-\mathrm{H} 322 \mathrm{M}$

$\mathrm{NCl}-\mathrm{H} 460$

$\mathrm{NCl}-\mathrm{H} 522$

Colon Cancer

COLO 205

HCC-2998

HCT-116

HCT-15

HT29

KM12

SW-620

CNS Cancer

SF-268

SF-295

SF-539

SNB-19

SNB-75

U251

Melanoma

LOX IMVI

MALME-3M

M14

SK-MEL-2

SK-MEL-28

SK-MEL-5

UACC-257

UACC-62

Ovarian Cancer

IGROV1

OVCAR-3

OVCAR-4

OVCAR-5

OVCAR-8

$\mathrm{NCl} / \mathrm{ADR}$-RES

SK-OV-3

Renal Cancer

786-0

A498

ACHN

CAKI-1

RXF 393

SN12C

TK-10

UO-31

Prostate Cancer

PC-3

DU-145

Breast Cancer

MCF7

MDA-MB-231/ATCC

HS 578T

BT-549

T-47D

MDA-MB-468

\section{Growth Percent}

17.22

8.29

14.37

32.60

29.46

43.10

43.86

43.14

64.57

43.38

42.91

49.93

18.65

33.94

14.16

56.67

31.34

30.90

12.15

24.19

26.12

66.56

17.45

13.75

42.87

$-1.98$

37.36

44.78

60.35

19.86

56.82

69.94

36.88

75.07

39.10

40.70

17.23

63.55

45.60

46.98

18.85

70.20

50.30

15.70

53.25

11.85

8.40

50.55

76.13

52.96

27.00

22.26

20.86

47.50

36.66

58.42

41.67

2.06

36.87

38.85

78.11
Mean Growth Percent - Growth Percent

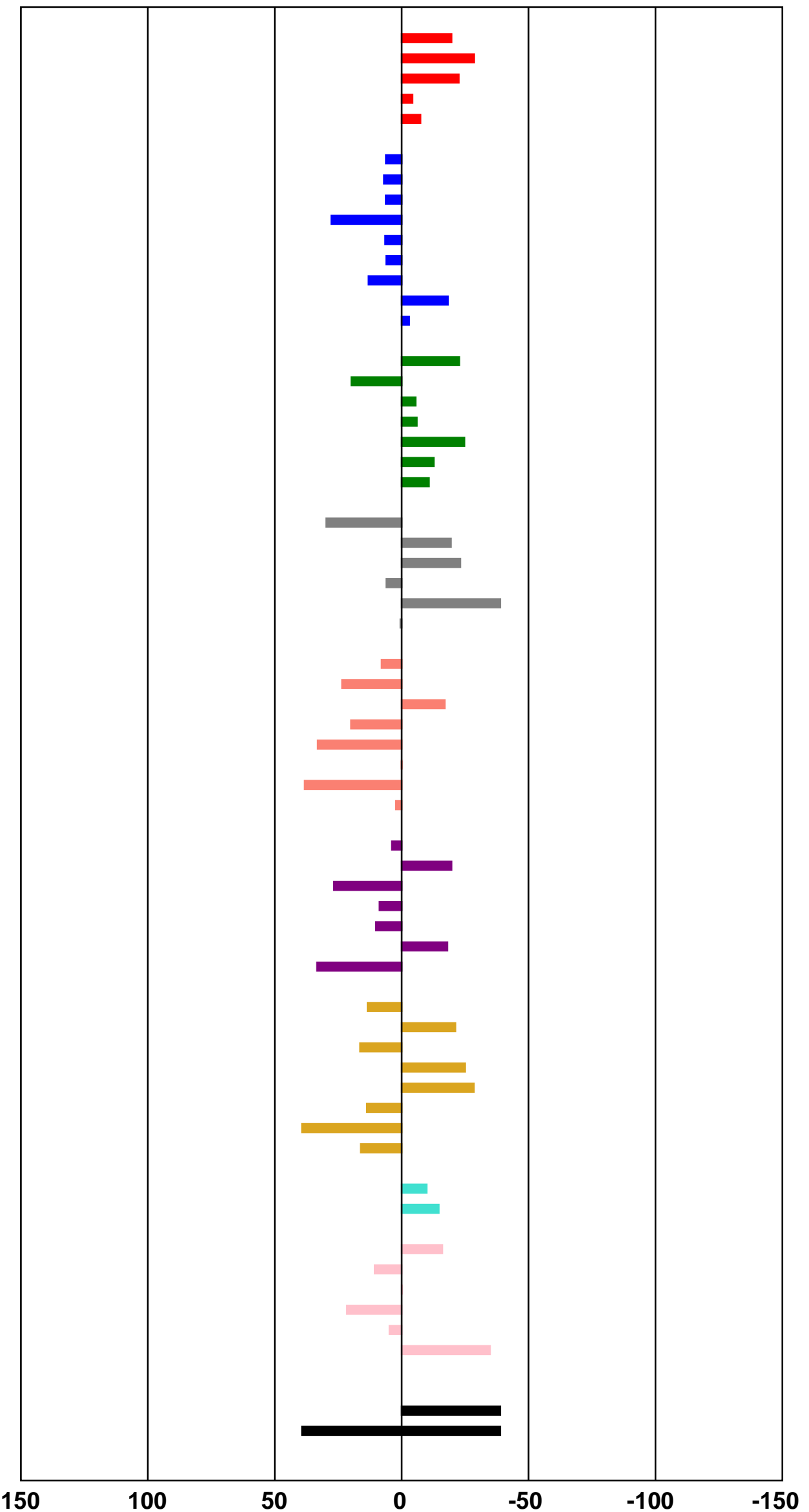




\section{Panel/Cell Line}

Leukemia

CCRF-CEM

HL-60(TB)

K-562

MOLT-4

$\mathrm{SR}$

Non-Small Cell Lung Cancer

A549/ATCC

EKVX

HOP-62

HOP-92

$\mathrm{NCl}-\mathrm{H} 226$

$\mathrm{NCl}-\mathrm{H} 23$

$\mathrm{NCl}-\mathrm{H} 322 \mathrm{M}$

$\mathrm{NCl}-\mathrm{H} 460$

$\mathrm{NCl}-\mathrm{H} 522$

Colon Cancer

COLO 205

HCC-2998

HCT-116

HCT-15

HT29

KM12

SW-620

CNS Cancer

SF-268

SF-295

SF-539

SNB-19

SNB-75

U251

Melanoma

LOX IMVI

MALME-3M

M14

SK-MEL-2

SK-MEL-28

SK-MEL-5

UACC-257

UACC-62

Ovarian Cancer

IGROV1

OVCAR-3

OVCAR-4

OVCAR-5

OVCAR-8

NCI/ADR-RES

SK-OV-3

Renal Cancer

786-0

A498

$\mathrm{ACHN}$

CAKI-1

RXF 393

SN12C

TK-10

UO-31

Prostate Cancer

PC-3

DU-145

Breast Cancer

MCF7

MDA-MB-231/ATCC

HS 578T

BT-549

T-47D

MDA-MB-468

\section{Growth Percent}

20.71

14.10

15.02

38.03

35.98

41.47

47.58

36.19

62.96

49.42

45.27

49.89

16.49

32.84

8.82

67.42

27.32

35.21

13.37

26.10

23.83

73.92

16.78

19.24

44.33

$-4.20$

30.38

43.19

54.96

23.03

51.90

66.70

28.78

70.32

42.62

43.45

15.41

58.34

48.38

44.48

26.77

72.83

43.48

15.45

49.43

12.03

5.28

48.66

72.49

56.81

25.23

24.43

20.20

56.18

30.50

66.84

29.81

13.49

37.07

41.27

78.12
Mean Growth Percent - Growth Percent

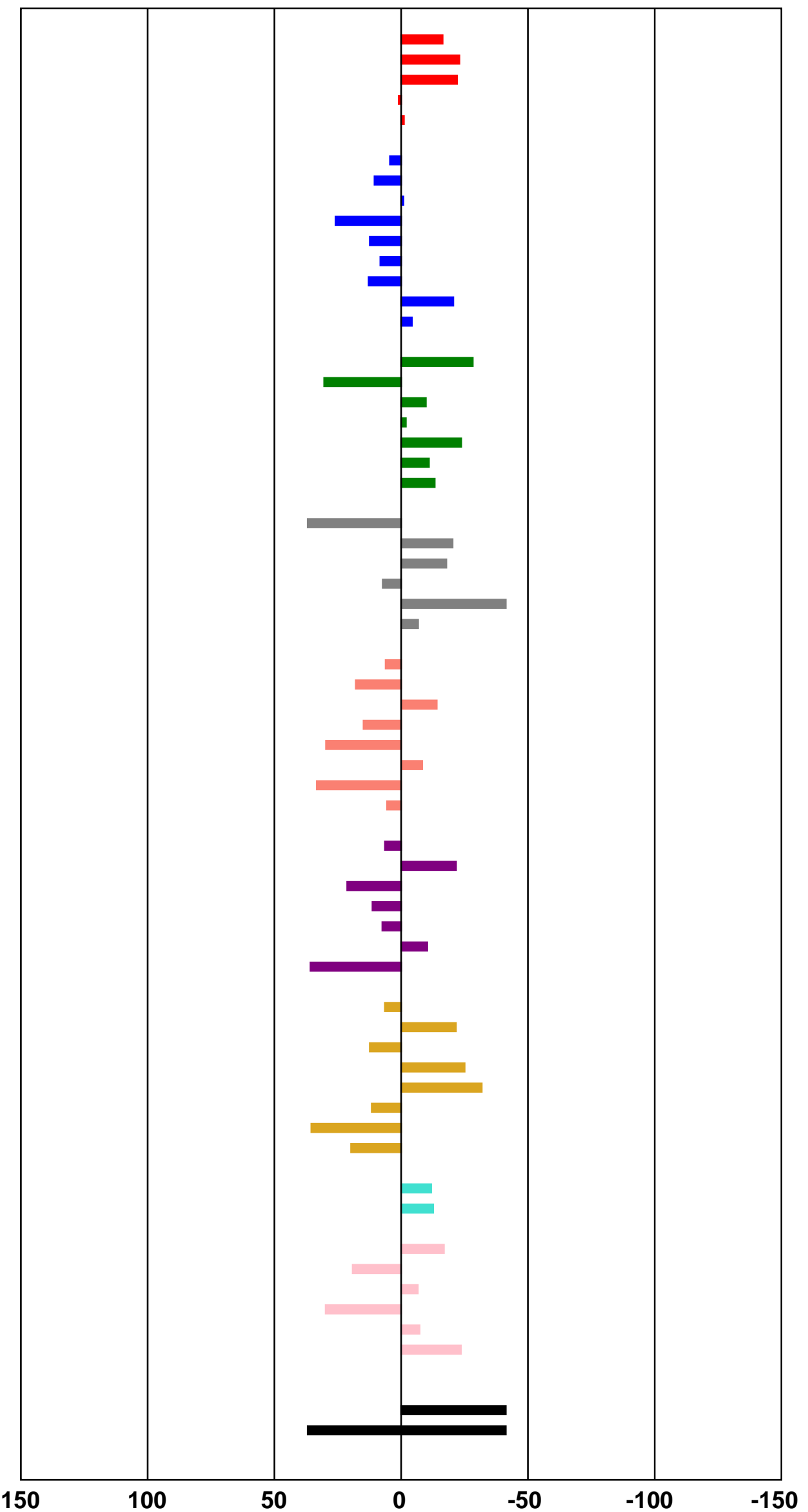


mararas \begin{tabular}{l|l} 
NSC: D-826496/1 Conc: $1.00 E-5$ Molar
\end{tabular} Test Date: Oct 26, 2020 One Dose Mean Graph

\section{Panel/Cell Line}

Leukemia

CCRF-CEM

HL-60(TB)

K-562

MOLT-4

SR

Non-Small Cell Lung Cancer

A549/ATCC

EKVX

HOP-62

HOP-92

$\mathrm{NCl}-\mathrm{H} 226$

$\mathrm{NCl}-\mathrm{H} 23$

$\mathrm{NCl}-\mathrm{H} 322 \mathrm{M}$

$\mathrm{NCl}-\mathrm{H} 460$

$\mathrm{NCl}-\mathrm{H} 522$

Colon Cancer

COLO 205

HCC-2998

HCT-116

HCT-15

HT29

KM12

SW-620

CNS Cancer

SF-268

SF-295

SF-539

SNB-19

U251

Melanoma

MALME-3M

M14

MDA-MB-435

SK-MEL-2

SK-MEL-28

SK-MEL-5

UACC-257

UACC-62

Ovarian Cancer

IGROV1

OVCAR-3

OVCAR-4

OVCAR-5

OVCAR-8

NCI/ADR-RES

SK-OV-3

Renal Cancer

786-0

ACHN

CAKI-1

RXF 393

SN12C

TK-10

UO-31

Prostate Cancer

PC-3

DU-145

Breast Cancer

MCF7

MDA-MB-231/ATCC

HS 578T

BT-549

T-47D

MDA-MB-468

\section{Growth Percent}

19.10

1.38

11.57

40.75

32.95

48.10

42.61

43.78

62.36

57.78

37.69

45.82

22.19

30.59

22.93

63.83

28.28

30.46

14.24

27.37

25.48

65.06

$-2.36$

11.47

40.11

30.07

48.84

24.62

3.85

63.32

61.97

31.52

66.80

30.32

36.69

8.32

59.95

54.62

46.39

14.25

66.18

46.33

45.13

39.30

10.83

43.47

75.64

48.66

22.70

25.96

25.37

40.95

40.02

35.95

32.50

3.98

35.86

38.22

78.00
Mean Growth Percent - Growth Percent

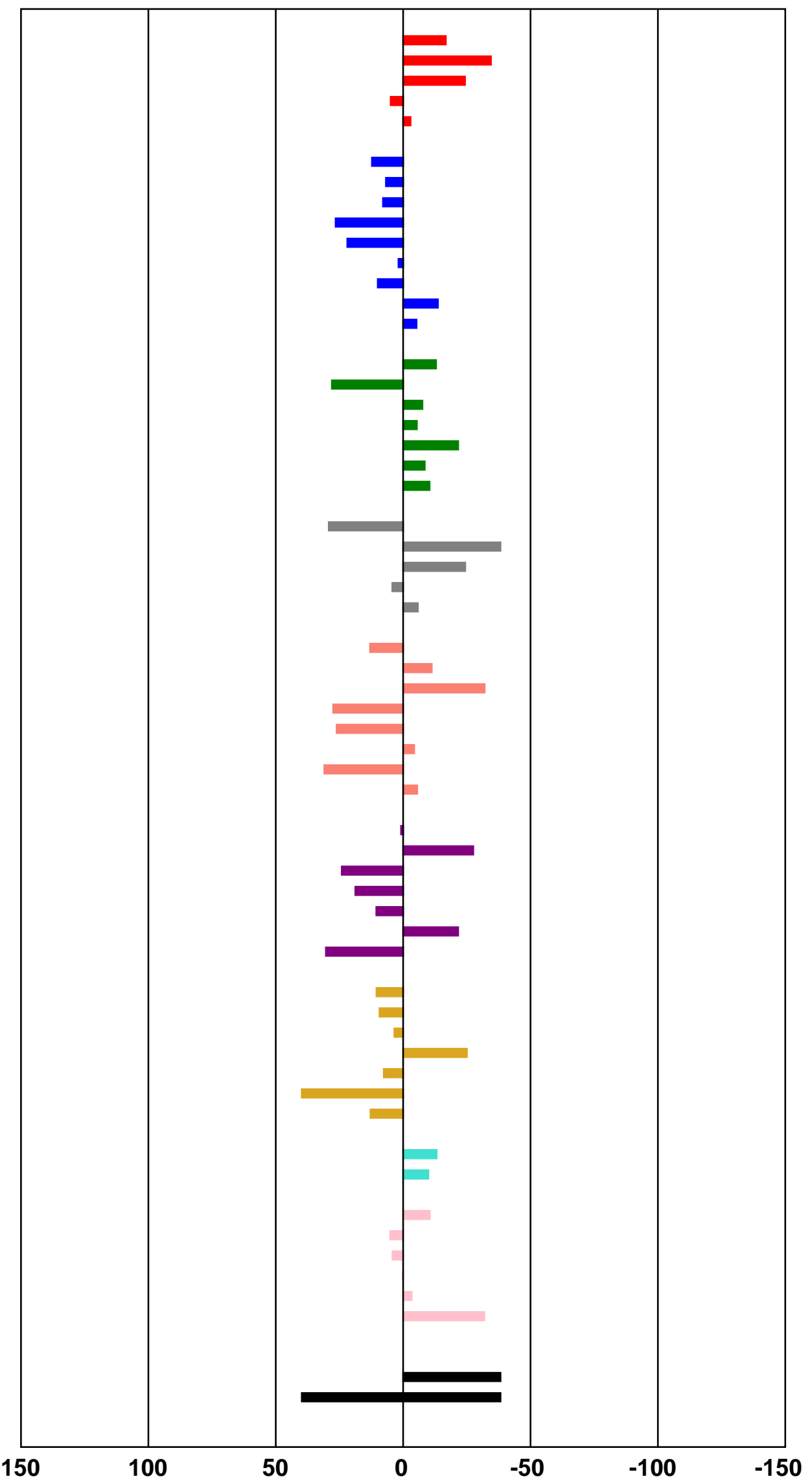


mara a NSC: D-826497/1 Conc: 1.00E-5 Molar Test Date: Oct 26, 2020 One Dose Mean Graph

\section{Panel/Cell Line}

Leukemia

CCRF-CEM

HL-60(TB)

$\mathrm{K}-562$

MOLT-4

SR

Non-Small Cell Lung Cancer

A549/ATCC

EKVX

HOP-62

$\mathrm{NCl}-\mathrm{H} 226$

$\mathrm{NCl}-\mathrm{H} 23$

$\mathrm{NCl}-\mathrm{H} 322 \mathrm{M}$

$\mathrm{NCl}-\mathrm{H} 460$

$\mathrm{NCl}-\mathrm{H} 522$

Colon Cancer

COLO 205

HCC-2998

HCT-116

HCT-15

HT29

KM12

SW-620

CNS Cancer

SF-268

SF-295

SF-539

SNB-19

U251

Melanoma

MALME-3M

M14

MDA-MB-435

SK-MEL-2

SK-MEL-28

SK-MEL-5

UACC-257

UACC-62

Ovarian Cancer

IGROV1

OVCAR-3

OVCAR-4

OVCAR-5

OVCAR-8

$\mathrm{NCl} / \mathrm{ADR}-\mathrm{RES}$

SK-OV-3

Renal Cancer

786-0

ACHN

CAKI-1

RXF 393

SN12C

TK-10

UO-31

Prostate Cancer

PC-3

DU-145

Breast Cancer

MCF7

MDA-MB-231/ATCC

HS 578T

BT-549

T-47D

MDA-MB-468

Mean

Delta

Range

\section{Growth Percent}

26.99

3.52

12.27

31.46

26.64

43.80

43.18

43.30

55.90

42.02

64.53

23.45

34.41

35.27

73.48

19.88

20.34

14.03

23.14

21.17

53.55

9.20

33.01

38.12

27.38

46.19

22.77

0.42

53.48

51.19

30.00

55.03

33.22

40.57

7.46

36.25

60.71

46.81

16.63

62.14

46.24

43.60

40.81

34.93

36.84

73.76

45.53

37.94

44.57

22.28

46.18

45.49

42.09

31.67

$-3.85$

35.84

39.69

77.61
Mean Growth Percent - Growth Percent

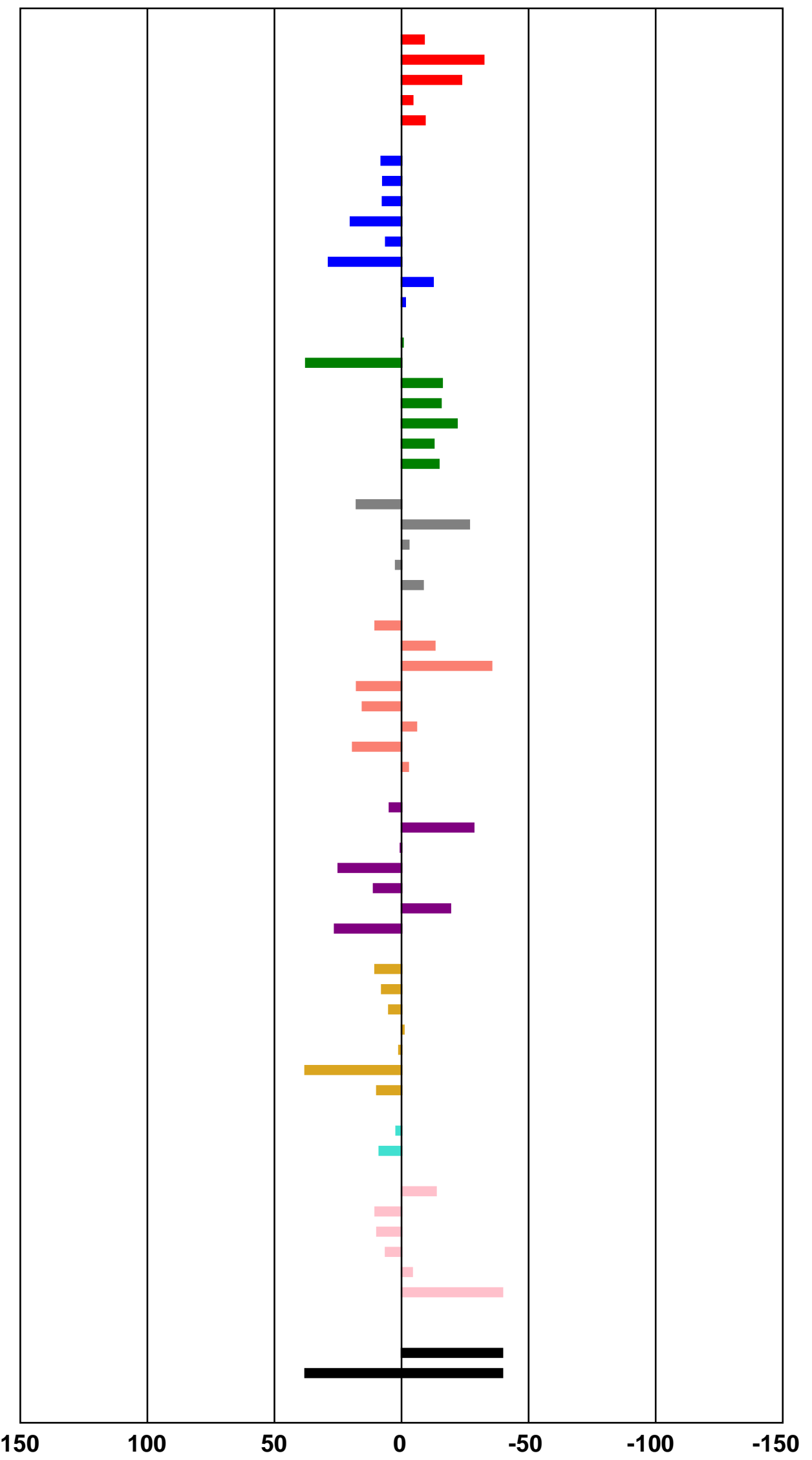


Developmental Therapeutics Program One Dose Mean Graph
NSC: D-826498 / 1

Experiment ID: 20100559
Test Date: Oct 26, 2020

Report Date: Nov 26, 2020

\section{Panel/Cell Line}

Leukemia

CCRF-CEM

HL-60(TB)

$\mathrm{K}-562$

MOLT-4

SR

Non-Small Cell Lung Cancer

A549/ATCC

EKVX

HOP-62

HOP-92

$\mathrm{NCl}-\mathrm{H} 226$

$\mathrm{NCl}-\mathrm{H} 23$

$\mathrm{NCl}-\mathrm{H} 322 \mathrm{M}$

$\mathrm{NCl}-\mathrm{H} 460$

$\mathrm{NCl}-\mathrm{H} 522$

Colon Cancer

COLO 205

HCC-2998

HCT-116

HCT-15

HT29

KM12

SW-620

CNS Cancer

SF-268

SF-295

SF-539

SNB-19

U251

Melanoma

MALME-3M

M14

MDA-MB-435

SK-MEL-2

SK-MEL-28

SK-MEL-5

UACC-257

UACC-62

Ovarian Cancer

IGROV1

OVCAR-3

OVCAR-4

OVCAR-5

OVCAR-8

NCI/ADR-RES

SK-OV-3

Renal Cancer

786-0

ACHN

CAKI-1

RXF 393

SN12C

TK-10

UO-31

Prostate Cancer

PC-3

DU-145

Breast Cancer

MCF7

MDA-MB-231/ATCC

HS 578T

BT-549

T-47D

MDA-MB-468

\section{Growth Percent}

18.63

$-4.23$

8.81
25.89

27.07

31.77

39.43

41.70

66.51

60.49

35.68

46.66

16.16

17.91

2.78

19.77

18.37

21.83

10.74

15.43

28.04

61.72

1.04

6.15

36.03

23.86

65.44

13.08

$-23.78$

78.11

55.44

32.48

68.15

31.48

33.20

$-24.35$

66.23

43.60

38.66

10.09

72.82

49.37

46.12

37.08

$-0.92$

39.56

83.50

43.75

17.36

24.65

24.95

35.35

35.62

47.99

36.14

11.28

31.62

55.97

107.85
Mean Growth Percent - Growth Percent

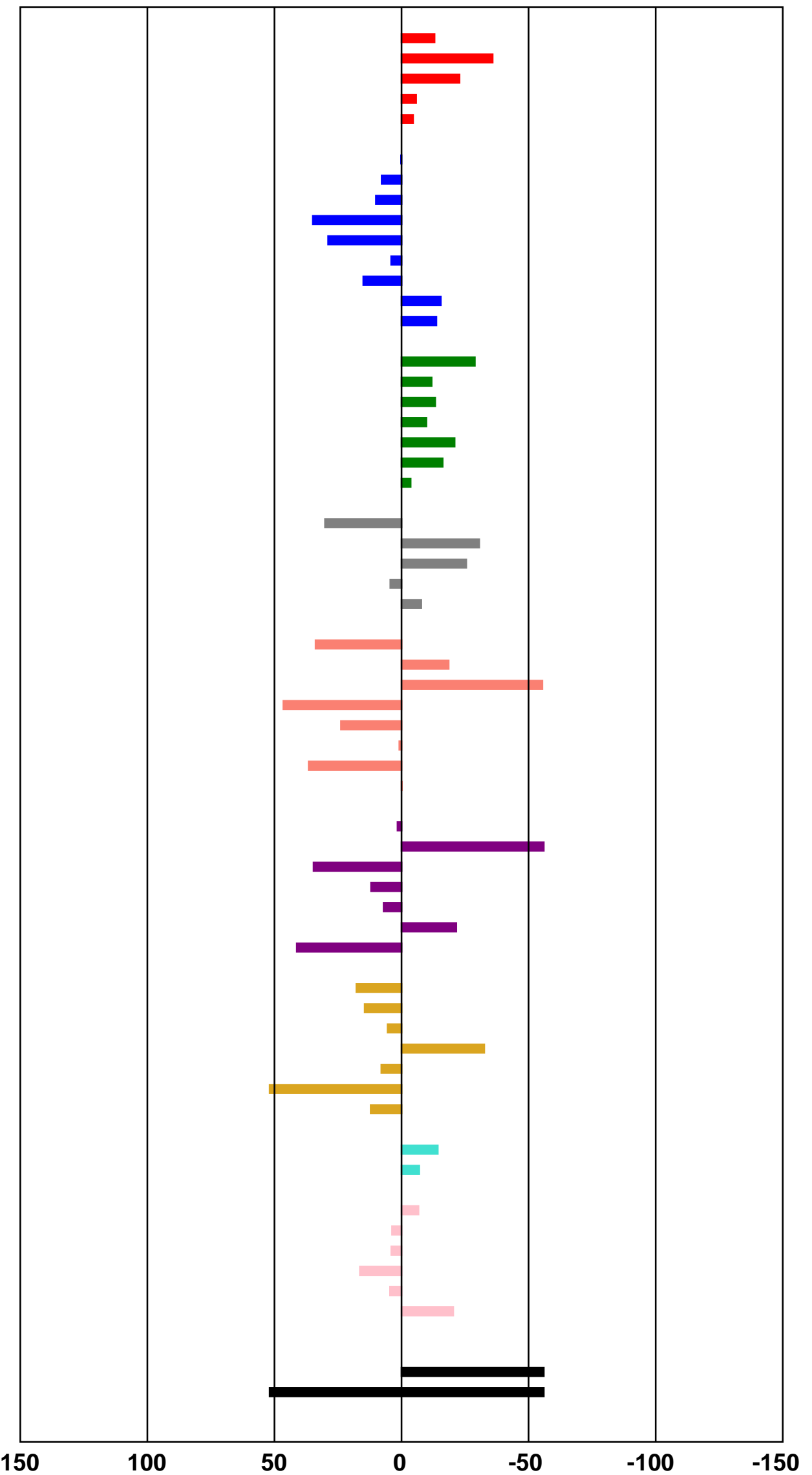


Developmental Therapeutics Program One Dose Mean Graph
NSC: D-826499 / 1

Experiment ID: 20100559
Test Date: Oct 26, 2020

Report Date: Nov 26, 2020

\section{Panel/Cell Line}

Leukemia

CCRF-CEM

HL-60(TB)

$\mathrm{K}-562$

MOLT-4

SR

Non-Small Cell Lung Cancer

A549/ATCC

EKVX

HOP-62

HOP-92

$\mathrm{NCl}-\mathrm{H} 226$

$\mathrm{NCl}-\mathrm{H} 23$

$\mathrm{NCl}-\mathrm{H} 322 \mathrm{M}$

$\mathrm{NCl}-\mathrm{H} 460$

$\mathrm{NCl}-\mathrm{H} 522$

Colon Cancer

COLO 205

HCC-2998

HCT-116

HCT-15

HT29

KM12

SW-620

CNS Cancer

SF-268

SF-295

SF-539

SNB-19

U251

Melanoma

MALME-3M

M14

MDA-MB-435

SK-MEL-2

SK-MEL-28

SK-MEL-5

UACC-257

UACC-62

Ovarian Cancer

IGROV1

OVCAR-3

OVCAR-4

OVCAR-5

OVCAR-8

NCI/ADR-RES

SK-OV-3

Renal Cancer

786-0

ACHN

CAKI-1

RXF 393

SN12C

TK-10

UO-31

Prostate Cancer

PC-3

DU-145

Breast Cancer

MCF7

MDA-MB-231/ATCC

HS 578T

BT-549

T-47D

MDA-MB-468

\section{Growth Percent}

20.78

1.67

8.06
26.07

27.45

27.76

34.25

34.19

35.57

53.85

19.79

46.90

13.84

8.89

1.45

24.85

19.47

22.40

8.59

18.33

24.73

63.62

$-5.64$

$-1.60$

35.19

22.64

59.21

19.50

$-20.89$

66.95

60.36

21.49

59.07

25.59

32.43

$-10.08$

49.62

43.04

35.78

6.60

58.25

47.00

44.42

39.89

$-3.39$

44.77

53.46

43.20

17.44

23.72

21.39

30.52

34.10

48.81

26.73

$-1.16$

28.05

48.94

87.84
Mean Growth Percent - Growth Percent

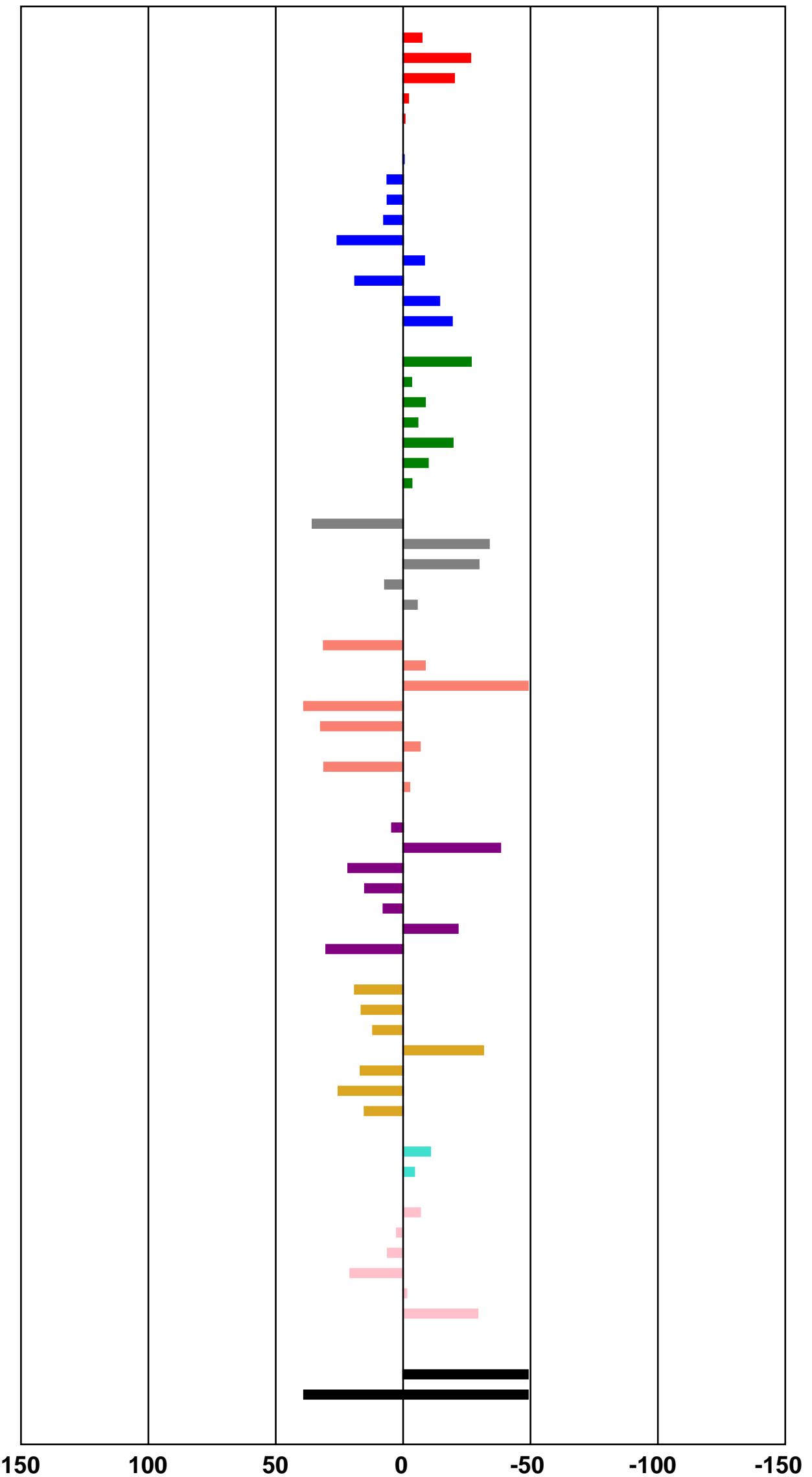




\section{Panel/Cell Line}

Leukemia

CCRF-CEM

HL-60(TB)

K-562

MOLT-4

$\mathrm{SR}$

Non-Small Cell Lung Cancer

A549/ATCC

EKVX

HOP-62

$\mathrm{NCl}-\mathrm{H} 226$

$\mathrm{NCl}-\mathrm{H} 23$

$\mathrm{NCl}-\mathrm{H} 322 \mathrm{M}$

$\mathrm{NCl}-\mathrm{H} 460$

$\mathrm{NCl}-\mathrm{H} 522$

Colon Cancer

COLO 205

HCC-2998

HCT-116

HCT-15

HT29

KM12

SW-620

CNS Cancer

SF-268

SF-295

SF-539

SNB-19

U251

Melanoma

MALME-3M

M14

MDA-MB-435

SK-MEL-2

SK-MEL-28

SK-MEL-5

UACC- 257

UACC-62

Ovarian Cancer

IGROV1

OVCAR-3

OVCAR-4

OVCAR-5

OVCAR-8

NCI/ADR-RES

SK-OV-3

Renal Cancer

786-0

ACHN

CAKI-1

RXF 393

$\mathrm{SN} 12 \mathrm{C}$

TK-10

UO-31

Prostate Cancer

PC-3

DU-145

Breast Cancer

MCF7

MDA-MB-231/ATCC

HS 578T

BT-549

T-47D

MDA-MB-468

\section{Growth Percent}

25.15

9.69

19.76

44.98

34.50

54.56

54.22

52.72

62.45

45.39

64.77

28.83

33.98

50.19

78.56

22.05

30.67

15.95

25.97

17.88

58.90

2.33

21.43

45.92

35.17

47.33

29.60

$-4.66$

71.63

53.82

40.23

58.51

38.95

43.18

$-10.41$

67.04

75.38

62.16

28.12

57.84

40.43

49.19

39.14

$-4.05$

42.51

89.26

50.91

34.01

50.23

23.12

57.54

17.33

34.91

31.74

20.14

39.48

49.89

99.67
Mean Growth Percent - Growth Percent

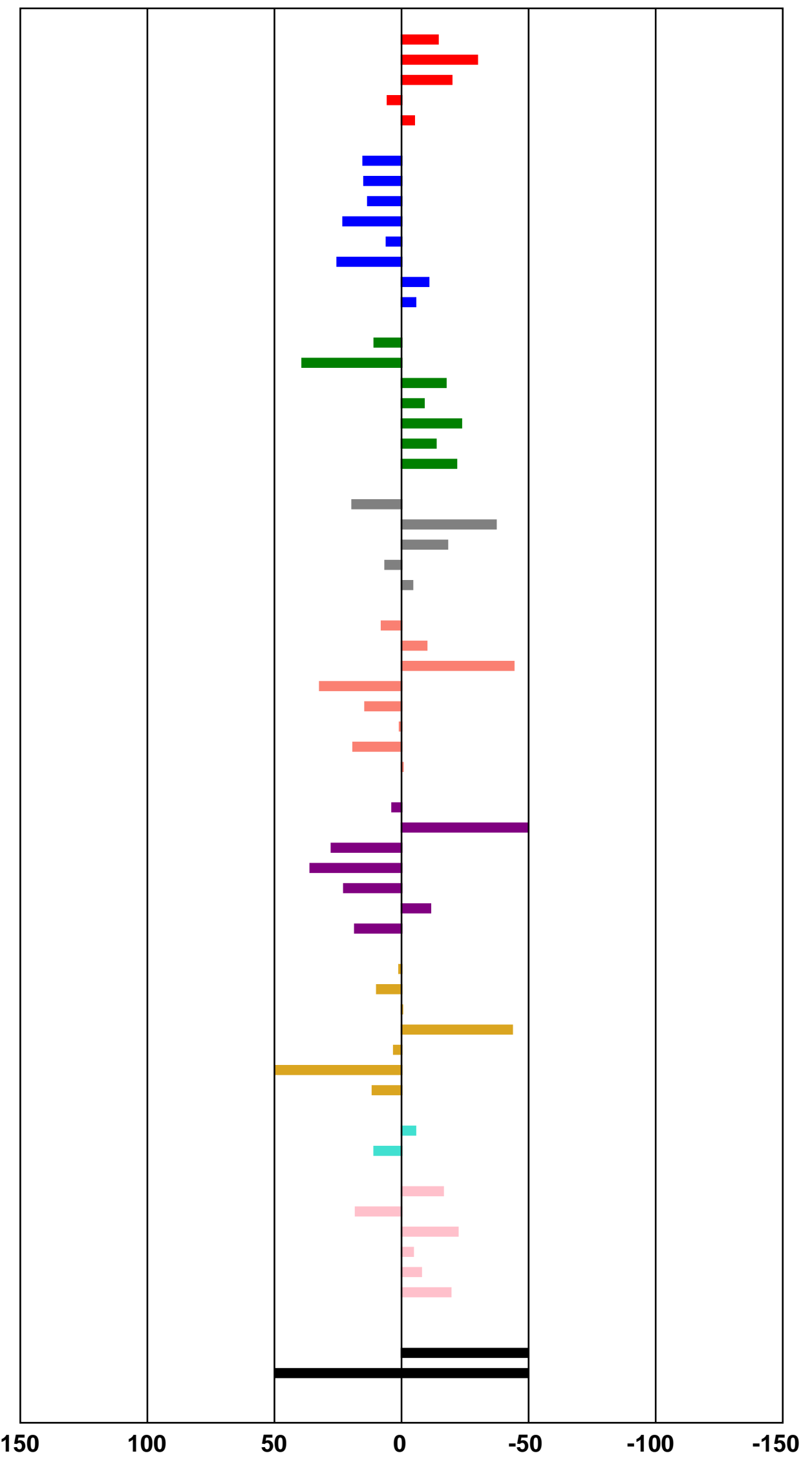


nas a r a \begin{tabular}{l|l} 
NSC: D-826502 / 1 Conc: 1.00E-5 Molar \\
\hline
\end{tabular} Test Date: Oct 26, 2020 One Dose Mean Graph

\section{Panel/Cell Line}

Leukemia

CCRF-CEM

$\mathrm{K}-562$

MOLT-4

RPMI-8226

SR

Non-Small Cell Lung Cancer

A549/ATCC

EKVX

HOP-62

$\mathrm{NCl}-\mathrm{H} 226$

$\mathrm{NCl}-\mathrm{H} 23$

$\mathrm{NCl}-\mathrm{H} 322 \mathrm{M}$

$\mathrm{NCl}-\mathrm{H} 460$

$\mathrm{NCl}-\mathrm{H} 522$

Colon Cancer

COLO 205

HCC-2998

HCT-116

HCT-15

HT29

KM12

SW-620

CNS Cancer

SF-268

SF-295

SF-539

SNB-19

U251

Melanoma

MALME-3M

M14

MDA-MB-435

SK-MEL-2

SK-MEL-28

SK-MEL-5

UACC-257

UACC-62

Ovarian Cancer

IGROV1

OVCAR-3

OVCAR-4

OVCAR-5

OVCAR-8

NCI/ADR-RES

SK-OV-3

Renal Cancer

786-0

ACHN

CAKI-1

RXF 393

SN12C

TK-10

UO-31

Prostate Cancer

PC-3

DU-145

Breast Cancer

MCF7

MDA-MB-231/ATCC

HS 578T

BT-549

T-47D

MDA-MB-468

\section{Growth Percent}

17.61

$-16.16$

8.55

21.78

14.43

21.22

27.15

43.05

39.35

64.59

23.23

40.39

11.19

21.93

9.76

21.25

12.42

14.33

16.77

10.69

24.62

55.50

0.67

7.47

38.79

23.75

61.42

14.53

$-39.88$

72.25

57.70

7.66

69.05

37.21

23.42

7.12

64.06

42.58

28.53

7.22

71.31

50.16

36.94

29.94

7.94

34.30

92.61

43.52

16.45

15.32

21.96

27.61

39.08

21.25

62.42

0.88

28.55

68.43

132.49
Mean Growth Percent - Growth Percent

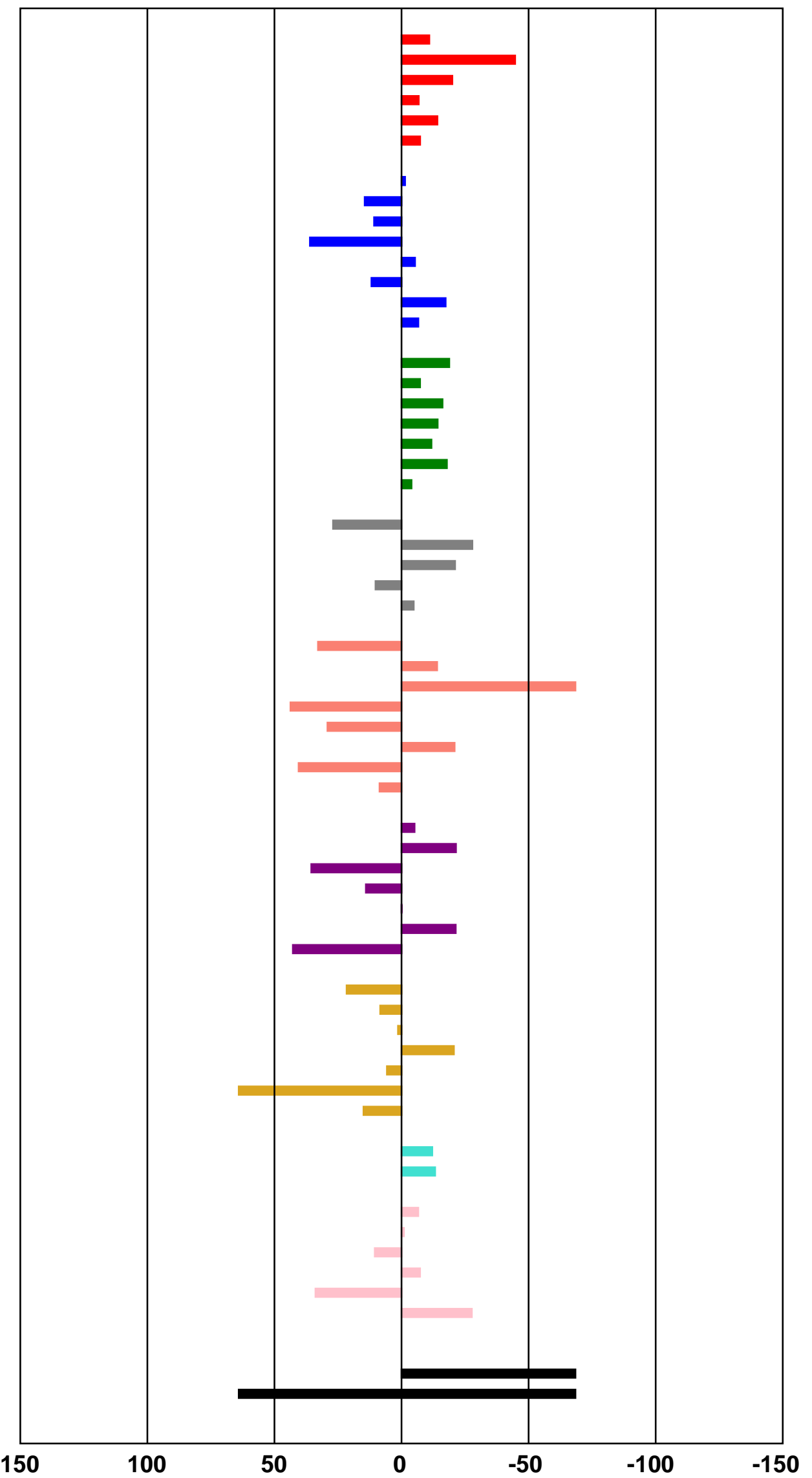




\section{Panel/Cell Line}

Leukemia

CCRF-CEM

HL-60(TB)

$\mathrm{K}-562$

MOLT-4

RPMI-8226

$\mathrm{SR}$

Non-Small Cell Lung Cancer

A549/ATCC

EKVX

HOP-62

HOP-92

$\mathrm{NCl}-\mathrm{H} 226$

$\mathrm{NCl}-\mathrm{H} 23$

$\mathrm{NCl}-\mathrm{H} 322 \mathrm{M}$

$\mathrm{NCl}-\mathrm{H} 460$

$\mathrm{NCl}-\mathrm{H} 522$

Colon Cancer

COLO 205

HCC-2998

HCT-116

HCT-15

HT29

KM12

SW-620

CNS Cancer

SF-268

SF-539

SNB-19

U251

Melanoma

MALME-3M

M14

MDA-MB-435

SK-MEL-2

SK-MEL-28

SK-MEL-5

UACC-257

UACC-62

Ovarian Cancer

IGROV1

OVCAR-3

OVCAR-4

OVCAR-5

OVCAR-8

NCI/ADR-RES

SK-OV-3

Renal Cancer

786-0

$\mathrm{ACHN}$

CAKI-1

RXF 393

SN12C

TK-10

UO-31

Prostate Cancer

PC-3

DU-145

Breast Cancer

MCF7

MDA-MB-231/ATCC

HS 578T

BT-549

T-47D

MDA-MB-468

\section{Growth Percent}

16.36

$-15.77$

10.32

25.64

14.07

20.10

31.06

46.94

44.06

81.14

60.55

30.52

45.57

17.18

25.60

6.42

22.30

18.67

17.89

13.93

18.54

32.88

64.17

$-12.95$

4.96

36.87

23.26

65.30

14.58

$-26.22$

77.63

56.73

26.84

70.86

30.54

27.49

6.57

70.40

49.46

38.78

9.11

72.14

50.27

43.02

38.99

$-8.88$

37.27

91.92

44.48

17.76

23.49

23.74

28.42

37.35

46.16

54.10

$-8.39$

31.76

57.98

118.14
Mean Growth Percent - Growth Percent

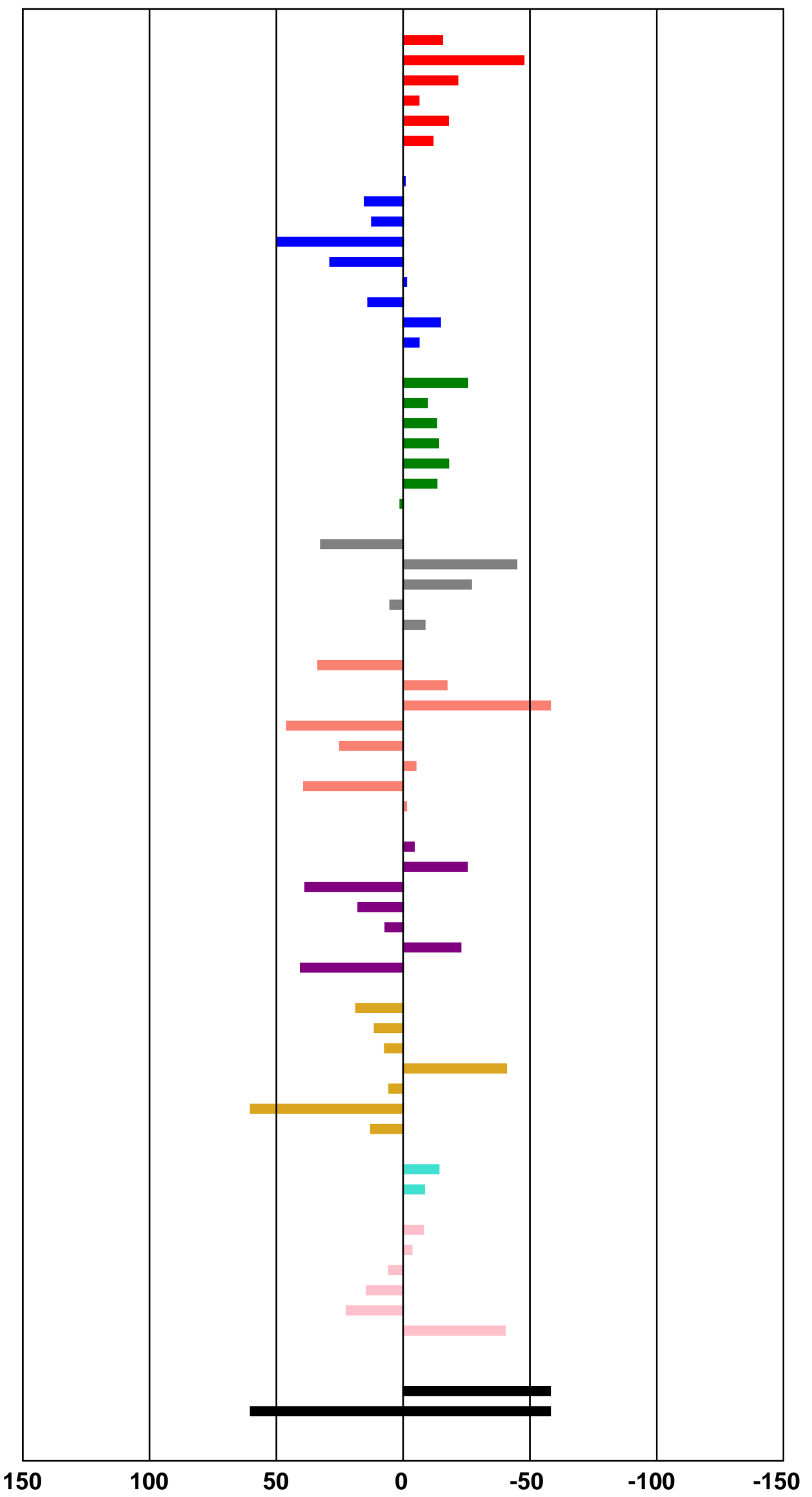




\section{Panel/Cell Line}

Leukemia

CCRF-CEM

HL-60(TB)

$\mathrm{K}-562$

MOLT-4

SR

Non-Small Cell Lung Cancer

A549/ATCC

EKVX

HOP-62

HOP-92

$\mathrm{NCl}-\mathrm{H} 226$

$\mathrm{NCl}-\mathrm{H} 23$

$\mathrm{NCl}-\mathrm{H} 322 \mathrm{M}$

$\mathrm{NCl}-\mathrm{H} 460$

$\mathrm{NCl}-\mathrm{H} 522$

Colon Cancer

COLO 205

HCC-2998

HCT-116

HCT-15

HT29

KM12

SW-620

CNS Cancer

SF-268

SF-295

SF-539

SNB-19

SNB-75

U251

Melanoma

LOX IMVI

MALME-3M

M14

SK-MEL-2

SK-MEL-28

SK-MEL-5

UACC-257

UACC-62

Ovarian Cancer

IGROV1

OVCAR-3

OVCAR-4

OVCAR-5

OVCAR-8

$\mathrm{NCl} / \mathrm{ADR}$-RES

SK-OV-3

Renal Cancer

786-0

A498

ACHN

CAKI-1

RXF 393

SN12C

TK-10

UO-31

Prostate Cancer

PC-3

DU-145

Breast Cancer

MCF7

MDA-MB-231/ATCC

HS 578T

BT-549

T-47D

MDA-MB-468

\section{Growth Percent}

17.11

$-15.93$

10.39

16.76

8.67

24.31

47.03

45.55

63.92

14.50

23.76

55.04

8.85

30.77

$-7.11$

22.73

10.56

15.61

15.00

8.94

25.80

40.19

18.03

10.36

44.36

43.02

32.26

12.91

77.80

24.88

63.72

65.25

10.22

66.09

37.29

28.85

9.18

54.68

38.25

30.73

13.58

84.13

31.93

12.79

33.61

5.60

46.46

29.77

75.84

47.32

22.32

15.88

14.99

22.31

49.31

35.06

58.88

22.69

30.57

46.50

100.06
Mean Growth Percent - Growth Percent

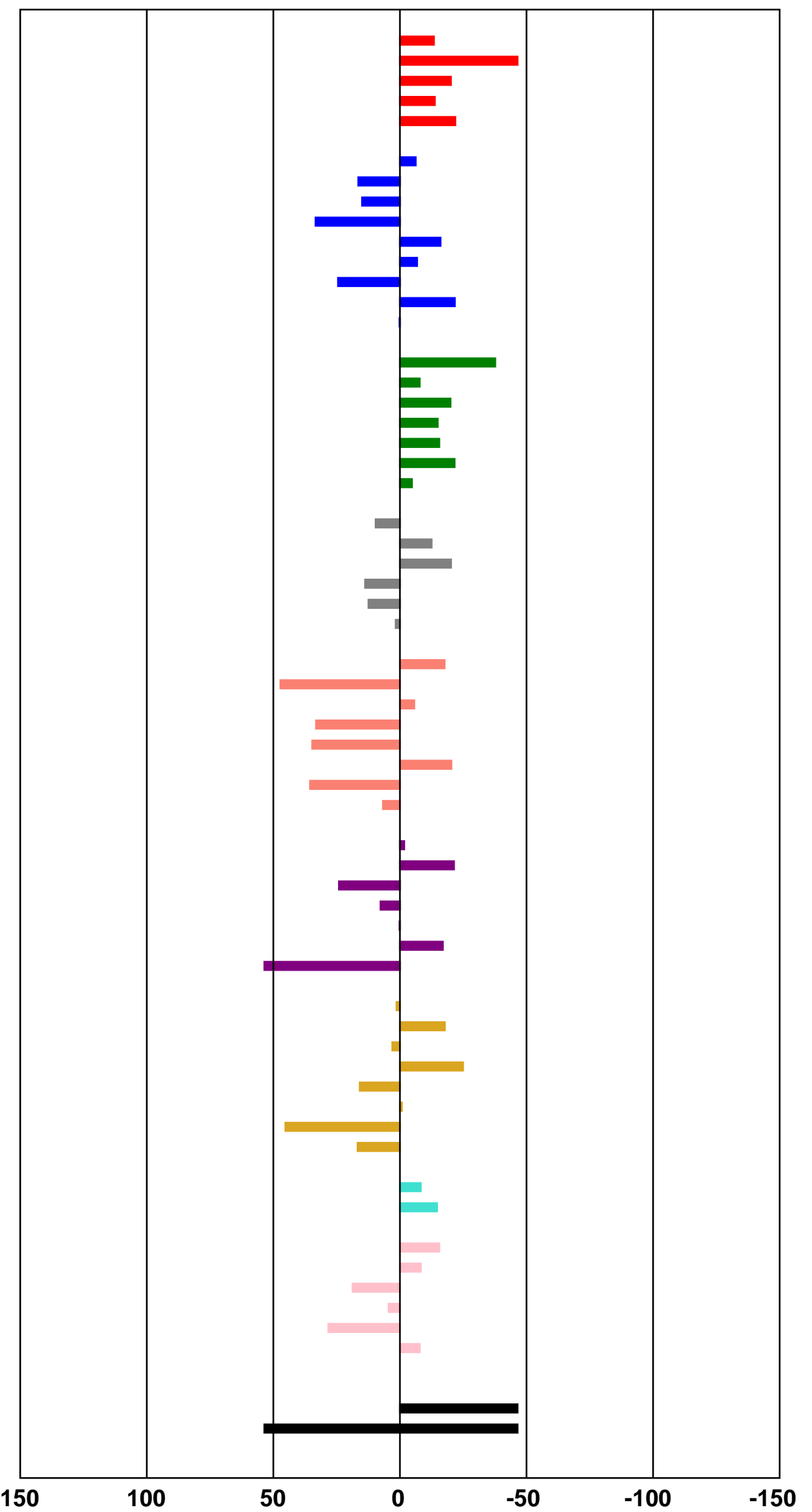




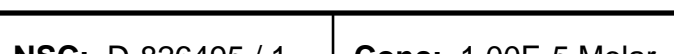
NSC: D-826495/1 Conc: $1.00 E-5$ Molar Test Date: Oct 26, 2020

Experiment ID: 20100559

\section{Panel/Cell Line}

Leukemia

CCRF-CEM

$\mathrm{K}-562$

MOLT-4

RPMI-8226

SR

Non-Small Cell Lung Cancer

A549/ATCC

EKVX

HOP-62

HOP-92

$\mathrm{NCl}-\mathrm{H} 226$

$\mathrm{NCl}-\mathrm{H} 23$

$\mathrm{NCl}-\mathrm{H} 322 \mathrm{M}$

$\mathrm{NCl}-\mathrm{H} 460$

$\mathrm{NCl}-\mathrm{H} 522$

Colon Cancer

COLO 205

HCC-2998

HCT-116

HCT-15

HT29

KM12

SW-620

CNS Cancer

SF-268

SF-539

SNB-19

U251

Melanoma

MALME-3M

M14

MDA-MB-435

SK-MEL-2

SK-MEL-28

SK-MEL-5

UACC-257

UACC-62

Ovarian Cancer

IGROV1

OVCAR-3

OVCAR-4

OVCAR-5

OVCAR-8

$\mathrm{NCl} / \mathrm{ADR}$-RES

SK-OV-3

Renal Cancer

786-0

$\mathrm{ACHN}$

CAKI-1

RXF 393

SN12C

TK-10

UO-31

Prostate Cancer

PC-3

DU-145

Breast Cancer

MCF7

MDA-MB-231/ATCC

HS 578T

BT-549

T-47D

MDA-MB-468

\section{Growth Percent}

18.02

$-25.10$

7.96

23.46

15.86

34.37

26.31

43.05

41.27

87.15

62.42

20.02

46.54

16.79

16.84

4.79

16.41

14.45

17.68

11.03

23.16

27.89

65.94

$-0.10$

1.56

37.34

22.68

63.77

8.20

$-12.13$

69.54

58.13

29.99

67.26

28.47

29.89

23.67

70.14

46.68

33.22

2.50

73.67

52.88

38.91

36.93

6.23

30.19

87.62

44.38

22.44

26.18

21.12

29.95

35.77

19.27

55.11

11.70

31.39

56.49

112.72
Mean Growth Percent - Growth Percent

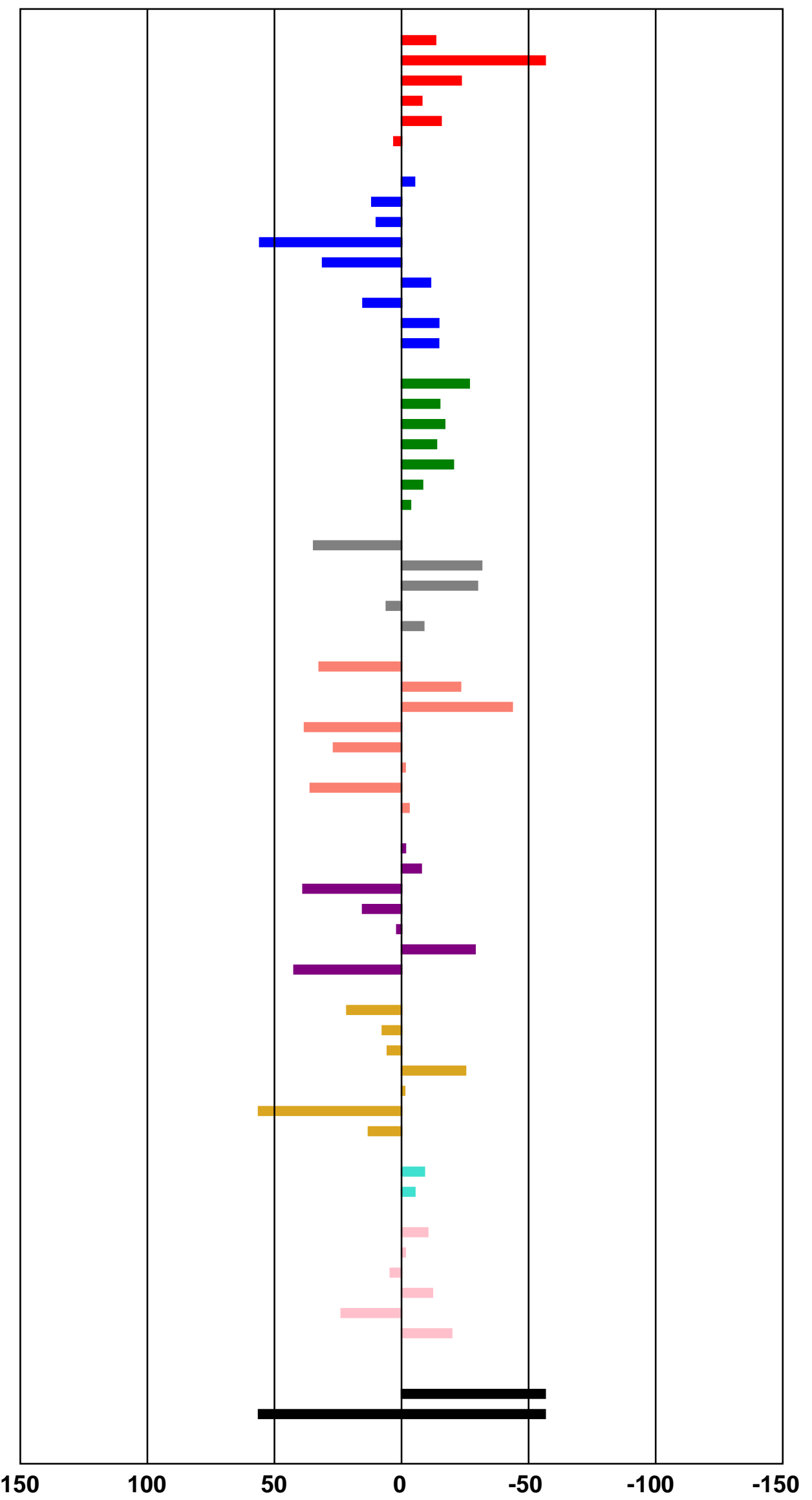


In-Vitro Testing Results

\begin{tabular}{|l|l|l|l|}
\hline NSC : D - 810513/1 & Experiment ID : 1901NS52 & Test Type : 08 & Units : Molar \\
\hline Report Date : February 02, 2019 & Test Date : January 14, 2019 & QNS : & MC : \\
\hline COMI : 2351756843 & Stain Reagent : SRB Dual-Pass Related & SSPL : OM6Y & \\
\hline
\end{tabular}

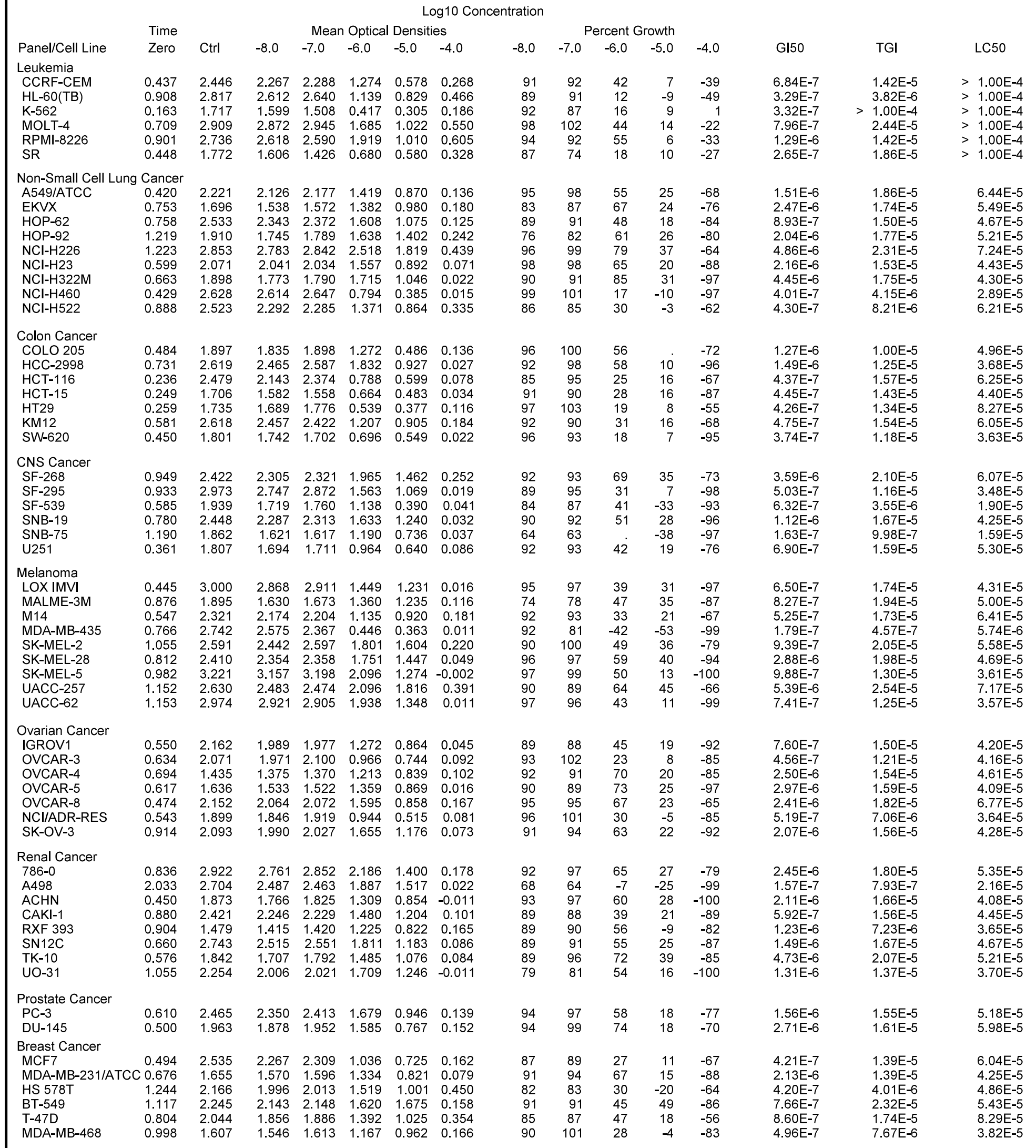


In-Vitro Testing Results

\begin{tabular}{|l|l|l|l|}
\hline NSC : D - 810520 /1 & Experiment ID : 1901NS56 & Test Type : 08 & Units : Molar \\
\hline Report Date : February 24, 2019 & Test Date : January 28, 2019 & QNS : & MC : \\
\hline COMI : 2351710827 & Stain Reagent : SRB Dual-Pass Related & SSPL : OM6Y & \\
\hline
\end{tabular}

\begin{tabular}{|c|c|c|c|c|c|c|c|c|c|c|c|c|c|c|c|c|}
\hline \multirow[b]{3}{*}{ Panel/Cell Line } & \multicolumn{13}{|c|}{ Log10 Concentration } & & \\
\hline & \multirow{2}{*}{$\begin{array}{l}\text { Time } \\
\text { Zero }\end{array}$} & & & Mean & Optical & Densitie & & & & reent & rowth & & & & & \\
\hline & & Ctrl & -8.0 & -7.0 & -6.0 & -5.0 & -4.0 & -8.0 & -7.0 & -6.0 & -5.0 & -4.0 & GI50 & TGI & & LC50 \\
\hline Leukemia & & & & & & & & & & & & & & & & \\
\hline CCRF-CEM & 0.580 & 3.025 & 3.006 & 2.945 & 1.269 & 0.634 & 0.657 & 99 & 97 & 28 & 2 & 3 & $4.80 \mathrm{E}-7$ & $>1.00 \mathrm{E}-4$ & $>$ & $1.00 \mathrm{E}-4$ \\
\hline HL-60(TB) & 0.877 & 3.263 & 3.239 & 3.207 & 0.847 & 0.718 & 0.663 & 99 & 98 & -3 & -18 & -24 & $2.96 \mathrm{E}-7$ & 9.25E-7 & $>$ & $1.00 \mathrm{E}-4$ \\
\hline $\mathrm{K}-562$ & 0.214 & 2.584 & 2.555 & 2.321 & 0.590 & 0.346 & 0.392 & 99 & 89 & 16 & 6 & 8 & $3.41 \mathrm{E}-7$ & $>1.00 \mathrm{E}-4$ & $>$ & $1.00 \mathrm{E}-4$ \\
\hline MOLT-4 & 0.550 & 2.896 & 2.937 & 2.817 & 1.462 & 0.676 & 0.490 & 102 & 97 & 39 & 5 & -11 & $6.42 \mathrm{E}-7$ & $2.13 \mathrm{E}-5$ & $>$ & $1.00 \mathrm{E}-4$ \\
\hline RPMI-8226 & 1.128 & 3.118 & 3.126 & 3.102 & 2.143 & 0.898 & 1.140 & 100 & 99 & 51 & -20 & 1 & $1.03 \mathrm{E}-6$ & & $>$ & $1.00 \mathrm{E}-4$ \\
\hline SR & 0.281 & 1.647 & 1.437 & 1.185 & 0.559 & 0.394 & 0.408 & 85 & 66 & 20 & 8 & 9 & $2.25 \mathrm{E}-7$ & $>1.00 \mathrm{E}-4$ & $>$ & $1.00 \mathrm{E}-4$ \\
\hline Non-Small Cell Lung & Cancer & & & & & & & & & & & & & & & \\
\hline A549/ATCC & 0.373 & 2.457 & 2.255 & 2.241 & 1.283 & 0.463 & 0.401 & 90 & 90 & 44 & 4 & 1 & $7.28 \mathrm{E}-7$ & $>1.00 \mathrm{E}-4$ & $>$ & $1.00 \mathrm{E}-4$ \\
\hline EKVX & 0.679 & 2.007 & 1.886 & 1.820 & 1.416 & 0.617 & 0.266 & 91 & 86 & 55 & -9 & -61 & $1.21 \mathrm{E}-6$ & $7.21 \mathrm{E}-6$ & & $6.15 \mathrm{E}-5$ \\
\hline HOP-62 & 0.451 & 2.034 & 1.934 & 1.858 & 1.109 & 0.286 & 0.420 & 94 & 89 & 42 & -37 & -7 & $6.63 \mathrm{E}-7$ & $3.40 \mathrm{E}-6$ & $>$ & $1.00 \mathrm{E}-4$ \\
\hline HOP-92 & 1.205 & 1.972 & 1.877 & 1.783 & 1.586 & 0.713 & 0.539 & 88 & 75 & 50 & -41 & -55 & $9.73 \mathrm{E}-7$ & $3.54 \mathrm{E}-6$ & & 4.30E-5 \\
\hline $\mathrm{NCl}-\mathrm{H} 226$ & 1.465 & 3.101 & 3.068 & 2.965 & 2.534 & 0.735 & 0.924 & 98 & 92 & 65 & -50 & -37 & $1.36 \mathrm{E}-6$ & 3.69E-6 & $>$ & $1.00 \mathrm{E}-4$ \\
\hline $\mathrm{NCl}-\mathrm{H} 23$ & 0.538 & 1.664 & 1.610 & 1.563 & 1.197 & 0.426 & 0.261 & 95 & 91 & 59 & -21 & -52 & $1.28 \mathrm{E}-6$ & $5.46 \mathrm{E}-6$ & & $8.88 \mathrm{E}-5$ \\
\hline $\mathrm{NCl}-\mathrm{H} 322 \mathrm{M}$ & 0.694 & 2.018 & 1.961 & 1.950 & 1.478 & 0.614 & 0.121 & 96 & 95 & 59 & -12 & -83 & $1.35 \mathrm{E}-6$ & $6.86 \mathrm{E}-6$ & & 3.47E-5 \\
\hline $\mathrm{NCl}-\mathrm{H} 460$ & 0.284 & 2.974 & 3.050 & 3.017 & 0.605 & 0.099 & 0.214 & 103 & 102 & 12 & -65 & -25 & $3.76 \mathrm{E}-7$ & $1.43 \mathrm{E}-6$ & & \\
\hline $\mathrm{NCl}-\mathrm{H} 522$ & 0.911 & 2.836 & 2.640 & 2.504 & 1.421 & 0.743 & 0.751 & 90 & 83 & 26 & -18 & -18 & 3.82E-7 & $3.88 \mathrm{E}-6$ & $>$ & $1.00 \mathrm{E}-4$ \\
\hline Colon Cancer & & & & & & & & & & & & & & & & \\
\hline COLO 205 & 0.443 & 2.075 & 1.975 & 2.045 & 1.099 & 0.159 & 0.415 & 94 & 98 & 40 & -64 & -6 & $6.78 \mathrm{E}-7$ & $2.43 \mathrm{E}-6$ & & \\
\hline HCC-2998 & 0.652 & 2.525 & 2.492 & 2.406 & 1.471 & 0.144 & 0.203 & 98 & 94 & 44 & -78 & -69 & $7.48 \mathrm{E}-7$ & $2.29 \mathrm{E}-6$ & & $5.89 \mathrm{E}-6$ \\
\hline НСТ-116 & 0.242 & 2.422 & 2.395 & 2.332 & 0.653 & 0.124 & 0.233 & 99 & 96 & 19 & -49 & -4 & $3.94 \mathrm{E}-7$ & $1.90 \mathrm{E}-6$ & $>$ & $1.00 \mathrm{E}-4$ \\
\hline HCT-15 & 0.355 & 2.654 & 2.541 & 2.436 & 0.901 & 0.250 & 0.147 & 95 & 90 & 24 & -30 & -59 & $4.04 \mathrm{E}-7$ & $2.79 \mathrm{E}-6$ & & $5.06 \mathrm{E}-5$ \\
\hline HT29 & 0.240 & 2.091 & 2.059 & 2.184 & 0.550 & 0.299 & 0.326 & 98 & 105 & 17 & 3 & 5 & $4.20 \mathrm{E}-7$ & $>1.00 \mathrm{E}-4$ & $>$ & $1.00 \mathrm{E}-4$ \\
\hline KM12 & 0.467 & 3.002 & 3.038 & 2.858 & 1.141 & 0.422 & 0.407 & 101 & 94 & 27 & -10 & -13 & $4.51 \mathrm{E}-7$ & $5.39 \mathrm{E}-6$ & $>$ & $1.00 \mathrm{E}-4$ \\
\hline SW-620 & 0.200 & 1.511 & 1.465 & 1.345 & 0.486 & 0.250 & 0.211 & 96 & 87 & 22 & 4 & 1 & $3.71 \mathrm{E}-7$ & $>1.00 \mathrm{E}-4$ & $>$ & $1.00 \mathrm{E}-4$ \\
\hline CNS Cancer & & & & & & & & & & & & & & & & \\
\hline SF-268 & 0.759 & 2.591 & 2.480 & 2.417 & 1.686 & 0.371 & 0.552 & 94 & 90 & 51 & -51 & -27 & $1.01 \mathrm{E}-6$ & $3.14 \mathrm{E}-6$ & & \\
\hline SF-295 & 0.484 & 1.792 & 1.649 & 1.574 & 0.605 & 0.109 & 0.195 & 89 & 83 & 9 & -77 & -60 & $2.82 \mathrm{E}-7$ & $1.28 \mathrm{E}-6$ & & $4.82 \mathrm{E}-6$ \\
\hline SF-539 & 0.884 & 2.800 & 2.709 & 2.639 & 1.280 & 0.084 & 0.187 & 95 & 92 & 21 & -90 & -79 & $3.86 \mathrm{E}-7$ & $1.53 \mathrm{E}-6$ & & 4.32E-6 \\
\hline SNB-19 & 0.749 & 2.713 & 2.532 & 2.510 & 1.385 & 0.349 & 0.411 & 91 & 90 & 32 & -53 & -45 & $4.93 \mathrm{E}-7$ & $2.38 \mathrm{E}-6$ & & \\
\hline SNB-75 & 0.890 & 1.736 & 1.590 & 1.512 & 0.984 & 0.564 & 0.385 & 83 & 73 & 11 & -37 & -57 & $2.38 \mathrm{E}-7$ & $1.70 \mathrm{E}-6$ & & $4.59 \mathrm{E}-5$ \\
\hline U251 & 0.256 & 1.717 & 1.711 & 1.569 & 0.703 & 0.138 & 0.318 & 100 & 90 & 31 & -46 & 4 & $4.70 \mathrm{E}-7$ & & $>$ & $1.00 \mathrm{E}-4$ \\
\hline Melanoma & & & & & & & & & & & & & & & & \\
\hline LOX IMVI & 0.406 & 3.048 & 2.995 & 2.890 & 1.139 & 0.007 & 0.198 & 98 & 94 & 28 & -98 & -51 & $4.61 \mathrm{E}-7$ & $1.66 \mathrm{E}-6$ & & 4.14E-6 \\
\hline MALME-3M & 0.679 & 1.767 & 1.679 & 1.582 & 1.111 & 0.469 & 0.221 & 92 & 83 & 40 & -31 & -67 & $5.78 \mathrm{E}-7$ & $3.65 \mathrm{E}-6$ & & 3.33E-5 \\
\hline M14 & 0.388 & 1.782 & 1.733 & 1.745 & 0.891 & 0.258 & 0.334 & 97 & 97 & 36 & -34 & -14 & $5.93 \mathrm{E}-7$ & $3.29 \mathrm{E}-6$ & $>$ & $1.00 \mathrm{E}-4$ \\
\hline MDA-MB-435 & 0.443 & 2.239 & 2.146 & 1.721 & 0.315 & 0.124 & 0.208 & 95 & 71 & -29 & -72 & -53 & $1.63 \mathrm{E}-7$ & $5.14 \mathrm{E}-7$ & & $3.08 \mathrm{E}-6$ \\
\hline SK-MEL-2 & 1.315 & 3.012 & 2.958 & 2.952 & 1.980 & 0.713 & 0.722 & 97 & 96 & 39 & -46 & -45 & $6.47 \mathrm{E}-7$ & $2.89 \mathrm{E}-6$ & $>$ & $1.00 \mathrm{E}-4$ \\
\hline SK-MEL-28 & 0.607 & 2.349 & 2.196 & 2.138 & 1.395 & 0.129 & 0.147 & 91 & 88 & 45 & -79 & -76 & $7.72 \mathrm{E}-7$ & $2.31 \mathrm{E}-6$ & & 5.86E-6 \\
\hline SK-MEL-5 & 0.752 & 3.259 & 3.210 & 3.145 & 1.332 & 0.017 & 0.372 & 98 & 95 & 23 & -98 & -51 & 4.25E-7 & $1.55 \mathrm{E}-6$ & & 4.02E-6 \\
\hline UACC-257 & 1.097 & 2.828 & 2.729 & 2.570 & 2.077 & 1.032 & 0.712 & 94 & 85 & 57 & -6 & -35 & $1.28 \mathrm{E}-6$ & $8.03 \mathrm{E}-6$ & $>$ & $1.00 \mathrm{E}-4$ \\
\hline UACC-62 & 1.057 & 3.157 & 3.091 & 2.979 & 1.695 & 0.035 & 0.243 & 97 & 91 & 30 & -97 & -77 & $4.77 \mathrm{E}-7$ & $1.73 \mathrm{E}-6$ & & $4.29 \mathrm{E}-6$ \\
\hline Ovarian Canc & & & & & & & & & & & & & & & & \\
\hline IGROV1 & 0.358 & 1.921 & 1.869 & 1.796 & 0.900 & 0.229 & 0.151 & 97 & 92 & 35 & -36 & -58 & $5.40 \mathrm{E}-7$ & $3.09 \mathrm{E}-6$ & & $4.38 \mathrm{E}-5$ \\
\hline OVCAR-3 & 0.547 & 2.171 & 2.185 & 2.149 & 0.725 & 0.252 & 0.330 & 101 & 99 & 11 & -54 & -40 & $3.59 \mathrm{E}-7$ & $1.47 \mathrm{E}-6$ & & \\
\hline OVCAR-4 & 0.555 & 1.319 & 1.293 & 1.250 & 1.034 & 0.502 & 0.287 & 97 & 91 & 63 & -10 & -48 & $1.50 \mathrm{E}-6$ & 7.37E-6 & $>$ & $1.00 \mathrm{E}-4$ \\
\hline OVCAR-5 & 0.528 & 1.766 & 1.661 & 1.581 & 1.223 & 0.385 & 0.102 & 92 & 85 & 56 & -27 & -81 & $1.18 \mathrm{E}-6$ & 4.73E-6 & & $2.67 \mathrm{E}-5$ \\
\hline OVCAR-8 & 0.418 & 2.349 & 2.277 & 2.236 & 1.528 & 0.512 & 0.470 & 96 & 94 & 57 & 5 & 3 & $1.39 \mathrm{E}-6$ & $>1.00 \mathrm{E}-4$ & $>$ & $1.00 \mathrm{E}-4$ \\
\hline NCI/ADR-RES & 0.621 & 2.263 & 2.239 & 2.116 & 0.928 & 0.369 & 0.223 & 99 & 91 & 19 & -41 & -64 & 3.69E-7 & $2.06 \mathrm{E}-6$ & & $2.50 \mathrm{E}-5$ \\
\hline SK-OV-3 & 0.927 & 2.389 & 2.278 & 2.249 & 1.586 & 0.700 & 0.426 & 92 & 90 & 45 & -24 & -54 & 7.77E-7 & $4.44 \mathrm{E}-6$ & & $7.27 \mathrm{E}-5$ \\
\hline Renal Cancer & & & & & & & & & & & & & & & & \\
\hline $786-0$ & 0.623 & 2.690 & 2.686 & 2.497 & 1.511 & 0.224 & 0.326 & 100 & 91 & 43 & -64 & -48 & $7.12 \mathrm{E}-7$ & $2.52 \mathrm{E}-6$ & & \\
\hline A498 & 1.828 & 2.812 & 2.664 & 2.603 & 1.943 & 0.094 & 0.254 & 85 & 79 & 12 & -95 & -86 & $2.68 \mathrm{E}-7$ & 1.29E-6 & & $3.79 \mathrm{E}-6$ \\
\hline $\mathrm{ACHN}$ & 0.320 & 1.693 & 1.692 & 1.597 & 0.819 & 0.240 & 0.040 & 100 & 93 & 36 & -25 & -88 & $5.74 \mathrm{E}-7$ & $3.90 \mathrm{E}-6$ & & $2.50 \mathrm{E}-5$ \\
\hline CAKI-1 & 0.613 & 2.481 & 2.295 & 2.176 & 1.138 & 0.521 & 0.200 & 90 & 84 & 28 & -15 & -67 & $4.04 \mathrm{E}-7$ & $4.47 \mathrm{E}-6$ & & 4.65E-5 \\
\hline RXF 393 & 1.197 & 1.469 & 1.421 & 1.350 & 1.007 & 0.236 & 0.267 & 82 & 56 & -16 & -80 & -78 & $1.22 \mathrm{E}-7$ & $6.02 \mathrm{E}-7$ & & $3.39 \mathrm{E}-6$ \\
\hline SN12C & 0.664 & 2.655 & 2.498 & 2.490 & 1.549 & 0.093 & 0.092 & 92 & 92 & 44 & -86 & -86 & $7.64 \mathrm{E}-7$ & $2.19 \mathrm{E}-6$ & & $5.30 \mathrm{E}-6$ \\
\hline TK-10 & 0.733 & 2.248 & 2.224 & 2.153 & 1.758 & 0.724 & 0.502 & 98 & 94 & 68 & -1 & -32 & $1.80 \mathrm{E}-6$ & $9.58 \mathrm{E}-6$ & $>$ & $1.00 \mathrm{E}-4$ \\
\hline UO-31 & 0.630 & 2.030 & 1.781 & 1.745 & 1.207 & 0.444 & 0.136 & 82 & 80 & 41 & -30 & -78 & $5.90 \mathrm{E}-7$ & $3.82 \mathrm{E}-6$ & & $2.62 \mathrm{E}-5$ \\
\hline Prostate Cancer & & & & & & & & & & & & & & & & \\
\hline $\mathrm{PC}-3$ & 0.511 & 2.292 & 2.259 & 2.285 & 1.154 & 0.468 & 0.375 & 98 & 100 & 36 & -9 & -27 & $6.04 \mathrm{E}-7$ & $6.44 \mathrm{E}-6$ & $>$ & $1.00 \mathrm{E}-4$ \\
\hline DU-145 & 0.346 & 1.770 & 1.784 & 1.708 & 1.084 & 0.356 & 0.193 & 101 & 96 & 52 & 1 & -44 & $1.08 \mathrm{E}-6$ & $1.04 \mathrm{E}-5$ & $>$ & $1.00 \mathrm{E}-4$ \\
\hline Breast Cancer & & & & & & & & & & & & & & & & \\
\hline MCF7 & 0.309 & 1.813 & 1.665 & 1.640 & 0.498 & 0.259 & 0.175 & 90 & 88 & 13 & -16 & -43 & $3.21 \mathrm{E}-7$ & $2.74 \mathrm{E}-6$ & $>$ & $1.00 \mathrm{E}-4$ \\
\hline MDA-MB-231/ATCC & 0.656 & 1.658 & 1.650 & 1.591 & 1.261 & 0.144 & 0.152 & 99 & 93 & 60 & -78 & -77 & $1.19 \mathrm{E}-6$ & $2.73 \mathrm{E}-6$ & & $6.27 \mathrm{E}-6$ \\
\hline HS 578T & 0.915 & 2.037 & 1.983 & 1.977 & 1.195 & 0.720 & 0.629 & 95 & 95 & 25 & -21 & -31 & 4.37E-7 & $3.46 \mathrm{E}-6$ & $>$ & $1.00 \mathrm{E}-4$ \\
\hline BT-549 & 1.174 & 2.572 & 2.520 & 2.438 & 1.774 & 0.481 & 0.652 & 96 & 90 & 43 & -59 & -45 & $7.08 \mathrm{E}-7$ & $2.63 \mathrm{E}-6$ & & \\
\hline T-47D & 1.075 & 2.781 & 2.657 & 2.561 & 1.669 & 1.155 & 0.700 & 93 & 87 & 35 & 5 & -35 & $5.12 \mathrm{E}-7$ & $1.31 \mathrm{E}-5$ & $>$ & $1.00 \mathrm{E}-4$ \\
\hline MDA-MB-468 & 0.915 & 1.680 & 1.642 & 1.560 & 1.074 & 0.757 & 0.578 & 95 & 84 & 21 & -17 & -37 & $3.46 \mathrm{E}-7$ & $3.51 \mathrm{E}-6$ & $>$ & $1.00 \mathrm{E}-4$ \\
\hline
\end{tabular}


In-Vitro Testing Results

\begin{tabular}{|l|l|l|l|}
\hline NSC : D - 810517 /1 & Experiment ID : 1901NS56 & Test Type : 08 & Units : Molar \\
\hline Report Date : February 24, 2019 & Test Date : January 28, 2019 & QNS : & MC : \\
\hline COMI : 2351731874 & Stain Reagent : SRB Dual-Pass Related & SSPL : OM6Y & \\
\hline
\end{tabular}

\begin{tabular}{|c|c|c|c|c|c|c|c|c|c|c|c|c|c|c|c|c|}
\hline \multirow[b]{3}{*}{ Panel/Cell Line } & \multicolumn{13}{|c|}{ Log10 Concentration } & \multirow{3}{*}{ TGI } & \multirow{3}{*}{\multicolumn{2}{|c|}{ LC50 }} \\
\hline & \multirow{2}{*}{$\begin{array}{l}\text { Time } \\
\text { Zero }\end{array}$} & & & Mear & Optical & Densitie & & & & reent & rowth & & & & & \\
\hline & & Ctrl & -8.0 & -7.0 & -6.0 & -5.0 & -4.0 & -8.0 & -7.0 & -6.0 & -5.0 & -4.0 & GI50 & & & \\
\hline Leukemia & & & & & & & & & & & & & & & & \\
\hline CCRF-CEM & 0.580 & 2.988 & 2.951 & 2.994 & 2.038 & 0.859 & 0.692 & 98 & 100 & 61 & 12 & 5 & $1.64 \mathrm{E}-6$ & $>1.00 \mathrm{E}-4$ & $>$ & $1.00 \mathrm{E}-4$ \\
\hline HL-60(TB) & 0.877 & 3.287 & 3.194 & 3.255 & 1.333 & 0.847 & 0.582 & 96 & 99 & 19 & -3 & -34 & 4.07E-7 & 6.99E-6 & $>$ & $1.00 \mathrm{E}-4$ \\
\hline $\mathrm{K}-562$ & 0.214 & 2.718 & 2.623 & 2.611 & 0.689 & 0.480 & 0.325 & 96 & 96 & 19 & 11 & 4 & $3.94 \mathrm{E}-7$ & $>1.00 \mathrm{E}-4$ & $>$ & $1.00 \mathrm{E}-4$ \\
\hline MOLT-4 & 0.550 & 2.961 & 2.934 & 2.948 & 1.681 & 0.959 & 0.594 & 99 & 99 & 47 & 17 & 2 & $8.73 \mathrm{E}-7$ & $>1.00 \mathrm{E}-4$ & $>$ & $1.00 \mathrm{E}-4$ \\
\hline RPMI-8226 & 1.128 & 3.103 & 3.062 & 3.086 & 2.772 & 1.466 & 0.832 & 98 & 99 & 83 & 17 & -26 & $3.18 \mathrm{E}-6$ & $2.48 \mathrm{E}-5$ & $>$ & $1.00 \mathrm{E}-4$ \\
\hline SR & 0.281 & 1.715 & 1.512 & 1.537 & 0.602 & 0.512 & 0.396 & 86 & 88 & 22 & 16 & 8 & 3.77E-7 & $>1.00 \mathrm{E}-4$ & $>$ & $1.00 \mathrm{E}-4$ \\
\hline Non-Small Cell Lung & Cancer & & & & & & & & & & & & & & & \\
\hline A549/ATCC & 0.373 & 2.432 & 2.192 & 2.236 & 1.617 & 0.728 & 0.528 & 88 & 90 & 60 & 17 & 8 & $1.74 \mathrm{E}-6$ & $>1.00 \mathrm{E}-4$ & $>$ & $1.00 \mathrm{E}-4$ \\
\hline EKVX & 0.679 & 1.996 & 1.860 & 1.889 & 1.584 & 1.025 & 0.628 & 90 & 92 & 69 & 26 & -8 & $2.76 \mathrm{E}-6$ & $5.99 \mathrm{E}-5$ & $>$ & $1.00 \mathrm{E}-4$ \\
\hline HOP-62 & 0.451 & 1.954 & 1.810 & 1.870 & 1.265 & 0.711 & 0.196 & 90 & 94 & 54 & 17 & -57 & $1.30 \mathrm{E}-6$ & $1.71 \mathrm{E}-5$ & & $8.15 \mathrm{E}-5$ \\
\hline HOP-92 & 1.205 & 1.968 & 1.838 & 1.880 & 1.752 & 1.460 & 0.786 & 83 & 88 & 72 & 33 & -35 & $3.68 \mathrm{E}-6$ & 3.09E-5 & $>$ & $1.00 \mathrm{E}-4$ \\
\hline $\mathrm{NCl}-\mathrm{H} 226$ & 1.465 & 3.116 & 3.059 & 3.102 & 2.832 & 1.932 & 0.545 & 97 & 99 & 83 & 28 & -63 & 3.99E-6 & $2.04 \mathrm{E}-5$ & & 7.23E-5 \\
\hline $\mathrm{NCl}-\mathrm{H} 23$ & 0.538 & 1.634 & 1.587 & 1.623 & 1.401 & 0.806 & 0.296 & 96 & 99 & 79 & 24 & -45 & $3.38 \mathrm{E}-6$ & $2.25 \mathrm{E}-5$ & $>$ & $1.00 \mathrm{E}-4$ \\
\hline $\mathrm{NCl}-\mathrm{H} 322 \mathrm{M}$ & 0.694 & 2.032 & 1.965 & 2.035 & 1.943 & 1.185 & 0.704 & 95 & 100 & 93 & 37 & 1 & $5.81 \mathrm{E}-6$ & $>1.00 \mathrm{E}-4$ & $>$ & $1.00 \mathrm{E}-4$ \\
\hline $\mathrm{NCl}-\mathrm{H} 460$ & 0.284 & 2.989 & 3.088 & 3.029 & 1.168 & 0.354 & 0.096 & 104 & 101 & 33 & 3 & -66 & $5.60 \mathrm{E}-7$ & 1.09E-5 & & $5.79 \mathrm{E}-5$ \\
\hline $\mathrm{NCl}-\mathrm{H} 522$ & 0.911 & 2.816 & 2.531 & 2.551 & 1.774 & 0.999 & 0.695 & 85 & 86 & 45 & 5 & -24 & $7.66 \mathrm{E}-7$ & $1.45 \mathrm{E}-5$ & $>$ & $1.00 \mathrm{E}-4$ \\
\hline Colon Cancer & & & & & & & & & & & & & & & & \\
\hline COLO 205 & 0.443 & 2.044 & 2.067 & 2.067 & 1.527 & 0.432 & 0.124 & 101 & 101 & 68 & -2 & -72 & $1.79 \mathrm{E}-6$ & $9.22 \mathrm{E}-6$ & & $4.82 \mathrm{E}-5$ \\
\hline HCC-2998 & 0.652 & 2.493 & 2.430 & 2.476 & 1.828 & 0.537 & 0.154 & 97 & 99 & 64 & -18 & -76 & $1.48 \mathrm{E}-6$ & $6.07 \mathrm{E}-6$ & & 3.55E-5 \\
\hline НСТ-116 & 0.242 & 2.460 & 2.339 & 2.455 & 1.190 & 0.398 & 0.096 & 95 & 100 & 43 & 7 & -60 & $7.46 \mathrm{E}-7$ & $1.27 \mathrm{E}-5$ & & $7.02 \mathrm{E}-5$ \\
\hline HCT-15 & 0.355 & 2.648 & 2.551 & 2.521 & 1.154 & 0.603 & 0.258 & 96 & 94 & 35 & 11 & -27 & $5.57 \mathrm{E}-7$ & $1.92 \mathrm{E}-5$ & $>$ & $1.00 \mathrm{E}-4$ \\
\hline HT29 & 0.240 & 2.155 & 2.023 & 2.110 & 0.668 & 0.371 & 0.190 & 93 & 98 & 22 & 7 & -21 & 4.29E-7 & $1.76 \mathrm{E}-5$ & $>$ & $1.00 \mathrm{E}-4$ \\
\hline KM12 & 0.467 & 2.917 & 2.818 & 2.861 & 1.390 & 0.782 & 0.168 & 96 & 98 & 38 & 13 & -64 & $6.24 \mathrm{E}-7$ & $1.47 \mathrm{E}-5$ & & $6.57 \mathrm{E}-5$ \\
\hline SW-620 & 0.200 & 1.460 & 1.439 & 1.383 & 0.557 & 0.388 & 0.117 & 98 & 94 & 28 & 15 & -42 & $4.68 \mathrm{E}-7$ & $1.83 \mathrm{E}-5$ & $>$ & $1.00 \mathrm{E}-4$ \\
\hline CNS Cancer & & & & & & & & & & & & & & & & \\
\hline SF-268 & 0.759 & 2.525 & 2.362 & 2.392 & 2.060 & 1.280 & 0.407 & 91 & 92 & 74 & 30 & -46 & $3.44 \mathrm{E}-6$ & $2.45 \mathrm{E}-5$ & $>$ & $1.00 \mathrm{E}-4$ \\
\hline SF-295 & 0.484 & 1.786 & 1.599 & 1.674 & 0.851 & 0.436 & 0.073 & 86 & 91 & 28 & -10 & -85 & $4.52 \mathrm{E}-7$ & $5.46 \mathrm{E}-6$ & & $3.41 \mathrm{E}-5$ \\
\hline SF-539 & 0.884 & 2.838 & 2.666 & 2.785 & 1.992 & 0.541 & 0.073 & 91 & 97 & 57 & -39 & -92 & $1.17 \mathrm{E}-6$ & $3.92 \mathrm{E}-6$ & & 1.62E-5 \\
\hline SNB-19 & 0.749 & 2.710 & 2.546 & 2.568 & 1.840 & 1.202 & 0.763 & 92 & 93 & 56 & 23 & 1 & $1.49 \mathrm{E}-6$ & $>1.00 \mathrm{E}-4$ & $>$ & $1.00 \mathrm{E}-4$ \\
\hline SNB-75 & 0.890 & 1.782 & 1.572 & 1.622 & 1.260 & 0.788 & 0.564 & 77 & 82 & 41 & -11 & -37 & $6.16 \mathrm{E}-7$ & $6.07 \mathrm{E}-6$ & $>$ & $1.00 \mathrm{E}-4$ \\
\hline U251 & 0.256 & 1.770 & 1.686 & 1.645 & 1.074 & 0.440 & 0.129 & 94 & 92 & 54 & 12 & -50 & $1.25 \mathrm{E}-6$ & $1.57 \mathrm{E}-5$ & $>$ & $1.00 \mathrm{E}-4$ \\
\hline Melanoma & & & & & & & & & & & & & & & & \\
\hline LOX IMVI & 0.406 & 3.089 & 2.907 & 3.026 & 1.540 & 1.184 & 0.018 & 93 & 98 & 42 & 29 & -96 & $7.25 \mathrm{E}-7$ & $1.71 \mathrm{E}-5$ & & 4.30E-5 \\
\hline MALME-3M & 0.679 & 1.790 & 1.647 & 1.682 & 1.235 & 1.145 & 0.216 & 87 & 90 & 50 & 42 & -68 & $9.99 \mathrm{E}-7$ & $2.40 \mathrm{E}-5$ & & $6.84 \mathrm{E}-5$ \\
\hline M14 & 0.388 & 1.815 & 1.724 & 1.760 & 0.982 & 0.582 & 0.136 & 94 & 96 & 42 & 14 & -65 & $7.01 \mathrm{E}-7$ & $1.49 \mathrm{E}-5$ & & $6.43 \mathrm{E}-5$ \\
\hline MDA-MB-435 & 0.443 & 2.290 & 2.144 & 2.048 & 0.333 & 0.189 & 0.056 & 92 & 87 & -25 & -57 & -87 & $2.14 \mathrm{E}-7$ & $5.98 \mathrm{E}-7$ & & $5.94 \mathrm{E}-6$ \\
\hline SK-MEL-2 & 1.315 & 3.049 & 2.907 & 2.913 & 2.244 & 1.847 & 0.434 & 92 & 92 & 54 & 31 & -67 & $1.43 \mathrm{E}-6$ & $2.06 \mathrm{E}-5$ & & $6.70 \mathrm{E}-5$ \\
\hline SK-MEL-28 & 0.607 & 2.304 & 2.229 & 2.234 & 1.698 & 1.342 & 0.041 & 96 & 96 & 64 & 43 & -93 & $4.80 \mathrm{E}-6$ & $2.08 \mathrm{E}-5$ & & 4.82E-5 \\
\hline SK-MEL-5 & 0.752 & 3.224 & 3.156 & 3.167 & 1.884 & 1.040 & -0.012 & 97 & 98 & 46 & 12 & -100 & $8.29 \mathrm{E}-7$ & $1.27 \mathrm{E}-5$ & & 3.57E-5 \\
\hline UACC-257 & 1.097 & 2.761 & 2.659 & 2.596 & 2.297 & 1.959 & 0.524 & 94 & 90 & 72 & 52 & -52 & $1.04 \mathrm{E}-5$ & $3.15 \mathrm{E}-5$ & & $9.52 \mathrm{E}-5$ \\
\hline UACC-62 & 1.057 & 3.153 & 3.112 & 3.070 & 2.066 & 1.448 & 0.036 & 98 & 96 & 48 & 19 & -97 & $9.14 \mathrm{E}-7$ & $1.45 \mathrm{E}-5$ & & $3.94 \mathrm{E}-5$ \\
\hline Ovarian Canc & & & & & & & & & & & & & & & & \\
\hline IGROV1 & 0.358 & 1.959 & 1.914 & 1.977 & 1.180 & 0.516 & 0.150 & 97 & 101 & 51 & 10 & -58 & $1.08 \mathrm{E}-6$ & $1.40 \mathrm{E}-5$ & & $7.57 \mathrm{E}-5$ \\
\hline OVCAR-3 & 0.547 & 2.074 & 2.031 & 2.112 & 0.857 & 0.506 & 0.077 & 97 & 102 & 20 & -7 & -86 & 4.35E-7 & $5.37 \mathrm{E}-6$ & & $3.48 \mathrm{E}-5$ \\
\hline OVCAR-4 & 0.555 & 1.349 & 1.291 & 1.334 & 1.149 & 0.761 & 0.540 & 93 & 98 & 75 & 26 & -3 & $3.22 \mathrm{E}-6$ & $7.99 \mathrm{E}-5$ & $>$ & $1.00 \mathrm{E}-4$ \\
\hline OVCAR-5 & 0.528 & 1.766 & 1.678 & 1.704 & 1.541 & 0.717 & 0.390 & 93 & 95 & 82 & 15 & -26 & $3.01 \mathrm{E}-6$ & $2.34 \mathrm{E}-5$ & $>$ & $1.00 \mathrm{E}-4$ \\
\hline OVCAR-8 & 0.418 & 2.355 & 2.291 & 2.346 & 1.898 & 0.784 & 0.402 & 97 & 100 & 76 & 19 & -4 & $2.88 \mathrm{E}-6$ & $6.71 \mathrm{E}-5$ & $>$ & $1.00 \mathrm{E}-4$ \\
\hline NCI/ADR-RES & 0.621 & 2.320 & 2.242 & 2.302 & 1.327 & 0.542 & 0.344 & 95 & 99 & 42 & -13 & -45 & $7.13 \mathrm{E}-7$ & $5.81 \mathrm{E}-6$ & $>$ & $1.00 \mathrm{E}-4$ \\
\hline SK-OV-3 & 0.927 & 2.350 & 2.159 & 2.198 & 1.742 & 1.191 & 0.762 & 87 & 89 & 57 & 19 & -18 & $1.54 \mathrm{E}-6$ & $3.23 E-5$ & $>$ & $1.00 \mathrm{E}-4$ \\
\hline Renal Cancer & & & & & & & & & & & & & & & & \\
\hline $786-0$ & 0.623 & 2.741 & 2.611 & 2.688 & 2.262 & 1.083 & 0.259 & 94 & 97 & 77 & 22 & -59 & $3.10 \mathrm{E}-6$ & $1.87 \mathrm{E}-5$ & & 7.83E-5 \\
\hline A498 & 1.828 & 2.817 & 2.786 & 2.817 & 2.074 & 1.654 & 0.363 & 97 & 100 & 25 & -10 & -80 & 4.62E-7 & $5.28 \mathrm{E}-6$ & & $3.74 \mathrm{E}-5$ \\
\hline $\mathrm{ACHN}$ & 0.320 & 1.687 & 1.636 & 1.696 & 1.257 & 0.650 & 0.275 & 96 & 101 & 69 & 24 & -14 & $2.61 \mathrm{E}-6$ & $4.28 \mathrm{E}-5$ & $>$ & $1.00 \mathrm{E}-4$ \\
\hline CAKI-1 & 0.613 & 2.528 & 2.333 & 2.328 & 1.293 & 0.987 & 0.527 & 90 & 90 & 35 & 20 & -14 & $5.39 \mathrm{E}-7$ & $3.80 \mathrm{E}-5$ & $>$ & $1.00 \mathrm{E}-4$ \\
\hline RXF 393 & 1.197 & 1.650 & 1.615 & 1.619 & 1.361 & 0.900 & 0.146 & 92 & 93 & 36 & -25 & -88 & $5.73 \mathrm{E}-7$ & $3.92 \mathrm{E}-6$ & & $2.51 \mathrm{E}-5$ \\
\hline SN12C & 0.664 & 2.684 & 2.492 & 2.596 & 2.280 & 1.213 & 0.086 & 90 & 96 & 80 & 27 & -87 & 3.69E-6 & $1.73 \mathrm{E}-5$ & & 4.73E-5 \\
\hline TK-10 & 0.733 & 2.329 & 2.247 & 2.212 & 1.998 & 1.387 & 0.745 & 95 & 93 & 79 & 41 & 1 & $5.81 \mathrm{E}-6$ & $>1.00 \mathrm{E}-4$ & $>$ & $1.00 \mathrm{E}-4$ \\
\hline บo-31 & 0.630 & 2.026 & 1.827 & 1.843 & 1.445 & 0.925 & 0.280 & 86 & 87 & 58 & 21 & -56 & $1.68 \mathrm{E}-6$ & $1.89 \mathrm{E}-5$ & & $8.45 \mathrm{E}-5$ \\
\hline Prostate Cancer & & & & & & & & & & & & & & & & \\
\hline $\mathrm{PC}-3$ & 0.511 & 2.297 & 2.205 & 2.223 & 1.605 & 0.725 & 0.355 & 95 & 96 & 61 & 12 & -31 & $1.69 \mathrm{E}-6$ & $1.91 \mathrm{E}-5$ & $>$ & $1.00 \mathrm{E}-4$ \\
\hline DU-145 & 0.346 & 1.708 & 1.708 & 1.767 & 1.470 & 0.531 & 0.242 & 100 & 104 & 83 & 14 & -30 & $2.96 \mathrm{E}-6$ & $2.04 \mathrm{E}-5$ & $>$ & $1.00 \mathrm{E}-4$ \\
\hline Breast Cancer & & & & & & & & & & & & & & & & \\
\hline MCF7 & 0.309 & 1.881 & 1.815 & 1.864 & 0.733 & 0.468 & 0.262 & 96 & 99 & 27 & 10 & -15 & $4.78 \mathrm{E}-7$ & $2.50 \mathrm{E}-5$ & $>$ & $1.00 \mathrm{E}-4$ \\
\hline MDA-MB-231/ATCC & 0.656 & 1.643 & 1.649 & 1.616 & 1.456 & 0.772 & 0.226 & 101 & 97 & 81 & 12 & -66 & $2.81 \mathrm{E}-6$ & $1.42 \mathrm{E}-5$ & & $6.28 \mathrm{E}-5$ \\
\hline HS 578T & 0.915 & 1.990 & 1.955 & 1.906 & 1.407 & 0.953 & 0.684 & 97 & 92 & 46 & 3 & -25 & $8.11 \mathrm{E}-7$ & $1.32 \mathrm{E}-5$ & $>$ & $1.00 \mathrm{E}-4$ \\
\hline BT-549 & 1.174 & 2.627 & 2.481 & 2.562 & 2.100 & 1.655 & 0.200 & 90 & 96 & 64 & 33 & -83 & $2.81 \mathrm{E}-6$ & $1.93 \mathrm{E}-5$ & & $5.20 \mathrm{E}-5$ \\
\hline T-47D & 1.075 & 2.755 & 2.544 & 2.518 & 1.916 & 1.433 & 1.005 & 87 & 86 & 50 & 21 & -7 & $1.01 \mathrm{E}-6$ & $5.83 \mathrm{E}-5$ & $>$ & $1.00 \mathrm{E}-4$ \\
\hline MDA-MB-468 & 0.915 & 1.548 & 1.455 & 1.513 & 0.967 & 0.949 & 0.356 & 85 & 94 & 8 & 5 & -61 & 3.27E-7 & $1.20 \mathrm{E}-5$ & & $6.81 \mathrm{E}-5$ \\
\hline
\end{tabular}


In-Vitro Testing Results

\begin{tabular}{|l|l|l|l|}
\hline NSC : D - 810511/1 & Experiment ID : 1901NS52 & Test Type : 08 & Units : Molar \\
\hline Report Date : February 02, 2019 & Test Date : January 14, 2019 & QNS : & MC : \\
\hline COMI : 2351801054 & Stain Reagent : SRB Dual-Pass Related & SSPL : OM6Y & \\
\hline
\end{tabular}

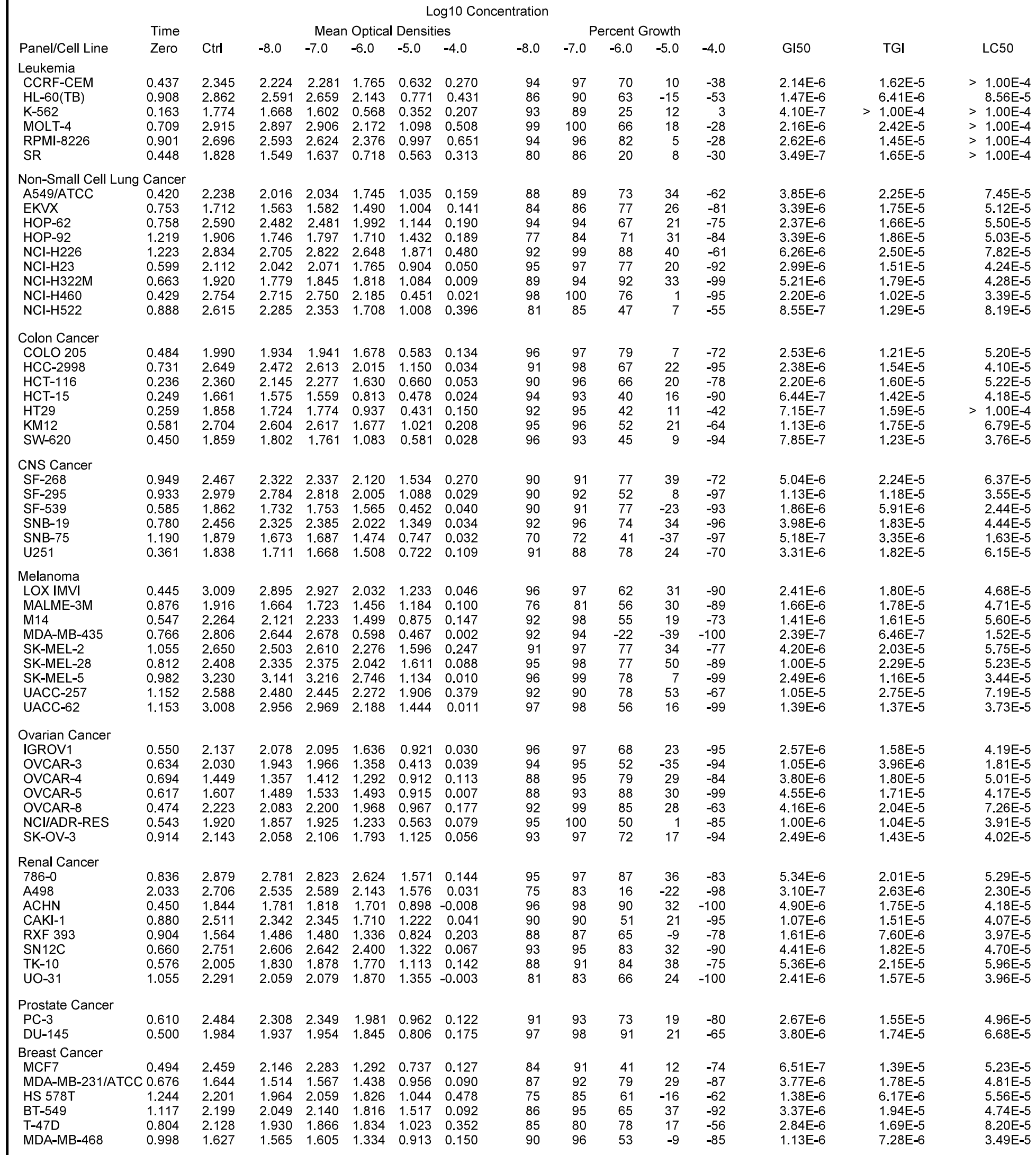


In-Vitro Testing Results

\begin{tabular}{|l|l|l|l|}
\hline NSC : D - 816475/1 & Experiment ID : 1910RS70 & Test Type : 08 & Units : Molar \\
\hline Report Date : October 31, 2019 & Test Date : October 07, 2019 & QNS : & MC : \\
\hline COMI : 2351710830 & Stain Reagent : SRB Dual-Pass Related & SSPL : OM6Y & \\
\hline
\end{tabular}

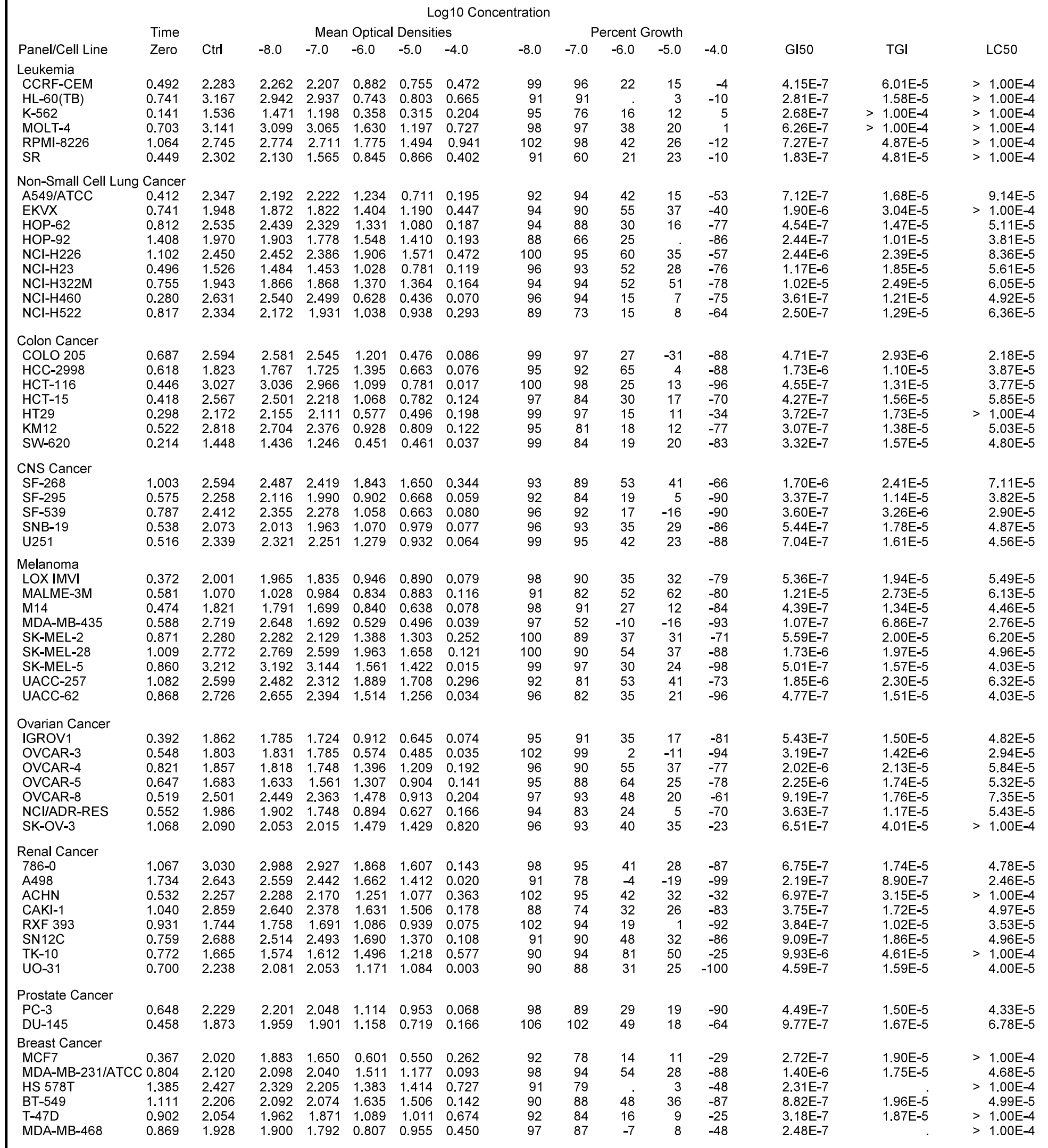


In-Vitro Testing Results

\begin{tabular}{|l|l|l|l|}
\hline NSC : D - 823369/1 & Experiment ID : 2008NS40 & Test Type : 08 & Units : Molar \\
\hline Report Date : March 24, 2021 & Test Date : August 24, 2020 & QNS : & MC : \\
\hline COMI : KLB1-112 & Stain Reagent : SRB Dual-Pass Related & SSPL : 1C1X & \\
\hline
\end{tabular}

Log10 Concentration

\begin{tabular}{|c|c|c|c|c|c|c|c|c|c|c|c|c|c|c|c|}
\hline \multirow[b]{2}{*}{ Panel/Cell Line } & \multirow{2}{*}{$\begin{array}{l}\text { Time } \\
\text { Zero }\end{array}$} & \multirow[b]{2}{*}{ Ctrl } & \multicolumn{5}{|c|}{ Mean Optical Densities } & \multicolumn{5}{|c|}{ Percent Growth } & \multirow[b]{2}{*}{ GI50 } & & \\
\hline & & & -8.0 & -7.0 & -6.0 & -5.0 & -4.0 & -8.0 & -7.0 & -6.0 & -5.0 & -4.0 & & TGI & LC50 \\
\hline Leukemia & & & & & & & & & & & & & & & \\
\hline CCRF-CEM & 0.354 & 1.576 & 1.579 & 1.614 & 0.665 & 0.567 & 0.463 & 100 & 103 & 25 & 17 & 9 & 4.83E-7 & $>1.00 \mathrm{E}-4$ & $>1.00 \mathrm{E}-4$ \\
\hline HL-60(TB) & 0.732 & 2.664 & 2.520 & 2.842 & 0.869 & 0.863 & 0.522 & 93 & 109 & 7 & 7 & -29 & 3.80E-7 & 1.55E-5 & $>1.00 \mathrm{E}-4$ \\
\hline $\mathrm{K}-562$ & 0.198 & 1.912 & 1.920 & 1.831 & 0.554 & 0.450 & 0.322 & 100 & 95 & 21 & 15 & 7 & 4.05E-7 & $>1.00 \mathrm{E}-4$ & $>1.00 \mathrm{E}-4$ \\
\hline MOLT-4 & 0.633 & 2.918 & 2.923 & 2.978 & 1.595 & 1.068 & 0.738 & 100 & 103 & 42 & 19 & 5 & 7.40E-7 & $>1.00 \mathrm{E}-4$ & $>1.00 \mathrm{E}-4$ \\
\hline RPMI-8226 & 0.988 & 2.402 & 2.337 & 2.385 & 1.423 & 1.239 & 0.811 & 95 & 99 & 31 & 18 & -18 & $5.21 \mathrm{E}-7$ & 3.15E-5 & $>1.00 \mathrm{E}-4$ \\
\hline & 0.289 & 1.740 & 1.566 & 1.500 & 0.624 & 0.577 & 0.384 & 88 & 83 & 23 & 20 & 7 & 3.59E-7 & $>1.00 \mathrm{E}-4$ & $>1.00 \mathrm{E}-4$ \\
\hline Non-Small Cell Lung & Cancer & & & & & & & & & & & & & & \\
\hline A549/ATCC & 0.481 & 2.403 & 2.299 & 2.233 & 1.382 & 0.935 & 0.543 & 95 & 91 & 47 & 24 & 3 & $8.50 \mathrm{E}-7$ & $>1.00 \mathrm{E}-4$ & $>1.00 \mathrm{E}-4$ \\
\hline EKVX & 0.645 & 2.039 & 1.949 & 1.897 & 1.424 & 1.115 & 0.629 & 94 & 90 & 56 & 34 & -2 & $1.84 \mathrm{E}-6$ & $8.54 \mathrm{E}-5$ & $>1.00 \mathrm{E}-4$ \\
\hline HOP-62 & 1.021 & 2.491 & 2.378 & 2.338 & 1.742 & 1.569 & 0.914 & 92 & 90 & 49 & 37 & -10 & $9.48 \mathrm{E}-7$ & $6.03 \mathrm{E}-5$ & $>1.00 \mathrm{E}-4$ \\
\hline HOP-92 & 1.294 & 2.125 & 2.072 & 1.920 & 1.673 & 1.427 & 1.078 & 94 & 75 & 46 & 16 & -17 & 7.12E-7 & $3.08 \mathrm{E}-5$ & $>1.00 \mathrm{E}-4$ \\
\hline $\mathrm{NCl}-\mathrm{H} 226$ & 1.250 & 2.994 & 2.888 & 2.857 & 1.901 & 1.560 & 0.848 & 94 & 92 & 37 & 18 & -32 & $5.87 \mathrm{E}-7$ & 2.27E-5 & $>1.00 \mathrm{E}-4$ \\
\hline $\mathrm{NCl}-\mathrm{H} 23$ & 0.659 & 1.909 & 1.830 & 1.865 & 1.425 & 0.975 & 0.614 & 94 & 96 & 61 & 25 & -7 & $2.05 \mathrm{E}-6$ & $6.10 \mathrm{E}-5$ & $>1.00 \mathrm{E}-4$ \\
\hline $\mathrm{NCl}-\mathrm{H} 322 \mathrm{M}$ & 0.932 & 2.266 & 2.215 & 2.170 & 1.751 & 1.602 & 1.073 & 96 & 93 & 61 & 50 & 11 & $1.01 \mathrm{E}-5$ & $>1.00 \mathrm{E}-4$ & $>1.00 \mathrm{E}-4$ \\
\hline $\mathrm{NCl}-\mathrm{H} 460$ & 0.543 & 3.323 & 3.302 & 3.341 & 1.506 & 0.921 & 0.567 & 99 & 101 & 35 & 14 & 1 & $5.85 \mathrm{E}-7$ & $>1.00 \mathrm{E}-4$ & $>1.00 \mathrm{E}-4$ \\
\hline $\mathrm{NCl}-\mathrm{H} 522$ & 0.960 & 2.353 & 2.206 & 2.177 & 1.336 & 1.107 & 0.717 & 89 & 87 & 27 & 11 & -25 & $4.16 \mathrm{E}-7$ & $1.97 \mathrm{E}-5$ & $>1.00 \mathrm{E}-4$ \\
\hline Colon Cancer & & & & & & & & & & & & & & & \\
\hline COLO 205 & 0.420 & 1.438 & 1.486 & 1.414 & 0.946 & 0.413 & 0.158 & 105 & 98 & 52 & -2 & -62 & $1.08 \mathrm{E}-6$ & $9.31 \mathrm{E}-6$ & $6.25 \mathrm{E}-5$ \\
\hline HCC-2998 & 0.664 & 2.063 & 1.912 & 1.938 & 1.556 & 0.844 & 0.464 & 89 & 91 & 64 & 13 & -30 & $1.86 \mathrm{E}-6$ & $1.99 \mathrm{E}-5$ & $>1.00 \mathrm{E}-4$ \\
\hline HCT-116 & 0.188 & 2.267 & 2.208 & 2.026 & 0.678 & 0.504 & 0.263 & 97 & 88 & 24 & 15 & 4 & 3.91E-7 & $>1.00 \mathrm{E}-4$ & $>1.00 \mathrm{E}-4$ \\
\hline HCT-15 & 0.271 & 1.614 & 1.537 & 1.436 & 0.627 & 0.445 & 0.268 & 94 & 87 & 26 & 13 & -1 & 4.07E-7 & $8.11 \mathrm{E}-5$ & $>1.00 \mathrm{E}-4$ \\
\hline HT29 & 0.337 & 1.959 & 1.930 & 2.000 & 0.583 & 0.426 & 0.334 & 98 & 103 & 15 & 5 & -1 & 3.99E-7 & $6.93 \mathrm{E}-5$ & $>1.00 \mathrm{E}-4$ \\
\hline KM12 & 0.632 & 3.003 & 2.992 & 2.649 & 1.362 & 1.077 & 0.499 & 100 & 85 & 31 & 19 & -21 & $4.43 \mathrm{E}-7$ & $2.96 \mathrm{E}-5$ & $>1.00 \mathrm{E}-4$ \\
\hline SW-620 & 0.325 & 2.156 & 2.113 & 2.095 & 0.850 & 0.801 & 0.477 & 98 & 97 & 29 & 26 & 8 & 4.85E-7 & $>1.00 E-4$ & $>1.00 \mathrm{E}-4$ \\
\hline CNS Cancer & & & & & & & & & & & & & & & \\
\hline SF-268 & 0.965 & 2.460 & 2.385 & 2.361 & 1.788 & 1.577 & 0.832 & 95 & 93 & 55 & 41 & -14 & $2.28 \mathrm{E}-6$ & $5.59 \mathrm{E}-5$ & $>1.00 \mathrm{E}-4$ \\
\hline SF-295 & 0.871 & 2.922 & 2.750 & 2.684 & 1.382 & 1.033 & 0.415 & 92 & 88 & 25 & 8 & -52 & 4.02E-7 & 1.35E-5 & $9.12 \mathrm{E}-5$ \\
\hline SF-539 & 0.592 & 1.970 & 1.954 & 1.841 & 0.920 & 0.530 & 0.343 & 99 & 91 & 24 & -11 & -42 & 4.05E-7 & $4.93 \mathrm{E}-6$ & $>1.00 \mathrm{E}-4$ \\
\hline SNB-19 & 0.471 & 1.711 & 1.616 & 1.626 & 0.891 & 0.772 & 0.578 & 92 & 93 & 34 & 24 & 9 & $5.34 \mathrm{E}-7$ & $>1.00 \mathrm{E}-4$ & $>1.00 \mathrm{E}-4$ \\
\hline SNB-75 & 1.318 & 2.233 & 2.108 & 1.975 & 1.183 & 1.214 & 1.121 & 86 & 72 & -10 & -8 & -15 & $1.85 \mathrm{E}-7$ & $7.50 \mathrm{E}-7$ & $>1.00 \mathrm{E}-4$ \\
\hline U251 & 0.341 & 1.742 & 1.728 & 1.645 & 0.868 & 0.628 & 0.319 & 99 & 93 & 38 & 20 & -7 & $5.98 \mathrm{E}-7$ & $5.71 \mathrm{E}-5$ & $>1.00 \mathrm{E}-4$ \\
\hline Melanoma & & & & & & & & & & & & & & & \\
\hline LOX IM & 0.220 & 1.651 & 1.571 & 1.486 & 0.745 & 0.666 & 0.179 & 94 & 88 & 37 & 31 & -19 & $5.53 \mathrm{E}-7$ & $4.20 \mathrm{E}-5$ & $>1.00 \mathrm{E}-4$ \\
\hline MALME-3M & 0.598 & 1.037 & 1.000 & 0.984 & 0.815 & 0.858 & 0.404 & 92 & 88 & 49 & 59 & -32 & & $4.42 \mathrm{E}-5$ & $>1.00 \mathrm{E}-4$ \\
\hline & 0.642 & 2.410 & 2.288 & 2.183 & 1.107 & 0.876 & 0.73 & 93 & 87 & 26 & 13 & 5 & $4.08 \mathrm{E}-7$ & $>1.00 \mathrm{E}-4$ & $>1.00 \mathrm{E}-4$ \\
\hline MDA-MB-435 & 0.697 & 2.731 & 2.656 & 2.085 & 0.551 & 0.471 & 0.61 & 96 & 68 & -21 & -32 & -12 & $1.60 \mathrm{E}-7$ & $5.81 \mathrm{E}-7$ & $>1.00 \mathrm{E}-4$ \\
\hline SK-MEL & 0.948 & 1.777 & 1.817 & 1.735 & 1.419 & 1.456 & 0.69 & 105 & 95 & 57 & 61 & -27 & $1.34 \mathrm{E}-5$ & $4.95 \mathrm{E}-5$ & $>1.00 \mathrm{E}-4$ \\
\hline SK-M & 0.770 & 2.011 & 1.993 & 1.856 & 1.518 & 1.357 & 0.9 & 99 & 88 & 60 & 47 & 11 & $6.17 \mathrm{E}-6$ & $>1.00 \mathrm{E}-4$ & $>1.00 \mathrm{E}-4$ \\
\hline SK-M & 0.638 & 2.782 & 2.722 & 2.620 & 1.311 & 0.994 & 0.0 & 97 & 92 & 31 & 17 & -87 & $4.95 \mathrm{E}-7$ & & $4.41 \mathrm{E}-5$ \\
\hline UACC & 0.623 & 1.665 & 1.625 & 1.489 & 1.117 & 1.262 & 0.550 & 96 & 83 & 47 & 61 & -12 & & $6.90 \mathrm{E}-5$ & $>1.00 \mathrm{E}-4$ \\
\hline UACC-62 & 0.922 & 2.652 & 2.592 & 2.383 & 1.538 & 1.373 & 0.702 & 96 & 84 & 36 & 26 & -24 & $5.07 \mathrm{E}-7$ & $3.32 \mathrm{E}-5$ & $>1.00 \mathrm{E}-4$ \\
\hline Ovarian Cancer & & & & & & & & & & & & & & & \\
\hline IGROV1 & 0.425 & 1.800 & 1.745 & 1.760 & 0.925 & 0.738 & 0.474 & 96 & 97 & 36 & 23 & 4 & $5.96 \mathrm{E}-7$ & $>1.00 \mathrm{E}-4$ & $>1.00 \mathrm{E}-4$ \\
\hline & & 2.075 & 1.911 & 2.033 & 0.976 & 0.868 & $0.4 \varsigma$ & 87 & 97 & 16 & 8 & -36 & $3.78 \mathrm{E}-7$ & 9E-5 & $>1.00 \mathrm{E}-4$ \\
\hline & 0.7 & 1.737 & 1.749 & 1.676 & 1.293 & 1.035 & 0.8 & 101 & 94 & 57 & 32 & 10 & $1.91 \mathrm{E}-6$ & $>1.0$ & $>1.00 \mathrm{E}-4$ \\
\hline OVCAF & 0.4 & 1.386 & 1.322 & 1.296 & 1.109 & 0.706 & 0.532 & 93 & 90 & 69 & 24 & 4 & $2.63 \mathrm{E}$ & $>1.0$ & $>1.00 \mathrm{E}-4$ \\
\hline OVCAF & 0.4 & 2.208 & 2.140 & 2.060 & 1.397 & 0.810 & 0.452 & 96 & 92 & 55 & 22 & 2 & 1.39E-6 & $>1.0$ & $>1.00 \mathrm{E}-4$ \\
\hline NCI/ADR-RES & 0.420 & 1.509 & 1.484 & 1.471 & 0.786 & 0.509 & 0.400 & 98 & 97 & 34 & 8 & -5 & $5.49 \mathrm{E}-7$ & 4.23E-5 & $>1.00 \mathrm{E}-4$ \\
\hline SK-OV-3 & 0.860 & 1.789 & 1.813 & 1.787 & 1.421 & 1.269 & 0.871 & 103 & 100 & 60 & 44 & 1 & 4.30E-6 & $>1.00 \mathrm{E}-4$ & $>1.00 \mathrm{E}-4$ \\
\hline Renal C & & & & & & & & & & & & & & & \\
\hline & 0.738 & 2.807 & 2.688 & 2.568 & 1.416 & 1.357 & 0.782 & 94 & 88 & 33 & 30 & 2 & & & $1.00 \mathrm{E}-4$ \\
\hline & 1.682 & 2.254 & 2.167 & 2.122 & 1.599 & 1.515 & 1.33 & 85 & 77 & -5 & -10 & -21 & & & \\
\hline & 0.300 & 1.246 & 1.23 & 1.22 & 0.652 & 0.5 & 0.1 & 99 & 98 & 37 & 29 & -61 & & & $E-5$ \\
\hline & 0.78 & 2.4 & 2.325 & 2.06 & 1.362 & 1.3 & 0.7 & 95 & 79 & 36 & 36 & -11 & & & $>1.00$ \\
\hline & 1.01 & 1.78 & 1.731 & $1.6 \varsigma$ & 1.279 & 1.14 & 0.9 & 93 & 88 & 34 & 17 & -7 & & & $>1.00$ \\
\hline SN12C & 1.2 & 3.264 & 3.16 & 3.132 & 2.499 & 1.8 & 1.13 & 95 & 93 & 62 & 29 & -8 & & & $1.00 \mathrm{E}-4$ \\
\hline TK- & 0.872 & 1.714 & 1.653 & 1.552 & 1.45 & 1.249 & 0.8 & 93 & 81 & 69 & 45 & -3 & & & $\mathrm{E}-4$ \\
\hline บO-31 & 0.903 & 1.985 & 1.779 & 1.734 & 1.444 & 1.233 & 0.832 & 81 & 77 & 50 & 30 & -8 & $9.97 \mathrm{E}-7$ & $6.23 \mathrm{E}-5$ & $>1.00 \mathrm{E}-4$ \\
\hline & & & & & & & & & & & & & & & \\
\hline & 0.550 & 2.015 & 1.939 & 1.845 & 1.140 & 0.928 & 0.623 & 95 & 88 & 40 & 26 & 5 & $6.28 \mathrm{E}-7$ & $>1.00 \mathrm{E}-4$ & $>1.00 \mathrm{E}-4$ \\
\hline DU-145 & 0.429 & 1.664 & 1.771 & 1.697 & 1.279 & 0.723 & 0.515 & 109 & 103 & 69 & 24 & 7 & $2.62 \mathrm{E}-6$ & $>1.00 \mathrm{E}-4$ & $>1.00 \mathrm{E}-4$ \\
\hline Breast Can & & & & & & & & & & & & & & & \\
\hline MCF7 & 0.264 & 1.415 & 1.30 & 1.3 & 0.422 & $0.3 \varepsilon$ & 0.2 & 0 & 92 & 14 & 11 & -4 & & & \\
\hline MD & 0.704 & 1.51 & 1.533 & 1.45 & 1.198 & 0.838 & 0.4 & 102 & 93 & 61 & 16 & -32 & & & \\
\hline & 1.5 & 2.651 & 2.661 & 2.52 & 1.703 & 1.8 & 1.4 & 101 & 89 & 15 & 26 & -7 & & & \\
\hline & 1.118 & 2.393 & 2.333 & 2.235 & 1.645 & 1.435 & 0.984 & 95 & 88 & 41 & 25 & -12 & & 4.73 & $1.00 \mathrm{E}-4$ \\
\hline T-47D & 0.832 & 1.989 & 1.884 & 1.897 & 1.217 & 1.197 & 0.879 & 91 & 92 & 33 & 32 & 4 & $5.18 \mathrm{E}-7$ & $1.00 \mathrm{E}-4$ & $1.00 \mathrm{E}-4$ \\
\hline
\end{tabular}


In-Vitro Testing Results

\begin{tabular}{|l|l|l|l|}
\hline NSC : D - $816480 / 1$ & Experiment ID : 1909NS56 & Test Type : 08 & Units : Molar \\
\hline Report Date : October 25, 2019 & Test Date : September 09, 2019 & QNS : & MC : \\
\hline COMI : 2351710835 & Stain Reagent : SRB Dual-Pass Related & SSPL : OM6Y & \\
\hline
\end{tabular}

\begin{tabular}{|c|c|c|c|c|c|c|c|c|c|c|c|c|c|c|c|c|}
\hline & & & & & & & $g 10 \mathrm{Co}$ & tration & & & & & & & & \\
\hline & Time & & & Mean & Optical & I Densitic & & & & reent & rowth & & & & & \\
\hline Panel/Cell Line & Zero & Ctrl & -8.0 & -7.0 & -6.0 & -5.0 & -4.0 & -8.0 & -7.0 & -6.0 & -5.0 & -4.0 & GI50 & TGI & & LC50 \\
\hline Leukemia & & & & & & & & & & & & & & & & \\
\hline CCRF-CEM & 1.197 & 3.297 & 3.246 & 3.194 & 1.868 & 1.569 & 1.232 & 98 & 95 & 32 & 18 & 2 & $5.17 \mathrm{E}-7$ & $>1.00 \mathrm{E}-4$ & $>$ & $1.00 \mathrm{E}-4$ \\
\hline HL-60(TB) & 0.819 & 3.061 & 2.929 & 2.891 & 0.673 & 0.644 & 0.532 & 94 & 92 & -18 & -21 & -35 & $2.42 \mathrm{E}-7$ & $6.88 \mathrm{E}-7$ & $>$ & $1.00 \mathrm{E}-4$ \\
\hline $\mathrm{K}-562$ & 0.243 & 2.533 & 2.318 & 1.473 & 0.487 & 0.376 & 0.300 & 91 & 54 & 11 & 6 & 2 & $1.22 \mathrm{E}-7$ & $>1.00 \mathrm{E}-4$ & $>$ & $1.00 \mathrm{E}-4$ \\
\hline MOLT-4 & 1.240 & 3.413 & 3.425 & 3.429 & 2.293 & 1.707 & 1.067 & 101 & 101 & 48 & 21 & -14 & $9.34 \mathrm{E}-7$ & 4.04E-5 & $>$ & $1.00 \mathrm{E}-4$ \\
\hline RPMI-8226 & 1.165 & 2.859 & 2.801 & 2.831 & 1.847 & 1.453 & 0.887 & 97 & 98 & 40 & 17 & -24 & $6.79 \mathrm{E}-7$ & $2.61 \mathrm{E}-5$ & $>$ & $1.00 \mathrm{E}-4$ \\
\hline SR & 0.879 & 2.864 & 2.748 & 1.789 & 1.208 & 1.123 & 0.768 & 94 & 46 & 17 & 12 & -13 & $8.20 \mathrm{E}-8$ & 3.10E-5 & $>$ & $1.00 \mathrm{E}-4$ \\
\hline Non-Small Cell Lung & Cancer & & & & & & & & & & & & & & & \\
\hline A549/ATCC & 0.731 & 2.916 & 2.846 & 2.819 & 1.791 & 1.336 & 0.731 & 97 & 96 & 48 & 28 & & $9.29 \mathrm{E}-7$ & $9.94 \mathrm{E}-5$ & $>$ & $1.00 \mathrm{E}-4$ \\
\hline EKVX & 1.088 & 1.916 & 1.824 & 1.892 & 1.552 & 1.549 & 1.021 & 89 & 97 & 56 & 56 & -6 & 1.23E-5 & $7.95 \mathrm{E}-5$ & $>$ & $1.00 \mathrm{E}-4$ \\
\hline HOP-62 & 1.195 & 2.674 & 2.467 & 2.381 & 1.694 & 1.446 & 1.261 & 86 & 80 & 34 & 17 & 4 & 4.46E-7 & $>1.00 \mathrm{E}-4$ & $>$ & $1.00 \mathrm{E}-4$ \\
\hline HOP-92 & 1.576 & 2.255 & 2.052 & 2.073 & 1.788 & 1.500 & 1.063 & 70 & 73 & 31 & -5 & -33 & $3.57 \mathrm{E}-7$ & 7.35E-6 & $>$ & $1.00 \mathrm{E}-4$ \\
\hline $\mathrm{NCl}-\mathrm{H} 226$ & 1.998 & 3.209 & 3.125 & 3.162 & 2.999 & 2.930 & 2.228 & 93 & 96 & 83 & 77 & 19 & $2.92 \mathrm{E}-5$ & $>1.00 \mathrm{E}-4$ & $>$ & $1.00 \mathrm{E}-4$ \\
\hline $\mathrm{NCl}-\mathrm{H} 23$ & 0.735 & 2.631 & 2.551 & 2.540 & 1.701 & 1.269 & 0.758 & 96 & 95 & 51 & 28 & 1 & $1.10 \mathrm{E}-6$ & $>1.00 \mathrm{E}-4$ & $>$ & $1.00 \mathrm{E}-4$ \\
\hline $\mathrm{NCl}-\mathrm{H} 322 \mathrm{M}$ & 1.007 & 2.703 & 2.625 & 2.618 & 1.984 & 1.768 & 1.400 & 95 & 95 & 58 & 45 & 23 & $3.96 \mathrm{E}-6$ & $>1.00 \mathrm{E}-4$ & $>$ & $1.00 \mathrm{E}-4$ \\
\hline $\mathrm{NCl}-\mathrm{H} 460$ & 0.388 & 3.141 & 3.170 & 3.163 & 0.843 & 0.590 & 0.280 & 101 & 101 & 17 & 7 & -28 & $4.01 \mathrm{E}-7$ & 1.61E-5 & $>$ & $1.00 \mathrm{E}-4$ \\
\hline $\mathrm{NCl}-\mathrm{H} 522$ & 1.427 & 3.111 & 3.029 & 2.781 & 1.911 & 1.567 & 1.119 & 95 & 80 & 29 & 8 & -22 & $3.88 \mathrm{E}-7$ & $1.90 \mathrm{E}-5$ & $>$ & $1.00 \mathrm{E}-4$ \\
\hline Colon Cancer & & & & & & & & & & & & & & & & \\
\hline COLO 205 & 1.191 & 3.211 & 3.128 & 3.169 & 1.851 & 0.913 & 0.735 & 96 & 98 & 33 & -23 & -38 & $5.43 \mathrm{E}-7$ & $3.83 \mathrm{E}-6$ & $>$ & $1.00 \mathrm{E}-4$ \\
\hline HCC-2998 & 1.508 & 3.404 & 3.388 & 3.376 & 3.175 & 2.016 & 1.102 & 99 & 99 & 88 & 27 & -27 & 4.17E-6 & $3.15 \mathrm{E}-5$ & $>$ & $1.00 \mathrm{E}-4$ \\
\hline НСТ-116 & 0.262 & 2.551 & 2.477 & 2.498 & 0.635 & 0.588 & 0.250 & 97 & 98 & 16 & 14 & -5 & $3.85 \mathrm{E}-7$ & $5.71 \mathrm{E}-5$ & $>$ & $1.00 \mathrm{E}-4$ \\
\hline HCT-15 & 0.556 & 3.101 & 2.993 & 2.738 & 1.292 & 1.136 & 0.650 & 96 & 86 & 29 & 23 & 4 & 4.26E-7 & $>1.00 \mathrm{E}-4$ & $>$ & $1.00 \mathrm{E}-4$ \\
\hline HT29 & 0.345 & 2.469 & 2.388 & 2.354 & 0.655 & 0.488 & 0.267 & 96 & 95 & 15 & 7 & -23 & $3.61 \mathrm{E}-7$ & 1.69E-5 & $>$ & $1.00 \mathrm{E}-4$ \\
\hline KM12 & 0.607 & 2.996 & 2.880 & 2.435 & 1.146 & 1.073 & 0.434 & 95 & 76 & 23 & 19 & -29 & 3.10E-7 & $2.55 \mathrm{E}-5$ & $>$ & $1.00 \mathrm{E}-4$ \\
\hline SW-620 & 0.382 & 2.277 & 2.192 & 2.014 & 0.832 & 0.751 & 0.615 & 96 & 86 & 24 & 19 & 12 & $3.79 \mathrm{E}-7$ & $>1.00 \mathrm{E}-4$ & $>$ & $1.00 \mathrm{E}-4$ \\
\hline CNS Cancer & & & & & & & & & & & & & & & & \\
\hline SF-268 & 1.245 & 2.652 & 2.625 & 2.542 & 2.128 & 1.984 & 1.295 & 98 & 92 & 63 & 52 & 4 & $1.12 \mathrm{E}-5$ & $>1.00 \mathrm{E}-4$ & $>$ & $1.00 \mathrm{E}-4$ \\
\hline SF-295 & 1.036 & 3.205 & 3.122 & 3.042 & 1.446 & 1.155 & 0.552 & 96 & 92 & 19 & 5 & -47 & $3.78 \mathrm{E}-7$ & 1.27E-5 & $>$ & $1.00 \mathrm{E}-4$ \\
\hline SF-539 & 1.324 & 3.086 & 3.019 & 3.024 & 1.494 & 0.795 & 0.952 & 96 & 96 & 10 & -40 & -28 & 3.43E-7 & $1.56 \mathrm{E}-6$ & $>$ & $1.00 \mathrm{E}-4$ \\
\hline SNB-19 & 0.708 & 2.503 & 2.423 & 2.404 & 1.361 & 1.325 & 1.009 & 96 & 95 & 36 & 34 & 17 & $5.83 \mathrm{E}-7$ & $>1.00 \mathrm{E}-4$ & $>$ & $1.00 \mathrm{E}-4$ \\
\hline SNB-75 & 1.166 & 1.871 & 1.695 & 1.676 & 1.024 & 0.747 & 0.873 & 75 & 72 & -12 & -36 & -25 & $1.84 \mathrm{E}-7$ & $7.18 \mathrm{E}-7$ & $>$ & $1.00 \mathrm{E}-4$ \\
\hline U251 & 0.704 & 2.682 & 2.626 & 2.565 & 1.516 & 1.281 & 0.722 & 97 & 94 & 41 & 29 & 1 & $6.78 \mathrm{E}-7$ & $>1.00 \mathrm{E}-4$ & $>$ & $1.00 \mathrm{E}-4$ \\
\hline Melanoma & & & & & & & & & & & & & & & & \\
\hline LOX IMVI & 0.511 & 3.095 & 2.967 & 2.901 & 1.595 & 1.540 & 0.493 & 95 & 93 & 42 & 40 & -4 & $6.93 \mathrm{E}-7$ & $8.25 \mathrm{E}-5$ & $>$ & $1.00 \mathrm{E}-4$ \\
\hline MALME-3M & 1.107 & 2.633 & 2.547 & 2.268 & 1.577 & 1.753 & 0.833 & 94 & 76 & 31 & 42 & -25 & 3.76E-7 & 4.27E-5 & $>$ & $1.00 \mathrm{E}-4$ \\
\hline M14 & 1.523 & 3.342 & 3.320 & 3.324 & 2.163 & 2.479 & 1.393 & 99 & 99 & 35 & 53 & -9 & & $7.25 \mathrm{E}-5$ & $>$ & $1.00 \mathrm{E}-4$ \\
\hline MDA-MB-435 & 0.662 & 2.951 & 2.906 & 1.640 & 0.538 & 0.341 & 0.622 & 98 & 43 & -19 & -48 & -6 & $7.38 \mathrm{E}-8$ & 4.96E-7 & $>$ & $1.00 \mathrm{E}-4$ \\
\hline SK-MEL-2 & 1.526 & 3.125 & 3.101 & 3.064 & 2.320 & 2.152 & 1.617 & 98 & 96 & 50 & 39 & 6 & $9.83 \mathrm{E}-7$ & $>1.00 \mathrm{E}-4$ & $>$ & $1.00 \mathrm{E}-4$ \\
\hline SK-MEL-28 & 0.826 & 2.525 & 2.529 & 2.370 & 1.666 & 1.652 & 0.984 & 100 & 91 & 49 & 49 & 9 & $9.68 \mathrm{E}-7$ & $>1.00 \mathrm{E}-4$ & $>$ & $1.00 \mathrm{E}-4$ \\
\hline SK-MEL-5 & 0.918 & 3.249 & 3.217 & 3.144 & 1.395 & 1.269 & 0.042 & 99 & 95 & 20 & 15 & -95 & $4.04 \mathrm{E}-7$ & 1.37E-5 & & $3.88 \mathrm{E}-5$ \\
\hline UACC-257 & 2.076 & 3.168 & 3.092 & 3.071 & 2.964 & 2.998 & 1.545 & 93 & 91 & 81 & 84 & -26 & $2.05 \mathrm{E}-5$ & $5.85 \mathrm{E}-5$ & $>$ & $1.00 \mathrm{E}-4$ \\
\hline UACC-62 & 0.954 & 3.146 & 3.131 & 2.932 & 1.573 & 1.515 & 0.744 & 99 & 90 & 28 & 26 & -22 & 4.46E-7 & 3.45E-5 & $>$ & $1.00 \mathrm{E}-4$ \\
\hline Ovarian Cancer & & & & & & & & & & & & & & & & \\
\hline IGROV1 & 0.658 & 2.506 & 2.452 & 2.458 & 1.560 & 1.112 & 0.818 & 97 & 97 & 49 & 25 & 9 & $9.46 \mathrm{E}-7$ & $>1.00 \mathrm{E}-4$ & $>$ & $1.00 \mathrm{E}-4$ \\
\hline OVCAR-3 & 0.661 & 2.081 & 2.055 & 1.986 & 0.834 & 0.661 & 0.453 & 98 & 93 & 12 & & -31 & $3.41 \mathrm{E}-7$ & 1.00E-5 & $>$ & $1.00 \mathrm{E}-4$ \\
\hline OVCAR-4 & 0.833 & 2.024 & 1.998 & 1.985 & 1.516 & 1.278 & 0.931 & 98 & 97 & 57 & 37 & 8 & $2.33 \mathrm{E}-6$ & $>1.00 \mathrm{E}-4$ & $>$ & $1.00 \mathrm{E}-4$ \\
\hline OVCAR-5 & 0.895 & 1.778 & 1.679 & 1.705 & 1.380 & 1.232 & 1.167 & 89 & 92 & 55 & 38 & 31 & $1.97 \mathrm{E}-6$ & $>1.00 \mathrm{E}-4$ & $>$ & $1.00 \mathrm{E}-4$ \\
\hline OVCAR-8 & 0.631 & 2.821 & 2.714 & 2.789 & 1.837 & 1.258 & 0.771 & 95 & 99 & 55 & 29 & 6 & $1.55 \mathrm{E}-6$ & $>1.00 \mathrm{E}-4$ & $>$ & $1.00 \mathrm{E}-4$ \\
\hline NCI/ADR-RES & 0.550 & 2.016 & 1.992 & 1.743 & 0.844 & 0.569 & 0.549 & 98 & 81 & 20 & 1 & & $3.25 \mathrm{E}-7$ & $6.70 \mathrm{E}-5$ & $>$ & $1.00 \mathrm{E}-4$ \\
\hline SK-OV-3 & 1.816 & 2.541 & 2.549 & 2.591 & 2.245 & 2.068 & 1.493 & 101 & 107 & 59 & 35 & -18 & $2.37 \mathrm{E}-6$ & $4.58 \mathrm{E}-5$ & $>$ & $1.00 \mathrm{E}-4$ \\
\hline Renal Cancer & & & & & & & & & & & & & & & & \\
\hline $786-0$ & 1.013 & 3.035 & 2.965 & 3.036 & 1.869 & 1.671 & 0.960 & 97 & 100 & 42 & 33 & -5 & $7.36 \mathrm{E}-7$ & $7.25 \mathrm{E}-5$ & $>$ & $1.00 \mathrm{E}-4$ \\
\hline A498 & 2.142 & 2.935 & 2.899 & 2.833 & 1.885 & 1.592 & 1.758 & 95 & 87 & -12 & -26 & -18 & 2.37E-7 & $7.57 \mathrm{E}-7$ & $>$ & $1.00 \mathrm{E}-4$ \\
\hline $\mathrm{ACHN}$ & 0.399 & 1.814 & 1.813 & 1.827 & 0.934 & 0.861 & 0.447 & 100 & 101 & 38 & 33 & 3 & $6.41 \mathrm{E}-7$ & $>1.00 \mathrm{E}-4$ & $>$ & $1.00 \mathrm{E}-4$ \\
\hline CAKI-1 & 0.939 & 2.384 & 2.180 & 1.981 & 1.430 & 1.340 & 0.929 & 86 & 72 & 34 & 28 & -1 & $3.80 \mathrm{E}-7$ & $9.18 \mathrm{E}-5$ & $>$ & $1.00 \mathrm{E}-4$ \\
\hline RXF 393 & 1.406 & 2.057 & 1.964 & 1.962 & 1.420 & 1.032 & 1.189 & 86 & 85 & 2 & -27 & -15 & $2.66 \mathrm{E}-7$ & $1.18 \mathrm{E}-6$ & $>$ & $1.00 \mathrm{E}-4$ \\
\hline SN12C & 0.776 & 2.931 & 2.862 & 2.922 & 1.735 & 1.542 & 0.841 & 97 & 100 & 44 & 36 & 3 & $7.94 \mathrm{E}-7$ & $>1.00 \mathrm{E}-4$ & $>$ & $1.00 \mathrm{E}-4$ \\
\hline TK-10 & 0.802 & 1.717 & 1.644 & 1.636 & 1.472 & 1.264 & 0.827 & 92 & 91 & 73 & 50 & 3 & $1.02 \mathrm{E}-5$ & $>1.00 \mathrm{E}-4$ & $>$ & $1.00 \mathrm{E}-4$ \\
\hline UO-31 & 0.802 & 2.383 & 2.214 & 2.232 & 1.518 & 1.332 & 0.842 & 89 & 90 & 45 & 33 & 2 & $7.85 \mathrm{E}-7$ & $>1.00 \mathrm{E}-4$ & $>$ & $1.00 \mathrm{E}-4$ \\
\hline Prostate Cancer & & & & & & & & & & & & & & & & \\
\hline PC-3 & 0.612 & 2.347 & 2.203 & 2.164 & 1.303 & 0.988 & 0.693 & 92 & 89 & 40 & 22 & 5 & $6.23 \mathrm{E}-7$ & $>1.00 \mathrm{E}-4$ & $>$ & $1.00 \mathrm{E}-4$ \\
\hline DU-145 & 0.492 & 1.823 & 1.842 & 1.869 & 1.200 & 0.719 & 0.569 & 101 & 103 & 53 & 17 & 6 & $1.23 \mathrm{E}-6$ & $>1.00 \mathrm{E}-4$ & $>$ & $1.00 \mathrm{E}-4$ \\
\hline Breast Cancer & & & & & & & & & & & & & & & & \\
\hline MCF7 & 0.610 & 2.930 & 2.681 & 2.581 & 1.166 & 1.041 & 0.735 & 89 & 85 & 24 & 19 & 5 & $3.74 \mathrm{E}-7$ & $>1.00 \mathrm{E}-4$ & $>$ & $1.00 \mathrm{E}-4$ \\
\hline MDA-MB-231/ATCC & 0.843 & 2.056 & 1.996 & 1.971 & 1.475 & 1.152 & 0.810 & 95 & 93 & 52 & 25 & -4 & $1.20 \mathrm{E}-6$ & 7.33E-5 & $>$ & $1.00 \mathrm{E}-4$ \\
\hline HS 578T & 1.672 & 2.745 & 2.611 & 2.619 & 1.852 & 1.736 & 1.683 & 87 & 88 & 17 & 6 & 1 & 3.43E-7 & $>1.00 \mathrm{E}-4$ & $>$ & $1.00 \mathrm{E}-4$ \\
\hline BT-549 & 1.695 & 3.367 & 3.329 & 3.299 & 3.000 & 2.423 & 1.402 & 98 & 96 & 78 & 44 & -17 & $6.50 \mathrm{E}-6$ & $5.20 \mathrm{E}-5$ & $>$ & $1.00 \mathrm{E}-4$ \\
\hline T-47D & 1.165 & 2.430 & 2.214 & 2.328 & 1.496 & 1.301 & 1.028 & 83 & 92 & 26 & 11 & -12 & 4.34E-7 & 3.00E-5 & $>$ & $1.00 \mathrm{E}-4$ \\
\hline MDA-MB-468 & 0.973 & 2.356 & 2.209 & 2.135 & 1.010 & 0.929 & 0.705 & 89 & 84 & 3 & -5 & -28 & $2.62 \mathrm{E}-7$ & $2.34 \mathrm{E}-6$ & $>$ & $1.00 \mathrm{E}-4$ \\
\hline
\end{tabular}


In-Vitro Testing Results

\begin{tabular}{|l|l|l|l|}
\hline NSC : D - 823371 / 1 & Experiment ID : 2008NS40 & Test Type : 08 & Units : Molar \\
\hline Report Date : March 24, 2021 & Test Date : August 24, 2020 & QNS : & MC: \\
\hline COMI : KLB2-16 & Stain Reagent : SRB Dual-Pass Related & SSPL : 1C1X & \\
\hline
\end{tabular}

Log10 Concentration

\begin{tabular}{|c|c|c|c|c|c|c|c|c|c|c|c|c|c|c|c|}
\hline \multirow[b]{2}{*}{ Panel/Cell Line } & \multicolumn{3}{|l|}{ Time } & \multicolumn{5}{|c|}{ Mean Optical Densities } & \multicolumn{4}{|c|}{ Percent Growth } & \multirow[b]{2}{*}{ GI50 } & \multirow[b]{2}{*}{ TGI } & \multirow[b]{2}{*}{ LC50 } \\
\hline & Zero & Ctrl & -8.0 & -7.0 & -6.0 & -5.0 & -4.0 & -8.0 & -7.0 & -6.0 & -5.0 & -4.0 & & & \\
\hline $\begin{array}{l}\text { Leukemia } \\
\text { CCRF-CEM } \\
\text { HL-60(TB) } \\
\text { K-562 } \\
\text { MOLT-4 } \\
\text { RPMI-8226 } \\
\text { SR }\end{array}$ & $\begin{array}{l}0.354 \\
0.732 \\
0.198 \\
0.633 \\
0.988 \\
0.289\end{array}$ & $\begin{array}{l}1.502 \\
2.541 \\
1.711 \\
2.681 \\
2.351 \\
1.589\end{array}$ & $\begin{array}{l}1.540 \\
2.585 \\
1.691 \\
2.739 \\
2.350 \\
1.426\end{array}$ & $\begin{array}{l}1.523 \\
2.535 \\
1.385 \\
2.788 \\
2.377 \\
1.332\end{array}$ & $\begin{array}{l}0.567 \\
0.722 \\
0.382 \\
1.332 \\
1.323 \\
0.494\end{array}$ & $\begin{array}{l}0.501 \\
0.697 \\
0.315 \\
0.894 \\
1.134 \\
0.425\end{array}$ & $\begin{array}{l}0.185 \\
0.362 \\
0.189 \\
0.396 \\
0.862 \\
0.206\end{array}$ & $\begin{array}{r}103 \\
102 \\
99 \\
103 \\
100 \\
87\end{array}$ & $\begin{array}{r}102 \\
100 \\
78 \\
105 \\
102 \\
80\end{array}$ & $\begin{array}{r}19 \\
-1 \\
12 \\
34 \\
25 \\
16\end{array}$ & $\begin{array}{r}13 \\
-5 \\
8 \\
13 \\
11 \\
10\end{array}$ & $\begin{array}{l}-48 \\
-51 \\
-5 \\
-37 \\
-13 \\
-29\end{array}$ & $\begin{array}{l}4.19 \mathrm{E}-7 \\
3.10 \mathrm{E}-7 \\
2.68 \mathrm{E}-7 \\
5.98 \mathrm{E}-7 \\
4.69 \mathrm{E}-7 \\
2.94 \mathrm{E}-7\end{array}$ & $\begin{array}{l}1.63 \mathrm{E}-5 \\
9.68 \mathrm{E}-7 \\
4.26 \mathrm{E}-5 \\
1.79 \mathrm{E}-5 \\
2.85 \mathrm{E}-5 \\
1.85 \mathrm{E}-5\end{array}$ & $\begin{array}{r}>1.00 \mathrm{E}-4 \\
\quad 9.70 \mathrm{E}-5 \\
>1.00 \mathrm{E}-4 \\
>1.00 \mathrm{E}-4 \\
>1.00 \mathrm{E}-4 \\
>1.00 \mathrm{E}-4\end{array}$ \\
\hline $\begin{array}{l}\text { Non-Small Cell Lung } \\
\text { A549/ATCC } \\
\text { EKVX } \\
\text { HOP-62 } \\
\text { HOP-92 } \\
\text { NCl-H226 } \\
\text { NCl-H23 } \\
\text { NCl-H322M } \\
\text { NCl-H460 } \\
\text { NCl-H522 }\end{array}$ & $\begin{array}{l}\text { Cancer } \\
0.481 \\
0.645 \\
1.021 \\
1.294 \\
1.250 \\
0.659 \\
0.932 \\
0.543 \\
0.960\end{array}$ & $\begin{array}{l}2.407 \\
1.988 \\
2.479 \\
2.052 \\
2.899 \\
1.848 \\
2.279 \\
3.320 \\
2.365 \\
\end{array}$ & $\begin{array}{l}2.322 \\
1.858 \\
2.343 \\
1.884 \\
2.862 \\
1.822 \\
2.191 \\
3.323 \\
2.215\end{array}$ & $\begin{array}{l}2.355 \\
1.857 \\
2.335 \\
1.891 \\
2.917 \\
1.854 \\
2.226 \\
3.332 \\
1.923\end{array}$ & $\begin{array}{l}1.321 \\
1.292 \\
1.563 \\
1.588 \\
1.917 \\
1.362 \\
1.721 \\
1.195 \\
0.982\end{array}$ & $\begin{array}{l}0.758 \\
0.877 \\
1.292 \\
1.180 \\
1.453 \\
0.889 \\
1.495 \\
0.788 \\
0.924\end{array}$ & $\begin{array}{l}0.117 \\
0.094 \\
0.127 \\
0.116 \\
0.346 \\
0.101 \\
0.014 \\
0.099 \\
0.175\end{array}$ & $\begin{array}{r}96 \\
90 \\
91 \\
78 \\
98 \\
98 \\
93 \\
100 \\
89\end{array}$ & $\begin{array}{r}97 \\
90 \\
90 \\
79 \\
101 \\
101 \\
96 \\
100 \\
69\end{array}$ & $\begin{array}{r}44 \\
48 \\
37 \\
39 \\
40 \\
59 \\
59 \\
23 \\
2\end{array}$ & $\begin{array}{r}14 \\
17 \\
19 \\
-9 \\
12 \\
19 \\
42 \\
9 \\
-4\end{array}$ & $\begin{array}{l}-76 \\
-86 \\
-88 \\
-91 \\
-72 \\
-85 \\
-99 \\
-82 \\
-82\end{array}$ & $\begin{array}{l}7.60 \mathrm{E}-7 \\
9.05 \mathrm{E}-7 \\
5.73 \mathrm{E}-7 \\
5.23 \mathrm{E}-7 \\
6.96 \mathrm{E}-7 \\
1.69 \mathrm{E}-6 \\
3.23 \mathrm{E}-6 \\
4.52 \mathrm{E}-7 \\
1.89 \mathrm{E}-7\end{array}$ & $\begin{array}{l}1.44 \mathrm{E}-5 \\
1.47 \mathrm{E}-5 \\
1.50 \mathrm{E}-5 \\
6.53 \mathrm{E}-6 \\
1.40 \mathrm{E}-5 \\
1.53 \mathrm{E}-5 \\
1.99 \mathrm{E}-5 \\
1.25 \mathrm{E}-5 \\
1.94 \mathrm{E}-6\end{array}$ & $\begin{array}{l}5.19 \mathrm{E}-5 \\
4.51 \mathrm{E}-5 \\
4.42 \mathrm{E}-5 \\
3.17 \mathrm{E}-5 \\
5.45 \mathrm{E}-5 \\
4.64 \mathrm{E}-5 \\
4.51 \mathrm{E}-5 \\
4.46 \mathrm{E}-5 \\
3.91 \mathrm{E}-5\end{array}$ \\
\hline $\begin{array}{l}\text { Colon Cancer } \\
\text { COLO } 205 \\
\text { HCC-2998 } \\
\text { HCT-116 } \\
\text { HCT-15 } \\
\text { HT29 } \\
\text { KM12 } \\
\text { SW-620 }\end{array}$ & $\begin{array}{l}0.420 \\
0.664 \\
0.188 \\
0.271 \\
0.337 \\
0.632 \\
0.325\end{array}$ & $\begin{array}{l}1.450 \\
1.970 \\
2.195 \\
1.580 \\
1.955 \\
2.870 \\
2.009\end{array}$ & $\begin{array}{l}1.494 \\
1.909 \\
2.027 \\
1.465 \\
1.973 \\
2.797 \\
1.896\end{array}$ & $\begin{array}{l}1.462 \\
1.919 \\
1.964 \\
1.436 \\
1.897 \\
2.712 \\
1.909\end{array}$ & $\begin{array}{l}0.769 \\
1.542 \\
0.597 \\
0.570 \\
0.402 \\
1.101 \\
0.600\end{array}$ & $\begin{array}{l}0.389 \\
0.837 \\
0.391 \\
0.417 \\
0.321 \\
0.897 \\
0.654\end{array}$ & $\begin{array}{l}0.080 \\
0.087 \\
0.018 \\
0.017 \\
0.050 \\
0.050 \\
0.039\end{array}$ & $\begin{array}{r}104 \\
95 \\
92 \\
91 \\
101 \\
97 \\
93\end{array}$ & $\begin{array}{c}101 \\
96 \\
88 \\
89 \\
96 \\
93 \\
94\end{array}$ & $\begin{array}{r}34 \\
67 \\
20 \\
23 \\
4 \\
21 \\
16\end{array}$ & $\begin{array}{l}-7 \\
13 \\
10 \\
11 \\
-5 \\
12 \\
20\end{array}$ & $\begin{array}{l}-81 \\
-87 \\
-91 \\
-94 \\
-85 \\
-92 \\
-88\end{array}$ & $\begin{array}{l}5.77 \mathrm{E}-7 \\
2.08 \mathrm{E}-6 \\
3.67 \mathrm{E}-7 \\
3.88 \mathrm{E}-7 \\
3.18 \mathrm{E}-7 \\
3.95 \mathrm{E}-7 \\
3.69 \mathrm{E}-7\end{array}$ & $\begin{array}{l}6.63 \mathrm{E}-6 \\
1.36 \mathrm{E}-5 \\
1.26 \mathrm{E}-5 \\
1.28 \mathrm{E}-5 \\
2.86 \mathrm{E}-6 \\
1.30 \mathrm{E}-5 \\
1.52 \mathrm{E}-5\end{array}$ & $\begin{array}{l}3.80 \mathrm{E}-5 \\
4.28 \mathrm{E}-5 \\
3.95 \mathrm{E}-5 \\
3.82 \mathrm{E}-5 \\
3.64 \mathrm{E}-5 \\
3.94 \mathrm{E}-5 \\
4.42 \mathrm{E}-5\end{array}$ \\
\hline $\begin{array}{l}\text { CNS Cancer } \\
\text { SF-268 } \\
\text { SF-295 } \\
\text { SF-539 } \\
\text { SNB-19 } \\
\text { SNB-75 } \\
\text { U251 }\end{array}$ & $\begin{array}{l}0.965 \\
0.871 \\
0.592 \\
0.471 \\
1.318 \\
0.341\end{array}$ & $\begin{array}{l}2.455 \\
2.947 \\
1.936 \\
1.717 \\
2.206 \\
1.691\end{array}$ & $\begin{array}{l}2.374 \\
2.645 \\
1.877 \\
1.659 \\
1.967 \\
1.593\end{array}$ & $\begin{array}{l}2.355 \\
2.810 \\
1.883 \\
1.670 \\
1.974 \\
1.620\end{array}$ & $\begin{array}{l}1.694 \\
1.338 \\
0.836 \\
0.827 \\
1.130 \\
0.671\end{array}$ & $\begin{array}{l}1.351 \\
0.930 \\
0.417 \\
0.755 \\
0.993 \\
0.511\end{array}$ & $\begin{array}{l}0.264 \\
0.055 \\
0.024 \\
0.028 \\
0.068 \\
0.024\end{array}$ & $\begin{array}{l}95 \\
85 \\
96 \\
95 \\
73 \\
93\end{array}$ & $\begin{array}{l}93 \\
93 \\
96 \\
96 \\
74 \\
95\end{array}$ & $\begin{array}{r}49 \\
22 \\
18 \\
29 \\
-14 \\
24\end{array}$ & $\begin{array}{r}26 \\
3 \\
-30 \\
23 \\
-25 \\
13\end{array}$ & $\begin{array}{l}-73 \\
-94 \\
-96 \\
-94 \\
-95 \\
-93\end{array}$ & $\begin{array}{l}9.46 \mathrm{E}-7 \\
4.09 \mathrm{E}-7 \\
3.90 \mathrm{E}-7 \\
4.82 \mathrm{E}-7 \\
1.86 \mathrm{E}-7 \\
4.33 \mathrm{E}-7\end{array}$ & $\begin{array}{l}1.83 \mathrm{E}-5 \\
1.07 \mathrm{E}-5 \\
2.40 \mathrm{E}-6 \\
1.57 \mathrm{E}-5 \\
6.88 \mathrm{E}-7 \\
1.32 \mathrm{E}-5\end{array}$ & $\begin{array}{l}5.89 \mathrm{E}-5 \\
3.52 \mathrm{E}-5 \\
2.03 \mathrm{E}-5 \\
4.19 \mathrm{E}-5 \\
2.30 \mathrm{E}-5 \\
3.91 \mathrm{E}-5\end{array}$ \\
\hline $\begin{array}{l}\text { Melanoma } \\
\text { LOX IMVI } \\
\text { MALME-3M } \\
\text { M14 } \\
\text { MDA-MB-435 } \\
\text { SK-MEL-2 } \\
\text { SK-MEL-28 } \\
\text { SK-MEL-5 } \\
\text { UACC-257 } \\
\text { UACC-62 }\end{array}$ & $\begin{array}{l}0.220 \\
0.598 \\
0.642 \\
0.697 \\
0.948 \\
0.770 \\
0.638 \\
0.623 \\
0.922\end{array}$ & $\begin{array}{l}1.534 \\
1.056 \\
2.331 \\
2.665 \\
1.825 \\
1.990 \\
2.636 \\
1.632 \\
2.737\end{array}$ & $\begin{array}{l}1.483 \\
1.038 \\
2.113 \\
2.577 \\
1.824 \\
2.060 \\
2.550 \\
1.620 \\
2.626\end{array}$ & $\begin{array}{l}1.479 \\
1.022 \\
2.033 \\
1.877 \\
1.802 \\
1.917 \\
2.553 \\
1.533 \\
2.524\end{array}$ & $\begin{array}{l}0.665 \\
0.809 \\
1.016 \\
0.473 \\
1.344 \\
1.436 \\
1.231 \\
1.116 \\
1.497\end{array}$ & $\begin{array}{l}0.605 \\
0.812 \\
0.819 \\
0.487 \\
1.285 \\
1.196 \\
0.807 \\
0.923 \\
1.200\end{array}$ & $\begin{array}{l}0.048 \\
0.122 \\
0.134 \\
0.013 \\
0.155 \\
0.017 \\
0.005 \\
0.052 \\
0.023\end{array}$ & $\begin{array}{r}96 \\
96 \\
87 \\
96 \\
100 \\
106 \\
96 \\
99 \\
94\end{array}$ & $\begin{array}{l}96 \\
93 \\
82 \\
60 \\
97 \\
94 \\
96 \\
90 \\
88\end{array}$ & $\begin{array}{r}34 \\
46 \\
22 \\
-32 \\
45 \\
55 \\
30 \\
49 \\
32\end{array}$ & $\begin{array}{r}29 \\
47 \\
10 \\
-30 \\
38 \\
35 \\
8 \\
30 \\
15\end{array}$ & $\begin{array}{l}-78 \\
-80 \\
-79 \\
-98 \\
-84 \\
-98 \\
-99 \\
-92 \\
-98\end{array}$ & $\begin{array}{l}5.49 \mathrm{E}-7 \\
8.21 \mathrm{E}-7 \\
3.44 \mathrm{E}-7 \\
1.28 \mathrm{E}-7 \\
8.08 \mathrm{E}-7 \\
1.71 \mathrm{E}-6 \\
4.93 \mathrm{E}-7 \\
9.37 \mathrm{E}-7 \\
4.74 \mathrm{E}-7\end{array}$ & $\begin{array}{l}1.87 \mathrm{E}-5 \\
2.34 \mathrm{E}-5 \\
1.31 \mathrm{E}-5 \\
4.48 \mathrm{E}-7 \\
2.06 \mathrm{E}-5 \\
1.83 \mathrm{E}-5 \\
1.20 \mathrm{E}-5 \\
1.76 \mathrm{E}-5 \\
1.37 \mathrm{E}-5\end{array}$ & $\begin{array}{l}5.45 \mathrm{E}-5 \\
5.83 \mathrm{E}-5 \\
4.73 \mathrm{E}-5 \\
1.95 \mathrm{E}-5 \\
5.30 \mathrm{E}-5 \\
4.36 \mathrm{E}-5 \\
3.49 \mathrm{E}-5 \\
4.54 \mathrm{E}-5 \\
3.79 \mathrm{E}-5\end{array}$ \\
\hline $\begin{array}{l}\text { Ovarian Cancer } \\
\text { IGROV1 } \\
\text { OVCAR-3 } \\
\text { OVCAR-4 } \\
\text { OVCAR-5 } \\
\text { OVCAR-8 } \\
\text { NCI/ADR-RES } \\
\text { SK-OV-3 }\end{array}$ & $\begin{array}{l}0.425 \\
0.770 \\
0.704 \\
0.494 \\
0.419 \\
0.420 \\
0.860\end{array}$ & $\begin{array}{l}1.803 \\
2.012 \\
1.710 \\
1.386 \\
2.140 \\
1.512 \\
1.806\end{array}$ & $\begin{array}{l}1.778 \\
2.022 \\
1.693 \\
1.344 \\
2.048 \\
1.516 \\
1.813\end{array}$ & $\begin{array}{l}1.694 \\
2.004 \\
1.699 \\
1.341 \\
2.144 \\
1.468 \\
1.808\end{array}$ & $\begin{array}{l}0.879 \\
0.766 \\
1.280 \\
1.015 \\
1.225 \\
0.726 \\
1.274\end{array}$ & $\begin{array}{l}0.679 \\
0.688 \\
0.897 \\
0.635 \\
0.754 \\
0.533 \\
1.122\end{array}$ & $\begin{array}{l}0.085 \\
0.009 \\
0.215 \\
0.011 \\
0.149 \\
0.245 \\
0.044\end{array}$ & $\begin{array}{r}98 \\
101 \\
98 \\
95 \\
95 \\
100 \\
101\end{array}$ & $\begin{array}{r}92 \\
99 \\
99 \\
95 \\
100 \\
96 \\
100\end{array}$ & $\begin{array}{r}33 \\
57 \\
58 \\
47 \\
28 \\
44\end{array}$ & $\begin{array}{r}18 \\
-11 \\
19 \\
16 \\
19 \\
10 \\
28\end{array}$ & $\begin{array}{l}-80 \\
-99 \\
-69 \\
-98 \\
-65 \\
-42 \\
-95\end{array}$ & $\begin{array}{l}5.15 \mathrm{E}-7 \\
3.12 \mathrm{E}-7 \\
1.55 \mathrm{E}-6 \\
1.57 \mathrm{E}-6 \\
8.71 \mathrm{E}-7 \\
4.74 \mathrm{E}-7 \\
7.73 \mathrm{E}-7\end{array}$ & $\begin{array}{l}1.54 \mathrm{E}-5 \\
9.88 \mathrm{E}-7 \\
1.64 \mathrm{E}-5 \\
1.38 \mathrm{E}-5 \\
1.70 \mathrm{E}-5 \\
1.58 \mathrm{E}-5 \\
1.68 \mathrm{E}-5\end{array}$ & $\begin{array}{r}4.95 \mathrm{E}-5 \\
2.79 \mathrm{E}-5 \\
6.03 \mathrm{E}-5 \\
3.79 \mathrm{E}-5 \\
6.71 \mathrm{E}-5 \\
>\quad 1.00 \mathrm{E}-4 \\
4.30 \mathrm{E}-5\end{array}$ \\
\hline $\begin{array}{l}\text { Renal Cancer } \\
786-0 \\
\text { A498 } \\
\text { ACHN } \\
\text { CAKI-1 } \\
\text { RXF 393 } \\
\text { SN12C } \\
\text { TK-10 } \\
\text { UO-31 }\end{array}$ & $\begin{array}{l}0.738 \\
1.682 \\
0.300 \\
0.784 \\
1.018 \\
1.234 \\
0.872 \\
0.903\end{array}$ & $\begin{array}{l}2.734 \\
2.243 \\
1.223 \\
2.278 \\
1.813 \\
3.276 \\
1.725 \\
1.967\end{array}$ & $\begin{array}{l}2.663 \\
2.150 \\
1.241 \\
2.029 \\
1.759 \\
3.191 \\
1.583 \\
1.797\end{array}$ & $\begin{array}{l}2.599 \\
2.197 \\
1.235 \\
1.960 \\
1.734 \\
3.213 \\
1.616 \\
1.847\end{array}$ & $\begin{array}{l}1.339 \\
1.667 \\
0.629 \\
1.207 \\
1.285 \\
2.281 \\
1.343 \\
1.469\end{array}$ & $\begin{array}{l}1.109 \\
1.422 \\
0.527 \\
1.144 \\
1.040 \\
1.677 \\
0.983 \\
1.112\end{array}$ & $\begin{array}{l}0.242 \\
0.060 \\
0.003 \\
0.015 \\
0.309 \\
0.034 \\
0.041 \\
0.149\end{array}$ & $\begin{array}{r}96 \\
83 \\
102 \\
83 \\
93 \\
96 \\
83 \\
84\end{array}$ & $\begin{array}{r}93 \\
92 \\
101 \\
79 \\
90 \\
97 \\
87 \\
89\end{array}$ & $\begin{array}{l}30 \\
. \\
36 \\
28 \\
34 \\
51 \\
55 \\
53\end{array}$ & $\begin{array}{r}19 \\
-15 \\
25 \\
24 \\
3 \\
22 \\
13 \\
20\end{array}$ & $\begin{array}{l}-67 \\
-96 \\
-99 \\
-98 \\
-70 \\
-97 \\
-95 \\
-83\end{array}$ & $\begin{array}{l}4.84 \mathrm{E}-7 \\
2.82 \mathrm{E}-7 \\
6.04 \mathrm{E}-7 \\
3.71 \mathrm{E}-7 \\
5.11 \mathrm{E}-7 \\
1.10 \mathrm{E}-6 \\
1.33 \mathrm{E}-6 \\
1.24 \mathrm{E}-6\end{array}$ & $\begin{array}{l}1.65 \mathrm{E}-5 \\
9.77 \mathrm{E}-7 \\
1.58 \mathrm{E}-5 \\
1.57 \mathrm{E}-5 \\
1.09 \mathrm{E}-5 \\
1.52 \mathrm{E}-5 \\
1.32 \mathrm{E}-5 \\
1.55 \mathrm{E}-5\end{array}$ & $\begin{array}{l}6.30 \mathrm{E}-5 \\
2.67 \mathrm{E}-5 \\
4.00 \mathrm{E}-5 \\
4.04 \mathrm{E}-5 \\
5.35 \mathrm{E}-5 \\
4.01 \mathrm{E}-5 \\
3.82 \mathrm{E}-5 \\
4.73 \mathrm{E}-5\end{array}$ \\
\hline $\begin{array}{l}\text { Prostate Cancer } \\
\text { PC-3 } \\
\text { DU-145 }\end{array}$ & $\begin{array}{l}0.550 \\
0.429\end{array}$ & $\begin{array}{l}1.970 \\
1.662\end{array}$ & $\begin{array}{l}1.846 \\
1.666\end{array}$ & $\begin{array}{l}1.864 \\
1.650\end{array}$ & $\begin{array}{l}1.113 \\
1.150\end{array}$ & $\begin{array}{l}0.752 \\
0.652\end{array}$ & $\begin{array}{l}0.068 \\
0.010\end{array}$ & $\begin{array}{r}91 \\
100\end{array}$ & $\begin{array}{l}93 \\
99\end{array}$ & $\begin{array}{l}40 \\
58\end{array}$ & $\begin{array}{l}14 \\
18\end{array}$ & $\begin{array}{l}-88 \\
-98\end{array}$ & $\begin{array}{l}6.37 \mathrm{E}-7 \\
1.62 \mathrm{E}-6\end{array}$ & $\begin{array}{l}1.38 \mathrm{E}-5 \\
1.43 \mathrm{E}-5\end{array}$ & $\begin{array}{l}4.27 \mathrm{E}-5 \\
3.87 \mathrm{E}-5\end{array}$ \\
\hline $\begin{array}{l}\text { Breast Cancer } \\
\text { MCF7 } \\
\text { MDA-MB-231/ATCC } \\
\text { HS 578T } \\
\text { BT-549 } \\
\text { T-47D }\end{array}$ & $\begin{array}{l}0.264 \\
0.704 \\
1.536 \\
1.118 \\
0.832\end{array}$ & $\begin{array}{l}1.405 \\
1.587 \\
2.611 \\
2.356 \\
1.952\end{array}$ & $\begin{array}{l}1.284 \\
1.546 \\
2.442 \\
2.284 \\
1.790\end{array}$ & $\begin{array}{l}1.324 \\
1.551 \\
2.575 \\
2.282 \\
1.828\end{array}$ & $\begin{array}{l}0.386 \\
1.124 \\
1.692 \\
1.556 \\
1.125\end{array}$ & $\begin{array}{l}0.344 \\
0.731 \\
1.478 \\
1.338 \\
1.008\end{array}$ & $\begin{array}{l}0.054 \\
0.200 \\
0.750 \\
0.088 \\
0.338\end{array}$ & $\begin{array}{l}89 \\
95 \\
84 \\
94 \\
86\end{array}$ & $\begin{array}{l}93 \\
96 \\
97 \\
94 \\
89\end{array}$ & $\begin{array}{l}11 \\
48 \\
15 \\
35 \\
26\end{array}$ & $\begin{array}{r}7 \\
3 \\
-4 \\
18 \\
16\end{array}$ & $\begin{array}{l}-80 \\
-72 \\
-51 \\
-92 \\
-59\end{array}$ & $\begin{array}{l}3.33 \mathrm{E}-7 \\
8.89 \mathrm{E}-7 \\
3.70 \mathrm{E}-7 \\
5.63 \mathrm{E}-7 \\
4.17 \mathrm{E}-7\end{array}$ & $\begin{array}{l}1.21 \mathrm{E}-5 \\
1.10 \mathrm{E}-5 \\
6.20 \mathrm{E}-6 \\
1.45 \mathrm{E}-5 \\
1.62 \mathrm{E}-5\end{array}$ & $\begin{array}{l}4.56 \mathrm{E}-5 \\
5.14 \mathrm{E}-5 \\
9.43 \mathrm{E}-5 \\
4.14 \mathrm{E}-5 \\
7.49 \mathrm{E}-5\end{array}$ \\
\hline
\end{tabular}


In-Vitro Testing Results

\begin{tabular}{|l|l|l|l|}
\hline NSC : D - 824737 / 1 & Experiment ID : 2009NS50 & Test Type : 08 & Units : Molar \\
\hline Report Date : March 24, 2021 & Test Date : September 28, 2020 & QNS : & MC: \\
\hline COMI : KLB2-17 & Stain Reagent : SRB Dual-Pass Related & SSPL : 1C1X & \\
\hline
\end{tabular}

Log10 Concentration

\begin{tabular}{|c|c|c|c|c|c|c|c|c|c|c|c|c|c|c|c|c|}
\hline \multirow[b]{2}{*}{ Panel/Cell Line } & \multirow{2}{*}{$\begin{array}{l}\text { Time } \\
\text { Zero }\end{array}$} & \multirow[b]{2}{*}{ Ctrl } & \multicolumn{5}{|c|}{ Mean Optical Densities } & \multicolumn{5}{|c|}{ Percent Growth } & \multirow[b]{2}{*}{ GI50 } & \multirow[b]{2}{*}{ TGI } & \multirow{2}{*}{\multicolumn{2}{|c|}{ LC50 }} \\
\hline & & & -8.0 & -7.0 & -6.0 & -5.0 & -4.0 & -8.0 & -7.0 & -6.0 & -5.0 & -4.0 & & & & \\
\hline $\begin{array}{l}\text { Leukemia } \\
\text { CCRF-CEM } \\
\text { HL-60(TB) } \\
\text { K-562 } \\
\text { MOLT-4 } \\
\text { SR }\end{array}$ & $\begin{array}{l}0.425 \\
0.585 \\
0.154 \\
0.589 \\
0.269\end{array}$ & $\begin{array}{l}2.106 \\
2.834 \\
1.709 \\
2.884 \\
0.762\end{array}$ & $\begin{array}{l}2.077 \\
2.746 \\
1.713 \\
2.919 \\
0.758\end{array}$ & $\begin{array}{l}2.069 \\
2.767 \\
1.465 \\
2.908 \\
0.685\end{array}$ & $\begin{array}{l}1.059 \\
0.972 \\
0.363 \\
1.475 \\
0.408\end{array}$ & $\begin{array}{l}0.677 \\
0.626 \\
0.315 \\
1.159 \\
0.390\end{array}$ & $\begin{array}{l}0.461 \\
0.480 \\
0.151 \\
0.548 \\
0.206\end{array}$ & $\begin{array}{r}98 \\
96 \\
100 \\
102 \\
99\end{array}$ & $\begin{array}{r}98 \\
97 \\
84 \\
101 \\
84\end{array}$ & $\begin{array}{l}38 \\
17 \\
13 \\
39 \\
28\end{array}$ & $\begin{array}{r}15 \\
2 \\
10 \\
25 \\
24\end{array}$ & $\begin{array}{r}2 \\
-18 \\
-2 \\
-7 \\
-23\end{array}$ & $\begin{array}{l}6.24 \mathrm{E}-7 \\
3.88 \mathrm{E}-7 \\
3.05 \mathrm{E}-7 \\
6.57 \mathrm{E}-7 \\
4.09 \mathrm{E}-7\end{array}$ & $\begin{array}{r}\text { 1.00E-4 } \\
1.23 \mathrm{E}-5 \\
6.60 \mathrm{E}-5 \\
6.01 \mathrm{E}-5 \\
3.24 \mathrm{E}-5\end{array}$ & $\begin{array}{l}> \\
> \\
> \\
> \\
>\end{array}$ & $\begin{array}{l}1.00 \mathrm{E}-4 \\
1.00 \mathrm{E}-4 \\
1.00 \mathrm{E}-4 \\
1.00 \mathrm{E}-4 \\
1.00 \mathrm{E}-4\end{array}$ \\
\hline $\begin{array}{l}\text { Non-Small Cell Lung } \\
\text { A549/ATCC } \\
\text { EKVX } \\
\text { HOP-62 } \\
\text { HOP-92 } \\
\mathrm{NCl}-\mathrm{H} 226 \\
\mathrm{NCl}-\mathrm{H} 23 \\
\mathrm{NCl}-\mathrm{H} 322 \mathrm{M} \\
\mathrm{NCl}-\mathrm{H} 460 \\
\mathrm{NCl}-\mathrm{H} 522\end{array}$ & $\begin{array}{l}\text { Cancer } \\
0.452 \\
0.611 \\
1.085 \\
1.137 \\
0.796 \\
0.699 \\
0.919 \\
0.319 \\
1.273\end{array}$ & $\begin{array}{l}2.508 \\
2.041 \\
2.911 \\
1.775 \\
1.571 \\
2.262 \\
2.397 \\
3.137 \\
2.826\end{array}$ & $\begin{array}{l}2.444 \\
1.941 \\
2.849 \\
1.695 \\
1.523 \\
2.145 \\
2.390 \\
3.136 \\
2.652\end{array}$ & $\begin{array}{l}2.510 \\
1.998 \\
2.740 \\
1.663 \\
1.546 \\
2.199 \\
2.330 \\
3.187 \\
2.595\end{array}$ & $\begin{array}{l}1.543 \\
1.537 \\
1.887 \\
1.519 \\
1.265 \\
1.643 \\
1.995 \\
0.941 \\
1.823\end{array}$ & $\begin{array}{l}1.068 \\
1.079 \\
1.514 \\
1.258 \\
0.973 \\
1.151 \\
1.603 \\
0.699 \\
1.501\end{array}$ & $\begin{array}{l}0.377 \\
0.481 \\
0.140 \\
0.176 \\
0.207 \\
0.247 \\
0.491 \\
0.170 \\
0.566\end{array}$ & $\begin{array}{r}97 \\
93 \\
97 \\
87 \\
94 \\
93 \\
100 \\
100 \\
89\end{array}$ & $\begin{array}{r}100 \\
97 \\
91 \\
83 \\
97 \\
96 \\
95 \\
102 \\
85\end{array}$ & $\begin{array}{l}53 \\
65 \\
44 \\
60 \\
60 \\
60 \\
73 \\
22 \\
35\end{array}$ & $\begin{array}{l}30 \\
33 \\
23 \\
19 \\
23 \\
29 \\
46 \\
13 \\
15\end{array}$ & $\begin{array}{l}-17 \\
-21 \\
-87 \\
-85 \\
-74 \\
-65 \\
-47 \\
-47 \\
-56\end{array}$ & $\begin{array}{l}1.35 \mathrm{E}-6 \\
2.88 \mathrm{E}-6 \\
7.40 \mathrm{E}-7 \\
1.74 \mathrm{E}-6 \\
1.90 \mathrm{E}-6 \\
2.14 \mathrm{E}-6 \\
7.24 \mathrm{E}-6 \\
4.46 \mathrm{E}-7 \\
5.09 \mathrm{E}-7\end{array}$ & $\begin{array}{l}4.40 \mathrm{E}-5 \\
4.03 \mathrm{E}-5 \\
1.63 \mathrm{E}-5 \\
1.52 \mathrm{E}-5 \\
1.72 \mathrm{E}-5 \\
2.04 \mathrm{E}-5 \\
3.15 \mathrm{E}-5 \\
1.67 \mathrm{E}-5 \\
1.62 \mathrm{E}-5\end{array}$ & $>$ & $\begin{array}{l}1.00 \mathrm{E}-4 \\
1.00 \mathrm{E}-4 \\
4.62 \mathrm{E}-5 \\
4.63 \mathrm{E}-5 \\
5.65 \mathrm{E}-5 \\
6.97 \mathrm{E}-5 \\
1.00 \mathrm{E}-4 \\
1.00 \mathrm{E}-4 \\
8.34 \mathrm{E}-5\end{array}$ \\
\hline $\begin{array}{l}\text { CNS Cancer } \\
\text { SF-268 } \\
\text { SF-295 } \\
\text { SF-539 } \\
\text { SNB-19 } \\
\text { SNB-75 } \\
\text { U251 }\end{array}$ & $\begin{array}{l}1.025 \\
0.622 \\
0.527 \\
0.646 \\
1.147 \\
0.453\end{array}$ & $\begin{array}{l}2.629 \\
2.430 \\
2.086 \\
2.295 \\
2.023 \\
2.219\end{array}$ & $\begin{array}{l}2.506 \\
2.280 \\
2.032 \\
2.248 \\
1.756 \\
2.159\end{array}$ & $\begin{array}{l}2.466 \\
2.266 \\
2.059 \\
2.270 \\
1.751 \\
2.168\end{array}$ & $\begin{array}{l}2.013 \\
1.024 \\
1.157 \\
1.494 \\
1.230 \\
1.229\end{array}$ & $\begin{array}{l}1.652 \\
0.771 \\
0.640 \\
1.297 \\
0.884 \\
0.979\end{array}$ & $\begin{array}{l}0.426 \\
0.027 \\
0.043 \\
0.441 \\
0.357 \\
0.132\end{array}$ & $\begin{array}{l}92 \\
92 \\
97 \\
97 \\
70 \\
97\end{array}$ & $\begin{array}{l}90 \\
91 \\
98 \\
98 \\
69 \\
97\end{array}$ & $\begin{array}{r}62 \\
22 \\
40 \\
51 \\
9 \\
44\end{array}$ & $\begin{array}{r}39 \\
8 \\
7 \\
39 \\
-23 \\
30\end{array}$ & $\begin{array}{l}-58 \\
-96 \\
-92 \\
-32 \\
-69 \\
-71\end{array}$ & $\begin{array}{l}3.28 \mathrm{E}-6 \\
3.94 \mathrm{E}-7 \\
6.83 \mathrm{E}-7 \\
1.31 \mathrm{E}-6 \\
2.08 \mathrm{E}-7 \\
7.69 \mathrm{E}-7\end{array}$ & $\begin{array}{l}2.52 \mathrm{E}-5 \\
1.20 \mathrm{E}-5 \\
1.18 \mathrm{E}-5 \\
3.58 \mathrm{E}-5 \\
1.96 \mathrm{E}-6 \\
1.98 \mathrm{E}-5\end{array}$ & $>$ & $\begin{array}{l}8.19 \mathrm{E}-5 \\
3.63 \mathrm{E}-5 \\
3.78 \mathrm{E}-5 \\
1.00 \mathrm{E}-4 \\
3.88 \mathrm{E}-5 \\
6.20 \mathrm{E}-5\end{array}$ \\
\hline $\begin{array}{l}\text { Melanoma } \\
\text { LOX IMVI } \\
\text { MALME-3M } \\
\text { M14 } \\
\text { MDA-MB-435 } \\
\text { SK-MEL-2 } \\
\text { SK-MEL-28 } \\
\text { SK-MEL-5 } \\
\text { UACC-257 } \\
\text { UACC-62 }\end{array}$ & $\begin{array}{l}0.281 \\
0.751 \\
0.536 \\
0.836 \\
0.913 \\
0.545 \\
1.093 \\
0.814 \\
0.745\end{array}$ & $\begin{array}{l}2.236 \\
1.715 \\
2.358 \\
2.347 \\
2.051 \\
1.824 \\
3.267 \\
2.291 \\
2.629\end{array}$ & $\begin{array}{l}2.160 \\
1.669 \\
2.307 \\
2.145 \\
2.032 \\
1.854 \\
3.273 \\
2.150 \\
2.463\end{array}$ & $\begin{array}{l}2.130 \\
1.713 \\
2.275 \\
2.018 \\
2.037 \\
1.829 \\
3.294 \\
2.211 \\
2.373\end{array}$ & $\begin{array}{l}1.028 \\
1.258 \\
1.205 \\
1.282 \\
1.378 \\
1.322 \\
2.476 \\
1.731 \\
1.352\end{array}$ & $\begin{array}{l}0.922 \\
1.244 \\
1.120 \\
1.159 \\
1.249 \\
1.198 \\
1.502 \\
1.520 \\
1.150\end{array}$ & $\begin{array}{r}0.026 \\
0.374 \\
0.263 \\
0.215 \\
0.309 \\
0.054 \\
-0.006 \\
0.394 \\
0.038\end{array}$ & $\begin{array}{r}96 \\
95 \\
97 \\
87 \\
98 \\
102 \\
100 \\
90 \\
91\end{array}$ & $\begin{array}{r}95 \\
100 \\
95 \\
78 \\
99 \\
100 \\
101 \\
95 \\
86\end{array}$ & $\begin{array}{l}38 \\
53 \\
37 \\
30 \\
41 \\
61 \\
64 \\
62 \\
32\end{array}$ & $\begin{array}{c}33 \\
51 \\
32 \\
21 \\
29 \\
51 \\
19 \\
48 \\
21\end{array}$ & $\begin{array}{r}-91 \\
-50 \\
-51 \\
-74 \\
-66 \\
-90 \\
-100 \\
-52 \\
-95\end{array}$ & $\begin{array}{l}6.17 \mathrm{E}-7 \\
1.03 \mathrm{E}-5 \\
5.93 \mathrm{E}-7 \\
3.79 \mathrm{E}-7 \\
6.95 \mathrm{E}-7 \\
1.02 \mathrm{E}-5 \\
2.01 \mathrm{E}-6 \\
6.98 \mathrm{E}-6 \\
4.70 \mathrm{E}-7\end{array}$ & $\begin{array}{l}1.84 \mathrm{E}-5 \\
3.19 \mathrm{E}-5 \\
2.43 \mathrm{E}-5 \\
1.67 \mathrm{E}-5 \\
2.03 \mathrm{E}-5 \\
2.30 \mathrm{E}-5 \\
1.44 \mathrm{E}-5 \\
3.02 \mathrm{E}-5 \\
1.53 \mathrm{E}-5\end{array}$ & & $\begin{array}{l}4.67 \mathrm{E}-5 \\
9.95 \mathrm{E}-5 \\
9.72 \mathrm{E}-5 \\
5.57 \mathrm{E}-5 \\
6.77 \mathrm{E}-5 \\
5.19 \mathrm{E}-5 \\
3.79 \mathrm{E}-5 \\
9.62 \mathrm{E}-5 \\
4.11 \mathrm{E}-5\end{array}$ \\
\hline $\begin{array}{l}\text { Ovarian Cancer } \\
\text { IGROV1 } \\
\text { OVCAR-3 } \\
\text { OVCAR-4 } \\
\text { OVCAR-5 } \\
\text { OVCAR-8 } \\
\text { NCI/ADR-RES } \\
\text { SK-OV-3 }\end{array}$ & $\begin{array}{l}0.635 \\
0.560 \\
0.924 \\
0.469 \\
0.492 \\
0.402 \\
1.128\end{array}$ & $\begin{array}{l}2.395 \\
1.872 \\
1.862 \\
1.549 \\
2.521 \\
1.577 \\
2.137\end{array}$ & $\begin{array}{l}2.372 \\
1.883 \\
1.825 \\
1.531 \\
2.480 \\
1.562 \\
2.211\end{array}$ & $\begin{array}{l}2.354 \\
1.851 \\
1.812 \\
1.499 \\
2.429 \\
1.582 \\
2.203\end{array}$ & $\begin{array}{l}1.332 \\
0.828 \\
1.607 \\
1.144 \\
1.662 \\
0.807 \\
1.794\end{array}$ & $\begin{array}{l}1.014 \\
0.666 \\
1.147 \\
0.786 \\
1.129 \\
0.583 \\
1.574\end{array}$ & $\begin{array}{l}0.363 \\
0.094 \\
0.163 \\
0.084 \\
0.348 \\
0.195 \\
0.368\end{array}$ & $\begin{array}{r}99 \\
101 \\
96 \\
98 \\
98 \\
99 \\
107\end{array}$ & $\begin{array}{r}98 \\
98 \\
95 \\
95 \\
95 \\
100 \\
106\end{array}$ & $\begin{array}{l}40 \\
20 \\
73 \\
63 \\
58 \\
34 \\
66\end{array}$ & $\begin{array}{r}22 \\
8 \\
24 \\
29 \\
31 \\
15 \\
44\end{array}$ & $\begin{array}{l}-43 \\
-83 \\
-82 \\
-82 \\
-29 \\
-52 \\
-67\end{array}$ & $\begin{array}{l}6.62 \mathrm{E}-7 \\
4.17 \mathrm{E}-7 \\
2.92 \mathrm{E}-6 \\
2.39 \mathrm{E}-6 \\
1.95 \mathrm{E}-6 \\
5.81 \mathrm{E}-7 \\
5.41 \mathrm{E}-6\end{array}$ & $\begin{array}{l}2.16 \mathrm{E}-5 \\
1.23 \mathrm{E}-5 \\
1.68 \mathrm{E}-5 \\
1.83 \mathrm{E}-5 \\
3.29 \mathrm{E}-5 \\
1.70 \mathrm{E}-5 \\
2.49 \mathrm{E}-5\end{array}$ & $>$ & $\begin{array}{l}1.00 \mathrm{E}-4 \\
4.33 \mathrm{E}-5 \\
4.96 \mathrm{E}-5 \\
5.15 \mathrm{E}-5 \\
1.00 \mathrm{E}-4 \\
9.46 \mathrm{E}-5 \\
6.99 \mathrm{E}-5\end{array}$ \\
\hline $\begin{array}{l}\text { Renal Cancer } \\
786-0 \\
\text { A498 } \\
\text { ACHN } \\
\text { CAKI-1 } \\
\text { RXF 393 } \\
\text { SN12C } \\
\text { TK-10 } \\
\text { UO-31 }\end{array}$ & $\begin{array}{l}0.679 \\
1.155 \\
0.368 \\
0.692 \\
0.969 \\
0.541 \\
0.980 \\
0.612\end{array}$ & $\begin{array}{l}2.611 \\
2.030 \\
1.773 \\
2.259 \\
1.615 \\
2.231 \\
1.966 \\
2.029\end{array}$ & $\begin{array}{l}2.515 \\
2.036 \\
1.716 \\
2.187 \\
1.589 \\
2.146 \\
1.854 \\
1.882\end{array}$ & $\begin{array}{l}2.571 \\
2.007 \\
1.732 \\
1.809 \\
1.598 \\
2.198 \\
1.820 \\
1.853\end{array}$ & $\begin{array}{l}1.909 \\
1.376 \\
1.048 \\
0.898 \\
1.266 \\
1.431 \\
1.766 \\
1.390\end{array}$ & $\begin{array}{l}1.463 \\
1.142 \\
0.835 \\
0.861 \\
0.992 \\
1.220 \\
1.352 \\
1.083\end{array}$ & $\begin{array}{l}0.446 \\
0.108 \\
0.275 \\
0.256 \\
0.139 \\
0.078 \\
0.476 \\
0.072\end{array}$ & $\begin{array}{r}95 \\
101 \\
96 \\
95 \\
96 \\
95 \\
89 \\
90\end{array}$ & $\begin{array}{l}98 \\
97 \\
97 \\
71 \\
97 \\
98 \\
85 \\
88\end{array}$ & $\begin{array}{l}64 \\
25 \\
48 \\
13 \\
46 \\
53 \\
80 \\
55\end{array}$ & $\begin{array}{r}41 \\
-1 \\
33 \\
11 \\
3 \\
40 \\
38 \\
33\end{array}$ & $\begin{array}{l}-34 \\
-91 \\
-25 \\
-63 \\
-86 \\
-86 \\
-51 \\
-88\end{array}$ & $\begin{array}{l}3.90 \mathrm{E}-6 \\
4.53 \mathrm{E}-7 \\
9.25 \mathrm{E}-7 \\
2.32 \mathrm{E}-7 \\
8.35 \mathrm{E}-7 \\
1.62 \mathrm{E}-6 \\
5.10 \mathrm{E}-6 \\
1.68 \mathrm{E}-6\end{array}$ & $\begin{array}{l}3.48 \mathrm{E}-5 \\
9.03 \mathrm{E}-6 \\
3.70 \mathrm{E}-5 \\
1.40 \mathrm{E}-5 \\
1.09 \mathrm{E}-5 \\
2.09 \mathrm{E}-5 \\
2.65 \mathrm{E}-5 \\
1.88 \mathrm{E}-5\end{array}$ & $\begin{array}{l}> \\
>\end{array}$ & $\begin{array}{l}1.00 \mathrm{E}-4 \\
3.51 \mathrm{E}-5 \\
1.00 \mathrm{E}-4 \\
6.65 \mathrm{E}-5 \\
3.98 \mathrm{E}-5 \\
5.21 \mathrm{E}-5 \\
9.63 \mathrm{E}-5 \\
4.84 \mathrm{E}-5\end{array}$ \\
\hline $\begin{array}{l}\text { Prostate Cancer } \\
\text { PC-3 } \\
\text { DU-145 }\end{array}$ & $\begin{array}{l}0.454 \\
0.408\end{array}$ & $\begin{array}{l}2.033 \\
1.825\end{array}$ & $\begin{array}{l}1.947 \\
1.898\end{array}$ & $\begin{array}{l}1.776 \\
1.880\end{array}$ & $\begin{array}{l}1.130 \\
1.426\end{array}$ & $\begin{array}{l}0.768 \\
0.704\end{array}$ & $\begin{array}{l}0.176 \\
0.310\end{array}$ & $\begin{array}{r}95 \\
105\end{array}$ & $\begin{array}{r}84 \\
104\end{array}$ & $\begin{array}{l}43 \\
72\end{array}$ & $\begin{array}{l}20 \\
21\end{array}$ & $\begin{array}{l}-61 \\
-24\end{array}$ & $\begin{array}{l}6.68 \mathrm{E}-7 \\
2.68 \mathrm{E}-6\end{array}$ & $\begin{array}{l}1.76 \mathrm{E}-5 \\
2.91 \mathrm{E}-5\end{array}$ & $>$ & $\begin{array}{l}\text { 7.27E-5 } \\
1.00 \mathrm{E}-4\end{array}$ \\
\hline $\begin{array}{l}\text { Breast Cancer } \\
\text { MCF7 } \\
\text { MDA-MB-231/ATCC } \\
\text { HS 578T } \\
\text { BT-549 } \\
\text { T-47D } \\
\text { MDA-MB-468 }\end{array}$ & $\begin{array}{l}0.332 \\
0.429 \\
1.505 \\
1.085 \\
1.153 \\
0.815\end{array}$ & $\begin{array}{l}2.206 \\
1.184 \\
2.385 \\
2.222 \\
2.548 \\
1.804\end{array}$ & $\begin{array}{l}2.032 \\
1.202 \\
2.295 \\
2.138 \\
2.371 \\
1.758\end{array}$ & $\begin{array}{l}2.016 \\
1.230 \\
2.330 \\
2.214 \\
2.446 \\
1.752\end{array}$ & $\begin{array}{l}0.771 \\
0.876 \\
1.868 \\
1.669 \\
1.613 \\
1.031\end{array}$ & $\begin{array}{l}0.655 \\
0.698 \\
1.593 \\
1.511 \\
1.338 \\
0.857\end{array}$ & $\begin{array}{l}0.309 \\
0.137 \\
0.974 \\
0.350 \\
0.860 \\
0.452\end{array}$ & $\begin{array}{r}91 \\
102 \\
90 \\
93 \\
87 \\
95\end{array}$ & $\begin{array}{r}90 \\
106 \\
94 \\
99 \\
93 \\
95\end{array}$ & $\begin{array}{l}23 \\
59 \\
41 \\
51 \\
33 \\
22\end{array}$ & $\begin{array}{r}17 \\
36 \\
10 \\
37 \\
13 \\
4\end{array}$ & $\begin{array}{l}-7 \\
-68 \\
-35 \\
-68 \\
-25 \\
-45\end{array}$ & $\begin{array}{l}3.98 \mathrm{E}-7 \\
2.45 \mathrm{E}-6 \\
6.79 \mathrm{E}-7 \\
1.25 \mathrm{E}-6 \\
5.19 \mathrm{E}-7 \\
4.11 \mathrm{E}-7\end{array}$ & $\begin{array}{l}5.16 \mathrm{E}-5 \\
2.20 \mathrm{E}-5 \\
1.66 \mathrm{E}-5 \\
2.27 \mathrm{E}-5 \\
2.20 \mathrm{E}-5 \\
1.22 \mathrm{E}-5\end{array}$ & $\begin{array}{l}> \\
> \\
> \\
>\end{array}$ & $\begin{array}{l}1.00 \mathrm{E}-4 \\
6.68 \mathrm{E}-5 \\
1.00 \mathrm{E}-4 \\
6.78 \mathrm{E}-5 \\
1.00 \mathrm{E}-4 \\
1.00 \mathrm{E}-4\end{array}$ \\
\hline
\end{tabular}


In-Vitro Testing Results

\begin{tabular}{|l|l|l|l|}
\hline NSC : D - 823372 / 1 & Experiment ID : 2008NS40 & Test Type : 08 & Units : Molar \\
\hline Report Date : March 24, 2021 & Test Date : August 24, 2020 & QNS : & MC: \\
\hline COMI : KLB2-18 & Stain Reagent : SRB Dual-Pass Related & SSPL : 1C1X & \\
\hline
\end{tabular}

Log10 Concentration

\begin{tabular}{|c|c|c|c|c|c|c|c|c|c|c|c|c|c|c|c|}
\hline \multirow[b]{2}{*}{ Panel/Cell Line } & \multirow{2}{*}{$\begin{array}{l}\text { Time } \\
\text { Zero }\end{array}$} & \multirow[b]{2}{*}{ Ctrl } & \multicolumn{5}{|c|}{ Mean Optical Densities } & \multicolumn{5}{|c|}{ Percent Growth } & \multirow[b]{2}{*}{ GI50 } & \multirow[b]{2}{*}{ TGI } & \\
\hline & & & -8.0 & -7.0 & -6.0 & -5.0 & -4.0 & -8.0 & -7.0 & -6.0 & -5.0 & -4.0 & & & LC50 \\
\hline $\begin{array}{l}\text { Leukemia } \\
\text { CCRF-CEM }\end{array}$ & 0.354 & 1502 & 1529 & 1.167 & 0.541 & 0.494 & 0.209 & 102 & 71 & 16 & 12 & -41 & & & \\
\hline HL-60(TB) & 0.732 & 2.541 & 2.485 & 2.074 & 0.715 & 0.606 & 0.363 & 97 & 74 & $\begin{array}{l}10 \\
-2\end{array}$ & -17 & -50 & $\begin{array}{l}2.41 \mathrm{IE}-1 \\
2.07 \mathrm{E}-7\end{array}$ & $\begin{array}{l}1.09 \mathrm{E}-5 \\
9.31 \mathrm{E}-7\end{array}$ & $9.72 \mathrm{E}-5$ \\
\hline K-562 & 0.198 & 1.711 & 1.815 & 0.643 & 0.400 & 0.366 & 0.223 & 107 & 29 & 13 & 11 & 2 & $5.42 \mathrm{E}-8$ & $>1.00 \mathrm{E}-4$ & $1.00 \mathrm{E}-4$ \\
\hline MOLT-4 & 0.633 & 2.681 & 2.794 & 2.410 & 1.300 & 0.862 & 0.522 & 106 & 87 & 33 & 11 & -18 & $4.77 \mathrm{E}-7$ & $2.45 \mathrm{E}-5$ & 1.00E-4 \\
\hline RPMI-8226 & 0.988 & 2.351 & 2.348 & 2.030 & 1.196 & 1.160 & 0.826 & 100 & 76 & 15 & 13 & -16 & $2.70 \mathrm{E}-7$ & $2.72 \mathrm{E}-5$ & $1.00 \mathrm{E}-4$ \\
\hline SR & 0.289 & 1.589 & 1.437 & 0.672 & 0.513 & 0.448 & 0.252 & 88 & 29 & 17 & 12 & -13 & $4.48 \mathrm{E}-8$ & 3.06E-5 & $>1.00 \mathrm{E}-4$ \\
\hline Non-Small Cell Lung & Cancer & & & & & & & & & & & & & & \\
\hline A549/ATCC & 0.481 & 2.407 & 2.308 & 1.665 & 1.257 & 0.742 & 0.177 & 95 & 61 & 40 & 14 & -63 & $3.48 \mathrm{E}-7$ & $1.50 \mathrm{E}-5$ & $6.73 \mathrm{E}-5$ \\
\hline EKVX & 0.645 & 1.988 & 1.895 & 1.704 & 1.131 & 0.946 & 0.178 & 93 & 79 & 36 & 22 & -72 & 4.74E-7 & $1.72 \mathrm{E}-5$ & $5.79 \mathrm{E}-5$ \\
\hline HOP-62 & 1.021 & 2.479 & 2.331 & 1.975 & 1.562 & 1.484 & 0.243 & 90 & 65 & 37 & 32 & -76 & $3.51 \mathrm{E}-7$ & $1.97 \mathrm{E}-5$ & $5.72 E-5$ \\
\hline HOP-92 & 1.294 & 2.052 & 1.945 & 1.749 & 1.625 & 1.383 & 0.148 & 86 & 60 & 44 & 12 & -89 & 4.07E-7 & 1.31E-5 & 4.13E-5 \\
\hline $\mathrm{NCl}-\mathrm{H} 226$ & 1.250 & 2.899 & 2.899 & 2.727 & 1.918 & 1.544 & 0.315 & 100 & 90 & 41 & 18 & -75 & 6.40E-7 & $1.56 \mathrm{E}-5$ & $5.40 \mathrm{E}-5$ \\
\hline $\mathrm{NCl}-\mathrm{H} 23$ & 0.659 & 1.848 & 1.744 & 1.604 & 1.164 & 0.881 & 0.136 & 91 & 79 & 42 & 19 & -79 & $6.26 \mathrm{E}-7$ & 1.55E-5 & $5.01 \mathrm{E}-5$ \\
\hline $\mathrm{NCl}-\mathrm{H} 322 \mathrm{M}$ & 0.932 & 2.279 & 2.214 & 2149 & 1.486 & 1566 & 0.039 & 95 & 90 & 41 & 47 & -96 & $6.60 E-7$ & $213 \mathrm{E}-5$ & $4.78 \mathrm{E}-5$ \\
\hline $\mathrm{NCl}-\mathrm{H} 460$ & 0.543 & 3320 & 3.257 & 2971 & 1049 & 0.741 & 0.13 & 98 & 87 & 18 & 7 & -75 & $3.47 \mathrm{E}-7$ & $122 \mathrm{E}-5$ & $\begin{array}{l}\begin{array}{l}4.1 \% 0-5 \\
4.99 E-5\end{array} \\
4\end{array}$ \\
\hline $\mathrm{NCl}-\mathrm{H} 522$ & 0.960 & 2.365 & 2.247 & 1.367 & $\begin{array}{l}1.127 \\
\text { 1. }\end{array}$ & 0.916 & 0.260 & $\begin{array}{l}90 \\
92\end{array}$ & $\begin{array}{l}01 \\
29\end{array}$ & $\begin{array}{l}10 \\
12\end{array}$ & -5 & $\begin{array}{l}-10 \\
-73\end{array}$ & $\begin{array}{l}3.41 \mathrm{E}-8 \\
4.61\end{array}$ & $5.23 \mathrm{E}-6$ & $4.61 \mathrm{E}-5$ \\
\hline Colon Cancer & & & & & & & & & & & & & & & \\
\hline COLO 205 & 0.420 & 1.450 & 1.391 & 1.195 & 0.602 & 0.339 & 0.134 & 94 & 75 & 18 & -19 & -68 & $2.74 \mathrm{E}-7$ & $2.99 \mathrm{E}-6$ & $4.25 \mathrm{E}-5$ \\
\hline HCC-2998 & 0.664 & 1.970 & 1.888 & 1.709 & 1.398 & 0.766 & 0.126 & 94 & 80 & 56 & 8 & -81 & $1.34 \mathrm{E}-6$ & $1.22 \mathrm{E}-5$ & $4.47 \mathrm{E}-5$ \\
\hline HCT-116 & 0.188 & 2.195 & 2.113 & 1.325 & 0.837 & 0.449 & 0.056 & 96 & 57 & 32 & 13 & -70 & $1.88 \mathrm{E}-7$ & $1.43 \mathrm{E}-5$ & $5.68 \mathrm{E}-5$ \\
\hline HCT-15 & 0.271 & 1.580 & 1.479 & 0.940 & 0.613 & 0.392 & 0.058 & 92 & 51 & 26 & 9 & -79 & 1.10E-7 & $1.27 \mathrm{E}-5$ & $4.73 \mathrm{E}-5$ \\
\hline HT29 & 0.337 & 1.955 & 1.927 & 1.270 & 0.381 & 0.357 & 0.106 & 98 & 58 & 3 & 1 & -69 & $1.38 \mathrm{E}-7$ & $1.04 \mathrm{E}-5$ & $5.42 \mathrm{E}-5$ \\
\hline KM12 & 0.632 & 2.870 & 2.898 & 1.907 & 1.401 & 0.900 & 0.151 & 101 & 57 & 34 & 12 & -76 & $2.03 \mathrm{E}-7$ & 1.37E-5 & $5.05 \mathrm{E}-5$ \\
\hline SW-620 & 0.325 & 2.009 & 1.905 & 1.180 & 0.600 & 0.633 & 0.071 & 94 & 51 & 16 & 18 & -78 & $1.05 \mathrm{E}-7$ & $1.55 \mathrm{E}-5$ & $5.09 \mathrm{E}-5$ \\
\hline CNS Cancer & & & & & & & & & & & & & & & \\
\hline SF-268 & 0.965 & 2.455 & 2.374 & 2.163 & 1.845 & 1.354 & 0.362 & 95 & 80 & 59 & 26 & -62 & $1.88 \mathrm{E}-6$ & $1.97 \mathrm{E}-5$ & $7.23 \mathrm{E}-5$ \\
\hline SF-295 & 0.871 & 2.947 & 2.772 & 1.920 & 1.413 & 0.973 & 0.068 & 92 & 51 & 26 & 5 & -92 & $1.05 \mathrm{E}-7$ & $1.12 \mathrm{E}-5$ & 3.68E-5 \\
\hline SF-539 & 0.592 & 1.936 & 1.914 & 1.573 & 0.698 & 0.443 & 0.045 & 98 & 73 & 8 & -25 & -92 & $2.25 \mathrm{E}-7$ & $1.73 \mathrm{E}-6$ & $2.33 \mathrm{E}-5$ \\
\hline SNB-19 & 0.471 & 1.717 & 1.636 & 1.312 & 0.854 & 0.722 & 0.055 & 93 & 67 & 31 & 20 & -88 & $2.99 \mathrm{E}-7$ & $1.53 \mathrm{E}-5$ & $4.43 \mathrm{E}-5$ \\
\hline SNB-75 & 1.318 & 2.206 & 2.037 & 1.746 & 1.044 & 1.171 & 0.088 & 81 & 48 & -21 & -11 & -93 & & $4.99 \mathrm{E}-7$ & $2.97 \mathrm{E}-5$ \\
\hline U251 & 0.341 & 1.691 & 1.613 & 1.322 & 0.689 & 0.532 & 0.046 & 94 & 73 & 26 & 14 & -87 & $3.04 \mathrm{E}-7$ & $1.38 \mathrm{E}-5$ & $4.33 \mathrm{E}-5$ \\
\hline Melanoma & & & & & & & & & & & & & & & \\
\hline LOX IMVI & 0.220 & 1.534 & 1.458 & 1.062 & 0.646 & 0.399 & 0.045 & 94 & 64 & 32 & 14 & -80 & $2.77 \mathrm{E}-7$ & $1.40 \mathrm{E}-5$ & $4.82 \mathrm{E}-5$ \\
\hline MALME-3M & 0.598 & 1.056 & 1.029 & 0.875 & 0.828 & 0.881 & 0.094 & 94 & 61 & 50 & 62 & -84 & $1.21 \mathrm{E}-5$ & $2.65 \mathrm{E}-5$ & $5.83 \mathrm{E}-5$ \\
\hline M14 & 0.642 & 2.331 & 2.156 & 1.598 & 1.068 & 1.010 & 0.205 & 90 & 57 & 25 & 22 & -68 & $1.62 \mathrm{E}-7$ & $1.75 \mathrm{E}-5$ & $6.29 \mathrm{E}-5$ \\
\hline MDA-MB-435 & 0.697 & 2.665 & 2.538 & 0.766 & 0.330 & 0.547 & 0.023 & 94 & 4 & -53 & -22 & -97 & $3.04 \mathrm{E}-8$ & $1.15 \mathrm{E}-7$ & \\
\hline SK-MEL-2 & 0.948 & 1.825 & 1.770 & 1.588 & 1.328 & 1.359 & 0.298 & 94 & 73 & 43 & 47 & -69 & $5.93 \mathrm{E}-7$ & $2.55 \mathrm{E}-5$ & $6.90 \mathrm{E}-5$ \\
\hline SK-MEL-28 & 0.770 & 1.990 & 2.017 & 1.640 & 1.531 & 1.274 & 0.054 & 102 & 71 & 62 & 41 & -93 & $3.87 \mathrm{E}-6$ & $2.03 \mathrm{E}-5$ & $4.79 \mathrm{E}-5$ \\
\hline SK-MEL & 0.638 & 2.636 & 2.568 & 1.704 & 1.052 & 0.776 & 0.016 & 97 & 53 & 21 & 7 & -97 & $1.26 \mathrm{E}-7$ & $1.16 \mathrm{E}-5$ & $3.51 \mathrm{E}-5$ \\
\hline UACC- 257 & 0.623 & 1.632 & 1.559 & 1.281 & 1.219 & 0.918 & 0.168 & 93 & 65 & 59 & 29 & -73 & $2.01 \mathrm{E}-6$ & $1.93 \mathrm{E}-5$ & $5.95 \mathrm{E}-5$ \\
\hline UACC- 62 & 0.922 & 2.737 & 2.607 & 1.816 & 1.534 & 1.166 & 0.038 & 93 & 49 & 34 & 13 & -96 & $9.60 \mathrm{E}-8$ & $1.33 \mathrm{E}-5$ & 3. $80 \mathrm{E}-5$ \\
\hline Ovarian Cancer & & & & & & & & & & & & & & & \\
\hline & 0.425 & 1.803 & 1.774 & 1.278 & 0.849 & 0.594 & 0.074 & 98 & 62 & 31 & 12 & -83 & $2.41 \mathrm{E}-7$ & $1.35 \mathrm{E}-5$ & $4.53 \mathrm{E}-5$ \\
\hline OI & 0.770 & 2.012 & 2.085 & 1.188 & 0.876 & 0.838 & 0.041 & 106 & 34 & 9 & 5 & -95 & & $1.13 \mathrm{E}-5$ & $3.58 \mathrm{E}-5$ \\
\hline & 0.704 & 1.710 & 1.704 & 1.456 & 1.236 & 0.939 & 0.249 & 99 & 75 & 53 & 23 & -65 & & & $6.81 \mathrm{E}-5$ \\
\hline & 0.494 & 1.386 & 1.313 & 1.257 & 0.851 & 0.647 & 0.0 & 92 & 86 & 40 & 17 & -94 & & & 4.0 \\
\hline & 0.419 & 2.140 & 2.1 & 1.83 & 1.004 & 0.65 & 0.1 & 100 & 82 & 34 & 14 & -5 & & & $9.09 \mathrm{E}-5$ \\
\hline NCI/ADR-RES & 0.420 & 1.512 & 1.458 & 1.044 & 0.649 & 0.538 & 0.179 & 95 & 57 & 21 & 11 & -57 & & & $7.79 \mathrm{E}-5$ \\
\hline SK-OV-3 & 0.860 & 1.806 & 1.784 & 1.600 & 1.300 & 1.225 & 0.118 & 98 & 78 & 46 & 39 & -86 & $7.73 \mathrm{E}-7$ & $2.04 \mathrm{E}-5$ & $5.12 \mathrm{E}-5$ \\
\hline Renal Cancer & & & & & & & & & & & & & & & \\
\hline $786-0$ & 0.738 & 2.734 & 2.638 & 2.387 & 1.433 & 1.232 & 0.188 & 95 & 83 & 35 & 25 & -75 & $4.81 \mathrm{E}-7$ & $1.77 \mathrm{E}-5$ & $5.65 E-5$ \\
\hline & 1.682 & 2.243 & 2.190 & 1.861 & 1.482 & 1.434 & 0.04 & 91 & 32 & -12 & -15 & -97 & 4.91 & $5.34 \mathrm{E}-7$ & 2.67E-5 \\
\hline $\mathrm{ACHN}$ & 0.3 & 1.223 & 1.25 & 1.003 & 0.649 & 0.484 & 0.00 & 103 & 76 & 38 & 20 & -99 & 4.81 & $1.47 \mathrm{E}-5$ & $3.86 \mathrm{E}-5$ \\
\hline CAKI-1 & 0.78 & 2.278 & 2.0 & 1.453 & 1.223 & 1.19 & 0.04 & 85 & 45 & 29 & 28 & -95 & 7.40E-8 & $1.68 \mathrm{E}-5$ & $4.31 \mathrm{E}-5$ \\
\hline RXF 393 & 1.01 & 1.813 & 1.73 & 1.535 & 1.071 & $\begin{array}{l}1.180 \\
\text {. }\end{array}$ & 0.27 & 90 & 65 & 7 & 20 & -73 & $1.81 \mathrm{E}-7$ & $1.65 \mathrm{E}-5$ & $5.64 \mathrm{E}-5$ \\
\hline SN12C & 1.23 & 3.276 & 3.21 & 3.072 & 2.308 & 1.665 & 0.051 & 97 & 90 & 53 & 21 & -96 & $1.21 \mathrm{E}-6$ & $1.51 \mathrm{E}-5$ & $4.05 \mathrm{E}-5$ \\
\hline TK-10 & 0.872 & 1.725 & 1.615 & 1.519 & 1.290 & 1.114 & 0.135 & 87 & 76 & 49 & 28 & -85 & $9.13 \mathrm{E}-7$ & $1.78 \mathrm{E}-5$ & $4.95 \mathrm{E}-5$ \\
\hline บO-31 & 0.903 & 1.967 & 1.747 & 1.641 & 1.421 & 1.126 & 0.068 & 79 & 69 & 49 & 21 & -93 & 8.60E-7 & $1.53 \mathrm{E}-5$ & $4.22 \mathrm{E}-5$ \\
\hline Prostate Cancer & & & & & & & & & & & & & & & \\
\hline & 0.550 & 1.970 & 1.812 & 1.469 & 0.928 & 0.833 & 0.086 & 89 & 65 & 27 & 20 & -84 & $2.43 \mathrm{E}-7$ & $1.55 \mathrm{E}-5$ & $4.68 \mathrm{E}-5$ \\
\hline DU-145 & 0.429 & 1.662 & 1.747 & 1.528 & 0.763 & 0.638 & 0.145 & 107 & 89 & 27 & 17 & -66 & 4.27E-7 & $1.60 \mathrm{E}-5$ & $6.39 E-5$ \\
\hline Breast Cancer & & & & & & & & & & & & & & & \\
\hline MCF7 & 0.264 & 1.405 & 1.321 & 0.663 & 0.427 & & 0.066 & & & 14 & 7 & -75 & 5.48 & $1.21 \mathrm{E}-5$ & 4.93E-5 \\
\hline MDA-MB-2 & 0.704 & 1.587 & 1.552 & $\begin{array}{l}.005 \\
1.457\end{array}$ & $\begin{array}{l}1.4272 \\
1.072\end{array}$ & 0.780 & 0.119 & 96 & 85 & 42 & 9 & -83 & $\begin{array}{l}6.44 \mathrm{E}-7 \\
6\end{array}$ & 1.24E-5 & 4.35E-5 \\
\hline HS 578T & 1.536 & 2.611 & 2.547 & 2.297 & 1.623 & 1.587 & 0.753 & 94 & 71 & 8 & 5 & -51 & $2.14 \mathrm{E}-7$ & $1.22 \mathrm{E}-5$ & $9.59 \mathrm{E}-5$ \\
\hline BT-549 & 1.118 & 2.356 & 2.267 & 2.011 & 1.703 & 1.459 & 0.145 & 93 & 72 & 47 & 27 & -87 & $7.72 E-7$ & $1.74 \mathrm{E}-5$ & $4.75 E-5$ \\
\hline $\mathrm{T}-47 \mathrm{D}$ & 0.832 & 1.952 & 1.897 & 1.635 & 1.185 & 1.221 & 0.384 & 95 & 72 & 32 & 35 & -54 & $3.47 \mathrm{E}-7$ & $2.47 \mathrm{E}-5$ & $9.05 E-5$ \\
\hline
\end{tabular}


In-Vitro Testing Results

\begin{tabular}{|l|l|l|l|}
\hline NSC : D - 823374 / 1 & Experiment ID : 2008NS40 & Test Type : 08 & Units : Molar \\
\hline Report Date : March 24, 2021 & Test Date : August 24, 2020 & QNS : & MC: \\
\hline COMI : KLB2-21 & Stain Reagent : SRB Dual-Pass Related & SSPL : 1C1X & \\
\hline
\end{tabular}

Log10 Concentration

\begin{tabular}{|c|c|c|c|c|c|c|c|c|c|c|c|c|c|c|c|}
\hline \multirow[b]{2}{*}{ Panel/Cell Line } & \multirow{2}{*}{ Time } & \multirow[b]{2}{*}{ Ctrl } & \multicolumn{5}{|c|}{ Mean Optical Densities } & \multicolumn{5}{|c|}{ Percent Growth } & \multirow[b]{2}{*}{ GI50 } & & \\
\hline & & & -8.0 & -7.0 & -6.0 & -5.0 & -4.0 & -8.0 & -7.0 & -6.0 & -5.0 & -4.0 & & TGI & LC50 \\
\hline Leukemia & & & & & & & & & & & & & & & \\
\hline CCRF-CEM & 0.354 & 1.491 & 1.501 & 1.469 & 0.532 & 0.490 & 0.266 & 101 & 98 & 16 & 12 & -25 & 3.83E-7 & $2.11 \mathrm{E}-5$ & $>1.00 \mathrm{E}-4$ \\
\hline HL-60(TB) & 0.732 & 2.828 & 2.551 & 2.704 & 0.746 & 0.723 & 0.411 & 87 & 94 & 1 & -1 & -44 & 2.96E-7 & $2.15 \mathrm{E}-6$ & $>1.00 \mathrm{E}-4$ \\
\hline K-562 & 0.198 & 1.702 & 1.751 & 1.507 & 0.408 & 0.317 & 0.206 & 103 & 87 & 14 & 8 & 1 & $3.21 \mathrm{E}-7$ & $>1.00 \mathrm{E}-4$ & $>1.00 \mathrm{E}-4$ \\
\hline MOLT-4 & 0.633 & 2.701 & 2.657 & 2.754 & 1.433 & 0.901 & 0.513 & 98 & 103 & 39 & 13 & -19 & $6.65 \mathrm{E}-7$ & $2.54 \mathrm{E}-5$ & $>1.00 \mathrm{E}-4$ \\
\hline RPMI-8226 & 0.988 & 2.284 & 2.364 & 2.351 & 1.269 & 1.235 & 0.903 & 106 & 105 & 22 & 19 & -9 & $4.58 \mathrm{E}-7$ & $4.89 \mathrm{E}-5$ & $>1.00 \mathrm{E}-4$ \\
\hline SR & 0.289 & 1.753 & 1.537 & 1.588 & 0.553 & 0.581 & 0.293 & 85 & 89 & 18 & 20 & . & $3.53 \mathrm{E}-7$ & $>1.00 \mathrm{E}-4$ & $>1.00 \mathrm{E}-4$ \\
\hline Non-Small Cell Lung & Cancer & & & & & & & & & & & & & & \\
\hline A549/ATCC & 0.481 & 2.369 & 2.295 & 2.274 & 1.331 & 0.856 & 0.186 & 96 & 95 & 45 & 20 & -61 & 7.94E-7 & $1.76 \mathrm{E}-5$ & $7.23 E-5$ \\
\hline EKVX & 0.645 & 1.926 & 1.901 & 1.806 & 1.393 & 1.045 & 0.305 & 98 & 91 & 58 & 31 & -53 & $2.03 E-6$ & $2.35 \mathrm{E}-5$ & $9.26 \mathrm{E}-5$ \\
\hline HOP-62 & 1.021 & 2.438 & 2.340 & 2.254 & 1.573 & 1.478 & 0.242 & 93 & 87 & 39 & 32 & -76 & $5.89 \mathrm{E}-7$ & $1.98 \mathrm{E}-5$ & $5.72 \mathrm{E}-5$ \\
\hline HOP-92 & 1.294 & 1.971 & 1.885 & 1.804 & 1.563 & 1.316 & 0.137 & 87 & 75 & 40 & 3 & -89 & $5.13 \mathrm{E}-7$ & $1.08 \mathrm{E}-5$ & $3.75 E-5$ \\
\hline $\mathrm{NCl}-\mathrm{H} 226$ & 1.250 & 2.862 & 2.784 & 2.838 & 1.882 & 1.589 & 0.328 & 95 & 99 & 39 & 21 & -74 & $6.58 \mathrm{E}-7$ & $1.67 \mathrm{E}-5$ & $5.61 \mathrm{E}-5$ \\
\hline $\mathrm{NCl}-\mathrm{H} 23$ & 0.659 & 1.843 & 1.780 & 1.755 & 1.392 & 0.971 & 0.157 & 95 & 93 & 62 & 26 & -76 & 2.16E-6 & $1.81 \mathrm{E}-5$ & $5.55 \mathrm{E}-5$ \\
\hline $\mathrm{NCl}-\mathrm{H} 322 \mathrm{M}$ & 0.932 & 2.287 & 2.205 & 2.170 & 1.712 & 1.605 & 0.093 & 94 & 91 & 58 & 50 & -90 & 8.96E-6 & $2.27 \mathrm{E}-5$ & $5.17 \mathrm{E}-5$ \\
\hline $\mathrm{NCl}-\mathrm{H} 460$ & 0.543 & 3.298 & 3.311 & 3.326 & 1.176 & 0.869 & 0.160 & 100 & 101 & 23 & 12 & -71 & $4.50 \mathrm{E}-7$ & $1.39 \mathrm{E}-5$ & $5.62 \mathrm{E}-5$ \\
\hline $\mathrm{NCl}-\mathrm{H} 522$ & 0.960 & 2.429 & 2.272 & 2.101 & 1.080 & 1.013 & 0.261 & 89 & 78 & 8 & 4 & -73 & $2.50 \mathrm{E}-7$ & $1.11 \mathrm{E}-5$ & $5.03 \mathrm{E}-5$ \\
\hline Colon Cancer & & & & & & & & & & & & & & & \\
\hline COLO 205 & 0.420 & 1.347 & 1.352 & 1.313 & 0.810 & 0.425 & 0.144 & 101 & 96 & 42 & 1 & -66 & 7.13E-7 & $1.02 \mathrm{E}-5$ & $5.77 \mathrm{E}-5$ \\
\hline HCC-2998 & 0.664 & 1.943 & 1.851 & 1.811 & 1.484 & 0.785 & 0.112 & 93 & 90 & 64 & 9 & -83 & $1.81 \mathrm{E}-6$ & $1.26 \mathrm{E}-5$ & $4.38 \mathrm{E}-5$ \\
\hline НСТ-116 & 0.188 & 2.126 & 2.072 & 1.959 & 0.605 & 0.456 & 0.023 & 97 & 91 & 22 & 14 & -88 & $3.91 \mathrm{E}-7$ & $1.37 \mathrm{E}-5$ & $4.25 \mathrm{E}-5$ \\
\hline HCT-15 & 0.271 & 1.604 & 1.581 & 1.500 & 0.647 & 0.442 & 0.082 & 98 & 92 & 28 & 13 & -70 & $4.56 \mathrm{E}-7$ & $1.43 \mathrm{E}-5$ & $5.74 \mathrm{E}-5$ \\
\hline HT29 & 0.337 & 1.947 & 1.995 & 1.916 & 0.472 & 0.366 & 0.104 & 103 & 98 & 8 & 2 & -69 & 3.43E-7 & $1.06 \mathrm{E}-5$ & $5.37 \mathrm{E}-5$ \\
\hline KM12 & 0.632 & 2.833 & 2.898 & 2.753 & 1.180 & 0.993 & 0.102 & 103 & 96 & 25 & 16 & -84 & 4.45E-7 & $1.46 \mathrm{E}-5$ & $4.59 \mathrm{E}-5$ \\
\hline SW-620 & 0.325 & 2.018 & 1.922 & 1.933 & 0.668 & 0.653 & 0.038 & 94 & 95 & 20 & 19 & -88 & 4.00E-7 & $1.51 \mathrm{E}-5$ & $4.40 \mathrm{E}-5$ \\
\hline CNS Cancer & & & & & & & & & & & & & & & \\
\hline SF-268 & 0.965 & 2.415 & 2.374 & 2.315 & $\begin{array}{l}1.678 \\
1.217\end{array}$ & 1.471 & 0.351 & 97 & 93 & 49 & 35 & -64 & $9.58 \mathrm{E}-7$ & $2.26 \mathrm{E}-5$ & 7.27E-5 \\
\hline SF-295 & 0.871 & 2.910 & 2.738 & 2.711 & 1.347 & 1.036 & 0.047 & 92 & 90 & 23 & 8 & -95 & 3.99E-7 & 1.20E-5 & 3.68E-5 \\
\hline SF-539 & 0.592 & 1.898 & 1.865 & 1.763 & 0.796 & 0.438 & 0.060 & 98 & 90 & 16 & -26 & -90 & 3.43E-7 & 2.37E-6 & 2.37E-5 \\
\hline SNB-19 & 0.471 & 1.736 & 1.660 & 1.678 & 0.880 & 0.740 & 0.052 & 94 & 95 & 32 & 21 & -89 & $5.24 \mathrm{E}-7$ & $1.56 \mathrm{E}-5$ & $4.43 \mathrm{E}-5$ \\
\hline SNB-75 & 1.318 & 2.081 & 1.920 & 1.909 & 1.095 & 1.156 & 0.105 & 79 & 78 & -17 & -12 & -92 & $1.96 \mathrm{E}-7$ & $6.62 \mathrm{E}-7$ & $2.97 \mathrm{E}-5$ \\
\hline U251 & 0.341 & 1.733 & 1.652 & 1.583 & 0.769 & 0.553 & 0.061 & 94 & 89 & 31 & 15 & -82 & 4.69E-7 & $1.43 E-5$ & 4.67E-5 \\
\hline Melanoma & & & & & & & & & & & & & & & \\
\hline LOX IMVI & 0.220 & 1.527 & 1.470 & 1.397 & 0.705 & 0.575 & 0.025 & 96 & 90 & 37 & 27 & -89 & $5.70 \mathrm{E}-7$ & $1.71 \mathrm{E}-5$ & $4.62 \mathrm{E}-5$ \\
\hline MALME-3M & 0.598 & 1.048 & 1.000 & 0.952 & 0.798 & 0.823 & 0.088 & 89 & 79 & 44 & 50 & -85 & & $2.34 \mathrm{E}-5$ & $5.49 \mathrm{E}-5$ \\
\hline M14 & 0.642 & 2.172 & 2.128 & 1.955 & 1.076 & 0.837 & 0.106 & 97 & 86 & 28 & 13 & -83 & $4.20 \mathrm{E}-7$ & $1.36 \mathrm{E}-5$ & $4.49 \mathrm{E}-5$ \\
\hline MDA-MB-435 & 0.697 & 2.563 & 2.435 & 2.039 & 0.518 & 0.485 & 0.016 & 93 & 72 & -26 & -30 & -98 & $1.68 \mathrm{E}-7$ & $5.46 \mathrm{E}-7$ & $1.95 \mathrm{E}-5$ \\
\hline SK-MEL-2 & 0.948 & 1.832 & 1.824 & 1.795 & 1.409 & 1.444 & 0.313 & 99 & 96 & 52 & 56 & -67 & $1.12 \mathrm{E}-5$ & $2.86 \mathrm{E}-5$ & $7.28 \mathrm{E}-5$ \\
\hline SK-MEL-28 & 0.770 & 1.937 & 1.979 & 1.843 & 1.499 & 1.351 & 0.128 & 104 & 92 & 62 & 50 & -83 & $9.62 \mathrm{E}-6$ & $2.37 \mathrm{E}-5$ & $5.62 \mathrm{E}-5$ \\
\hline SK-MEL-5 & 0.638 & 2.618 & 2.574 & 2.457 & 1.203 & 0.983 & 0.004 & 98 & 92 & 29 & 17 & -99 & $4.58 \mathrm{E}-7$ & $1.41 \mathrm{E}-5$ & $3.78 \mathrm{E}-5$ \\
\hline UACC-257 & 0.623 & 1.630 & 1.538 & 1.500 & 1.079 & 1.172 & 0.199 & 91 & 87 & 45 & 55 & -68 & & $2.79 \mathrm{E}-5$ & $7.12 \mathrm{E}-5$ \\
\hline UACC-62 & 0.922 & 2.786 & 2.685 & 2.505 & 1.509 & 1.269 & 0.035 & 95 & 85 & 31 & 19 & -96 & $4.50 \mathrm{E}-7$ & $1.45 \mathrm{E}-5$ & $3.96 \mathrm{E}-5$ \\
\hline Ovarian Cancer & & & & & & & & & & & & & & & \\
\hline IGROV1 & 0.425 & 1.830 & 1.767 & 1.726 & 0.884 & 0.638 & 0.092 & 96 & 93 & 33 & 15 & -78 & $5.14 \mathrm{E}-7$ & $1.45 \mathrm{E}-5$ & $4.97 \mathrm{E}-5$ \\
\hline OVCAR-3 & 0.770 & 2.001 & 2.098 & 2.001 & 0.891 & 0.732 & 0.068 & 108 & 100 & 10 & -5 & -91 & $3.58 \mathrm{E}-7$ & $4.59 \mathrm{E}-6$ & $3.33 E-5$ \\
\hline OVCAR-4 & 0.704 & 1.679 & 1.701 & 1.618 & 1.221 & 0.970 & 0.191 & 102 & 94 & 53 & 27 & -73 & $1.31 \mathrm{E}-6$ & $1.87 \mathrm{E}-5$ & $5.91 \mathrm{E}-5$ \\
\hline OVCAR-5 & 0.494 & 1.321 & 1.261 & 1.238 & 0.988 & 0.566 & 0.030 & 93 & 90 & 60 & 9 & -94 & $1.55 \mathrm{E}-6$ & $1.22 \mathrm{E}-5$ & $3.73 E-5$ \\
\hline OVCAR-8 & 0.419 & 2.172 & 2.091 & 2.055 & 1.331 & 0.788 & 0.202 & 95 & 93 & 52 & 21 & -52 & $1.16 \mathrm{E}-6$ & $1.94 \mathrm{E}-5$ & $9.42 \mathrm{E}-5$ \\
\hline NCI/ADR-RES & 0.420 & 1.438 & 1.437 & 1.399 & 0.744 & 0.581 & 0.136 & 100 & 96 & 32 & 16 & -68 & $5.21 \mathrm{E}-7$ & $1.55 \mathrm{E}-5$ & $6.15 \mathrm{E}-5$ \\
\hline SK-OV-3 & 0.860 & 1.775 & 1.778 & 1.753 & 1.296 & 1.206 & 0.263 & 100 & 98 & 48 & 38 & -69 & 8.96E-7 & $2.25 \mathrm{E}-5$ & $6.58 \mathrm{E}-5$ \\
\hline Renal Cancer & & & & & & & & & & & & & & & \\
\hline $786-0$ & 0.738 & 2.721 & 2.621 & 2.454 & 1.293 & 1.233 & 0.136 & 95 & 87 & 28 & 25 & -82 & $4.20 \mathrm{E}-7$ & $1.71 \mathrm{E}-5$ & $5.05 E-5$ \\
\hline A498 & 1.682 & 2.216 & 2.204 & 2.043 & 1.562 & 1.518 & 0.062 & 98 & 68 & -7 & -10 & -96 & $1.72 \mathrm{E}-7$ & $8.02 \mathrm{E}-7$ & $2.92 \mathrm{E}-5$ \\
\hline ACHN & 0.300 & 1.188 & 1.184 & 1.146 & 0.585 & 0.505 & 0.071 & 100 & 95 & 32 & 23 & -77 & $5.21 \mathrm{E}-7$ & $1.70 \mathrm{E}-5$ & $5.42 \mathrm{E}-5$ \\
\hline CAKI-1 & 0.784 & 2.265 & 2.065 & 1.931 & 1.269 & 1.193 & 0.034 & 86 & 77 & 33 & 28 & -96 & $4.11 \mathrm{E}-7$ & $1.67 \mathrm{E}-5$ & $4.26 \mathrm{E}-5$ \\
\hline RXF 393 & 1.018 & 1.718 & 1.702 & 1.716 & 1.290 & 1.136 & 0.249 & 98 & 100 & 39 & 17 & -76 & $6.56 \mathrm{E}-7$ & $1.52 \mathrm{E}-5$ & $5.29 \mathrm{E}-5$ \\
\hline SN12C & 1.234 & 3.285 & 3.200 & 3.195 & 2.536 & 1.823 & 0.051 & 96 & 96 & 63 & 29 & -96 & 2.44E-6 & $1.70 \mathrm{E}-5$ & $4.28 \mathrm{E}-5$ \\
\hline TK-10 & 0.872 & 1.726 & 1.644 & 1.568 & 1.408 & 1.214 & 0.221 & 90 & 82 & 63 & 40 & -75 & 3.64E-6 & $2.23 \mathrm{E}-5$ & $6.09 \mathrm{E}-5$ \\
\hline UO-31 & 0.903 & 1.992 & 1.773 & 1.801 & 1.477 & 1.173 & 0.037 & 80 & 82 & 53 & 25 & -96 & $1.25 \mathrm{E}-6$ & $1.60 \mathrm{E}-5$ & $4.16 \mathrm{E}-5$ \\
\hline Prostate Cancer & & & & & & & & & & & & & & & \\
\hline PC-3 & 0.550 & 1.813 & 1.740 & 1.715 & 1.002 & 0.775 & 0.089 & 94 & 92 & 36 & 18 & -84 & $5.60 \mathrm{E}-7$ & $1.50 \mathrm{E}-5$ & 4.64E-5 \\
\hline DU-145 & 0.429 & 1.617 & 1.665 & 1.652 & 1.130 & 0.637 & 0.169 & 104 & 103 & 59 & 18 & -61 & $1.64 \mathrm{E}-6$ & $1.68 \mathrm{E}-5$ & $7.32 \mathrm{E}-5$ \\
\hline Breast Cancer & & & & & & & & & & & & & & & \\
\hline MCF7 & 0.264 & 1.420 & 1.337 & 1.309 & 0.404 & 0.368 & 0.140 & 93 & 90 & 12 & 9 & -47 & $3.28 \mathrm{E}-7$ & $1.45 \mathrm{E}-5$ & $>1.00 \mathrm{E}-4$ \\
\hline MDA-MB-231/ATCC & 0.704 & 1.593 & 1.561 & 1.532 & 1.264 & 0.841 & 0.129 & 96 & 93 & 63 & 15 & -82 & $1.88 \mathrm{E}-6$ & $1.44 \mathrm{E}-5$ & $4.72 \mathrm{E}-5$ \\
\hline HS 578T & 1.536 & 2.553 & 2.539 & 2.501 & 1.673 & 1.702 & 0.774 & 99 & 95 & 13 & 16 & -50 & 3.56E-7 & $1.77 \mathrm{E}-5$ & $>1.00 \mathrm{E}-4$ \\
\hline BT-549 & 1.118 & 2.367 & 2.360 & 2.229 & 1.634 & 1.479 & 0.129 & 99 & 89 & 41 & 29 & -89 & $6.56 \mathrm{E}-7$ & $1.76 \mathrm{E}-5$ & 4.70E-5 \\
\hline T-47D & 0.832 & 1.910 & 1.850 & 1.801 & 1.149 & 1.125 & 0.417 & 94 & 90 & 29 & 27 & -50 & 4.57E-7 & $2.25 \mathrm{E}-5$ & $>1.00 \mathrm{E}-4$ \\
\hline
\end{tabular}


In-Vitro Testing Results

\begin{tabular}{|l|l|l|l|}
\hline NSC : D - 823375/1 & Experiment ID : 2008NS40 & Test Type : 08 & Units : Molar \\
\hline Report Date : March 24, 2021 & Test Date : August 24, 2020 & QNS : & MC : \\
\hline COMI : KLB2-22 & Stain Reagent : SRB Dual-Pass Related & SSPL : 1C1X & \\
\hline
\end{tabular}

Log10 Concentration

\begin{tabular}{|c|c|c|c|c|c|c|c|c|c|c|c|c|c|c|c|}
\hline \multirow[b]{2}{*}{ Panel/Cell Line } & \multirow{2}{*}{$\begin{array}{l}\text { Time } \\
\text { Zero }\end{array}$} & \multirow[b]{2}{*}{ Ctrl } & \multicolumn{5}{|c|}{ Mean Optical Densities } & \multicolumn{5}{|c|}{ Percent Growth } & \multirow[b]{2}{*}{ G150 } & & \\
\hline & & & -8.0 & -7.0 & -6.0 & -5.0 & -4.0 & -8.0 & -7.0 & -6.0 & -5.0 & -4.0 & & TGI & LC50 \\
\hline Leukemia & & & & & & & & & & & & & & & \\
\hline CCRF-CEM & 0.354 & 1.494 & 1.228 & 1.451 & 0.516 & 0.430 & 0.335 & 77 & 96 & 14 & 7 & -6 & 3.66E-7 & $3.52 \mathrm{E}-5$ & $>1.00 \mathrm{E}-4$ \\
\hline HL-60(TB) & 0.732 & 2.670 & 2.322 & 2.312 & 0.834 & 0.538 & 0.431 & 82 & 82 & 5 & -27 & -41 & 2.59E-7 & $1.46 \mathrm{E}-6$ & $>1.00 \mathrm{E}-4$ \\
\hline K-562 & 0.198 & 1.811 & 0.713 & 0.991 & 0.369 & 0.256 & 0.195 & 32 & 49 & 11 & 4 & -2 & $<1.00 \mathrm{E}-8$ & $4.68 \mathrm{E}-5$ & $>1.00 \mathrm{E}-4$ \\
\hline MOLT-4 & 0.633 & 2.764 & 2.451 & 2.911 & 1.301 & 0.715 & 0.603 & 85 & 107 & 31 & 4 & -5 & 5.66E-7 & $2.78 \mathrm{E}-5$ & $>1.00 \mathrm{E}-4$ \\
\hline RPMI-8226 & 0.988 & 2.370 & 2.242 & 2.302 & 1.311 & 0.934 & 0.668 & 91 & 95 & 23 & -6 & -32 & 4.25E-7 & $6.44 \mathrm{E}-6$ & $>1.00 \mathrm{E}-4$ \\
\hline SR & 0.289 & 1.691 & 0.896 & 1.221 & 0.510 & 0.309 & 0.222 & 43 & 67 & 16 & 1 & -23 & & $1.14 \mathrm{E}-5$ & $>1.00 \mathrm{E}-4$ \\
\hline Non-Small Cell Lung & Cancer & & & & & & & & & & & & & & \\
\hline A549/ATCC & 0.481 & 2.278 & 1.941 & 2.205 & 1.222 & 0.551 & 0.451 & 81 & 96 & 41 & 4 & -6 & $6.91 \mathrm{E}-7$ & $2.42 \mathrm{E}-5$ & $>1.00 \mathrm{E}-4$ \\
\hline EKVX & 0.645 & 1.853 & 1.698 & 1.770 & 1.126 & 0.672 & 0.614 & 87 & 93 & 40 & 2 & -5 & $6.43 \mathrm{E}-7$ & $2.06 \mathrm{E}-5$ & $>1.00 \mathrm{E}-4$ \\
\hline HOP-62 & 1.021 & 2.334 & 2.013 & 2.161 & 1.455 & 1.176 & 0.558 & 76 & 87 & 33 & 12 & -45 & 4.84E-7 & $1.61 \mathrm{E}-5$ & $>1.00 \mathrm{E}-4$ \\
\hline HOP-92 & 1.294 & 1.965 & 1.758 & 1.884 & 1.516 & 1.051 & 0.772 & 69 & 88 & 33 & -19 & -40 & $4.90 \mathrm{E}-7$ & 4.34E-6 & $>1.00 \mathrm{E}-4$ \\
\hline $\mathrm{NCl}-\mathrm{H} 226$ & 1.250 & 2.862 & 2.615 & 2.838 & 1.758 & 1.210 & 0.532 & 85 & 98 & 32 & -3 & -57 & 5.30E-7 & 8.07E-6 & $7.28 \mathrm{E}-5$ \\
\hline $\mathrm{NCl}-\mathrm{H} 23$ & 0.659 & 1.850 & 1.719 & 1.827 & 1.353 & 0.859 & 0.559 & 89 & 98 & 58 & 17 & -15 & $1.58 \mathrm{E}-6$ & 3.34E-5 & $>1.00 \mathrm{E}-4$ \\
\hline $\mathrm{NCl}-\mathrm{H} 322 \mathrm{M}$ & 0.932 & 2.291 & 2.211 & 2.197 & 1.621 & 1.403 & 0.878 & 94 & 93 & 51 & 35 & -6 & $1.11 \mathrm{E}-6$ & 7.19E-5 & $>1.00 \mathrm{E}-4$ \\
\hline $\mathrm{NCl}-\mathrm{H} 460$ & 0.543 & 3.331 & 3.280 & 3.327 & 1.290 & 0.603 & 0.362 & 98 & 100 & 27 & 2 & -33 & $4.81 \mathrm{E}-7$ & 1.15E-5 & $>1.00 \mathrm{E}-4$ \\
\hline NCl-H522 & 0.960 & 2.318 & 1.752 & 1.987 & 1.173 & 0.819 & 0.560 & 58 & 76 & 16 & -15 & -42 & 2.67E-7 & $3.28 \mathrm{E}-6$ & $>1.00 \mathrm{E}-4$ \\
\hline Colon Cancer & & & & & & & & & & & & & & & \\
\hline COLO 205 & 0.420 & 1.182 & $\begin{array}{l}1.099 \\
1.689\end{array}$ & 1.193 & 0.605 & 0.138 & 0.086 & 89 & 101 & 24 & -67 & -80 & 4.64E-7 & 1.84E-6 & 6.48E-6 \\
\hline HCC-2998 & 0.664 & 1.883 & 1.689 & 1.866 & 1.414 & 0.677 & 0.440 & 84 & 99 & 62 & 1 & -34 & 1.55E-6 & $1.07 \mathrm{E}-5$ & $>1.00 \mathrm{E}-4$ \\
\hline HCT-116 & 0.188 & 2.007 & 1.638 & 1.868 & 0.574 & 0.223 & 0.085 & 80 & 92 & 21 & 2 & -55 & 3.94E-7 & $1.08 \mathrm{E}-5$ & $8.15 \mathrm{E}-5$ \\
\hline HCT-15 & 0.271 & 1.476 & 1.023 & 1.303 & 0.495 & 0.290 & 0.172 & 62 & 86 & 19 & 2 & -37 & 3.40E-7 & 1.10E-5 & $>1.00 \mathrm{E}-4$ \\
\hline HT29 & 0.337 & 1.891 & 1.568 & 1.890 & 0.395 & 0.286 & 0.152 & 79 & 100 & 4 & -15 & -55 & 3.30E-7 & $1.57 \mathrm{E}-6$ & $7.53 \mathrm{E}-5$ \\
\hline KM12 & 0.632 & 2.913 & 2.043 & 2.297 & 0.995 & 0.670 & 0.250 & 62 & 73 & 16 & 2 & -60 & $2.53 \mathrm{E}-7$ & 1.06E-5 & $6.79 \mathrm{E}-5$ \\
\hline SW-620 & 0.325 & 2.084 & 1.558 & 1.883 & 0.647 & 0.607 & 0.305 & 70 & 89 & 18 & 16 & -6 & $3.54 \mathrm{E}-7$ & $5.22 \mathrm{E}-5$ & $>1.00 \mathrm{E}-4$ \\
\hline CNS Cancer & & & & & & & & & & & & & & & \\
\hline SF-268 & 0.965 & $\begin{array}{r}2.310 \\
2902\end{array}$ & 2.138 & 2.204 & 1.582 & 1.009 & 0.757 & 87 & 92 & 46 & 3 & -22 & $8.12 \mathrm{E}-7$ & 1.35E-5 & $>1.00 \mathrm{E}-4$ \\
\hline SF-295 & 0.871 & 2.902 & $\begin{array}{l}2.414 \\
1730\end{array}$ & 2.689 & 1.373 & 0.688 & 0.157 & 76 & 89 & 25 & -21 & -82 & 4.07E-7 & 3.47E-6 & $2.99 \mathrm{E}-5$ \\
\hline SF-539 & 0.592 & 1.918 & 1.739 & 1.871 & 0.778 & 0.340 & 0.192 & 86 & 96 & 14 & -43 & -68 & 3.66E-7 & 1.77E-6 & $1.98 \mathrm{E}-5$ \\
\hline SNB-19 & 0.471 & 1.731 & 1.541 & 1.657 & 0.828 & 0.657 & 0.427 & 85 & 94 & 28 & 15 & -9 & $4.69 \mathrm{E}-7$ & 4.07E-5 & $>1.00 \mathrm{E}-4$ \\
\hline SNB-75 & 1.318 & 2.023 & 1.619 & 1.783 & 0.957 & 0.802 & 0.760 & 43 & 66 & -27 & -39 & -42 & & 5.09E-7 & $>1.00 \mathrm{E}-4$ \\
\hline U251 & 0.341 & 1.626 & 1.446 & 1.563 & 0.640 & 0.381 & 0.179 & 86 & 95 & 23 & 3 & -48 & 4.24E-7 & 1.15E-5 & $>1.00 \mathrm{E}-4$ \\
\hline Melanoma & & & & & & & & & & & & & & & \\
\hline LOX IMVI & 0.220 & 1.481 & 1.231 & 1.373 & 0.633 & 0.239 & 0.056 & 80 & 91 & 33 & 1 & -75 & 5.09E-7 & 1.05E-5 & $4.73 \mathrm{E}-5$ \\
\hline MALME & 0.598 & 1.051 & 0.943 & 0.994 & 0.791 & 0.659 & 0.367 & 76 & 87 & 43 & 13 & -39 & $6.83 \mathrm{E}-7$ & $1.81 \mathrm{E}-5$ & $>1.00 \mathrm{E}-4$ \\
\hline & 0.642 & 2.254 & 1.848 & 2.068 & 0.988 & 0.703 & 0.329 & 75 & 88 & 21 & 4 & -49 & $3.75 \mathrm{E}-7$ & $1.18 \mathrm{E}-5$ & $>1.00 \mathrm{E}-4$ \\
\hline MDA-MB-435 & 0.697 & 2.756 & 0.931 & 1.475 & 0.548 & 0.542 & 0.353 & 11 & 38 & -21 & -22 & -49 & $<1.00 \mathrm{E}-8$ & $4.34 \mathrm{E}-7$ & $>1.00 \mathrm{E}-4$ \\
\hline SK-MEL-2 & 0.948 & 1.754 & 1.681 & 1.738 & 1.309 & 1.039 & 0.382 & 91 & 98 & 45 & 11 & -60 & 7.97E-7 & $1.44 \mathrm{E}-5$ & $7.30 \mathrm{E}-5$ \\
\hline SK-ME & 0.770 & 1.943 & 1.789 & 1.773 & 1.397 & 0.995 & 0.449 & 87 & 85 & 53 & 19 & -42 & 1.26E-6 & $2.06 \mathrm{E}-5$ & $>1.00 \mathrm{E}-4$ \\
\hline SK-ME & 0.638 & 2.759 & 2.250 & 2.523 & 1.129 & 0.146 & 0.052 & 76 & 89 & 23 & -77 & -92 & 3.91E-7 & $1.70 \mathrm{E}$ & $5.37 \mathrm{E}-6$ \\
\hline UACC-257 & 0.623 & 1.561 & 1.413 & 1.456 & 1.061 & 0.617 & 0.269 & 84 & 89 & 47 & & -57 & 8.36E-7 & $9.55 \mathrm{E}-6$ & $7.53 \mathrm{E}-5$ \\
\hline UACC-62 & 0.922 & 2.837 & 2.327 & 2.560 & 1.592 & 1.166 & 0.302 & 73 & 86 & 35 & 13 & -67 & $5.05 \mathrm{E}-7$ & $1.44 \mathrm{E}-5$ & $6.09 E-5$ \\
\hline Ovarian Cancer & & & & & & & & & & & & & & & \\
\hline IGROV1 & 0.425 & 1.808 & 1.534 & 1.686 & 0.885 & 0.600 & 0.282 & 80 & 91 & 33 & 13 & -34 & $5.14 \mathrm{E}-7$ & 1.87E-5 & $>1.00 \mathrm{E}-4$ \\
\hline OVCA & 0.770 & 1.947 & 1.641 & 1.878 & 0.824 & 0.694 & 0.33 & 74 & 94 & 5 & -10 & -57 & 3.11E-7 & $2.06 \mathrm{E}-6$ & $7.12 \mathrm{E}-5$ \\
\hline & 0.70 & 1.620 & 1.499 & 1.583 & 1.145 & 0.705 & 0.6 & 87 & 96 & 48 & ret & -6 & 9.12 & & $>1.00 \mathrm{E}-4$ \\
\hline & 0.4 & 1.305 & 1.235 & 1.268 & 0.882 & 0.536 & 0.3 & 91 & 95 & 48 & 5 & -24 & & & $>1.00 \mathrm{E}-4$ \\
\hline & 0.4 & 1.985 & 1.834 & 2.039 & 1.056 & 0.483 & 0.4 & 90 & 103 & 41 & 4 & -4 & 7.11 & & $>1.00 \mathrm{E}-4$ \\
\hline NCI/ADR-RES & 0.420 & 1.465 & 1.172 & 1.367 & 0.703 & 0.473 & 0.354 & 72 & 91 & 27 & 5 & -16 & 4.36E-7 & & $>1.00 \mathrm{E}-4$ \\
\hline SK-OV-3 & 0.860 & 1.636 & 1.565 & 1.624 & 1.167 & 0.834 & 0.627 & 91 & 99 & 40 & -3 & -27 & $6.66 \mathrm{E}-7$ & $8.49 \mathrm{E}-6$ & $>1.00 \mathrm{E}-4$ \\
\hline Renal Cancer & & & & & & & & & & & & & & & \\
\hline & 0.738 & 2.672 & 2.459 & 2.516 & 1.276 & 0.912 & 0.377 & 89 & 92 & 28 & 9 & -49 & 4.51E-7 & 1.43E-5 & $>1.00 \mathrm{E}-4$ \\
\hline A4 & 1.682 & 2.254 & 2.023 & 2.221 & 1.637 & 1.314 & 0.86 & 60 & 94 & -3 & -22 & & & & $>1.00 \mathrm{E}-4$ \\
\hline ACHN & 0.300 & 1.217 & 1.153 & 1.231 & 0.582 & 0.338 & 0.11 & 93 & 102 & 31 & 4 & -6 & & & $6.52 \mathrm{E}-5$ \\
\hline CAKI & 0.784 & 2.36 & 1.655 & 1.888 & 1.235 & 0.92 & 0.56 & 55 & 70 & 28 & 9 & -2 & & & $>1.00 \mathrm{E}-4$ \\
\hline RXF 393 & 1.018 & 1.713 & 1.540 & 1.616 & 1.183 & 0.910 & 0.38 & 75 & 86 & 24 & -11 & -6 & & 4.91 & $5.84 \mathrm{E}-5$ \\
\hline SN12C & 1.234 & 3.242 & 3.084 & 3.157 & 2.154 & 1.321 & 0.43 & 92 & 96 & 46 & 4 & -6 & & 1.15 & $6.15 \mathrm{E}-5$ \\
\hline TK-1 & 0.872 & 1.672 & 1.54 & 1.586 & 1.293 & 0.81 & 0.58 & 84 & 89 & 53 & -6 & -3 & & & $>1.00 \mathrm{E}-4$ \\
\hline บO-31 & 0.903 & 1.932 & 1.711 & 1.764 & 1.389 & 0.850 & 0.725 & 79 & 84 & 47 & -6 & -20 & 8.39E-7 & 7.74E-6 & $>1.00 \mathrm{E}-4$ \\
\hline Prost & & & & & & & & & & & & & & & \\
\hline & 0.550 & 1.797 & 1.454 & 1.623 & 0.914 & 0.529 & 0.400 & 72 & 86 & 29 & -4 & -27 & 4.30E-7 & 7.62E-6 & $>1.00 \mathrm{E}-4$ \\
\hline DU-145 & 0.429 & 1.619 & 1.602 & 1.701 & 0.947 & 0.544 & 0.409 & 99 & 107 & 44 & 10 & -5 & $7.90 \mathrm{E}-7$ & 4.72E-5 & $>1.00 \mathrm{E}-4$ \\
\hline Breast Ca & & & & & & & & & & & & & & & \\
\hline MCF & 0.264 & 1.323 & 0.89 & 1.123 & 0.368 & 0.231 & 0.167 & 60 & 81 & 10 & -13 & -3 & & & \\
\hline ML & 0.704 & 1.622 & & 1.563 & 1.120 & 0.724 & 0.6 & 94 & 93 & 45 & 2 & & & & \\
\hline HS 57 & 1.536 & 2.549 & 2.27 & 2.516 & 1.828 & 1.370 & 1.0 & 73 & 97 & 29 & -11 & -3 & & & $.00 \mathrm{E}-4$ \\
\hline BT-54C & 1.118 & 2.350 & 2.154 & 2.290 & 1.639 & 1.216 & 0.564 & 84 & 95 & 42 & 8 & $\begin{array}{l}-50 \\
15\end{array}$ & 7.15E-7 & & $>1.00 \mathrm{E}-4$ \\
\hline T-47D & 0.832 & 1.872 & 1.655 & 1.730 & 1.068 & 0.878 & 0.704 & 79 & 86 & 23 & 4 & -15 & $3.73 \mathrm{E}-7$ & 1.67E-5 & $>1.00 \mathrm{E}-4$ \\
\hline
\end{tabular}


In-Vitro Testing Results

\begin{tabular}{|l|l|l|l|}
\hline NSC : D - 823377 / 1 & Experiment ID : 2008NS40 & Test Type : 08 & Units : Molar \\
\hline Report Date : March 24, 2021 & Test Date : August 24, 2020 & QNS : & MC : \\
\hline COMI : KLB2-24 & Stain Reagent : SRB Dual-Pass Related & SSPL : 1C1X & \\
\hline
\end{tabular}

Log10 Concentration

\begin{tabular}{|c|c|c|c|c|c|c|c|c|c|c|c|c|c|c|c|}
\hline \multirow[b]{2}{*}{ Panel/Cell Line } & \multirow{2}{*}{$\begin{array}{l}\text { Time } \\
\text { Zero }\end{array}$} & \multirow[b]{2}{*}{ Ctrl } & \multicolumn{5}{|c|}{ Mean Optical Densities } & \multicolumn{5}{|c|}{ Percent Growth } & \multirow[b]{2}{*}{ G150 } & & \\
\hline & & & -8.0 & -7.0 & -6.0 & -5.0 & -4.0 & -8.0 & -7.0 & -6.0 & -5.0 & -4.0 & & TGI & LC50 \\
\hline Leukemia & & & & & & & & & & & & & & & \\
\hline CCRF-CEM & 0.354 & 1.618 & 1.667 & 1.593 & 0.709 & 0.550 & 0.480 & 104 & 98 & 28 & 16 & 10 & $4.85 \mathrm{E}-7$ & $>1.00 \mathrm{E}-4$ & $>1.00 \mathrm{E}-4$ \\
\hline HL-60(TB) & 0.732 & 2.759 & 2.536 & 2.645 & 0.773 & 0.710 & 0.423 & 89 & 94 & 2 & -3 & -42 & 3.02E-7 & 2.51E-6 & $>1.00 \mathrm{E}-4$ \\
\hline $\mathrm{K}-562$ & 0.198 & 1.758 & 1.813 & 1.078 & 0.381 & 0.319 & 0.210 & 104 & 56 & 12 & 8 & 1 & $1.39 \mathrm{E}-7$ & $>1.00 \mathrm{E}-4$ & $>1.00 \mathrm{E}-4$ \\
\hline MOLT-4 & 0.633 & 2.720 & 2.692 & 2.716 & 1.296 & 1.084 & 0.572 & 99 & 100 & 32 & 22 & -10 & 5.39E-7 & 4.91E-5 & $>1.00 \mathrm{E}-4$ \\
\hline RPMI-8226 & 0.988 & 2.430 & 2.457 & 2.290 & 1.403 & 1.331 & 0.862 & 102 & 90 & 29 & 24 & -13 & 4.52E-7 & 4.47E-5 & $>1.00 \mathrm{E}-4$ \\
\hline & 0.289 & 1.514 & 1.444 & 1.113 & 0.486 & 0.433 & 0.294 & 94 & 67 & 16 & 12 & & 2.17E-7 & $>1.00 \mathrm{E}-4$ & $>1.00 \mathrm{E}-4$ \\
\hline Non-Small Cell Lung & Cancer & & & & & & & & & & & & & & \\
\hline A549/ATCC & 0.481 & 2.436 & 2.355 & 2.297 & 1.348 & 1.078 & 0.533 & 96 & 93 & 44 & 31 & 3 & $7.65 \mathrm{E}-7$ & $>1.00 \mathrm{E}-4$ & $>1.00 \mathrm{E}-4$ \\
\hline EKVX & 0.645 & 1.892 & 1.871 & 1.777 & 1.221 & 0.988 & 0.579 & 98 & 91 & 46 & 27 & -10 & $8.21 \mathrm{E}-7$ & 5.34E-5 & $>1.00 \mathrm{E}-4$ \\
\hline HOP-62 & 1.021 & 2.432 & 2.336 & 2.206 & 1.569 & 1.430 & 0.855 & 93 & 84 & 39 & 29 & -16 & $5.65 \mathrm{E}-7$ & $4.36 \mathrm{E}-5$ & $>1.00 \mathrm{E}-4$ \\
\hline HOP-92 & 1.294 & 2.048 & 1.979 & 1.886 & 1.631 & 1.381 & 0.925 & 91 & 78 & 45 & 12 & -29 & $6.93 \mathrm{E}-7$ & $1.94 \mathrm{E}-5$ & $>1.00 \mathrm{E}-4$ \\
\hline $\mathrm{NCl}-\mathrm{H} 226$ & 1.250 & 2.875 & 2.745 & 2.710 & 1.949 & 1.667 & 0.810 & 92 & 90 & 43 & 26 & -35 & 7.09E-7 & 2.64E-5 & $>1.00 \mathrm{E}-4$ \\
\hline $\mathrm{NCl}-\mathrm{H} 23$ & 0.659 & 1.877 & 1.776 & 1.767 & 1.327 & 1.035 & 0.589 & 92 & 91 & 55 & 31 & -11 & $1.59 \mathrm{E}-6$ & $5.53 \mathrm{E}-5$ & $>1.00 \mathrm{E}-4$ \\
\hline $\mathrm{NCl}-\mathrm{H} 322 \mathrm{M}$ & 0.932 & 2.272 & 2.218 & 2.156 & 1.697 & 1.506 & 0.957 & 96 & 91 & 57 & 43 & 2 & $3.15 \mathrm{E}-6$ & $>1.00 \mathrm{E}-4$ & $>1.00 \mathrm{E}-4$ \\
\hline $\mathrm{NCl}-\mathrm{H} 460$ & 0.543 & 3.334 & 3.318 & 3.316 & 1.143 & 1.020 & 0.424 & 99 & 99 & 22 & 17 & -22 & 4.30E-7 & 2.74E-5 & $>1.00 \mathrm{E}-4$ \\
\hline $\mathrm{NCl}-\mathrm{H} 522$ & 0.960 & 2.444 & 2.320 & 2.077 & 1.246 & 1.084 & 0.687 & 92 & 75 & 19 & 8 & -28 & $2.82 \mathrm{E}-7$ & $1.68 \mathrm{E}-5$ & $>1.00 \mathrm{E}-4$ \\
\hline Colon Cancer & & & & & & & & & & & & & & & \\
\hline COLO 205 & 0.420 & 1.385 & 1.378 & 1.348 & 0.780 & 0.411 & 0.091 & 99 & 96 & 37 & -2 & -78 & $6.08 \mathrm{E}-7$ & $8.82 \mathrm{E}-6$ & $4.25 \mathrm{E}-5$ \\
\hline HCC-2998 & 0.664 & 1.887 & 1.793 & 1.728 & 1.345 & 1.072 & 0.316 & 92 & 87 & 56 & 33 & -52 & $1.80 \mathrm{E}-6$ & $2.45 \mathrm{E}-5$ & $9.36 \mathrm{E}-5$ \\
\hline HCT-116 & 0.188 & 2.066 & 2.068 & 1.959 & 0.555 & 0.595 & 0.218 & 100 & 94 & 20 & 22 & 2 & 3.92E-7 & $>1.00 \mathrm{E}-4$ & $>1.00 \mathrm{E}-4$ \\
\hline HCT-15 & 0.271 & 1.554 & 1.497 & 1.287 & 0.529 & 0.519 & 0.247 & 95 & 79 & 20 & 19 & -9 & $3.12 \mathrm{E}-7$ & $4.85 \mathrm{E}-5$ & $>1.00 \mathrm{E}-4$ \\
\hline HT29 & 0.337 & 2.002 & 2.012 & 1.979 & 0.507 & 0.458 & 0.269 & 101 & 99 & 10 & 7 & -20 & 3.54E-7 & 1.83E-5 & $>1.00 \mathrm{E}-4$ \\
\hline KM12 & 0.632 & 3.065 & 3.024 & 2.547 & 1.437 & 1.256 & 0.358 & 98 & 79 & 33 & 26 & -43 & 4.25E-7 & $2.35 \mathrm{E}-5$ & $>1.00 \mathrm{E}-4$ \\
\hline SW-620 & 0.325 & 2.105 & 2.098 & 1.932 & 0.727 & 0.720 & 0.428 & 100 & 90 & 23 & 22 & 6 & 3.94E-7 & $>1.00 \mathrm{E}-4$ & $>1.00 \mathrm{E}-4$ \\
\hline CNS Cancer & & & & & & & & & & & & & & & \\
\hline SF-268 & 0.965 & 2.472 & 2.439 & 2.310 & 1.748 & 1.640 & 0.776 & 98 & 89 & 52 & 45 & -20 & $1.85 \mathrm{E}-6$ & $4.96 \mathrm{E}-5$ & $>1.00 \mathrm{E}-4$ \\
\hline SF-295 & 0.871 & 2.919 & 2.836 & 2.743 & 1.363 & 1.101 & 0.482 & 96 & 91 & 24 & 11 & -45 & 4.12E-7 & 1.59E-5 & $>1.00 \mathrm{E}-4$ \\
\hline SF-539 & 0.592 & 1.881 & 1.818 & 1.732 & 0.880 & 0.426 & 0.269 & 95 & 88 & 22 & -28 & -55 & 3.81E-7 & 2.77E- 6 & $6.72 \mathrm{E}-5$ \\
\hline SNB-19 & 0.471 & 1.730 & 1.636 & 1.578 & 0.817 & 0.732 & 0.494 & 93 & 88 & 27 & 21 & 2 & 4.24E-7 & $>1.00 \mathrm{E}-4$ & $>1.00 \mathrm{E}-4$ \\
\hline SNB-75 & 1.318 & 2.263 & 2.110 & 2.006 & 1.176 & 0.864 & 0.822 & 84 & 73 & -11 & -34 & -38 & $1.88 \mathrm{E}-7$ & 7.43E-7 & $>1.00 \mathrm{E}-4$ \\
\hline U251 & 0.341 & 1.729 & 1.659 & 1.555 & 0.752 & 0.624 & 0.270 & 95 & 87 & 30 & 20 & -21 & 4.44E-7 & 3.12E-5 & $>1.00 \mathrm{E}-4$ \\
\hline Melanoma & & & & & & & & & & & & & & & \\
\hline LOX IM & 0.220 & 1.506 & 1.486 & 1.323 & 0.618 & 0.604 & 0.066 & 98 & 86 & 31 & 30 & -70 & 4.49E-7 & $1.99 \mathrm{E}-5$ & $6.28 \mathrm{E}-5$ \\
\hline MALME-3M & 0.598 & 1.040 & 1.014 & 0.949 & 0.784 & 0.788 & 0.374 & 94 & 79 & 42 & 43 & -37 & & $3.42 \mathrm{E}-5$ & $>1.00 \mathrm{E}-4$ \\
\hline & 0.642 & 2.291 & 2.207 & 2.088 & 0.941 & 1.157 & 0.67 & 95 & 88 & 18 & 31 & 2 & & $>1.00 \mathrm{E}-4$ & $>1.00 \mathrm{E}-4$ \\
\hline MDA-MB-435 & 0.697 & 2.816 & 2.671 & 1.733 & 0.580 & 0.673 & 0.47 & 93 & 49 & -17 & -4 & -33 & $9.43 \mathrm{E}-8$ & $5.54 \mathrm{E}-7$ & $>1.00 \mathrm{E}-4$ \\
\hline SK-MEL & 0.948 & 1.843 & 1.867 & 1.830 & 1.374 & 1.388 & 0.72 & 103 & 99 & 48 & 49 & -24 & $8.98 \mathrm{E}-7$ & $4.69 \mathrm{E}-5$ & $>1.00 \mathrm{E}-4$ \\
\hline SK-M & 0.770 & 1.958 & 1.961 & 1.801 & 1.381 & 1.469 & 0.7 & 100 & 87 & 51 & 59 & & 1.41 & $>1.00 \mathrm{E}-4$ & $>1.00 \mathrm{E}-4$ \\
\hline SK-M & 0.638 & 2.675 & 2.590 & 2.399 & 1.051 & 0.857 & 0.0 & 96 & 86 & 20 & 11 & -97 & & & $3.66 \mathrm{E}-5$ \\
\hline UACC & 0.623 & 1.725 & 1.642 & 1.559 & 1.183 & 1.180 & 0.520 & 92 & 85 & 51 & 51 & -17 & $1.02 \mathrm{E}-5$ & $5.67 \mathrm{E}-5$ & $>1.00 \mathrm{E}-4$ \\
\hline UACC-62 & 0.922 & 2.737 & 2.600 & 2.306 & 1.463 & 1.311 & 0.602 & 92 & 76 & 30 & 21 & -35 & 3.67E-7 & $2.41 \mathrm{E}-5$ & $>1.00 \mathrm{E}-4$ \\
\hline Ovarian Cancer & & & & & & & & & & & & & & & \\
\hline IGROV1 & 0.425 & 1.792 & 1.712 & 1.593 & 0.822 & 0.652 & 0.395 & 94 & 85 & 29 & 17 & -7 & $4.25 \mathrm{E}-7$ & $5.03 \mathrm{E}-5$ & $>1.00 \mathrm{E}-4$ \\
\hline OVCA & 0.77 & 2.104 & 2.155 & 2.085 & 1.016 & 0.743 & 0.381 & 104 & 99 & 18 & -4 & -51 & 4.04 & $2 \mathrm{E}-6$ & $9.72 \mathrm{E}-5$ \\
\hline & 0.7 & 1.670 & 1.631 & 1.560 & 1.258 & 1.003 & 0.725 & 96 & 89 & 57 & 31 & 2 & 1.89 & $>1.00 \mathrm{E}-4$ & $>1.00 \mathrm{E}-4$ \\
\hline & 0.4 & 1.298 & 1.240 & 1.215 & 0.934 & 0.735 & 0.5 & 93 & 90 & 55 & 30 & 4 & & $>1.0$ & $>1.00 \mathrm{E}-4$ \\
\hline OVCAI & 0.4 & 2.194 & 2.111 & 2.120 & 1.158 & 0.912 & 0.4 & 95 & 96 & 42 & 28 & 2 & 7.01E-7 & $>1.00 \mathrm{E}-4$ & $>1.00 \mathrm{E}-4$ \\
\hline NCI/ADR-RES & 0.420 & 1.420 & 1.381 & 1.280 & 0.593 & 0.402 & 0.330 & 96 & 86 & 17 & -4 & -21 & $3.34 \mathrm{E}-7$ & $6.27 \mathrm{E}-6$ & $>1.00 \mathrm{E}-4$ \\
\hline SK-OV-3 & 0.860 & 1.794 & 1.818 & 1.736 & 1.335 & 1.148 & 0.748 & 103 & 94 & 51 & 31 & -13 & $1.11 \mathrm{E}-6$ & $5.04 \mathrm{E}-5$ & $>1.00 \mathrm{E}-4$ \\
\hline Renal C & & & & & & & & & & & & & & & \\
\hline & 0.738 & 2.659 & 2.640 & 2.379 & 1.374 & 1.357 & 0.653 & 99 & 85 & 33 & 32 & -12 & & & $>1.00 \mathrm{E}-4$ \\
\hline & 1.682 & 2.297 & 2.241 & 2.135 & 1.620 & 1.532 & 1.05 & 91 & 74 & -4 & -9 & -37 & & & $1.00 \mathrm{E}-4$ \\
\hline & 0.300 & 1.195 & 1.198 & 1.147 & 0.652 & 0.582 & 0.22 & 100 & 95 & 39 & 31 & -2 & & & $>1.00 \mathrm{E}-4$ \\
\hline & 0.784 & 2.30 & 2.15 & 1.95 & 1.297 & 1.22 & 0.6 & 90 & 77 & 34 & 29 & -1 & & & $1.00 \mathrm{E}-4$ \\
\hline & 1.018 & 1.662 & 1.645 & 1.56 & 1.237 & $0.9 \mathrm{~s}$ & 0.73 & 97 & 85 & 34 & -2 & -2 & & & $1.00 \mathrm{E}-4$ \\
\hline SN12C & 1.2 & 3.2. & 3.164 & 3.1 & 2.198 & 1.83 & 0.9 & 97 & 95 & 48 & 30 & $-2 \quad-2 \quad-$ & & & $1.00 \mathrm{E}-4$ \\
\hline TK- & 0.872 & 1.776 & 1.679 & 1.612 & 1.389 & 1.169 & 0.7 & 89 & 82 & 57 & 33 & -13 & & & E-4 \\
\hline บO-31 & 0.903 & 1.912 & 1.712 & 1.659 & 1.344 & 1.112 & 0.742 & 80 & 75 & 44 & 21 & -18 & $6.27 \mathrm{E}-7$ & $3.45 \mathrm{E}-5$ & $>1.00 \mathrm{E}-4$ \\
\hline & & & & & & & & & & & & & & & \\
\hline & 0.550 & 1.893 & 1.823 & 1.657 & 1.142 & 0.908 & 0.572 & 95 & 82 & 44 & 27 & 2 & 7.00E-7 & $>1.00 \mathrm{E}-4$ & $>1.00 \mathrm{E}-4$ \\
\hline DU-145 & 0.429 & 1.801 & 1.823 & 1.750 & 1.151 & 0.716 & 0.482 & 102 & 96 & 53 & 21 & 4 & $1.21 \mathrm{E}-6$ & $>1.00 \mathrm{E}-4$ & $>1.00 \mathrm{E}-4$ \\
\hline Breast Can & & & & & & & & & & & & & & & \\
\hline MCF & 0.264 & 1.34 & 1.225 & 1.22 & 0.392 & 0.37 & & 89 & 89 & 12 & 10 & -1 & & & \\
\hline MD & 0.704 & 1.532 & 1.516 & 1.42 & 1.100 & 0.833 & 0.4 & 98 & 87 & 48 & 16 & & & & \\
\hline HS & 1.53 & 2.580 & 2.616 & 2.47 & 1.678 & $1.6 \mathrm{~s}$ & 1.1 & 103 & 90 & 14 & 15 & & & & \\
\hline & 1.118 & 2.405 & 2.386 & 2.244 & 1.574 & 1.518 & 0.685 & 99 & 87 & 35 & 31 & -39 & $5.24 \mathrm{E}-7$ & $2.79 \mathrm{E}-5$ & $1.00 \mathrm{E}-4$ \\
\hline T-47D & 0.832 & 1.990 & 1.933 & 1.808 & 1.176 & 1.066 & 0.803 & 95 & 84 & 30 & 20 & -4 & $4.24 \mathrm{E}-7$ & $7.09 \mathrm{E}-5$ & $1.00 \mathrm{E}-4$ \\
\hline
\end{tabular}


In-Vitro Testing Results

\begin{tabular}{|l|l|l|l|}
\hline NSC : D - 824738/1 & Experiment ID : 2010NS53 & Test Type : 08 & Units : Molar \\
\hline Report Date : March 24, 2021 & Test Date : October 05, 2020 & QNS : & MC : \\
\hline COMI : KLB2-25 & Stain Reagent : SRB Dual-Pass Related & SSPL : 1C1X & \\
\hline
\end{tabular}

Log10 Concentration

\begin{tabular}{|c|c|c|c|c|c|c|c|c|c|c|c|c|c|c|c|}
\hline \multirow[b]{2}{*}{ Panel/Cell Line } & \multirow{2}{*}{$\begin{array}{l}\text { Time } \\
\text { Zero }\end{array}$} & \multirow[b]{2}{*}{ Ctrl } & \multicolumn{5}{|c|}{ Mean Optical Densities } & \multicolumn{5}{|c|}{ ercent Growth } & \multirow[b]{2}{*}{ G150 } & \multirow[b]{2}{*}{ TGI } & \\
\hline & & & -8.0 & -7.0 & -6.0 & -5.0 & -4.0 & -8.0 & -7.0 & -6.0 & -5.0 & -4.0 & & & LC50 \\
\hline Leukemia & & & & & & & & & & & & & & & \\
\hline CCRF-CEM & 0.396 & 2.534 & 2.371 & 2.324 & 1.212 & 0.838 & 0.601 & 92 & 90 & 38 & 21 & 10 & $5.92 \mathrm{E}-7$ & $>1.00 \mathrm{E}-4$ & $>1.00 \mathrm{E}-4$ \\
\hline HL-60(TB) & 0.584 & 3.082 & 2.966 & 2.984 & 1.266 & 0.681 & 0.501 & 95 & 96 & 27 & 4 & -14 & $4.68 \mathrm{E}-7$ & 1.64E-5 & $>1.00 \mathrm{E}-4$ \\
\hline $\mathrm{K}-562$ & 0.151 & 1.869 & 1.861 & 1.738 & 0.528 & 0.346 & 0.225 & 100 & 92 & 22 & 11 & 4 & 4.00E-7 & $>1.00 \mathrm{E}-4$ & $>1.00 \mathrm{E}-4$ \\
\hline MOLT-4 & 0.495 & 2.947 & 2.882 & 2.967 & 1.906 & 1.041 & 0.672 & 97 & 101 & 58 & 22 & 7 & $1.64 \mathrm{E}-6$ & $>1.00 \mathrm{E}-4$ & $>1.00 \mathrm{E}-4$ \\
\hline RPMI-8226 & 0.802 & 2.818 & 2.854 & 2.761 & 2.136 & 1.297 & 0.849 & 102 & 97 & 66 & 25 & 2 & $2.44 \mathrm{E}-6$ & $>1.00 \mathrm{E}-4$ & $>1.00 \mathrm{E}-4$ \\
\hline & 0.285 & 1.337 & 1.342 & 1.291 & 0.644 & 0.573 & 0.395 & 100 & 96 & 34 & 27 & 10 & $5.51 \mathrm{E}-7$ & $>1.00 \mathrm{E}-4$ & $>1.00 \mathrm{E}-4$ \\
\hline Non-Small Cell Lung & Cancer & & & & & & & & & & & & & & \\
\hline A549/ATCC & 0.366 & 2.258 & 2.095 & 2.164 & 1.467 & 0.763 & 0.422 & 91 & 95 & 58 & 21 & 3 & $1.66 \mathrm{E}-6$ & $>1.00 \mathrm{E}-4$ & $>1.00 \mathrm{E}-4$ \\
\hline EKVX & 0.665 & 2.082 & 2.017 & 1.961 & 1.849 & 1.188 & 0.647 & 95 & 91 & 84 & 37 & -3 & $5.24 \mathrm{E}-6$ & 8.54E-5 & $>1.00 \mathrm{E}-4$ \\
\hline HOP-62 & 0.914 & 2.869 & 2.779 & 2.695 & 1.998 & 1.504 & 0.563 & 95 & 91 & 55 & 30 & -38 & $1.64 \mathrm{E}-6$ & $2.75 \mathrm{E}-5$ & $>1.00 \mathrm{E}-4$ \\
\hline HOP-92 & 1.314 & 1.878 & 1.757 & 1.693 & 1.624 & 1.256 & 0.576 & 79 & 67 & 55 & -4 & -56 & $1.21 \mathrm{E}-6$ & $8.42 \mathrm{E}-6$ & $7.60 \mathrm{E}-5$ \\
\hline $\mathrm{NCl}-\mathrm{H} 226$ & 0.717 & 1.549 & 1.535 & 1.536 & 1.433 & 0.978 & 0.559 & 98 & 98 & 86 & 31 & -22 & $4.55 \mathrm{E}-6$ & $3.86 \mathrm{E}-5$ & $>1.00 \mathrm{E}-4$ \\
\hline $\mathrm{NCl}-\mathrm{H} 23$ & 0.679 & 2.355 & 2.184 & 2.174 & 1.834 & 1.135 & 0.641 & 90 & 89 & 69 & 27 & -6 & $2.84 \mathrm{E}-6$ & $6.72 \mathrm{E}-5$ & $>1.00 \mathrm{E}-4$ \\
\hline $\mathrm{NCl}-\mathrm{H} 322 \mathrm{M}$ & 0.733 & 2.171 & 2.112 & 2.059 & 1.805 & 1.356 & 0.866 & 96 & 92 & 75 & 43 & 9 & $6.10 \mathrm{E}-6$ & $>1.00 \mathrm{E}-4$ & $>1.00 \mathrm{E}-4$ \\
\hline $\mathrm{NCl}-\mathrm{H} 460$ & 0.284 & 2.956 & 2.976 & 2.959 & 1.448 & 0.494 & 0.214 & 101 & 100 & 44 & 8 & -25 & 7.69E-7 & 1.74E-5 & $>1.00 \mathrm{E}-4$ \\
\hline $\mathrm{NCl}-\mathrm{H} 522$ & 1.353 & 3.173 & 3.138 & 3.064 & 2.405 & 1.821 & 1.115 & 98 & 94 & 58 & 26 & -18 & $1.75 \mathrm{E}-6$ & $3.92 \mathrm{E}-5$ & $>1.00 \mathrm{E}-4$ \\
\hline Colon Cancer & & & & & & & & & & & & & & & \\
\hline COLO 205 & 0.452 & $\begin{array}{l}1.831 \\
2003\end{array}$ & 1.752 & $\begin{array}{l}1.786 \\
\end{array}$ & $\begin{array}{l}1.346 \\
1.686\end{array}$ & 0.577 & 0.170 & 94 & 97 & 65 & 9 & -62 & 1.84E-6 & 1.34E-5 & $\begin{array}{r}6.71 \mathrm{E}-5 \\
-\end{array}$ \\
\hline HCT-116 & $\begin{array}{l}0.504 \\
0.311\end{array}$ & 2.967 & 2.949 & $\begin{array}{l}2.038 \\
2834\end{array}$ & $\begin{array}{l}1.686 \\
1014\end{array}$ & 0.882 & $\begin{array}{l}0.326 \\
0.071\end{array}$ & 94 & $\begin{array}{l}91 \\
95\end{array}$ & 74 & $\begin{array}{l}24 \\
17\end{array}$ & $\begin{array}{l}-30 \\
-77\end{array}$ & $\begin{array}{l}3.03 \mathrm{E}-6 \\
453 \mathrm{~F}-7\end{array}$ & $\begin{array}{l}2.52 \mathrm{E}-5 \\
150 \mathrm{~F}-5\end{array}$ & $\begin{array}{l}>1.00 \mathrm{E}-4 \\
512 \mathrm{~F}-5\end{array}$ \\
\hline HCT-15 & 0.272 & 2.071 & 2.007 & 1.979 & 0.949 & 0.641 & 0.277 & 96 & 95 & $\begin{array}{l}20 \\
38\end{array}$ & 21 & & $6.08 \mathrm{E}-7$ & $\begin{array}{l}1.50 E-5 \\
>1.00 E-4\end{array}$ & $>1.00 \mathrm{E}-4$ \\
\hline HT29 & 0.289 & 1.906 & 1.911 & 1.888 & 0.919 & 0.364 & 0.200 & 100 & 99 & 39 & 5 & -31 & $6.54 \mathrm{E}-7$ & 1.35 & $>1.00 \mathrm{E}-4$ \\
\hline KM12 & 0.372 & 2.164 & 2.254 & 2.118 & 1.115 & 0.767 & 0.242 & 105 & 97 & 41 & 22 & -35 & $7.03 \mathrm{E}-7$ & $2.44 \mathrm{E}-5$ & $>1.00 \mathrm{E}-4$ \\
\hline SW-620 & 0.269 & 2.198 & 2.187 & 2.211 & 1.138 & 0.652 & 0.405 & 99 & 101 & 45 & 20 & 7 & $8.14 \mathrm{E}-7$ & $>1.00 \mathrm{E}-4$ & $>1.00 \mathrm{E}-4$ \\
\hline CNS Cancer & & & & & & & & & & & & & & & \\
\hline SF-268 & 0.912 & 2.680 & 2.552 & 2.560 & 2.228 & 1.829 & 0.831 & 93 & 93 & 74 & 52 & -9 & 1.07E-5 & 7.13E-5 & $>1.00 \mathrm{E}-4$ \\
\hline SF-295 & 0.626 & 2.539 & 2.423 & 2.379 & 1.070 & 0.643 & 0.233 & 94 & 92 & 23 & 1 & -63 & 4.06E-7 & 1.03E-5 & $6.28 \mathrm{E}-5$ \\
\hline SF-539 & 0.703 & 2.378 & 2.363 & 2.272 & 1.626 & 0.713 & 0.321 & 99 & 94 & 55 & 1 & -54 & 1.24E-6 & $1.02 \mathrm{E}-5$ & $8.34 \mathrm{E}-5$ \\
\hline SNB-19 & 0.459 & 1.906 & 1.821 & 1.800 & 1.267 & 0.799 & 0.566 & 94 & 93 & 56 & 23 & 7 & $1.51 \mathrm{E}-6$ & $>1.00 \mathrm{E}-4$ & $>1.00 \mathrm{E}-4$ \\
\hline SNB-75 & 1.315 & 2.257 & 2.092 & 2.107 & 1.633 & 1.006 & 0.916 & 82 & 84 & 34 & -23 & -30 & $4.75 \mathrm{E}-7$ & $3.88 \mathrm{E}-6$ & $>1.00 \mathrm{E}-4$ \\
\hline U251 & 0.270 & 1.700 & 1.663 & 1.637 & 1.161 & 0.576 & 0.259 & 97 & 96 & 62 & 21 & -4 & $2.00 \mathrm{E}-6$ & $6.82 \mathrm{E}-5$ & $>1.00 \mathrm{E}-4$ \\
\hline Melanoma & & & & & & & & & & & & & & & \\
\hline LOX IM & 0.314 & 2.369 & 2.328 & 2.308 & 1.526 & 1.171 & 0.374 & 98 & 97 & 59 & 42 & 3 & 3.30E-6 & $>1.00 \mathrm{E}-4$ & $>1.00 \mathrm{E}-4$ \\
\hline MALME-3M & 0.623 & 1.472 & 1.454 & 1.432 & 1.077 & 1.066 & 0.521 & 98 & 95 & 53 & 52 & -16 & $1.08 \mathrm{E}-5$ & $5.76 \mathrm{E}-5$ & $>1.00 \mathrm{E}-4$ \\
\hline & 0.457 & 2.049 & 1.882 & 1.885 & 1.029 & 0.472 & 0.26 & 90 & 90 & 36 & 1 & -41 & & & $>1.00 \mathrm{E}-4$ \\
\hline MDA-MB-435 & 0.554 & 2.736 & 2.640 & 2.672 & 0.766 & 0.296 & 0.525 & 96 & 97 & 10 & -47 & -5 & $3.46 \mathrm{E}-7$ & & $>1.00 \mathrm{E}-4$ \\
\hline SK-MEL & 1.307 & 2.823 & 2.862 & 2.758 & 2.275 & 2.109 & 1.173 & 103 & 96 & 64 & 53 & -10 & $1.11 \mathrm{E}-5$ & $6.88 \mathrm{E}-5$ & $>1.00 \mathrm{E}-4$ \\
\hline SK-M & 0.602 & 1.992 & 1.998 & 1.925 & 1.382 & 1.275 & 0.576 & 100 & 95 & 56 & 48 & -4 & $6.21 \mathrm{E}-6$ & & $>1.00 \mathrm{E}-4$ \\
\hline SK-M & 1.055 & 3.325 & 3.341 & 3.321 & 2.630 & 1.793 & 0.383 & 101 & 100 & 69 & 33 & -64 & $3.36 \mathrm{E}-6$ & & $7.21 \mathrm{E}-5$ \\
\hline UACC & 1.286 & 2.955 & 2.819 & 2.759 & 2.476 & 2.376 & 1.008 & 92 & 88 & 71 & 65 & -22 & $1.50 \mathrm{E}-5$ & $5.64 \mathrm{E}-5$ & $>1.00 \mathrm{E}-4$ \\
\hline UACC-62 & 0.616 & 2.467 & 2.301 & 2.236 & 1.081 & 0.911 & 0.456 & 91 & 88 & 25 & 16 & -26 & $3.99 \mathrm{E}-7$ & $2.39 E-5$ & $>1.00 \mathrm{E}-4$ \\
\hline Ovarian Cancer & & & & & & & & & & & & & & & \\
\hline IGROV1 & 0.341 & 1.739 & 1.694 & 1.640 & 0.916 & 0.643 & 0.368 & 97 & 93 & 41 & 22 & 2 & $6.73 \mathrm{E}-7$ & $>1.00 \mathrm{E}-4$ & $>1.00 \mathrm{E}-4$ \\
\hline & 0.5 & 1.982 & 2.022 & 2.039 & 0.934 & 0.642 & 0.328 & 103 & 104 & 28 & 8 & -38 & $5.12 \mathrm{E}-7$ & & $>1.00 \mathrm{E}-4$ \\
\hline & 0.9 & 1.858 & 1.914 & 1.864 & 1.711 & 1.419 & 0.958 & 106 & 101 & 84 & 53 & 5 & 1.18E-5 & $>1.0$ & $>1.00 \mathrm{E}-4$ \\
\hline OVCAF & 0.52 & 1.585 & 1.510 & 1.536 & 1.342 & 0.805 & 0.635 & 93 & 95 & 77 & 26 & 10 & & & $>1.00 \mathrm{E}-4$ \\
\hline OVCAF & 0.43 & 2.397 & 2.370 & 2.392 & 2.026 & 1.008 & 0.456 & 99 & 100 & 81 & 29 & 1 & & $>1.00 \mathrm{E}-4$ & $>1.00 \mathrm{E}-4$ \\
\hline NCI/ADR-RES & 0.374 & 1.536 & 1.478 & 1.569 & 0.984 & 0.649 & 0.366 & 95 & 103 & 53 & 24 & -2 & & $8.26 \mathrm{E}-5$ & $>1.00 \mathrm{E}-4$ \\
\hline SK-OV-3 & 0.781 & 1.882 & 1.847 & 1.801 & 1.610 & 1.225 & 0.758 & 97 & 93 & 75 & 40 & -3 & $5.30 \mathrm{E}-6$ & $8.55 \mathrm{E}-5$ & $>1.00 \mathrm{E}-4$ \\
\hline Renal C & & & & & & & & & & & & & & & \\
\hline & 0.675 & 2.617 & 2.474 & 2.348 & 1.389 & 1.341 & 0.18 & 93 & 86 & 37 & 34 & -73 & & & $6.12 \mathrm{E}-5$ \\
\hline & 0.453 & 2.008 & 2.016 & 2.029 & 1.546 & 1.031 & 0.4 & 100 & 101 & 70 & 37 & & & & \\
\hline & 0.782 & 2.47 & 2.34 & $2.2 \varepsilon$ & 1.475 & $1.2 \varepsilon$ & $0.6 s$ & 93 & 89 & 41 & 30 & -12 & & & - $1.00 \mathrm{E}-4$ \\
\hline & 0.9 & 1.64 & 1.61 & 1.617 & 1.329 & $0.8 \varepsilon$ & 0.6 & 94 & 95 & 51 & -11 & -32 & & & $E-4$ \\
\hline SN12C & 0.46 & 1.87 & 1.79 & $1.7 \mathrm{~s}$ & 1.552 & 0.95 & 0.46 & 94 & 94 & 77 & 35 & & & 1.0 & \\
\hline TK-1 & 852 & 1.953 & 1.856 & 1.788 & 1.793 & 1.486 & 0.8 & 91 & 85 & 85 & 58 & & & & $1.00 \mathrm{E}-4$ \\
\hline บO-31 & 0.759 & 2.508 & 2.354 & 2.356 & 1.840 & 1.321 & 0.621 & 91 & 91 & 62 & 32 & -18 & $2.49 \mathrm{E}-6$ & $4.35 \mathrm{E}-5$ & $>1.00 \mathrm{E}-4$ \\
\hline Prostate Cancer & & & & & & & & & & & & & & & \\
\hline & 0.503 & 2.185 & 1.967 & 1.895 & 1.161 & 0.781 & 0.227 & 87 & 83 & 39 & 17 & -55 & 5.63E-7 & 1.70E-5 & 8.55E-5 \\
\hline DU-145 & 0.394 & 1.887 & 1.949 & 1.891 & 1.711 & 0.712 & 0.582 & 104 & 100 & 88 & 21 & 13 & $3.72 \mathrm{E}-6$ & $>1.00 \mathrm{E}-4$ & $>1.00 \mathrm{E}-4$ \\
\hline Breast $\mathrm{Ca}$ & & & & & & & & & & & & & & & \\
\hline & 0.400 & 2.245 & 2.091 & 2.193 & 1.026 & 0.672 & 0.42 & 92 & & 34 & 15 & & & & $1.00 E-4$ \\
\hline MDA-MB-2 & 0.569 & 1.375 & 1.359 & 1.380 & 1.239 & 0.819 & 0.467 & 98 & 101 & 83 & 31 & -18 & $4.31 \mathrm{E}-6$ & 4.30E-5 & $1.00 \mathrm{E}-4$ \\
\hline HS 57 & 1.541 & 2.376 & 2.458 & 2.357 & 1.886 & 1.624 & 1.292 & 110 & 98 & 41 & 10 & -16 & 7.01 & & $1.00 \mathrm{E}-4$ \\
\hline BT-54 & 1.345 & 2.461 & 2.471 & 2.324 & 1.679 & 1.503 & 0.43 & 101 & 88 & 30 & 14 & -68 & & & $6.09 \mathrm{E}-5$ \\
\hline T-47D & 0.705 & 1.770 & 1.714 & 1.613 & 1.303 & 1.075 & 0.797 & 95 & 85 & 56 & 35 & & 1.94E-6 & 1.00E-4 & $.00 \mathrm{E}-4$ \\
\hline MDA-MB-468 & 0.760 & 1.756 & 1.630 & 1.697 & 1.217 & 0.916 & 0.631 & 87 & 94 & 46 & 16 & -17 & $8.21 \mathrm{E}-7$ & $3.01 \mathrm{E}-5$ & 1.00E-4 \\
\hline
\end{tabular}


In-Vitro Testing Results

\begin{tabular}{|l|l|l|l|}
\hline NSC : D - 824739/1 & Experiment ID : 2010NS53 & Test Type : 08 & Units : Molar \\
\hline Report Date : March 24, 2021 & Test Date : October 05, 2020 & QNS : & MC : \\
\hline COMI : KLB2-26 & Stain Reagent : SRB Dual-Pass Related & SSPL : 1C1X & \\
\hline
\end{tabular}

Log10 Concentration

\begin{tabular}{|c|c|c|c|c|c|c|c|c|c|c|c|c|c|c|c|}
\hline \multirow[b]{2}{*}{ Panel/Cell Line } & \multirow{2}{*}{$\begin{array}{l}\text { Time } \\
\text { Zero }\end{array}$} & \multirow[b]{2}{*}{ Ctrl } & \multicolumn{5}{|c|}{ Mean Optical Densities } & \multicolumn{5}{|c|}{ Percent Growth } & \multirow[b]{2}{*}{ GI50 } & & \\
\hline & & & -8.0 & -7.0 & -6.0 & -5.0 & -4.0 & -8.0 & -7.0 & -6.0 & -5.0 & -4.0 & & TGI & LC50 \\
\hline Leukemia & & & & & & & & & & & & & & & \\
\hline CCRF-CEM & 0.396 & 2.525 & 2.522 & 2.425 & 1.895 & 0.771 & 0.703 & 100 & 95 & 70 & 18 & 14 & $2.43 \mathrm{E}-6$ & $>1.00 \mathrm{E}-4$ & $>1.00 \mathrm{E}-4$ \\
\hline HL-60(TB) & 0.584 & 2.828 & 2.768 & 2.885 & 2.208 & 0.676 & 0.437 & 97 & 103 & 72 & 4 & -25 & $2.12 \mathrm{E}-6$ & 1.38E-5 & $>1.00 \mathrm{E}-4$ \\
\hline $\mathrm{K}-562$ & 0.151 & 1.682 & 1.715 & 1.690 & 0.679 & 0.409 & 0.298 & 102 & 101 & 34 & 17 & 10 & $5.82 \mathrm{E}-7$ & $>1.00 \mathrm{E}-4$ & $>1.00 \mathrm{E}-4$ \\
\hline MOLT-4 & 0.495 & 2.800 & 2.811 & 2.806 & 2.359 & 1.116 & 0.674 & 100 & 100 & 81 & 27 & 8 & 3.73E-6 & $>1.00 \mathrm{E}-4$ & $>1.00 \mathrm{E}-4$ \\
\hline RPMI-8226 & 0.802 & 2.813 & 2.833 & 2.833 & 2.505 & 1.205 & 0.767 & 101 & 101 & 85 & 20 & -4 & 3.44E-6 & 6.62E-5 & $>1.00 \mathrm{E}-4$ \\
\hline & 0.285 & 1.303 & 1.299 & 1.263 & 0.603 & 0.526 & 0.399 & 100 & 96 & 31 & 24 & 11 & $5.13 \mathrm{E}-7$ & $>1.00 \mathrm{E}-4$ & $>1.00 \mathrm{E}-4$ \\
\hline Non-Small Cell Lung & Cancer & & & & & & & & & & & & & & \\
\hline A549/ATCC & 0.366 & 2.227 & 2.116 & 2.180 & 1.671 & 1.026 & 0.429 & 94 & 97 & 70 & 35 & 3 & $3.80 \mathrm{E}-6$ & $>1.00 \mathrm{E}-4$ & $>1.00 \mathrm{E}-4$ \\
\hline EKVX & 0.665 & 2.174 & 2.143 & 2.130 & 1.959 & 1.215 & 0.724 & 98 & 97 & 86 & 36 & 4 & $5.31 \mathrm{E}-6$ & $>1.00 \mathrm{E}-4$ & $>1.00 \mathrm{E}-4$ \\
\hline HOP-62 & 0.914 & 2.748 & 2.748 & 2.717 & 2.176 & 1.346 & 0.721 & 100 & 98 & 69 & 24 & -21 & $2.60 \mathrm{E}-6$ & 3.36E-5 & $>1.00 \mathrm{E}-4$ \\
\hline HOP-92 & 1.314 & 1.864 & 1.775 & 1.813 & 1.666 & 1.323 & 0.953 & 84 & 91 & 64 & 2 & -28 & $1.68 \mathrm{E}-6$ & $1.14 \mathrm{E}-5$ & $>1.00 \mathrm{E}-4$ \\
\hline $\mathrm{NCl}-\mathrm{H} 226$ & 0.717 & 1.616 & 1.561 & 1.551 & 1.424 & 0.987 & 0.635 & 94 & 93 & 79 & 30 & -11 & $3.88 \mathrm{E}-6$ & $5.30 \mathrm{E}-5$ & $>1.00 \mathrm{E}-4$ \\
\hline $\mathrm{NCl}-\mathrm{H} 23$ & 0.679 & 2.317 & 2.227 & 2.319 & 2.016 & 1.229 & 0.650 & 95 & 100 & 82 & 34 & -4 & 4.55E-6 & 7.71E-5 & $>1.00 \mathrm{E}-4$ \\
\hline $\mathrm{NCl}-\mathrm{H} 322 \mathrm{M}$ & 0.733 & 2.199 & 2.111 & 2.206 & 2.061 & 1.306 & 0.922 & 94 & 100 & 91 & 39 & 13 & $6.14 \mathrm{E}-6$ & $>1.00 \mathrm{E}-4$ & $>1.00 \mathrm{E}-4$ \\
\hline $\mathrm{NCl}-\mathrm{H} 460$ & 0.284 & 2.887 & 2.982 & 2.989 & 2.334 & 0.556 & 0.224 & 104 & 104 & 79 & 10 & -21 & 2.64E-6 & 2.14E-5 & $>1.00 \mathrm{E}-4$ \\
\hline $\mathrm{NCl}-\mathrm{H} 522$ & 1.353 & 3.150 & 3.042 & 3.024 & 2.448 & 1.810 & 1.130 & 94 & 93 & 61 & 25 & -17 & $2.03 \mathrm{E}-6$ & $4.04 \mathrm{E}-5$ & $>1.00 \mathrm{E}-4$ \\
\hline Colon Cancer & & & & & & & & & & & & & & & \\
\hline COLO 205 & 0.452 & 1.664 & 1.691 & 1.625 & 1.304 & 0.530 & 0.161 & 102 & 97 & 70 & 6 & -64 & $2.08 \mathrm{E}-6$ & 1.23E-5 & $6.26 \mathrm{E}-5$ \\
\hline HCC-2998 & 0.504 & 2.043 & 1.895 & 2.004 & 1.711 & 1.127 & 0.392 & 90 & 97 & 78 & 40 & -22 & $5.62 \mathrm{E}-6$ & $4.42 \mathrm{E}-5$ & $>1.00 \mathrm{E}-4$ \\
\hline HCT-116 & 0.311 & 2.937 & 2.845 & 2.873 & 2.269 & 0.932 & 0.394 & 96 & 98 & 75 & 24 & 3 & 3.04E-6 & $>1.00 \mathrm{E}-4$ & $>1.00 \mathrm{E}-4$ \\
\hline HCT-15 & 0.272 & 2.063 & 2.007 & 1.949 & 1.026 & 0.608 & 0.248 & 97 & 94 & 42 & 19 & -9 & 7.02E-7 & $4.79 \mathrm{E}-5$ & $>1.00 \mathrm{E}-4$ \\
\hline HT29 & 0.289 & 1.852 & 1.847 & 1.852 & 1.309 & 0.336 & 0.220 & 100 & 100 & 65 & 3 & -24 & $1.76 \mathrm{E}-6$ & $1.29 \mathrm{E}-5$ & $>1.00 \mathrm{E}-4$ \\
\hline KM12 & 0.372 & 2.278 & 2.265 & 2.164 & 1.271 & 0.778 & 0.295 & 99 & 94 & 47 & 21 & -21 & $8.69 \mathrm{E}-7$ & $3.20 \mathrm{E}-5$ & $>1.00 \mathrm{E}-4$ \\
\hline SW-620 & 0.269 & 2.259 & 2.171 & 2.148 & 1.303 & 0.535 & 0.369 & 96 & 94 & 52 & 13 & 5 & $1.12 \mathrm{E}-6$ & $>1.00 \mathrm{E}-4$ & $>1.00 \mathrm{E}-4$ \\
\hline CNS Cancer & & & & & & & & & & & & & & & \\
\hline SF-268 & 0.912 & 2.683 & 2.573 & 2.647 & 2.431 & 1.666 & 0.736 & 94 & 98 & 86 & 43 & -19 & $6.73 \mathrm{E}-6$ & $4.88 \mathrm{E}-5$ & $>1.00 \mathrm{E}-4$ \\
\hline SF-295 & 0.626 & 2.611 & 2.396 & 2.537 & 1.734 & 0.688 & 0.354 & 89 & 96 & 56 & $\begin{array}{l}3 \\
0\end{array}$ & -43 & 1.29E-6 & 1.17E-5 & $>1.00 \mathrm{E}-4$ \\
\hline SF-539 & 0.703 & 2.422 & 2.408 & 2.419 & 2.042 & 0.847 & 0.401 & 99 & 100 & 78 & 8 & -43 & $2.52 \mathrm{E}-6$ & 1.46E-5 & $>1.00 \mathrm{E}-4$ \\
\hline SNB-19 & 0.459 & 1.882 & 1.815 & 1.868 & 1.554 & 0.884 & 0.584 & 95 & 99 & 77 & 30 & 9 & $3.73 \mathrm{E}-6$ & $>1.00 \mathrm{E}-4$ & $>1.00 \mathrm{E}-4$ \\
\hline SNB-75 & 1.315 & 2.244 & 1.974 & 2.018 & 1.795 & 0.863 & 0.631 & 71 & 76 & 52 & -34 & -52 & $1.05 \mathrm{E}-6$ & $3.98 \mathrm{E}-6$ & $7.68 \mathrm{E}-5$ \\
\hline U251 & 0.270 & 1.677 & 1.636 & 1.641 & 1.409 & 0.565 & 0.301 & 97 & 97 & 81 & 21 & 2 & $3.28 \mathrm{E}-6$ & $>1.00 \mathrm{E}-4$ & $>1.00 \mathrm{E}-4$ \\
\hline Melanoma & & & & & & & & & & & & & & & \\
\hline LOX IM & 0.314 & 2.378 & 2.281 & 2.354 & 1.937 & 1.010 & 0.318 & 95 & 99 & 79 & 34 & & $4.34 \mathrm{E}-6$ & $>1.00 \mathrm{E}-4$ & $>1.00 \mathrm{E}-4$ \\
\hline MALME-3M & 0.623 & 1.495 & 1.472 & 1.474 & 1.157 & 0.950 & 0.581 & 97 & 98 & 61 & 37 & -7 & $2.98 \mathrm{E}-6$ & $7.01 \mathrm{E}-5$ & $>1.00 \mathrm{E}-4$ \\
\hline & 0.457 & 2.125 & 2.056 & 2.077 & 1.497 & 0.723 & 0.53 & 96 & 97 & 62 & 16 & 4 & & & $>1.00 \mathrm{E}-4$ \\
\hline MDA-MB-435 & 0.554 & 2.632 & 2.524 & 2.446 & 0.684 & 0.481 & 0.393 & 95 & 91 & 6 & -13 & -29 & 3.05 & $2.09 \mathrm{E}-6$ & $>1.00 \mathrm{E}-4$ \\
\hline SK-ME & 1.307 & 2.762 & 2.767 & 2.756 & 2.402 & 1.841 & 1.012 & 100 & 100 & 75 & 37 & -23 & 4.51 & & $>1.00 \mathrm{E}-4$ \\
\hline SK-M & 0.602 & 1.999 & 1.996 & 2.013 & 1.589 & 1.371 & 0.80 & 100 & 101 & 71 & 55 & 15 & & $>1.00 \mathrm{E}-4$ & $>1.00 \mathrm{E}-4$ \\
\hline SK-M & 1.055 & 3.321 & 3.327 & 3.339 & 3.093 & 1.334 & 0.07 & 100 & 101 & 90 & 12 & -93 & & & $3.90 \mathrm{E}-5$ \\
\hline UACC & 1.286 & 2.880 & 2.820 & 2.840 & 2.611 & 2.197 & 1.006 & 96 & 98 & 83 & 57 & -22 & $1.23 \mathrm{E}-5$ & $5.29 \mathrm{E}-5$ & $>1.00 \mathrm{E}-4$ \\
\hline UACC-62 & 0.616 & 2.469 & 2.322 & 2.319 & 1.521 & 0.943 & 0.364 & 92 & 92 & 49 & 18 & -41 & $9.39 \mathrm{E}-7$ & $2.00 E-5$ & $>1.00 \mathrm{E}-4$ \\
\hline Ovarian Cancer & & & & & & & & & & & & & & & \\
\hline IGROV1 & 0.341 & 1.740 & 1.726 & 1.803 & 1.212 & 0.647 & 0.385 & 99 & 105 & 62 & 22 & 3 & $2.01 \mathrm{E}-6$ & $>1.00 \mathrm{E}-4$ & $>1.00 \mathrm{E}-4$ \\
\hline & & 1.957 & 1.913 & 1.974 & 1.393 & 0.625 & 0.3 & 97 & 101 & 60 & 7 & -28 & 1.57E-6 & $E-5$ & $>1.00 \mathrm{E}-4$ \\
\hline & & 1.809 & 1.787 & 1.837 & 1.721 & 1.256 & 0.8 & 98 & 103 & 90 & 38 & -2 & $5.93 \mathrm{E}-6$ & & $>1.00 \mathrm{E}-4$ \\
\hline & & 1.597 & 1.579 & 1.612 & 1.471 & 0.95 & 0.5 & 98 & 101 & 88 & 40 & 4 & & $>1.0$ & $>1.00 \mathrm{E}-4$ \\
\hline & 0.43 & 2.386 & 2.392 & 2.411 & 2.103 & 1.015 & 0.5 & 100 & 101 & 85 & 30 & 4 & & $>1.00 \mathrm{E}-4$ & $>1.00 \mathrm{E}-4$ \\
\hline NCI/ADR-RES & 0.374 & 1.498 & 1.464 & 1.500 & 1.034 & 0.607 & 0.359 & 97 & 100 & 59 & 21 & -4 & & 6.81E-5 & $>1.00 \mathrm{E}-4$ \\
\hline SK-OV-3 & 0.781 & 1.773 & 1.800 & 1.813 & 1.576 & 1.061 & 0.648 & 103 & 104 & 80 & 28 & -17 & $3.80 \mathrm{E}-6$ & 4.20E-5 & $>1.00 \mathrm{E}-4$ \\
\hline Renal Ca & & & & & & & & & & & & & & & \\
\hline & 0.675 & 2.641 & 2.625 & 2.665 & 2.495 & 1.305 & 0.70 & 99 & 101 & 93 & 32 & 1 & & & $1.00 \mathrm{E}-4$ \\
\hline & 453 & 2.027 & 2.047 & 2.079 & 1.843 & 0.972 & 0.4 & 101 & 103 & 88 & 33 & & & 1. & \\
\hline & 0.782 & 2.35 & 2.24 & 2.15 & 1.599 & 1.15 & 0.6 & 93 & 87 & 52 & 24 & -17 & & & $>1.0$ \\
\hline & 0 & 1.68 & 1.64 & 1.67 & 1.461 & 0.8 & 0.6 & 94 & 99 & 68 & -14 & -34 & & & $>1.0$ \\
\hline SN12C & 0.460 & 1.79 & 1.81 & $1.8 \varepsilon$ & 1.742 & 0.8 & 0.42 & 102 & 107 & 96 & 29 & -8 & & & $E-4$ \\
\hline TK-1 & 852 & 1.862 & 1.778 & 1.799 & 1.818 & 1.326 & 0.7 & 92 & 94 & 96 & 47 & -9 & & & $>1.00 \mathrm{E}-4$ \\
\hline บO-31 & 0.759 & 2.506 & 2.381 & 2.416 & 2.179 & 1.366 & 0.790 & 93 & 95 & 81 & 35 & 2 & $4.69 \mathrm{E}-6$ & $>1.00 \mathrm{E}-4$ & $>1.00 \mathrm{E}-4$ \\
\hline Prostate Cancer & & & & & & & & & & & & & & & \\
\hline & 0.503 & 2.109 & 2.122 & 2.034 & 1.528 & 0.786 & 0.566 & 101 & 95 & 64 & 18 & 4 & & $>1.00 \mathrm{E}-4$ & $>1.00 \mathrm{E}-4$ \\
\hline DU-145 & 0.394 & 1.903 & 1.910 & 1.952 & 1.768 & 0.760 & 0.447 & 100 & 103 & 91 & 24 & 3 & 4.11E-6 & $>1.00 \mathrm{E}-4$ & $>1.00 \mathrm{E}-4$ \\
\hline Breast $\mathrm{Ca}$ & & & & & & & & & & & & & & & \\
\hline MCF & 0.400 & 2.293 & 2.158 & 2.241 & 1.483 & 0.643 & & 93 & & 57 & 13 & & & & \\
\hline MDA-N & 0.569 & 1.450 & 1.465 & 1.462 & 1.365 & 0.926 & 0.428 & 102 & 101 & 90 & 41 & -25 & $6.46 \mathrm{E}$ & & $>1.00 \mathrm{E}-4$ \\
\hline HS 57 & 1.541 & 2.469 & 2.431 & 2.439 & 2.187 & 1.466 & 1.047 & 96 & 97 & 70 & -5 & -32 & 1.83 & & > $1.00 \mathrm{E}-4$ \\
\hline BT-54 & 1.345 & 2.588 & 2.404 & 2.578 & 2.248 & 1.709 & 1.15 & 85 & 99 & 73 & 29 & -14 & & & $E-4$ \\
\hline T-47! & 0.705 & 1.639 & 1.561 & 1.634 & 1.496 & 0.834 & 0.695 & 92 & 99 & 85 & 14 & -1 & $3.08 \mathrm{E}-6$ & $7.99 \mathrm{E}-5$ & $.00 \mathrm{E}-4$ \\
\hline MDA-MB-468 & 0.760 & 1.717 & 1.731 & 1.605 & 1.305 & 0.821 & 0.617 & 101 & 88 & 57 & 6 & -19 & $1.37 \mathrm{E}-6$ & $1.79 \mathrm{E}-5$ & $>1.00 \mathrm{E}-4$ \\
\hline
\end{tabular}


In-Vitro Testing Results

\begin{tabular}{|l|l|l|l|}
\hline NSC : D - 824740 / 1 & Experiment ID : 2010NS53 & Test Type : 08 & Units : Molar \\
\hline Report Date : March 24, 2021 & Test Date : October 05, 2020 & QNS : & MC: \\
\hline COMI : KLB2-28 & Stain Reagent : SRB Dual-Pass Related & SSPL : 1C1X & \\
\hline
\end{tabular}

Log10 Concentration

\begin{tabular}{|c|c|c|c|c|c|c|c|c|c|c|c|c|c|c|c|}
\hline \multirow[b]{2}{*}{ Panel/Cell Line } & \multirow{2}{*}{$\begin{array}{l}\text { Time } \\
\text { Zero }\end{array}$} & \multirow[b]{2}{*}{ Ctrl } & \multicolumn{5}{|c|}{ Mean Optical Densities } & \multicolumn{5}{|c|}{ Percent Growth } & \multirow[b]{2}{*}{ G150 } & \multirow[b]{2}{*}{ TGI } & \\
\hline & & & -8.0 & -7.0 & -6.0 & -5.0 & -4.0 & -8.0 & -7.0 & -6.0 & -5.0 & -4.0 & & & LC50 \\
\hline Leukemia & & & & & & & & & & & & & & & \\
\hline CCRF-CEM & 0.396 & 2.530 & 2.446 & 2.238 & 0.872 & 0.804 & 0.599 & 96 & 86 & 22 & 19 & 10 & 3.69E-7 & $>1.00 \mathrm{E}-4$ & $>1.00 \mathrm{E}-4$ \\
\hline HL-60(TB) & 0.584 & 3.011 & 2.924 & 2.779 & 0.728 & 0.551 & 0.384 & 96 & 90 & 6 & -6 & -34 & 3.01E-7 & 3.25E-6 & $>1.00 \mathrm{E}-4$ \\
\hline $\mathrm{K}-562$ & 0.151 & 1.794 & 1.664 & 0.913 & 0.366 & 0.305 & 0.190 & 92 & 46 & 13 & 9 & 2 & $8.34 \mathrm{E}-8$ & $>1.00 \mathrm{E}-4$ & $>1.00 \mathrm{E}-4$ \\
\hline MOLT-4 & 0.495 & 2.801 & 2.831 & 2.793 & 1.275 & 0.929 & 0.594 & 101 & 100 & 34 & 19 & 4 & $5.68 \mathrm{E}-7$ & $>1.00 \mathrm{E}-4$ & $>1.00 \mathrm{E}-4$ \\
\hline RPMI-8226 & 0.802 & 2.815 & 2.797 & 2.702 & 1.523 & 1.320 & 0.718 & 99 & 94 & 36 & 26 & -11 & $5.72 \mathrm{E}-7$ & 5.12E-5 & $>1.00 \mathrm{E}-4$ \\
\hline & 0.285 & 1.294 & 1.164 & 0.735 & 0.576 & 0.515 & 0.320 & 87 & 45 & 29 & 23 & 3 & 7.44E-8 & $>1.00 \mathrm{E}-4$ & $>1.00 \mathrm{E}-4$ \\
\hline Non-Small Cell Lung & Cancer & & & & & & & & & & & & & & \\
\hline A549/ATCC & 0.366 & 2.134 & 2.063 & 2.002 & 1.196 & 0.818 & 0.391 & 96 & 93 & 47 & 26 & 1 & $8.57 \mathrm{E}-7$ & $>1.00 \mathrm{E}-4$ & $>1.00 \mathrm{E}-4$ \\
\hline EKVX & 0.665 & 2.116 & 2.026 & 1.981 & 1.465 & 1.129 & 0.662 & 94 & 91 & 55 & 32 & & $1.66 \mathrm{E}-6$ & 9.63E-5 & $>1.00 \mathrm{E}-4$ \\
\hline HOP-62 & 0.914 & 2.847 & 2.741 & 2.578 & 1.565 & 1.392 & 0.214 & 94 & 86 & 34 & 25 & -77 & $4.88 \mathrm{E}-7$ & $1.75 \mathrm{E}-5$ & $5.46 \mathrm{E}-5$ \\
\hline HOP-92 & 1.314 & 1.831 & 1.794 & 1.705 & 1.563 & 1.389 & 0.256 & 93 & 75 & 48 & 14 & -81 & $8.55 \mathrm{E}-7$ & $1.42 \mathrm{E}-5$ & 4.77E-5 \\
\hline $\mathrm{NCl}-\mathrm{H} 226$ & 0.717 & 1.564 & 1.452 & 1.457 & 1.114 & 0.893 & 0.224 & 87 & 87 & 47 & 21 & -69 & $8.35 \mathrm{E}-7$ & $1.70 \mathrm{E}-5$ & $6.17 \mathrm{E}-5$ \\
\hline $\mathrm{NCl}-\mathrm{H} 23$ & 0.679 & 2.282 & 2.117 & 2.060 & 1.416 & 0.959 & 0.413 & 90 & 86 & 46 & 17 & -39 & 7.92E-7 & 2.03E-5 & $>1.00 \mathrm{E}-4$ \\
\hline $\mathrm{NCl}-\mathrm{H} 322 \mathrm{M}$ & 0.733 & 2.151 & 2.084 & 2.044 & 1.500 & 1.326 & 0.525 & 95 & 92 & 54 & 42 & -28 & $2.15 \mathrm{E}-6$ & $3.94 \mathrm{E}-5$ & $>1.00 \mathrm{E}-4$ \\
\hline $\mathrm{NCl}-\mathrm{H} 460$ & 0.284 & 2.902 & 2.775 & 2.904 & 0.784 & 0.440 & 0.134 & 95 & 100 & 19 & 6 & -53 & $4.15 \mathrm{E}-7$ & $1.26 \mathrm{E}-5$ & $8.90 \mathrm{E}-5$ \\
\hline $\mathrm{NCl}-\mathrm{H} 522$ & 1.353 & 3.141 & 3.057 & 2.882 & 2.079 & 1.737 & 0.891 & 95 & 86 & 41 & 21 & -34 & $6.18 \mathrm{E}-7$ & $2.43 \mathrm{E}-5$ & $>1.00 \mathrm{E}-4$ \\
\hline Colon Cancer & & & & & & & & & & & & & & & \\
\hline COLO 205 & 0.452 & $\begin{array}{l}1.761 \\
1000\end{array}$ & 1.708 & $\begin{array}{l}1.711 \\
1882\end{array}$ & 0.953 & 0.629 & 0.199 & 96 & 96 & 38 & 14 & -56 & 6.28E-7 & 1.56E-5 & $8.18 \mathrm{E}-5$ \\
\hline HCT-116 & 0.311 & $\begin{array}{l}1.890 \\
2.838\end{array}$ & $\begin{array}{l}.1040 \\
2.829\end{array}$ & 2556 & 0.895 & 0.080 & $\begin{array}{l}0.104 \\
0.100\end{array}$ & 100 & $\begin{array}{l}50 \\
89\end{array}$ & $\begin{array}{l}54 \\
23\end{array}$ & 15 & $\begin{array}{l}-19 \\
-68\end{array}$ & $\begin{array}{l}1.25 \mathrm{E}-6 \\
390 \mathrm{E}-7\end{array}$ & $\begin{array}{l}1.3 / \mathrm{E}-5 \\
150 \mathrm{E}-5\end{array}$ & 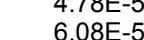 \\
\hline HCT-15 & 0.272 & 1.985 & 1.911 & 1.412 & 0.624 & 0.505 & 0.120 & 96 & 67 & 21 & 14 & -56 & $2.29 \mathrm{E}-7$ & $1.57 \mathrm{E}-5$ & $8.18 \mathrm{E}-5$ \\
\hline HT29 & 0.289 & 1.826 & 1.800 & 1.742 & 0.383 & 0.343 & 0.107 & 98 & 95 & 6 & 4 & -63 & $3.19 \mathrm{E}-7$ & $1.13 \mathrm{E}-5$ & $6.38 \mathrm{E}-5$ \\
\hline KM12 & 0.372 & 2.134 & 2.107 & 1.667 & 0.775 & 0.609 & 0.156 & 98 & 73 & 23 & 13 & -58 & $2.91 \mathrm{E}-7$ & $1.54 \mathrm{E}-5$ & $7.71 \mathrm{E}-5$ \\
\hline SW-620 & 0.269 & 2.238 & 2.088 & 2.026 & 0.737 & 0.661 & 0.275 & 92 & 89 & 24 & 20 & & $3.98 \mathrm{E}-7$ & $>1.00 \mathrm{E}-4$ & $>1.00 \mathrm{E}-4$ \\
\hline CNS Cancer & & & & & & & & & & & & & & & \\
\hline SF-268 & 0.912 & 2.541 & 2.468 & 2.372 & 1.683 & 1.701 & 0.322 & 96 & 90 & 47 & 48 & -65 & 8.65E-7 & $2.68 \mathrm{E}-5$ & 7.42E-5 \\
\hline SF-295 & 0.626 & 2.543 & 2.428 & 2.201 & 0.723 & 0.594 & 0.053 & 94 & 82 & 5 & -5 & -92 & 2.61E-7 & $3.11 \mathrm{E}-6$ & 3.30E-5 \\
\hline SF-539 & 0.703 & 2.388 & 2.354 & 2.212 & 0.913 & 0.667 & 0.042 & 98 & 90 & 12 & -5 & -94 & 3.26E-7 & $5.07 \mathrm{E}-6$ & 3.19E-5 \\
\hline SNB-19 & 0.459 & 1.858 & 1.771 & 1.688 & 0.847 & 0.757 & 0.226 & 94 & 88 & 28 & 21 & -51 & 4.26E-7 & $1.97 \mathrm{E}-5$ & $9.76 \mathrm{E}-5$ \\
\hline SNB-75 & 1.315 & 2.283 & 2.130 & 2.071 & 1.128 & 1.156 & 0.513 & 84 & 78 & -14 & -12 & -61 & $2.01 \mathrm{E}-7$ & $7.01 \mathrm{E}-7$ & $5.95 \mathrm{E}-5$ \\
\hline U251 & 0.270 & 1.674 & 1.576 & 1.545 & 0.623 & 0.518 & 0.091 & 93 & 91 & 25 & 18 & -66 & $4.18 \mathrm{E}-7$ & $1.62 \mathrm{E}-5$ & $6.37 \mathrm{E}-5$ \\
\hline Melanoma & & & & & & & & & & & & & & & \\
\hline LOX IM & 0.314 & 2.364 & 2.322 & 2.067 & 0.987 & 0.916 & 0.049 & 98 & 86 & 33 & 29 & -85 & $4.72 \mathrm{E}-7$ & $1.81 \mathrm{E}-5$ & 4.97E-5 \\
\hline MALME-3M & 0.623 & 1.493 & 1.446 & 1.297 & 1.027 & 1.094 & 0.385 & 95 & 77 & 46 & 54 & -38 & & $3.85 \mathrm{E}-5$ & $>1.00 \mathrm{E}-4$ \\
\hline & 0.457 & 1.980 & 1.954 & 1.666 & 0.801 & 0.634 & 0.07 & 98 & 79 & 23 & 12 & -85 & $3.29 \mathrm{E}-7$ & & $4.36 \mathrm{E}-5$ \\
\hline MDA-MB-435 & 0.554 & 2.655 & 2.510 & 1.451 & 0.490 & 0.397 & 0.068 & 93 & 43 & -12 & -28 & -88 & 7.17E-8 & $6.12 \mathrm{E}-7$ & $2.32 \mathrm{E}-5$ \\
\hline SK-MEL & 1.307 & 2.795 & 2.816 & 2.676 & 2.019 & 2.158 & 0.609 & 101 & 92 & 48 & 57 & -53 & & $3.29 \mathrm{E}-5$ & $9.31 \mathrm{E}-5$ \\
\hline SK-M & 0.602 & 1.970 & 1.939 & 1.785 & 1.295 & 1.251 & 0.174 & 98 & 87 & 51 & 47 & -71 & $1.58 \mathrm{E}-6$ & $2.51 \mathrm{E}-5$ & $6.63 \mathrm{E}-5$ \\
\hline SK-M & 1.055 & 3.298 & 3.295 & 3.250 & 1.845 & 1.308 & 0.005 & 100 & 98 & 35 & 11 & -100 & $5.80 \mathrm{E}-7$ & $1.26 \mathrm{E}-5$ & $3.57 \mathrm{E}-5$ \\
\hline UACC & 1.286 & 2.880 & 2.803 & 2.656 & 2.261 & 2.042 & 0.867 & 95 & 86 & 61 & 47 & -33 & $6.47 \mathrm{E}-6$ & $3.91 \mathrm{E}-5$ & $>1.00 \mathrm{E}-4$ \\
\hline UACC-62 & 0.616 & 2.444 & 2.277 & 1.824 & 0.907 & 0.807 & 0.042 & 91 & 66 & 16 & 10 & -93 & $2.09 \mathrm{E}-7$ & $1.26 \mathrm{E}-5$ & $3.83 E-5$ \\
\hline Ovarian Cancer & & & & & & & & & & & & & & & \\
\hline IGROV1 & 0.341 & 1.738 & 1.632 & 1.509 & 0.782 & 0.614 & 0.191 & 92 & 84 & 32 & 20 & -44 & $4.42 \mathrm{E}-7$ & $2.03 \mathrm{E}-5$ & $>1.00 \mathrm{E}-4$ \\
\hline & 0.52 & 1.874 & 1.952 & 1.761 & 0.727 & 0.651 & 0.084 & 106 & 92 & 15 & 9 & -84 & $3.48 \mathrm{E}-7$ & 1.25E-5 & $4.30 \mathrm{E}-5$ \\
\hline & 0.9 & 1.846 & 1.880 & 1.780 & 1.504 & 1.262 & 0.854 & 104 & 93 & 63 & 37 & -7 & $3.26 \mathrm{E}-6$ & $7.07 \mathrm{E}-5$ & $>1.00 \mathrm{E}-4$ \\
\hline OVCAF & 0.52 & 1.600 & 1.545 & 1.569 & 1.055 & 0.813 & 0.276 & 95 & 97 & 49 & 27 & -48 & 9.59 & E-5 & $>1.00 \mathrm{E}-4$ \\
\hline OVCAF & 0.43 & 2.408 & 2.420 & 2.362 & 1.385 & 0.977 & 0.423 & 101 & 98 & 48 & 27 & -4 & $9.12 \mathrm{E}-7$ & $7.63 \mathrm{E}-5$ & $>1.00 \mathrm{E}-4$ \\
\hline NCI/ADR-RES & 0.374 & 1.471 & 1.394 & 1.335 & 0.730 & 0.524 & 0.238 & 93 & 88 & 32 & 14 & -36 & $4.81 \mathrm{E}-7$ & $1.87 \mathrm{E}-5$ & $>1.00 \mathrm{E}-4$ \\
\hline SK-OV-3 & 0.781 & 1.884 & 1.883 & 1.707 & 1.207 & 1.237 & 0.688 & 100 & 84 & 39 & 41 & -12 & $5.61 \mathrm{E}-7$ & $5.96 \mathrm{E}-5$ & $>1.00 \mathrm{E}-4$ \\
\hline Renal Can & & & & & & & & & & & & & & & \\
\hline & 0.675 & 2.615 & 2.580 & 2.399 & 1.332 & 1.433 & 0.136 & 98 & 89 & 34 & 39 & -80 & & & $5.61 \mathrm{E}-5$ \\
\hline & 0.453 & 1.998 & 1.993 & 1.974 & 1.000 & 0.909 & 0.38 & 100 & 98 & 35 & 30 & -16 & & & $1.00 \mathrm{E}-4$ \\
\hline & 0.782 & 2.40 & 2.240 & 1.980 & 1.283 & 1.27 & 0.44 & 90 & 74 & 31 & 30 & -4 & & & $>1.00$ \\
\hline RXF & 0.99 & 1.68 & 1.617 & $1.4 \mathrm{c}$ & 1.021 & 0.9 & 0.13 & 91 & 73 & 4 & -5 & -8 & & & \\
\hline SN12C & 0.460 & 1.88 & 1.725 & 1.717 & 0.981 & 0.86 & 0.06 & 89 & 88 & 37 & 29 & -8 & & & 4.91 \\
\hline TK-1 & 0.852 & 1.941 & 1.814 & 1.773 & 1.563 & 1.381 & 0.75 & 88 & 85 & 65 & 49 & -11 & & & $>1.00 \mathrm{E}-4$ \\
\hline บO-31 & 0.759 & 2.484 & 2.290 & 2.223 & 1.431 & 1.220 & 0.074 & 89 & 85 & 39 & 27 & -90 & $5.75 \mathrm{E}-7$ & $1.69 \mathrm{E}-5$ & 4.53E-5 \\
\hline Prostate Cancer & & & & & & & & & & & & & & & \\
\hline & 0.503 & 2.051 & 1.938 & 1.676 & 0.921 & 0.760 & 0.266 & 93 & 76 & 27 & 17 & -47 & & $1.82 \mathrm{E}-5$ & $>1.00 \mathrm{E}-4$ \\
\hline DU-145 & 0.394 & 1.783 & 1.819 & 1.732 & 1.064 & 0.673 & 0.355 & 103 & 96 & 48 & 20 & -10 & $9.17 \mathrm{E}-7$ & $4.65 \mathrm{E}-5$ & $>1.00 \mathrm{E}-4$ \\
\hline Breast $\mathrm{Ca}$ & & & & & & & & & & & & & & & \\
\hline MCF7 & 0.400 & 2.224 & 2.032 & 1.763 & 0.634 & 0.616 & 0.289 & 89 & 75 & 13 & 12 & -28 & & & \\
\hline MDA-M & 0.569 & 1.452 & 1.46 & 1.387 & 1.047 & 0.803 & 0.174 & 102 & 93 & 54 & 27 & -70 & & & $6.26 \mathrm{E}-5$ \\
\hline HS 578T & 1.541 & 2.503 & 2.504 & 2.448 & 1.672 & 1.711 & 1.017 & 100 & 94 & 14 & 18 & -34 & 3.53 & & $>1.00 \mathrm{E}-4$ \\
\hline BT-54! & 1.345 & 2.471 & 2.481 & 2.316 & 1.737 & 1.666 & 0.28 & 101 & 86 & 35 & 28 & -7 & & & \\
\hline T-47D & 0.705 & 1.685 & 1.618 & 1.546 & 1.044 & 1.005 & 0.595 & 93 & 86 & 35 & 31 & -16 & $5.01 \mathrm{E}-7$ & 4.59E-5 & 1.00E-4 \\
\hline MDA-MB-468 & 0.760 & 1.725 & 1.600 & 1.466 & 0.825 & 0.783 & 0.376 & 87 & 73 & 7 & 2 & -51 & $2.23 \mathrm{E}-7$ & $1.11 \mathrm{E}-5$ & $9.77 \mathrm{E}-5$ \\
\hline
\end{tabular}


In-Vitro Testing Results

\begin{tabular}{|l|l|l|l|}
\hline NSC : D - 824743 / 1 & Experiment ID : 2009NS50 & Test Type : 08 & Units : Molar \\
\hline Report Date : March 24, 2021 & Test Date : September 28, 2020 & QNS : & MC: \\
\hline COMI : KLB2-32 & Stain Reagent : SRB Dual-Pass Related & SSPL : 1C1X & \\
\hline
\end{tabular}

Log10 Concentration

\begin{tabular}{|c|c|c|c|c|c|c|c|c|c|c|c|c|c|c|c|}
\hline \multirow[b]{2}{*}{ Panel/Cell Line } & \multirow{2}{*}{$\begin{array}{l}\text { Time } \\
\text { Zero }\end{array}$} & \multirow[b]{2}{*}{ Ctrl } & \multicolumn{5}{|c|}{ Mean Optical Densities } & \multicolumn{5}{|c|}{ Percent Growth } & \multirow[b]{2}{*}{ GI50 } & & \\
\hline & & & -8.0 & -7.0 & -6.0 & -5.0 & -4.0 & -8.0 & -7.0 & -6.0 & -5.0 & -4.0 & & TGI & LC50 \\
\hline Leukemia & & & & & & & & & & & & & & & \\
\hline CCRF-CEM & 0.425 & 1.963 & 1.924 & 1.454 & 0.633 & 0.618 & 0.397 & 97 & 67 & 13 & 13 & -7 & 2.07E-7 & $4.53 \mathrm{E}-5$ & $>1.00 \mathrm{E}-4$ \\
\hline HL-60(TB) & 0.585 & 2.674 & 2.490 & 1.868 & 0.540 & 0.437 & 0.419 & 91 & 61 & -8 & -25 & -28 & $1.46 \mathrm{E}-7$ & 7.74E-7 & $>1.00 \mathrm{E}-4$ \\
\hline K-562 & 0.154 & 1.484 & 1.417 & 0.506 & 0.273 & 0.266 & 0.154 & 95 & 26 & 9 & 8 & & $4.53 \mathrm{E}-8$ & $>1.00 \mathrm{E}-4$ & $>1.00 \mathrm{E}-4$ \\
\hline MOLT-4 & 0.589 & 2.702 & 2.692 & 2.445 & 1.000 & 0.803 & 0.444 & 100 & 88 & 19 & 10 & -25 & 3.57E-7 & $1.95 \mathrm{E}-5$ & $>1.00 \mathrm{E}-4$ \\
\hline SR & 0.269 & 0.753 & 0.740 & 0.411 & 0.349 & 0.287 & 0.192 & 97 & 29 & 16 & 4 & -29 & $4.95 \mathrm{E}-8$ & $1.29 \mathrm{E}-5$ & $>1.00 \mathrm{E}-4$ \\
\hline Non-Small Cell Lung & Cancer & & & & & & & & & & & & & & \\
\hline A549/ATCC & 0.452 & 2.381 & 2.235 & 1.926 & 0.916 & 0.685 & 0.298 & 92 & 76 & 24 & 12 & -34 & $3.19 \mathrm{E}-7$ & $1.82 \mathrm{E}-5$ & $>1.00 \mathrm{E}-4$ \\
\hline EKVX & 0.611 & 1.911 & 1.886 & 1.830 & 1.042 & 1.105 & 0.470 & 98 & 94 & 33 & 38 & -23 & $5.26 \mathrm{E}-7$ & $4.18 \mathrm{E}-5$ & $>1.00 \mathrm{E}-4$ \\
\hline HOP-62 & 1.085 & 2.947 & 2.839 & 2.444 & 1.719 & 1.650 & 0.349 & 94 & 73 & 34 & 30 & -68 & 3.89E-7 & $2.04 \mathrm{E}-5$ & $6.58 \mathrm{E}-5$ \\
\hline HOP-92 & 1.137 & 1.648 & 1.588 & 1.526 & 1.526 & 1.296 & 0.213 & 88 & 76 & 76 & 31 & -81 & $3.79 \mathrm{E}-6$ & $1.89 \mathrm{E}-5$ & $5.27 \mathrm{E}-5$ \\
\hline $\mathrm{NCl}-\mathrm{H} 226$ & 0.796 & 1.508 & 1.438 & 1.443 & 0.992 & 0.810 & 0.204 & 90 & 91 & 28 & 2 & -74 & $4.41 \mathrm{E}-7$ & $1.06 \mathrm{E}-5$ & $4.79 \mathrm{E}-5$ \\
\hline $\mathrm{NCl}-\mathrm{H} 23$ & 0.699 & 2.028 & 1.967 & 1.847 & 1.082 & 0.887 & 0.197 & 95 & 86 & 29 & 14 & -72 & $4.28 \mathrm{E}-7$ & $1.46 \mathrm{E}-5$ & $5.57 \mathrm{E}-5$ \\
\hline $\mathrm{NCl}-\mathrm{H} 322 \mathrm{M}$ & 0.919 & 2.368 & 2.287 & 2.286 & 1.482 & 1.519 & 0.442 & 94 & 94 & 39 & 41 & -52 & $6.29 \mathrm{E}-7$ & $2.78 \mathrm{E}-5$ & $9.54 \mathrm{E}-5$ \\
\hline $\mathrm{NCl}-\mathrm{H} 460$ & 0.319 & 2.976 & 3.016 & 2.716 & 0.565 & 0.357 & 0.091 & 102 & 90 & 9 & 1 & -72 & $3.14 \mathrm{E}-7$ & $1.05 \mathrm{E}-5$ & $5.06 \mathrm{E}-5$ \\
\hline $\mathrm{NCl}-\mathrm{H} 522$ & 1.273 & 3.016 & 2.862 & 2.467 & 1.809 & 1.667 & 0.628 & 91 & 69 & 31 & 23 & -51 & 3.09E-7 & 2.03E-5 & $9.78 \mathrm{E}-5$ \\
\hline Colon Cancer & & & & & & & & & & & & & & & \\
\hline COLO 205 & 0.671 & 2.437 & 2.505 & 2.312 & 0.943 & 0.734 & 0.328 & 104 & 93 & 15 & 4 & -51 & $3.58 \mathrm{E}-7$ & $1.16 \mathrm{E}-5$ & $9.54 \mathrm{E}-5$ \\
\hline HCC-2998 & 0.410 & 1.368 & 1.163 & 1.286 & 0.731 & 0.489 & 0.058 & 79 & 91 & 34 & 8 & -86 & $5.19 \mathrm{E}-7$ & $1.22 \mathrm{E}-5$ & $4.15 \mathrm{E}-5$ \\
\hline HCT-116 & 0.243 & 2.348 & 2.123 & 1.661 & 0.679 & 0.355 & 0.041 & 89 & 67 & 21 & 5 & -83 & 2.36E-7 & $1.15 \mathrm{E}-5$ & $4.21 \mathrm{E}-5$ \\
\hline HCT-15 & 0.254 & 1.749 & 1.744 & 0.951 & 0.556 & 0.370 & 0.060 & 100 & 47 & 20 & 8 & -76 & $8.64 \mathrm{E}-8$ & $1.24 \mathrm{E}-5$ & $4.86 \mathrm{E}-5$ \\
\hline HT29 & 0.309 & 2.130 & 2.162 & 1.671 & 0.443 & 0.439 & 0.137 & 102 & 75 & 7 & 7 & -56 & $2.33 \mathrm{E}-7$ & $1.30 \mathrm{E}-5$ & $8.12 \mathrm{E}-5$ \\
\hline KM12 & 0.511 & 2.553 & 2.529 & 1.669 & 0.906 & 0.700 & 0.137 & 99 & 57 & 19 & 9 & -73 & $1.51 \mathrm{E}-7$ & $1.29 \mathrm{E}-5$ & $5.23 \mathrm{E}-5$ \\
\hline SW-620 & 0.291 & 2.192 & 2.164 & 1.296 & 0.622 & 0.587 & 0.063 & 99 & 53 & 17 & 16 & -78 & $1.20 \mathrm{E}-7$ & $1.46 \mathrm{E}-5$ & 4.99E-5 \\
\hline CNS Cancer & & & & & & & & & & & & & & & \\
\hline SF-268 & 1.025 & 2.536 & 2.434 & 2.201 & 1.889 & 1.305 & 0.378 & 93 & 78 & 57 & 19 & -63 & $1.53 \mathrm{E}-6$ & $1.69 \mathrm{E}-5$ & $6.91 \mathrm{E}-5$ \\
\hline SF-295 & 0.622 & 2.258 & 2.081 & 1.206 & 0.666 & 0.687 & 0.037 & 89 & 36 & 3 & 4 & -94 & $5.40 \mathrm{E}-8$ & 1.10E-5 & $3.55 \mathrm{E}-5$ \\
\hline SF-539 & 0.527 & 1.917 & 1.921 & 1.502 & 0.568 & 0.529 & 0.074 & 100 & 70 & 3 & & -86 & $2.00 \mathrm{E}-7$ & $1.00 \mathrm{E}-5$ & $3.82 \mathrm{E}-5$ \\
\hline SNB-19 & 0.646 & 2.242 & 2.166 & 1.883 & 1.067 & 1.035 & 0.101 & 95 & 77 & 26 & 24 & -84 & $3.45 \mathrm{E}-7$ & 1.67E-5 & $4.82 \mathrm{E}-5$ \\
\hline SNB-75 & 1.147 & 2.058 & 1.857 & 1.687 & 1.052 & 1.160 & 0.227 & 78 & 59 & -8 & 1 & -80 & 1.37E-7 & & $4.26 \mathrm{E}-5$ \\
\hline U251 & 0.453 & 2.121 & 1.993 & 1.702 & 0.758 & 0.708 & 0.064 & 92 & 75 & 18 & 15 & -86 & $2.75 \mathrm{E}-7$ & $1.41 \mathrm{E}-5$ & $4.41 \mathrm{E}-5$ \\
\hline Melanoma & & & & & & & & & & & & & & & \\
\hline LOX IMVI & 0.281 & 1.793 & 1.780 & 1.325 & 0.875 & 0.460 & 0.043 & 99 & 69 & 39 & 12 & -85 & $4.36 \mathrm{E}-7$ & $1.33 \mathrm{E}-5$ & $4.36 \mathrm{E}-5$ \\
\hline MALME-3M & 0.751 & 1.619 & 1.554 & 1.294 & 1.132 & 1.237 & 0.197 & 92 & 63 & 44 & 56 & -74 & & $2.70 \mathrm{E}-5$ & $6.55 \mathrm{E}-5$ \\
\hline M14 & 0.536 & 2.212 & 2.073 & 1.479 & 0.518 & 0.784 & 0.119 & 92 & 56 & -3 & 15 & -78 & 1.27E-7 & & $5.01 \mathrm{E}-5$ \\
\hline MDA-MB-435 & 0.836 & 2.371 & 2.226 & 1.552 & 1.345 & 1.161 & 0.077 & 91 & 47 & 33 & 21 & -91 & $8.38 \mathrm{E}-8$ & $1.55 \mathrm{E}-5$ & $4.32 \mathrm{E}-5$ \\
\hline SK-MEL-2 & 0.913 & 2.301 & 2.237 & 1.945 & 1.572 & 1.511 & 0.283 & 95 & 74 & 47 & 43 & -69 & 8.04E-7 & $2.42 \mathrm{E}-5$ & $6.77 \mathrm{E}-5$ \\
\hline SK-MEL-28 & 0.545 & 1.773 & 1.784 & 1.415 & 1.273 & 1.166 & 0.095 & 101 & 71 & 59 & 51 & -83 & $1.01 \mathrm{E}-5$ & $2.40 \mathrm{E}-5$ & $5.69 \mathrm{E}-5$ \\
\hline SK-MEL-5 & 1.093 & 3.163 & 3.122 & 2.689 & 1.644 & 0.929 & 0.026 & 98 & 77 & 27 & -15 & -98 & $3.44 \mathrm{E}-7$ & $4.35 \mathrm{E}-6$ & $2.65 \mathrm{E}-5$ \\
\hline UACC-257 & 0.814 & 2.143 & 2.053 & 1.772 & 1.659 & 1.320 & 0.331 & 93 & 72 & 64 & 38 & -59 & $3.41 \mathrm{E}-6$ & $2.46 \mathrm{E}-5$ & $8.01 \mathrm{E}-5$ \\
\hline UACC-62 & 0.745 & 2.627 & 2.460 & 1.472 & 1.235 & 1.162 & 0.056 & 91 & 39 & 26 & 22 & -92 & $6.07 \mathrm{E}-8$ & $1.56 \mathrm{E}-5$ & $4.26 \mathrm{E}-5$ \\
\hline Ovarian Cancer & & & & & & & & & & & & & & & \\
\hline IGROV1 & 0.635 & 2.349 & 2.319 & 1.813 & 1.166 & 0.864 & 0.192 & 98 & 69 & 31 & 13 & -70 & 3.13E-7 & $1.45 \mathrm{E}-5$ & $5.77 \mathrm{E}-5$ \\
\hline OVCAR-3 & 0.560 & 1.783 & 1.772 & 1.079 & 0.576 & 0.539 & 0.076 & 99 & 42 & 1 & -4 & -87 & $7.34 \mathrm{E}-8$ & $1.81 \mathrm{E}-6$ & $3.62 \mathrm{E}-5$ \\
\hline OVCAR-4 & 0.924 & 1.870 & 1.874 & 1.766 & 1.490 & 1.267 & 0.195 & 100 & 89 & 60 & 36 & -79 & $2.61 \mathrm{E}-6$ & $2.06 \mathrm{E}-5$ & $5.61 \mathrm{E}-5$ \\
\hline OVCAR-5 & 0.469 & 1.499 & 1.475 & 1.376 & 0.794 & 0.681 & 0.071 & 98 & 88 & 32 & 21 & -85 & $4.71 \mathrm{E}-7$ & $1.57 \mathrm{E}-5$ & $4.67 \mathrm{E}-5$ \\
\hline OVCAR-8 & 0.492 & 2.329 & 2.261 & 2.211 & 0.931 & 0.667 & 0.239 & 96 & 94 & 24 & 10 & -51 & $4.22 \mathrm{E}-7$ & $1.43 \mathrm{E}-5$ & $9.48 \mathrm{E}-5$ \\
\hline NCI/ADR-RES & 0.402 & 1.401 & 1.400 & 1.038 & 0.534 & 0.475 & 0.162 & 100 & 64 & 13 & 7 & -60 & $1.86 \mathrm{E}-7$ & $1.29 \mathrm{E}-5$ & 7.17E-5 \\
\hline SK-OV-3 & 1.128 & 2.277 & 2.243 & 2.150 & 1.810 & 1.817 & 0.505 & 97 & 89 & 59 & 60 & -55 & $1.22 \mathrm{E}-5$ & $3.31 \mathrm{E}-5$ & $9.00 \mathrm{E}-5$ \\
\hline Renal Cancer & & & & & & & & & & & & & & & \\
\hline $786-0$ & 0.679 & 2.478 & 2.372 & 2.238 & 1.286 & 1.107 & 0.166 & 94 & 87 & 34 & 24 & -76 & 4.93E-7 & $1.73 E-5$ & $5.53 E-5$ \\
\hline A498 & 1.155 & 2.033 & 2.020 & 1.514 & 1.141 & 1.073 & 0.052 & 99 & 41 & -1 & -7 & -96 & $6.95 \mathrm{E}-8$ & $9.34 \mathrm{E}-7$ & 3.06E-5 \\
\hline $\mathrm{ACHN}$ & 0.368 & 1.665 & 1.675 & 1.336 & 0.892 & 0.643 & 0.222 & 101 & 75 & 40 & 21 & -40 & $5.24 \mathrm{E}-7$ & $2.23 \mathrm{E}-5$ & $>1.00 \mathrm{E}-4$ \\
\hline CAKI-1 & 0.692 & 2.145 & 2.018 & 0.995 & 0.701 & 0.741 & 0.032 & 91 & 21 & 1 & 3 & -95 & $3.85 \mathrm{E}-8$ & $1.08 \mathrm{E}-5$ & 3.47E-5 \\
\hline RXF 393 & 0.969 & 1.529 & 1.482 & 1.295 & 0.808 & 1.016 & 0.203 & 92 & 58 & -17 & 8 & -79 & $1.29 \mathrm{E}-7$ & & $4.65 \mathrm{E}-5$ \\
\hline SN12C & 0.541 & 2.157 & 2.001 & 1.974 & 1.143 & 0.912 & 0.083 & 90 & 89 & 37 & 23 & -85 & $5.65 \mathrm{E}-7$ & $1.63 \mathrm{E}-5$ & 4.76E-5 \\
\hline TK-10 & 0.980 & 2.007 & 1.887 & 1.898 & 1.667 & 1.577 & 0.501 & 88 & 89 & 67 & 58 & -49 & $1.19 \mathrm{E}-5$ & $3.49 \mathrm{E}-5$ & $>1.00 \mathrm{E}-4$ \\
\hline UO-31 & 0.612 & 1.957 & 1.792 & 1.639 & 1.154 & 0.999 & 0.020 & 88 & 76 & 40 & 29 & -97 & $5.38 \mathrm{E}-7$ & $1.70 \mathrm{E}-5$ & $4.24 \mathrm{E}-5$ \\
\hline Prostate Cancer & & & & & & & & & & & & & & & \\
\hline PC-3 & 0.454 & 1.764 & 1.736 & 1.307 & 0.677 & 0.592 & 0.107 & 98 & 65 & 17 & 10 & -76 & $2.06 \mathrm{E}-7$ & $1.32 \mathrm{E}-5$ & 4.97E-5 \\
\hline DU-145 & 0.408 & 1.766 & 1.825 & 1.679 & 0.660 & 0.542 & 0.191 & 104 & 94 & 19 & 10 & -53 & $3.80 \mathrm{E}-7$ & $1.43 E-5$ & $8.86 \mathrm{E}-5$ \\
\hline Breast Cancer & & & & & & & & & & & & & & & \\
\hline MCF7 & 0.332 & 1.898 & 1.818 & 0.799 & 0.512 & 0.420 & 0.201 & 95 & 30 & 11 & 6 & -39 & $4.89 \mathrm{E}-8$ & $1.33 \mathrm{E}-5$ & $>1.00 \mathrm{E}-4$ \\
\hline MDA-MB-231/ATCC & 0.429 & 1.155 & 1.197 & 1.170 & 0.722 & 0.610 & 0.118 & 106 & 102 & 40 & 25 & -72 & $6.99 \mathrm{E}-7$ & $1.80 \mathrm{E}-5$ & $5.87 \mathrm{E}-5$ \\
\hline HS 578T & 1.505 & 2.499 & 2.426 & 2.168 & 1.634 & 1.611 & 1.029 & 93 & 67 & 13 & 11 & -32 & $2.04 \mathrm{E}-7$ & $1.79 \mathrm{E}-5$ & $>1.00 \mathrm{E}-4$ \\
\hline BT-549 & 1.085 & 2.204 & 2.170 & 1.929 & 1.645 & 1.316 & 0.264 & 97 & 75 & 50 & 21 & -76 & $9.99 \mathrm{E}-7$ & $1.64 \mathrm{E}-5$ & $5.41 \mathrm{E}-5$ \\
\hline $\mathrm{T}-47 \mathrm{D}$ & 1.153 & 2.602 & 2.359 & 2.126 & 1.655 & 1.658 & 0.921 & 83 & 67 & 35 & 35 & -20 & 3.36E-7 & $4.30 \mathrm{E}-5$ & $>1.00 \mathrm{E}-4$ \\
\hline MDA-MB-468 & 0.815 & 1.684 & 1.643 & 1.212 & 0.874 & 0.904 & 0.434 & 95 & 46 & 7 & 10 & -47 & $8.19 \mathrm{E}-8$ & $1.51 \mathrm{E}-5$ & $>1.00 \mathrm{E}-4$ \\
\hline
\end{tabular}


In-Vitro Testing Results

\begin{tabular}{|l|l|l|l|}
\hline NSC : D - 824744 / 1 & Experiment ID : 2009NS50 & Test Type : 08 & Units : Molar \\
\hline Report Date : March 24, 2021 & Test Date : September 28, 2020 & QNS : & MC : \\
\hline COMI : KLB2-33 & Stain Reagent : SRB Dual-Pass Related & SSPL : 1C1X & \\
\hline
\end{tabular}

Log10 Concentration

\begin{tabular}{|c|c|c|c|c|c|c|c|c|c|c|c|c|c|c|c|}
\hline \multirow[b]{2}{*}{ Panel/Cell Line } & \multirow{2}{*}{$\begin{array}{l}\text { Time } \\
\text { Zero }\end{array}$} & \multirow[b]{2}{*}{ Ctrl } & \multicolumn{5}{|c|}{ Mean Optical Densities } & \multicolumn{5}{|c|}{ Percent Growth } & \multirow[b]{2}{*}{ GI50 } & \multirow[b]{2}{*}{ TGI } & \\
\hline & & & -8.0 & -7.0 & -6.0 & -5.0 & -4.0 & -8.0 & -7.0 & -6.0 & -5.0 & -4.0 & & & LC50 \\
\hline Leukemia & & & & & & & & & & & & & & & \\
\hline CCRF-CEM & 0.425 & 2.027 & $\begin{array}{r}2.030 \\
2.383\end{array}$ & 0.874 & 0.624 & 0.636 & 0.474 & 100 & 28 & 12 & 13 & 3 & 4.96E-8 & $>1.00 \mathrm{E}-4$ & $>1.00 \mathrm{E}-4$ \\
\hline HL-60(TB) & 0.585 & 2.547 & 2.383 & 0.862 & 0.518 & 0.403 & 0.351 & 92 & 14 & -11 & -31 & -40 & 3.44E-8 & 3.57E-7 & $>1.00 \mathrm{E}-4$ \\
\hline K-562 & 0.154 & 1.365 & $\begin{array}{l}1.305 \\
2709\end{array}$ & 0.316 & 0.254 & 0.244 & 0.186 & 95 & 13 & 8 & 7 & $\begin{array}{r}3 \\
13\end{array}$ & 3.56E-8 & $>1.00 \mathrm{E}-4$ & $>1.00 \mathrm{E}-4$ \\
\hline $\begin{array}{l}\text { MOLT-4 } \\
\text { SR }\end{array}$ & $\begin{array}{l}0.589 \\
0.269\end{array}$ & $\begin{array}{l}2.686 \\
0.703\end{array}$ & $\begin{array}{l}2.709 \\
0.729\end{array}$ & $\begin{array}{l}1.474 \\
0385\end{array}$ & 0.916 & $\begin{array}{l}0.752 \\
0.305\end{array}$ & $\begin{array}{l}0.510 \\
0.255\end{array}$ & $\begin{array}{l}101 \\
106\end{array}$ & 42 & 16 & 8 & -13 & $\begin{array}{l}7.37 \mathrm{E}-8 \\
506 \mathrm{~F}-8\end{array}$ & $2.33 \mathrm{E}-5$ & $>1.00 \mathrm{E}-4$ \\
\hline Non-Small Cell Lung & Cancer & & & & & & & & & & & & & & \\
\hline A549/ATCC & 0.452 & 2.298 & 2.184 & 1.382 & 0.879 & 0.698 & 0.489 & 94 & 50 & 23 & 13 & 2 & $1.03 \mathrm{E}-7$ & $>1.00 \mathrm{E}-4$ & $>1.00 \mathrm{E}-4$ \\
\hline EKVX & 0.611 & 1.937 & 1.882 & 1.572 & 1.056 & 1.149 & 0.636 & 96 & 72 & 34 & 41 & 2 & $3.78 \mathrm{E}-7$ & $>1.00 \mathrm{E}-4$ & $>1.00 \mathrm{E}-4$ \\
\hline HOP-62 & 1.085 & 2.868 & 2.777 & 1.933 & 1.669 & 1.576 & 1.032 & 95 & 48 & 33 & 28 & -5 & $8.88 \mathrm{E}-8$ & 7.07E-5 & $>1.00 \mathrm{E}-4$ \\
\hline HOP-92 & 1.137 & 1.709 & 1.649 & 1.568 & 1.598 & 1.300 & 0.931 & 90 & 75 & 81 & 28 & -18 & 3.85E-6 & 4.07E-5 & $>1.00 \mathrm{E}-4$ \\
\hline $\mathrm{NCl}-\mathrm{H} 226$ & 0.796 & 1.478 & 1.451 & 1.284 & 0.958 & 0.826 & 0.593 & 96 & 72 & 24 & 4 & -26 & 2.82E-7 & 1.40E-5 & $>1.00 \mathrm{E}-4$ \\
\hline $\mathrm{NCl}-\mathrm{H} 23$ & 0.699 & 2.109 & 2.010 & 1.580 & 1.157 & 1.008 & 0.675 & 93 & 62 & 32 & 22 & -4 & 2.61E-7 & 7.28E-5 & $>1.00 \mathrm{E}-4$ \\
\hline $\mathrm{NCl}-\mathrm{H} 322 \mathrm{M}$ & 0.919 & 2.374 & 2.322 & 1.987 & 1.522 & 1.582 & 1.105 & 96 & 73 & 41 & 46 & 13 & $5.40 \mathrm{E}-7$ & $>1.00 \mathrm{E}-4$ & $>1.00 \mathrm{E}-4$ \\
\hline $\mathrm{NCl}-\mathrm{H} 460$ & 0.319 & 2.988 & 2.975 & 1.035 & 0.565 & 0.348 & 0.218 & 100 & 27 & 9 & 1 & -32 & 4.80E-8 & 1.08E-5 & $>1.00 \mathrm{E}-4$ \\
\hline NCl-H522 & 1.273 & 2.963 & 2.803 & 1.968 & 1.641 & 1.465 & 1.052 & 91 & 41 & 22 & 11 & -17 & 6.61E-8 & $2.48 \mathrm{E}-5$ & $>1.00 \mathrm{E}-4$ \\
\hline Colon Cancer & & & & & & & & & & & & & & & \\
\hline COLO 205 & 0.671 & 2.291 & 2.315 & 1.461 & 0.659 & 0.397 & 0.353 & 101 & 49 & -2 & -41 & -47 & $9.46 \mathrm{E}-8$ & $9.19 \mathrm{E}-7$ & $>1.00 \mathrm{E}-4$ \\
\hline HCC-2998 & 0.410 & 1.421 & 1.333 & 1.090 & 0.582 & 0.487 & 0.284 & 91 & 67 & 17 & 8 & -31 & 2.20E-7 & $1.58 \mathrm{E}-5$ & $>1.00 \mathrm{E}-4$ \\
\hline HCT-116 & 0.243 & $\begin{array}{l}2.248 \\
1.757\end{array}$ & 2.257 & 0.834 & 0.640 & 0.386 & 0.261 & 100 & 29 & 20 & 7 & 1 & $5.14 \mathrm{E}-8$ & $>1.00 \mathrm{E}-4$ & $>1.00 \mathrm{E}-4$ \\
\hline $\begin{array}{l}\text { HCT-15 } \\
\text { HT29 }\end{array}$ & 0.254 & 1.757 & $\begin{array}{l}1.671 \\
\end{array}$ & 0.699 & 0.498 & 0.336 & 0.221 & 94 & 30 & 16 & 5 & -13 & 4.84E-8 & $1.96 \mathrm{E}-5$ & $>1.00 \mathrm{E}-4$ \\
\hline $\begin{array}{l}\mathrm{HT} 2 \mathrm{~S} \\
\mathrm{KM} 1\end{array}$ & 0.309 & 2.095 & 2.117 & 0.676 & 0.430 & 0.411 & 0.240 & 101 & 21 & 7 & 6 & -22 & 4.31E-8 & 1.60E-5 & $>1.00 \mathrm{E}-4$ \\
\hline $\begin{array}{l}\text { KM12 } \\
\text { SW-620 }\end{array}$ & 0.511 & $\begin{array}{l}2.630 \\
2.214\end{array}$ & $\begin{array}{l}2.566 \\
2.137\end{array}$ & 1.062 & 0.864 & 0.573 & $\begin{array}{l}0.334 \\
0.391\end{array}$ & $\begin{array}{l}97 \\
96\end{array}$ & 26 & $\begin{array}{l}17 \\
19\end{array}$ & 3 & -35 & 4.59E-8 & 1.20E-5 & $>1.00 \mathrm{E}-4$ \\
\hline & 0.201 & 2.214 & & 0.145 & 0.049 & 0.004 & & & & 19 & 10 & & $4.0<5-0$ & $>1.00 E-4$ & $>1.00 \mathrm{E}-4$ \\
\hline $\begin{array}{l}\text { CNS Cancer } \\
\text { SE-268 }\end{array}$ & 1025 & & & & 1892 & 1252 & 0.944 & 90 & & 54 & 14 & & 1 29E_6 & $4105-5$ & $>100 E_{-4}$ \\
\hline SF-295 & 0.622 & 2.277 & $\begin{array}{l}2.106 \\
2.406\end{array}$ & $\begin{array}{l}1.940 \\
0.869\end{array}$ & 0.621 & 0.663 & 0.371 & 90 & 15 & 34 & 2 & $\begin{array}{l}-0 \\
-40\end{array}$ & $3.39 \mathrm{E}-8$ & $4.40 E-5$ & $>1.00 \mathrm{E}-4$ \\
\hline SF-539 & 0.527 & 1.986 & 1.948 & 1.066 & 0.561 & 0.521 & 0.285 & 97 & 37 & 2 & -1 & -46 & 6.07E-8 & $4.66 \mathrm{E}-6$ & $>1.00 \mathrm{E}-4$ \\
\hline SNB-19 & 0.646 & 2.333 & 2.273 & 1.404 & 1.093 & 1.094 & 0.844 & 96 & 45 & 26 & 27 & 12 & 7.97E-8 & $>1.00 \mathrm{E}-4$ & $>1.00 \mathrm{E}-4$ \\
\hline SNB-75 & 1.147 & 1.981 & 1.806 & 1.212 & 1.014 & 1.142 & 0.970 & 79 & 8 & -12 & & -15 & 2.55E-8 & 2.52E-7 & $>1.00 \mathrm{E}-4$ \\
\hline U251 & 0.453 & 2.095 & 1.982 & 1.089 & 0.771 & 0.738 & 0.296 & 93 & 39 & 19 & 17 & -35 & $6.20 \mathrm{E}-8$ & $2.16 \mathrm{E}-5$ & $>1.00 \mathrm{E}-4$ \\
\hline Melanoma & & & & & & & & & & & & & & & \\
\hline LOXIMVI & 0.281 & 1.901 & 1.885 & 0.976 & 0.892 & 0.512 & 0.158 & 99 & 43 & 38 & 14 & -44 & 7.46E-8 & 1.76E- 5 & $>1.00 \mathrm{E}-4$ \\
\hline MALME & 0.751 & 1.587 & 1.554 & 1.157 & 1.193 & 1.160 & 0.595 & 96 & 49 & 53 & 49 & -21 & & 5.02E-5 & $>1.00 \mathrm{E}-4$ \\
\hline M14 & 0.536 & 2.240 & 2.081 & 1.200 & 0.569 & 0.776 & 0.564 & 91 & 39 & 2 & 14 & 2 & $6.12 \mathrm{E}-8$ & $>1.00 \mathrm{E}-4$ & $>1.00 \mathrm{E}-4$ \\
\hline MDA-MB-435 & 0.836 & 2.331 & 2.086 & 1.327 & 1.339 & 1.108 & 0.723 & 84 & 33 & 34 & 18 & -14 & $4.59 \mathrm{E}-8$ & 3.74E-5 & $>1.00 \mathrm{E}-4$ \\
\hline SK-MEL-2 & 0.913 & 2.257 & 2.163 & 1.568 & 1.495 & 1.391 & 0.821 & 93 & 49 & 43 & 36 & -10 & $9.36 \mathrm{E}-8$ & $6.01 \mathrm{E}-5$ & $>1.00 \mathrm{E}-4$ \\
\hline SK-MEL- & 0.545 & 1.821 & 1.818 & 1.322 & 1.256 & 1.165 & 0.585 & 100 & 61 & 56 & 49 & 3 & $6.38 \mathrm{E}-6$ & $>1.00 \mathrm{E}-4$ & $>1.00 \mathrm{E}-4$ \\
\hline SK-MEL- & 1.093 & 3.210 & 3.193 & 2.220 & 1.842 & 0.841 & 0.026 & 99 & 53 & 35 & -23 & -98 & $1.51 \mathrm{E}-7$ & 4.03E-6 & $2.30 \mathrm{E}-5$ \\
\hline UACC- 257 & 0.814 & 2.078 & 1.970 & 1.495 & 1.637 & 1.396 & 0.620 & 91 & 54 & 65 & 46 & -24 & $6.22 \mathrm{E}-6$ & $4.55 \mathrm{E}-5$ & $>1.00 \mathrm{E}-4$ \\
\hline UACC- 62 & 0.745 & 2.677 & 2.473 & 1.222 & 1.192 & 1.146 & 0.589 & 89 & 25 & 23 & 21 & -21 & 4.06E-8 & 3.14E-5 & $>1.00 \mathrm{E}-4$ \\
\hline Ovarian Cancer & & & & & & & & & & & & & & & \\
\hline & 0.635 & 2.332 & 2.318 & 1.484 & 1.166 & 0.848 & 0.693 & 99 & 50 & 31 & 13 & 3 & $1.00 \mathrm{E}-7$ & $>1.00 \mathrm{E}-4$ & $>1.00 \mathrm{E}-4$ \\
\hline & 0.560 & 1.800 & 1.850 & 0.740 & 0.533 & 0.535 & 0.23 & 104 & 15 & -5 & -4 & -58 & & & $7.08 \mathrm{E}-5$ \\
\hline & 0.924 & 1.841 & 1.881 & 1.587 & 1.471 & 1.223 & 0.921 & 104 & 72 & 60 & 33 & & & & $>1.00 \mathrm{E}-4$ \\
\hline & 0.46 & 1.5 & 1.507 & 1.261 & 0.774 & 0.712 & 0.5 & 98 & 75 & 29 & 23 & 11 & & $>1.0$ & $>1.00 \mathrm{E}-4$ \\
\hline & 0.492 & 2.32 & 2.237 & 1.601 & 0.862 & 0.674 & 0.45 & 95 & 61 & 20 & 10 & -8 & & & $>1.0$ \\
\hline NCI/ADR-RES & 0.402 & 1.384 & 1.430 & 0.820 & 0.569 & 0.528 & 0.3 & 105 & 43 & 17 & 13 & -13 & & & $>1.00 \mathrm{E}-4$ \\
\hline SK-OV-3 & 1.128 & 2.179 & 2.211 & 1.881 & 1.690 & 1.712 & 1.068 & 103 & 72 & 53 & 56 & -5 & $1.23 \mathrm{E}-5$ & $8.18 \mathrm{E}-5$ & $>1.00 \mathrm{E}-4$ \\
\hline Renal Cancer & & & & & & & & & & & & & & & \\
\hline & 0.679 & 2.521 & 2.402 & 1.489 & 1.354 & 1.070 & 0.735 & 93 & 44 & 37 & 21 & 3 & & $>1.00 \mathrm{E}-4$ & $>1.00 \mathrm{E}-4$ \\
\hline A498 & 1.155 & 2.024 & 1.944 & 1.270 & 1.152 & 1.03 & 1.039 & 91 & 13 & & -10 & -10 & & 9.5 & $>1.00 \mathrm{E}-4$ \\
\hline $\mathrm{ACHN}$ & 0.368 & 1.694 & 1.700 & 0.956 & 0.886 & 0.653 & 0.371 & 100 & 44 & 39 & 21 & & $7.92 \mathrm{E}-8$ & $>1.00 \mathrm{E}-4$ & $>1.00 \mathrm{E}-4$ \\
\hline & 0.692 & 2.153 & 1.95 & 0.8 & 0.788 & 0.64 & 0.61 & 86 & 10 & 7 & -7 & -11 & & $3.02 \mathrm{E}-6$ & $>1.00 \mathrm{E}-4$ \\
\hline & 0.96 & 1.552 & 1.51 & 1.2 & 0.882 & 1.08 & 0.8 & 94 & 42 & -9 & 20 & -8 & $7.12 \mathrm{E}-8$ & & $>1.00 \mathrm{E}-4$ \\
\hline SN12 & 0.541 & 2.221 & 2.00 & 1.630 & 1.172 & $0.8 \mathrm{~s}$ & 0.50 & 87 & 65 & 38 & 21 & -7 & $3.50 \mathrm{E}-7$ & 5.61E-5 & $>1.00 \mathrm{E}-4$ \\
\hline TK-10 & 0.980 & 1.955 & 1.837 & 1.791 & 1.662 & 1.553 & 1.039 & 88 & 83 & 70 & 59 & 6 & $1.46 \mathrm{E}-5$ & $>1.00 \mathrm{E}-4$ & $>1.00 \mathrm{E}-4$ \\
\hline บO-31 & 0.612 & 1.970 & 1.779 & 1.322 & 1.171 & 1.010 & 0.619 & 86 & 52 & 41 & 29 & & $1.59 \mathrm{E}-7$ & $>1.00 \mathrm{E}-4$ & $>1.00 \mathrm{E}-4$ \\
\hline Prostate Cancer & & & & & & & & & & & & & & & \\
\hline & 0.454 & 1.821 & 1.828 & 0.967 & 0.716 & 0.646 & 0.477 & 100 & 37 & 19 & 14 & 2 & $6.33 \mathrm{E}-8$ & $>1.00 \mathrm{E}-4$ & $>1.00 \mathrm{E}-4$ \\
\hline DU-145 & 0.408 & 1.783 & 1.836 & 1.364 & 0.655 & 0.472 & 0.435 & 104 & 70 & 18 & 5 & 2 & $2.39 \mathrm{E}-7$ & $>1.00 \mathrm{E}-4$ & $>1.00 \mathrm{E}-4$ \\
\hline Breast Cancer & & & & & & & & & & & & & & & \\
\hline MCF & 0.332 & 1.889 & 1.762 & 0.547 & 0.496 & 0.419 & 0.29 & 92 & 14 & 11 & 6 & -10 & & & \\
\hline MDA-N & 0.429 & 1.195 & 1.214 & 1.044 & 0.712 & 0.626 & 0.44 & 102 & 80 & 37 & 26 & & $4.99 \mathrm{E}-7$ & $>1.00 \mathrm{E}-4$ & $>1.00 \mathrm{E}-4$ \\
\hline HS 57 & 1.505 & 2.432 & 2.298 & 1.665 & 1.655 & 1.584 & 1.44 & 86 & 17 & 16 & 9 & -4 & $3.32 \mathrm{E}-8$ & $4.84 \mathrm{E}-5$ & $>1.00 \mathrm{E}-4$ \\
\hline BT-54 & 1.085 & 2.140 & 2.083 & 1.647 & 1.617 & 1.21 & 0.7 & 95 & 53 & 50 & 12 & -29 & 1.03 & & $>1.00 \mathrm{E}-4$ \\
\hline T-47! & 1.153 & 2.518 & 2.315 & 1.612 & 1.730 & 1.678 & 1.064 & 85 & 34 & 42 & 38 & -8 & $4.81 \mathrm{E}-8$ & $6.79 \mathrm{E}-5$ & $>1.00 \mathrm{E}-4$ \\
\hline MDA-MB-468 & 0.815 & 1.716 & 1.635 & 1.062 & 0.902 & 0.895 & 0.591 & 91 & 27 & 10 & 9 & -28 & $4.42 \mathrm{E}-8$ & $1.75 \mathrm{E}-5$ & $>1.00 \mathrm{E}-4$ \\
\hline
\end{tabular}


In-Vitro Testing Results

\begin{tabular}{|l|l|l|l|}
\hline NSC : D - 826091 / 1 & Experiment ID : 2011NS60 & Test Type : 08 & Units : Molar \\
\hline Report Date : March 24, 2021 & Test Date : November 02, 2020 & QNS : & MC: \\
\hline COMI : KLB2-41 & Stain Reagent : SRB Dual-Pass Related & SSPL : 1C1X & \\
\hline
\end{tabular}

Log10 Concentration

\begin{tabular}{|c|c|c|c|c|c|c|c|c|c|c|c|c|c|c|c|}
\hline \multirow[b]{2}{*}{ Panel/Cell Line } & \multicolumn{3}{|l|}{ Time } & \multicolumn{5}{|c|}{ Mean Optical Densities } & \multicolumn{4}{|c|}{ Percent Growth } & \multirow[b]{2}{*}{ GI50 } & \multirow[b]{2}{*}{ TGI } & \multirow[b]{2}{*}{ LC50 } \\
\hline & Zero & Ctrl & -8.0 & -7.0 & -6.0 & -5.0 & -4.0 & -8.0 & -7.0 & -6.0 & -5.0 & -4.0 & & & \\
\hline $\begin{array}{l}\text { Leukemia } \\
\text { CCRF-CEM } \\
\text { HL-60(TB) } \\
\text { K-562 } \\
\text { MOLT-4 } \\
\text { RPMI-8226 } \\
\text { SR }\end{array}$ & $\begin{array}{l}0.448 \\
0.657 \\
0.127 \\
0.482 \\
0.715 \\
0.221\end{array}$ & $\begin{array}{l}2.433 \\
2.862 \\
1.133 \\
2.592 \\
2.645 \\
0.982\end{array}$ & $\begin{array}{l}2.554 \\
2.654 \\
1.104 \\
2.659 \\
2.728 \\
0.927\end{array}$ & $\begin{array}{l}2.509 \\
2.753 \\
1.074 \\
2.655 \\
2.698 \\
0.838\end{array}$ & $\begin{array}{l}1.376 \\
1.256 \\
0.400 \\
1.669 \\
2.219 \\
0.367\end{array}$ & $\begin{array}{l}0.712 \\
0.536 \\
0.189 \\
0.746 \\
0.924 \\
0.313\end{array}$ & $\begin{array}{l}0.344 \\
0.327 \\
0.117 \\
0.371 \\
0.580 \\
0.140\end{array}$ & $\begin{array}{r}106 \\
91 \\
97 \\
103 \\
104 \\
93\end{array}$ & $\begin{array}{r}104 \\
95 \\
94 \\
103 \\
103 \\
81\end{array}$ & $\begin{array}{l}47 \\
27 \\
27 \\
56 \\
78 \\
19\end{array}$ & $\begin{array}{r}13 \\
-18 \\
6 \\
13 \\
11 \\
12\end{array}$ & $\begin{array}{r}-23 \\
-50 \\
-8 \\
-23 \\
-19 \\
-37\end{array}$ & $\begin{array}{l}8.77 \mathrm{E}-7 \\
4.61 \mathrm{E}-7 \\
4.55 \mathrm{E}-7 \\
1.39 \mathrm{E}-6 \\
2.61 \mathrm{E}-6 \\
3.17 \mathrm{E}-7\end{array}$ & $\begin{array}{l}2.31 \mathrm{E}-5 \\
3.93 \mathrm{E}-6 \\
2.74 \mathrm{E}-5 \\
2.25 \mathrm{E}-5 \\
2.31 \mathrm{E}-5 \\
1.77 \mathrm{E}-5\end{array}$ & $\begin{array}{r}>1.00 \mathrm{E}-4 \\
\quad 9.78 \mathrm{E}-5 \\
>1.00 \mathrm{E}-4 \\
>1.00 \mathrm{E}-4 \\
>1.00 \mathrm{E}-4 \\
>1.00 \mathrm{E}-4\end{array}$ \\
\hline $\begin{array}{l}\text { Non-Small Cell Lung } \\
\text { A549/ATCC } \\
\text { EKVX } \\
\text { HOP-62 } \\
\text { HOP-92 } \\
\mathrm{NCl}-\mathrm{H} 226 \\
\mathrm{NCl}-\mathrm{H} 23 \\
\mathrm{NCl}-\mathrm{H} 322 \mathrm{M} \\
\mathrm{NCl}-\mathrm{H} 460 \\
\mathrm{NCl}-\mathrm{H} 522\end{array}$ & $\begin{array}{l}\text { Cancer } \\
0.405 \\
0.833 \\
0.686 \\
1.222 \\
0.714 \\
0.583 \\
0.802 \\
0.331 \\
1.044\end{array}$ & $\begin{array}{l}1.992 \\
2.189 \\
2.136 \\
1.482 \\
1.808 \\
2.052 \\
2.214 \\
2.807 \\
2.738\end{array}$ & $\begin{array}{l}1.909 \\
2.128 \\
2.097 \\
1.461 \\
1.762 \\
1.998 \\
2.131 \\
2.829 \\
2.650\end{array}$ & $\begin{array}{l}1.995 \\
2.173 \\
2.094 \\
1.437 \\
1.838 \\
2.082 \\
2.109 \\
2.878 \\
2.568\end{array}$ & $\begin{array}{l}1.359 \\
1.853 \\
1.513 \\
1.304 \\
1.672 \\
1.708 \\
2.034 \\
1.831 \\
1.660\end{array}$ & $\begin{array}{l}0.545 \\
1.102 \\
1.004 \\
1.101 \\
0.986 \\
0.797 \\
1.331 \\
0.350 \\
0.990\end{array}$ & $\begin{array}{l}0.093 \\
0.155 \\
0.225 \\
0.162 \\
0.250 \\
0.085 \\
0.041 \\
0.051 \\
0.223\end{array}$ & $\begin{array}{r}95 \\
95 \\
97 \\
92 \\
96 \\
96 \\
94 \\
101 \\
95\end{array}$ & $\begin{array}{r}100 \\
99 \\
97 \\
83 \\
103 \\
102 \\
93 \\
103 \\
90\end{array}$ & $\begin{array}{l}60 \\
75 \\
57 \\
32 \\
88 \\
77 \\
87 \\
61 \\
36\end{array}$ & $\begin{array}{r}9 \\
20 \\
22 \\
-10 \\
25 \\
15 \\
37 \\
1 \\
-5\end{array}$ & $\begin{array}{l}-77 \\
-81 \\
-67 \\
-87 \\
-65 \\
-85 \\
-95 \\
-85 \\
-79\end{array}$ & $\begin{array}{l}1.57 \mathrm{E}-6 \\
2.85 \mathrm{E}-6 \\
1.59 \mathrm{E}-6 \\
4.34 \mathrm{E}-7 \\
3.97 \mathrm{E}-6 \\
2.68 \mathrm{E}-6 \\
5.60 \mathrm{E}-6 \\
1.50 \mathrm{E}-6 \\
5.57 \mathrm{E}-7\end{array}$ & $\begin{array}{l}1.27 \mathrm{E}-5 \\
1.57 \mathrm{E}-5 \\
1.76 \mathrm{E}-5 \\
5.76 \mathrm{E}-6 \\
1.89 \mathrm{E}-5 \\
1.40 \mathrm{E}-5 \\
1.92 \mathrm{E}-5 \\
1.02 \mathrm{E}-5 \\
7.49 \mathrm{E}-6\end{array}$ & $\begin{array}{l}4.83 \mathrm{E}-5 \\
4.89 \mathrm{E}-5 \\
6.41 \mathrm{E}-5 \\
3.32 \mathrm{E}-5 \\
6.81 \mathrm{E}-5 \\
4.42 \mathrm{E}-5 \\
4.58 \mathrm{E}-5 \\
3.93 \mathrm{E}-5 \\
4.07 \mathrm{E}-5\end{array}$ \\
\hline $\begin{array}{l}\text { Colon Cancer } \\
\text { COLO } 205 \\
\text { HCC-2998 } \\
\text { HCT-116 } \\
\text { HCT-15 } \\
\text { HT29 } \\
\text { KM12 } \\
\text { SW-620 }\end{array}$ & $\begin{array}{l}0.541 \\
0.804 \\
0.243 \\
0.302 \\
0.365 \\
0.614 \\
0.266\end{array}$ & $\begin{array}{l}1.955 \\
2.708 \\
2.147 \\
2.271 \\
2.130 \\
2.806 \\
1.976\end{array}$ & $\begin{array}{l}2.029 \\
2.523 \\
2.147 \\
2.198 \\
2.065 \\
2.788 \\
1.913\end{array}$ & $\begin{array}{l}1.974 \\
2.542 \\
2.136 \\
2.244 \\
2.076 \\
2.788 \\
1.934\end{array}$ & $\begin{array}{l}1.718 \\
2.281 \\
1.240 \\
0.992 \\
1.246 \\
1.652 \\
0.934\end{array}$ & $\begin{array}{l}0.743 \\
0.885 \\
0.378 \\
0.463 \\
0.309 \\
0.798 \\
0.489\end{array}$ & $\begin{array}{l}0.218 \\
0.119 \\
0.028 \\
0.049 \\
0.148 \\
0.118 \\
0.029\end{array}$ & $\begin{array}{r}105 \\
90 \\
100 \\
96 \\
96 \\
99 \\
96\end{array}$ & $\begin{array}{r}101 \\
91 \\
99 \\
99 \\
97 \\
99 \\
98\end{array}$ & $\begin{array}{l}83 \\
78 \\
52 \\
35 \\
50 \\
47 \\
39\end{array}$ & $\begin{array}{r}14 \\
4 \\
7 \\
8 \\
-15 \\
8 \\
13\end{array}$ & $\begin{array}{l}-60 \\
-85 \\
-88 \\
-84 \\
-59 \\
-81 \\
-89\end{array}$ & $\begin{array}{l}3.04 \mathrm{E}-6 \\
2.37 \mathrm{E}-6 \\
1.13 \mathrm{E}-6 \\
5.82 \mathrm{E}-7 \\
9.94 \mathrm{E}-7 \\
8.88 \mathrm{E}-7 \\
6.50 \mathrm{E}-7\end{array}$ & $\begin{array}{l}1.56 \mathrm{E}-5 \\
1.11 \mathrm{E}-5 \\
1.19 \mathrm{E}-5 \\
1.23 \mathrm{E}-5 \\
5.80 \mathrm{E}-6 \\
1.24 \mathrm{E}-5 \\
1.34 \mathrm{E}-5\end{array}$ & $\begin{array}{l}7.39 \mathrm{E}-5 \\
4.04 \mathrm{E}-5 \\
3.96 \mathrm{E}-5 \\
4.28 \mathrm{E}-5 \\
6.10 \mathrm{E}-5 \\
4.52 \mathrm{E}-5 \\
4.14 \mathrm{E}-5\end{array}$ \\
\hline $\begin{array}{l}\text { CNS Cancer } \\
\text { SF-268 } \\
\text { SF-295 } \\
\text { SF-539 } \\
\text { SNB-19 } \\
\text { SNB-75 } \\
\text { U251 }\end{array}$ & $\begin{array}{l}1.116 \\
0.392 \\
0.511 \\
0.555 \\
1.652 \\
0.378\end{array}$ & $\begin{array}{l}2.636 \\
2.137 \\
1.795 \\
1.970 \\
2.273 \\
1.809\end{array}$ & $\begin{array}{l}2.560 \\
2.159 \\
1.764 \\
1.880 \\
1.960 \\
1.711\end{array}$ & $\begin{array}{l}2.598 \\
2.192 \\
1.816 \\
1.939 \\
2.022 \\
1.751\end{array}$ & $\begin{array}{l}2.230 \\
0.533 \\
1.256 \\
1.358 \\
1.556 \\
1.225\end{array}$ & $\begin{array}{l}1.521 \\
0.214 \\
0.395 \\
0.940 \\
1.247 \\
0.517\end{array}$ & $\begin{array}{l}0.335 \\
0.022 \\
0.065 \\
0.053 \\
0.158 \\
0.045\end{array}$ & $\begin{array}{r}95 \\
101 \\
98 \\
94 \\
50 \\
93\end{array}$ & $\begin{array}{r}98 \\
103 \\
102 \\
98 \\
60 \\
96\end{array}$ & $\begin{array}{r}73 \\
8 \\
58 \\
57 \\
-6 \\
59\end{array}$ & $\begin{array}{r}27 \\
-46 \\
-23 \\
27 \\
-25 \\
10\end{array}$ & $\begin{array}{l}-70 \\
-95 \\
-87 \\
-91 \\
-90 \\
-88\end{array}$ & $\begin{array}{r}3.15 \mathrm{E}-6 \\
3.62 \mathrm{E}-7 \\
1.26 \mathrm{E}-6 \\
1.69 \mathrm{E}-6 \\
1.53 \mathrm{E}-6\end{array}$ & $\begin{array}{l}1.89 \mathrm{E}-5 \\
1.41 \mathrm{E}-6 \\
5.23 \mathrm{E}-6 \\
1.70 \mathrm{E}-5 \\
8.15 \mathrm{E}-7 \\
1.26 \mathrm{E}-5\end{array}$ & $\begin{array}{l}6.21 \mathrm{E}-5 \\
1.23 \mathrm{E}-5 \\
2.65 \mathrm{E}-5 \\
4.53 \mathrm{E}-5 \\
2.43 \mathrm{E}-5 \\
4.07 \mathrm{E}-5\end{array}$ \\
\hline $\begin{array}{l}\text { Melanoma } \\
\text { LOX IMVI } \\
\text { MALME-3M } \\
\text { M14 } \\
\text { MDA-MB-435 } \\
\text { SK-MEL-2 } \\
\text { SK-MEL-28 } \\
\text { SK-MEL-5 } \\
\text { UACC-257 } \\
\text { UACC-62 }\end{array}$ & $\begin{array}{l}0.281 \\
0.783 \\
0.447 \\
0.585 \\
1.367 \\
0.590 \\
0.955 \\
1.398 \\
0.867\end{array}$ & $\begin{array}{l}1.931 \\
1.820 \\
1.796 \\
2.390 \\
2.885 \\
1.874 \\
3.309 \\
2.922 \\
2.939\end{array}$ & $\begin{array}{l}1.828 \\
1.741 \\
1.749 \\
2.313 \\
2.871 \\
1.844 \\
3.317 \\
2.816 \\
2.775\end{array}$ & $\begin{array}{l}1.961 \\
1.858 \\
1.745 \\
2.235 \\
2.815 \\
1.912 \\
3.337 \\
2.861 \\
2.859\end{array}$ & $\begin{array}{l}1.366 \\
1.324 \\
1.113 \\
0.534 \\
2.185 \\
1.406 \\
2.793 \\
2.486 \\
1.447\end{array}$ & $\begin{array}{l}0.751 \\
1.196 \\
0.554 \\
0.388 \\
1.685 \\
1.056 \\
1.262 \\
1.917 \\
1.104\end{array}$ & $\begin{array}{l}0.037 \\
0.234 \\
0.095 \\
0.038 \\
0.372 \\
0.088 \\
0.018 \\
0.462 \\
0.047\end{array}$ & $\begin{array}{r}94 \\
92 \\
96 \\
96 \\
99 \\
98 \\
100 \\
93 \\
92\end{array}$ & $\begin{array}{r}102 \\
104 \\
96 \\
91 \\
95 \\
103 \\
101 \\
96 \\
96\end{array}$ & $\begin{array}{l}66 \\
52 \\
49 \\
-9 \\
54 \\
64 \\
78 \\
71 \\
28\end{array}$ & $\begin{array}{r}28 \\
40 \\
8 \\
-34 \\
21 \\
36 \\
13 \\
34 \\
11\end{array}$ & $\begin{array}{l}-87 \\
-70 \\
-79 \\
-94 \\
-73 \\
-85 \\
-98 \\
-67 \\
-95\end{array}$ & $\begin{array}{l}2.64 \mathrm{E}-6 \\
1.50 \mathrm{E}-6 \\
9.69 \mathrm{E}-7 \\
2.59 \mathrm{E}-7 \\
1.31 \mathrm{E}-6 \\
3.13 \mathrm{E}-6 \\
2.70 \mathrm{E}-6 \\
3.74 \mathrm{E}-6 \\
4.75 \mathrm{E}-7\end{array}$ & $\begin{array}{l}1.77 \mathrm{E}-5 \\
2.30 \mathrm{E}-5 \\
1.23 \mathrm{E}-5 \\
8.17 \mathrm{E}-7 \\
1.67 \mathrm{E}-5 \\
1.99 \mathrm{E}-5 \\
1.31 \mathrm{E}-5 \\
2.17 \mathrm{E}-5 \\
1.28 \mathrm{E}-5\end{array}$ & $\begin{array}{l}4.79 \mathrm{E}-5 \\
6.55 \mathrm{E}-5 \\
4.65 \mathrm{E}-5 \\
1.87 \mathrm{E}-5 \\
5.71 \mathrm{E}-5 \\
5.14 \mathrm{E}-5 \\
3.69 \mathrm{E}-5 \\
6.79 \mathrm{E}-5 \\
3.80 \mathrm{E}-5\end{array}$ \\
\hline $\begin{array}{l}\text { Ovarian Cancer } \\
\text { IGROV1 } \\
\text { OVCAR-3 } \\
\text { OVCAR-4 } \\
\text { OVCAR-5 } \\
\text { OVCAR-8 } \\
\text { NCI/ADR-RES } \\
\text { SK-OV-3 }\end{array}$ & $\begin{array}{l}0.484 \\
0.568 \\
0.796 \\
0.430 \\
0.507 \\
0.407 \\
1.283\end{array}$ & $\begin{array}{l}1.923 \\
1.790 \\
1.593 \\
1.379 \\
2.285 \\
1.552 \\
2.406\end{array}$ & $\begin{array}{l}1.876 \\
1.835 \\
1.633 \\
1.357 \\
2.270 \\
1.546 \\
2.393\end{array}$ & $\begin{array}{l}1.944 \\
1.824 \\
1.605 \\
1.362 \\
2.251 \\
1.571 \\
2.403\end{array}$ & $\begin{array}{l}1.293 \\
0.761 \\
1.467 \\
1.240 \\
1.971 \\
0.900 \\
2.107\end{array}$ & $\begin{array}{l}0.734 \\
0.463 \\
0.942 \\
0.549 \\
0.795 \\
0.372 \\
1.607\end{array}$ & $\begin{array}{l}0.185 \\
0.023 \\
0.105 \\
0.051 \\
0.144 \\
0.109 \\
0.151\end{array}$ & $\begin{array}{r}97 \\
104 \\
105 \\
98 \\
99 \\
99 \\
99\end{array}$ & $\begin{array}{r}101 \\
103 \\
101 \\
98 \\
98 \\
102 \\
100\end{array}$ & $\begin{array}{l}56 \\
16 \\
84 \\
85 \\
82 \\
43 \\
73\end{array}$ & $\begin{array}{r}17 \\
-18 \\
18 \\
13 \\
16 \\
-9 \\
29\end{array}$ & $\begin{array}{l}-62 \\
-96 \\
-87 \\
-88 \\
-72 \\
-73 \\
-88\end{array}$ & $\begin{array}{l}1.45 \mathrm{E}-6 \\
4.04 \mathrm{E}-7 \\
3.30 \mathrm{E}-6 \\
3.06 \mathrm{E}-6 \\
3.08 \mathrm{E}-6 \\
7.61 \mathrm{E}-7 \\
3.35 \mathrm{E}-6\end{array}$ & $\begin{array}{l}1.66 \mathrm{E}-5 \\
2.89 \mathrm{E}-6 \\
1.49 \mathrm{E}-5 \\
1.33 \mathrm{E}-5 \\
1.53 \mathrm{E}-5 \\
6.82 \mathrm{E}-6 \\
1.76 \mathrm{E}-5\end{array}$ & $\begin{array}{l}7.08 \mathrm{E}-5 \\
2.55 \mathrm{E}-5 \\
4.47 \mathrm{E}-5 \\
4.18 \mathrm{E}-5 \\
5.66 \mathrm{E}-5 \\
4.36 \mathrm{E}-5 \\
4.72 \mathrm{E}-5\end{array}$ \\
\hline $\begin{array}{l}\text { Renal Cancer } \\
786-0 \\
\text { ACHN } \\
\text { CAKI-1 } \\
\text { RXF 393 } \\
\text { SN12C } \\
\text { TK-10 } \\
\text { UO-31 }\end{array}$ & $\begin{array}{l}0.632 \\
0.403 \\
0.490 \\
0.694 \\
0.457 \\
0.969 \\
0.655\end{array}$ & $\begin{array}{l}2.409 \\
1.694 \\
1.899 \\
1.380 \\
1.741 \\
1.902 \\
2.264\end{array}$ & $\begin{array}{l}2.238 \\
1.735 \\
1.778 \\
1.370 \\
1.650 \\
1.810 \\
2.116\end{array}$ & $\begin{array}{l}2.264 \\
1.733 \\
1.816 \\
1.348 \\
1.723 \\
1.796 \\
2.201\end{array}$ & $\begin{array}{l}1.851 \\
1.333 \\
1.057 \\
1.121 \\
1.479 \\
1.698 \\
1.812\end{array}$ & $\begin{array}{l}1.046 \\
0.742 \\
0.764 \\
0.634 \\
0.721 \\
1.171 \\
0.999\end{array}$ & $\begin{array}{l}0.155 \\
0.040 \\
0.035 \\
0.108 \\
0.038 \\
0.271 \\
0.054\end{array}$ & $\begin{array}{r}90 \\
103 \\
91 \\
99 \\
93 \\
90 \\
91\end{array}$ & $\begin{array}{r}92 \\
103 \\
94 \\
95 \\
99 \\
89 \\
96\end{array}$ & $\begin{array}{l}69 \\
72 \\
40 \\
62 \\
80 \\
78 \\
72\end{array}$ & $\begin{array}{l}23 \\
26 \\
19 \\
-9 \\
21 \\
22 \\
21\end{array}$ & $\begin{array}{l}-75 \\
-90 \\
-93 \\
-85 \\
-92 \\
-72 \\
-92\end{array}$ & $\begin{array}{l}2.57 \mathrm{E}-6 \\
3.03 \mathrm{E}-6 \\
6.58 \mathrm{E}-7 \\
1.49 \mathrm{E}-6 \\
3.17 \mathrm{E}-6 \\
3.15 \mathrm{E}-6 \\
2.71 \mathrm{E}-6\end{array}$ & $\begin{array}{l}1.72 \mathrm{E}-5 \\
1.68 \mathrm{E}-5 \\
1.49 \mathrm{E}-5 \\
7.55 \mathrm{E}-6 \\
1.52 \mathrm{E}-5 \\
1.70 \mathrm{E}-5 \\
1.55 \mathrm{E}-5\end{array}$ & $\begin{array}{l}5.52 \mathrm{E}-5 \\
4.52 \mathrm{E}-5 \\
4.15 \mathrm{E}-5 \\
3.51 \mathrm{E}-5 \\
4.25 \mathrm{E}-5 \\
5.82 \mathrm{E}-5 \\
4.27 \mathrm{E}-5\end{array}$ \\
\hline $\begin{array}{l}\text { Prostate Cancer } \\
\text { PC-3 } \\
\text { DU-145 }\end{array}$ & $\begin{array}{l}0.533 \\
0.300\end{array}$ & $\begin{array}{l}1.906 \\
1.329\end{array}$ & $\begin{array}{l}1.834 \\
1.369\end{array}$ & $\begin{array}{l}1.857 \\
1.353\end{array}$ & $\begin{array}{l}1.250 \\
1.133\end{array}$ & $\begin{array}{l}0.624 \\
0.374\end{array}$ & $\begin{array}{l}0.040 \\
0.019\end{array}$ & $\begin{array}{r}95 \\
104\end{array}$ & $\begin{array}{r}96 \\
102\end{array}$ & $\begin{array}{l}52 \\
81\end{array}$ & $\begin{array}{l}7 \\
7\end{array}$ & $\begin{array}{l}-92 \\
-94\end{array}$ & $\begin{array}{l}1.12 \mathrm{E}-6 \\
2.63 \mathrm{E}-6\end{array}$ & $\begin{array}{l}1.17 \mathrm{E}-5 \\
1.18 \mathrm{E}-5\end{array}$ & $\begin{array}{l}3.73 E-5 \\
3.69 E-5\end{array}$ \\
\hline $\begin{array}{l}\text { Breast Cancer } \\
\text { MCF7 } \\
\text { MDA-MB-231/ATCC } \\
\text { HS 578T } \\
\text { BT-549 } \\
\text { T-47D } \\
\text { MDA-MB-468 }\end{array}$ & $\begin{array}{r}0.305 \\
0.563 \\
1.432 \\
1.107 \\
1.309 \\
0.786\end{array}$ & $\begin{array}{l}1.949 \\
1.464 \\
1.963 \\
2.107 \\
2.657 \\
1.663\end{array}$ & $\begin{array}{l}1.713 \\
1.457 \\
1.866 \\
1.997 \\
2.515 \\
1.567\end{array}$ & $\begin{array}{l}1.760 \\
1.483 \\
1.938 \\
2.109 \\
2.533 \\
1.556\end{array}$ & $\begin{array}{l}0.798 \\
1.300 \\
1.550 \\
1.652 \\
2.096 \\
0.889\end{array}$ & $\begin{array}{l}0.422 \\
0.821 \\
1.087 \\
1.324 \\
1.477 \\
0.661\end{array}$ & $\begin{array}{l}0.050 \\
0.163 \\
0.429 \\
0.170 \\
0.697 \\
0.072\end{array}$ & $\begin{array}{l}86 \\
99 \\
82 \\
89 \\
89 \\
89\end{array}$ & $\begin{array}{r}88 \\
102 \\
95 \\
100 \\
91 \\
88\end{array}$ & $\begin{array}{l}30 \\
82 \\
22 \\
54 \\
58 \\
12\end{array}$ & $\begin{array}{r}7 \\
29 \\
-24 \\
22 \\
12 \\
-16\end{array}$ & $\begin{array}{l}-84 \\
-71 \\
-70 \\
-85 \\
-47 \\
-91\end{array}$ & $\begin{array}{l}4.54 \mathrm{E}-7 \\
3.96 \mathrm{E}-6 \\
4.16 \mathrm{E}-7 \\
1.37 \mathrm{E}-6 \\
1.52 \mathrm{E}-6 \\
3.14 \mathrm{E}-7\end{array}$ & $\begin{array}{l}1.20 \mathrm{E}-5 \\
1.94 \mathrm{E}-5 \\
3.01 \mathrm{E}-6 \\
1.60 \mathrm{E}-5 \\
1.62 \mathrm{E}-5 \\
2.65 \mathrm{E}-6\end{array}$ & $\begin{array}{r}4.25 \mathrm{E}-5 \\
6.14 \mathrm{E}-5 \\
3.66 \mathrm{E}-5 \\
4.72 \mathrm{E}-5 \\
>1.00 \mathrm{E}-4 \\
2.85 \mathrm{E}-5\end{array}$ \\
\hline
\end{tabular}


In-Vitro Testing Results

\begin{tabular}{|l|l|l|l}
\hline NSC : D - 826498 / 1 & Experiment ID : 2101NS68 & Test Type : 08 & Units : Molar \\
\hline Report Date : March 24, 2021 & Test Date : January 04, 2021 & QNS : & MC : \\
\hline COMI : KLB2-48 & Stain Reagent : SRB Dual-Pass Related & SSPL : 1C1X & \\
\hline
\end{tabular}

Log10 Concentration

\begin{tabular}{|c|c|c|c|c|c|c|c|c|c|c|c|c|c|c|c|}
\hline \multirow[b]{2}{*}{ Panel/Cell Line } & \multirow{2}{*}{$\begin{array}{l}\text { Time } \\
\text { Zero }\end{array}$} & \multirow[b]{2}{*}{ Ctrl } & \multicolumn{5}{|c|}{ Mean Optical Densities } & \multicolumn{5}{|c|}{ Percent Growth } & \multirow[b]{2}{*}{ GI50 } & \multirow[b]{2}{*}{ TGI } & \\
\hline & & & -8.0 & -7.0 & -6.0 & -5.0 & -4.0 & -8.0 & -7.0 & -6.0 & -5.0 & -4.0 & & & LC50 \\
\hline $\begin{array}{l}\text { Leukemia } \\
\text { CCRF-CEM }\end{array}$ & 0.488 & 1810 & 1813 & 1.453 & 0.591 & 0.556 & 0.299 & 100 & 73 & 8 & & -39 & & & \\
\hline $\begin{array}{l}\text { CLRF-CEMI } \\
\text { HL-60(TB) }\end{array}$ & $\begin{array}{l}.488 \\
0.693\end{array}$ & $\begin{array}{l}1.810 \\
2.262\end{array}$ & $\begin{array}{l}1.873 \\
2.223\end{array}$ & $\begin{array}{l}1.453 \\
1.555\end{array}$ & $\begin{array}{l}0.591 \\
0.567\end{array}$ & $\begin{array}{l}0.556 \\
0.393\end{array}$ & $\begin{array}{l}0.299 \\
0.268\end{array}$ & $\begin{array}{r}100 \\
97\end{array}$ & 55 & $\begin{array}{r}8 \\
-18\end{array}$ & $\begin{array}{c}5 \\
-43\end{array}$ & $\begin{array}{l}-39 \\
-61\end{array}$ & $\begin{array}{l}2.25 \mathrm{E}-1 \\
1.17 \mathrm{E}-7\end{array}$ & $\begin{array}{l}1.37 \mathrm{E}-5 \\
5.63 \mathrm{E}-7\end{array}$ & $\begin{array}{l}> \\
7.00 \mathrm{E}-4 \\
2.33 \mathrm{E}-5\end{array}$ \\
\hline K-562 & 0.282 & 2.217 & 2.129 & 0.824 & 0.419 & 0.328 & 0.133 & 95 & 28 & 7 & 2 & -53 & $4.72 \mathrm{E}-8$ & 1.10E-5 & $8.88 \mathrm{E}-5$ \\
\hline MOLT-4 & 0.721 & 2.386 & 2.432 & 2.298 & 1.105 & 0.797 & 0.435 & 103 & 95 & 23 & 5 & -40 & 4.20E-7 & $1.27 \mathrm{E}-5$ & $>1.00 \mathrm{E}-4$ \\
\hline RPMI-8226 & 1.118 & 2.333 & 2.302 & 2.008 & 1.128 & 0.889 & 0.804 & 97 & 73 & 1 & -21 & -28 & $2.09 \mathrm{E}-7$ & $1.09 \mathrm{E}-6$ & $>1.00 \mathrm{E}-4$ \\
\hline SR & 0.683 & 2.558 & 2.265 & 1.031 & 0.887 & 0.629 & 0.338 & 84 & 19 & 11 & -8 & -51 & 3.33E-8 & $3.79 \mathrm{E}-6$ & $9.73 \mathrm{E}-5$ \\
\hline Non-Small Cell Lung & Cancer & & & & & & & & & & & & & & \\
\hline A549/ATCC & 0.420 & 2.474 & 2.448 & 1.992 & 1.128 & 0.692 & 0.200 & 99 & 77 & 34 & 13 & -52 & $4.28 \mathrm{E}-7$ & $1.59 \mathrm{E}-5$ & $9.20 \mathrm{E}-5$ \\
\hline EKVX & 0.568 & 1.555 & 1.533 & 1.424 & 0.961 & 0.743 & 0.249 & 98 & 87 & 40 & 18 & -56 & 6.07E-7 & $1.74 \mathrm{E}-5$ & $8.25 \mathrm{E}-5$ \\
\hline HOP-62 & 0.895 & 2.733 & 2.546 & 2.226 & 1.588 & 1.350 & 0.099 & 90 & 72 & 38 & 25 & -89 & $4.42 \mathrm{E}-7$ & 1.65E-5 & $4.55 \mathrm{E}-5$ \\
\hline HOP-92 & 1.491 & 2.113 & 1.972 & 1.875 & 1.750 & 1.281 & 0.278 & 77 & 62 & 42 & -14 & -81 & 3.80E-7 & $5.58 \mathrm{E}-6$ & $3.42 \mathrm{E}-5$ \\
\hline $\mathrm{NCl}-\mathrm{H} 226$ & 1.047 & 2.263 & 2.131 & 2.097 & 1.450 & 1.083 & 0.235 & 89 & 86 & 33 & 3 & -78 & $4.82 \mathrm{E}-7$ & $1.09 \mathrm{E}-5$ & $4.55 \mathrm{E}-5$ \\
\hline $\mathrm{NCl}-\mathrm{H} 23$ & 0.661 & 1.875 & 1.823 & 1.689 & 1.101 & 0.817 & 0.140 & 96 & 85 & 36 & 13 & -79 & 5.19E-7 & $1.38 \mathrm{E}-5$ & 4.84E-5 \\
\hline $\mathrm{NCl}-\mathrm{H} 322 \mathrm{M}$ & 0.767 & 2.239 & 2.268 & 2.181 & 1.417 & 1.469 & 0.190 & 102 & 96 & 44 & 48 & -75 & 7.70E-7 & $2.44 \mathrm{E}-5$ & $6.23 \mathrm{E}-5$ \\
\hline $\mathrm{NCl}-\mathrm{H} 460$ & 0.296 & 2.670 & 2.747 & 1.945 & 0.507 & 0.285 & 0.059 & 103 & 69 & 9 & -4 & -80 & 2.10E-7 & $5.07 \mathrm{E}-6$ & $4.04 \mathrm{E}-5$ \\
\hline Colon Cancer & & & & & & & & & & & & & & & \\
\hline COLO 205 & 0.637 & 2.665 & 2.632 & 2.320 & 0.751 & 0.411 & 0.131 & 98 & 83 & 6 & -36 & -79 & 2.67E-7 & $1.37 \mathrm{E}-6$ & $2.13 \mathrm{E}-5$ \\
\hline HCC-2998 & 0.633 & 2.020 & 2.017 & 1.772 & 1.264 & 0.751 & 0.056 & 100 & 82 & 46 & 9 & -91 & $7.54 \mathrm{E}-7$ & $1.22 \mathrm{E}-5$ & $3.86 \mathrm{E}-5$ \\
\hline HCT-116 & 0.335 & 2.608 & 2.495 & 1.927 & 0.943 & 0.412 & 0.023 & 95 & 70 & 27 & 3 & -93 & $2.91 \mathrm{E}-7$ & $1.08 \mathrm{E}-5$ & 3.57E-5 \\
\hline HCT-15 & 0.296 & 1.827 & 1.805 & 1.106 & 0.722 & 0.428 & 0.056 & 99 & 53 & 28 & 9 & -81 & $1.30 \mathrm{E}-7$ & $1.25 \mathrm{E}-5$ & $4.49 \mathrm{E}-5$ \\
\hline HT29 & 0.442 & 2.482 & 2.492 & 2.081 & 0.674 & 0.487 & 0.111 & 100 & 80 & 11 & 2 & -75 & $2.75 \mathrm{E}-7$ & 1.07E-5 & $4.74 \mathrm{E}-5$ \\
\hline KM12 & 0.854 & 3.332 & 3.307 & 2.503 & 1.592 & 1.005 & 0.301 & 99 & 67 & 30 & 6 & -65 & $2.82 \mathrm{E}-7$ & $1.22 \mathrm{E}-5$ & $6.19 \mathrm{E}-5$ \\
\hline SW-620 & 0.321 & 1.896 & 1.926 & 1.231 & 0.562 & 0.637 & 0.047 & 102 & 58 & 15 & 20 & -85 & $1.52 \mathrm{E}-7$ & $1.55 \mathrm{E}-5$ & 4.62E-5 \\
\hline CNS Cancer & & & & & & & & & & & & & & & \\
\hline SF-268 & 0.964 & 2.650 & 2.535 & 2.381 & 1.962 & 1.236 & 0.244 & 93 & 84 & 59 & 16 & -75 & 1.63E-6 & 1.50E-5 & $5.34 \mathrm{E}-5$ \\
\hline SF-295 & 0.980 & 3.238 & 3.122 & 2.286 & 1.451 & 1.013 & 0.057 & 95 & 58 & 21 & 1 & -94 & 1.63E-7 & $1.04 \mathrm{E}-5$ & $3.45 E-5$ \\
\hline SF-539 & 1.014 & 2.955 & 2.822 & 2.588 & 1.026 & 0.812 & 0.097 & 93 & 81 & 1 & -20 & -90 & $2.43 \mathrm{E}-7$ & $1.07 \mathrm{E}-6$ & 2.67E-5 \\
\hline SNB-19 & 0.546 & 1.885 & 1.834 & 1.598 & 0.931 & 0.792 & 0.069 & 96 & 79 & 29 & 18 & -87 & 3.75E-7 & 1.49E-5 & $4.43 E-5$ \\
\hline SNB-75 & 1.164 & 2.152 & 1.901 & 1.667 & 1.075 & 1.137 & 0.240 & 75 & 51 & -8 & -2 & -79 & 1.03E-7 & 7.40E-7 & $4.15 \mathrm{E}-5$ \\
\hline U251 & 0.429 & 2.079 & 1.969 & 1.886 & 0.895 & 0.717 & 0.070 & 93 & 88 & 28 & 17 & -84 & 4.34E-7 & 1.49E-5 & $4.64 \mathrm{E}-5$ \\
\hline Melanoma & & & & & & & & & & & & & & & \\
\hline LOX IMVI & 0.305 & 2.001 & 1.875 & 1.418 & 0.886 & 0.344 & 0.047 & 93 & 66 & 34 & 2 & -85 & $3.15 \mathrm{E}-7$ & $1.06 \mathrm{E}-5$ & 3.99E-5 \\
\hline MALME-3M & 0.832 & 2.336 & 2.248 & 2.013 & 1.576 & 1.453 & 0.252 & 94 & 79 & 49 & 41 & -70 & $9.60 \mathrm{E}-7$ & $2.35 \mathrm{E}-5$ & $6.64 \mathrm{E}-5$ \\
\hline M14 & 0.545 & 1.972 & 1.933 & 1.557 & 0.811 & 0.777 & 0.113 & 97 & 71 & 19 & 16 & -79 & $2.51 \mathrm{E}-7$ & $1.48 \mathrm{E}-5$ & $4.94 \mathrm{E}-5$ \\
\hline MDA-MB-435 & 0.575 & 2.672 & 2.588 & 0.810 & 0.434 & 0.614 & 0.055 & 96 & 11 & -25 & 2 & -91 & 3.49E-8 & & 3.64E-5 \\
\hline SK-MEL-2 & 1.298 & 2.440 & 2.379 & 2.108 & 1.893 & 1.607 & 0.270 & 95 & 71 & 52 & 27 & -79 & $1.21 \mathrm{E}-6$ & $1.80 \mathrm{E}-5$ & $5.31 \mathrm{E}-5$ \\
\hline SK-MEL-28 & 0.553 & 1.760 & 1.705 & 1.339 & 1.100 & 0.978 & 0.022 & 95 & 65 & 45 & 35 & -96 & $5.82 \mathrm{E}-7$ & $1.85 \mathrm{E}-5$ & $4.45 \mathrm{E}-5$ \\
\hline SK-MEL-5 & 1.089 & 3.216 & 3.187 & 2.665 & 1.628 & 0.921 & 0.020 & 99 & 74 & 25 & -15 & -98 & $3.12 \mathrm{E}-7$ & $4.18 \mathrm{E}-6$ & 2.62E-5 \\
\hline UACC-257 & 1.013 & 2.536 & 2.413 & 2.291 & 2.060 & 1.375 & 0.414 & 92 & 84 & 69 & 24 & -59 & $2.61 \mathrm{E}-6$ & 1.93E-5 & 7.76E-5 \\
\hline UACC-62 & 0.918 & 2.567 & 2.501 & 1.863 & 1.460 & 1.061 & 0.035 & 96 & 57 & 33 & 9 & -96 & $1.99 \mathrm{E}-7$ & $1.21 \mathrm{E}-5$ & 3.62E-5 \\
\hline Ovaria & & & & & & & & & & & & & & & \\
\hline IGR & 0.342 & 1.940 & 1.912 & 1.482 & 0.801 & 0.538 & 0.087 & 98 & 71 & 29 & 12 & -75 & 3.17E-7 & $1.38 \mathrm{E}-5$ & $5.20 \mathrm{E}-5$ \\
\hline & 0.626 & 1.948 & 1.928 & 1.039 & 0.624 & 0.564 & 0.032 & 99 & 31 & & -10 & -95 & $5.26 \mathrm{E}-8$ & $9.71 \mathrm{E}>2-2$ & $2.96 \mathrm{E}-5$ \\
\hline OVCA & 0.761 & 2.151 & 2.162 & 1.940 & 1.369 & 0.954 & 0.33 & 101 & 85 & 44 & 14 & -5 & $7.03 \mathrm{E}-7$ & & $8.28 \mathrm{E}-5$ \\
\hline & 0.491 & 1.415 & 1378 & 1310 & 0802 & 0671 & 0.03 & 96 & 89 & 34 & 19 & 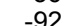 & & & F \\
\hline OVC & 0.738 & 3.029 & 3.003 & 2.905 & 1.766 & 1.206 & 0.311 & 99 & 95 & 45 & 20 & -5 & & & $\begin{array}{l}\text { 4. } \\
794 F-5 \\
7945\end{array}$ \\
\hline NCI/AD & 0.514 & 1.79 & 1.770 & 1.201 & 0.627 & 0.505 & 0.10 & 98 & 54 & 9 & -2 & -8 & $1.20 \mathrm{E}-7$ & & $4.12 \mathrm{E}-5$ \\
\hline SK-OV-3 & 1.344 & 2.565 & 2.482 & 2.428 & 1.886 & 1.739 & 0.456 & 93 & 89 & 44 & 32 & -66 & 7.47E-7 & $2.13 \mathrm{E}-5$ & $6.86 \mathrm{E}-5$ \\
\hline Renal Cancer & & & & & & & & & & & & & & & \\
\hline & 0.678 & 2.634 & 2.541 & 2.444 & 1.359 & 0.929 & 0.164 & 95 & 90 & 35 & 13 & -76 & $5.32 \mathrm{E}-7$ & $1.40 \mathrm{E}-5$ & $5.11 \mathrm{E}-5$ \\
\hline & 1.770 & 2.428 & 2.144 & 2.028 & 1.550 & 1.430 & 0.03 & 57 & 39 & -12 & -19 & -98 & & $5.75 \mathrm{E}-7$ & $2.46 \mathrm{E}-5$ \\
\hline AC & 0.47 & 2.0 & 2.0 & 1.83 & 1.154 & 0.7 & 0.0 & 100 & 85 & 43 & 15 & -99 & & & 3.70E-5 \\
\hline & 0.9 & 3.0 & 2.8 & 1.8 & 1.607 & 1.12 & 0.1 & 89 & 45 & 32 & 10 & -84 & & & $4.34 \mathrm{E}-5$ \\
\hline & 1.0 & 1.5 & 1.44 & 1.3 & 0.808 & 0.85 & 0.1 & 80 & 64 & -24 & -20 & -8 & 1.44 & & $2.82 \mathrm{E}-5$ \\
\hline SN12C & 1.3 & 3.2 & 3.255 & 3.25 & 2.553 & 1.5 & 0.0 & 98 & 98 & 62 & 13 & -9 & & & 3.83E-5 \\
\hline TK-10 & 1.107 & 2.013 & 1.885 & 1.931 & 1.781 & 1.358 & 0.296 & 86 & 91 & 74 & 28 & -73 & & & $5.88 \mathrm{E}-5$ \\
\hline UO-31 & 0.716 & 2.399 & 2.116 & 2.014 & 1.530 & 1.078 & 0.035 & 83 & 77 & 48 & 22 & -95 & $8.77 \mathrm{E}-7$ & $1.53 \mathrm{E}-5$ & $4.10 \mathrm{E}-5$ \\
\hline Prostate Canc & & & & & & & & & & & & & & & \\
\hline & 0.835 & 2.431 & 2.300 & 2.034 & 1.273 & 0.968 & 0.175 & 92 & 75 & 27 & 8 & -79 & $3.36 \mathrm{E}-7$ & $1.24 \mathrm{E}-5$ & $4.65 \mathrm{E}-5$ \\
\hline DU-145 & 0.453 & 1.805 & 1.857 & 1.673 & 0.635 & 0.596 & 0.137 & 104 & 90 & 13 & 11 & -70 & $3.34 \mathrm{E}-7$ & $1.35 \mathrm{E}-5$ & $5.66 \mathrm{E}-5$ \\
\hline Breast Cancer & & & & & & & & & & & & & & & \\
\hline MCF7 & 0.480 & 2.262 & & 1.267 & 0.876 & 0.63 & 0.29 & 92 & 44 & 22 & 9 & & & & \\
\hline MDA-MB-2 & 0.685 & & 1.38 & 1.403 & 0.903 & 0.70 & 0.1 & 95 & 97 & 29 & 3 & -84 & & & 4.04E-5 \\
\hline HS 578T & 0.817 & 1.750 & 1.667 & 1.514 & 1.001 & 0.893 & 0.437 & 91 & 75 & 20 & 8 & -47 & $2.81 \mathrm{E}-7$ & 1.41 & $>1.00 \mathrm{E}-4$ \\
\hline BT-549 & 1.257 & 2.254 & 2.105 & 1.956 & 1.949 & 1.419 & 0.118 & 85 & 70 & 69 & 16 & -91 & $2.32 \mathrm{E}-6$ & 1.42E-5 & 4.17E-5 \\
\hline T-47D & 1.008 & 2.584 & 2.360 & 1.987 & 1.568 & 1.480 & 0.507 & 86 & 62 & 36 & 30 & -50 & $2.86 \mathrm{E}-7$ & $2.37 \mathrm{E}-5$ & $>1.00 \mathrm{E}-4$ \\
\hline MDA-MB-468 & 0.703 & 1.919 & 1.843 & 1.074 & 0.690 & 0.697 & 0.104 & 94 & 30 & -2 & & -85 & $4.91 \mathrm{E}-8$ & $8.72 \mathrm{E}-7$ & 3.82E-5 \\
\hline
\end{tabular}


In-Vitro Testing Results

\begin{tabular}{|l|l|l|l}
\hline NSC : D - 826499 / 1 & Experiment ID : 2101NS68 & Test Type : 08 & Units : Molar \\
\hline Report Date : March 24, 2021 & Test Date : January 04, 2021 & QNS : & MC : \\
\hline COMI : KLB2-49 & Stain Reagent : SRB Dual-Pass Related & SSPL : 1C1X & \\
\hline
\end{tabular}

Log10 Concentration

\begin{tabular}{|c|c|c|c|c|c|c|c|c|c|c|c|c|c|c|c|}
\hline \multirow[b]{2}{*}{ Panel/Cell Line } & \multirow{2}{*}{$\begin{array}{l}\text { Time } \\
\text { Zero }\end{array}$} & \multirow[b]{2}{*}{ Ctrl } & \multicolumn{5}{|c|}{ Mean Optical Densities } & \multicolumn{5}{|c|}{ Percent Growth } & \multirow[b]{2}{*}{ GI50 } & \multirow[b]{2}{*}{ TGI } & \\
\hline & & & -8.0 & -7.0 & -6.0 & -5.0 & -4.0 & -8.0 & -7.0 & -6.0 & -5.0 & -4.0 & & & LC50 \\
\hline $\begin{array}{l}\text { Leukemia } \\
\text { CCRF-CEM }\end{array}$ & 0.488 & 1810 & 1.693 & 1349 & 0.639 & 0.590 & 0.255 & 91 & 65 & 11 & & -48 & & & \\
\hline $\begin{array}{l}\text { CLRF-CEMI } \\
\text { HL-60(TB) }\end{array}$ & $\begin{array}{l}.488 \\
0.693\end{array}$ & $\begin{array}{l}1.810 \\
2.262\end{array}$ & $\begin{array}{l}7.693 \\
2.192\end{array}$ & $\begin{array}{l}1.349 \\
1.277\end{array}$ & $\begin{array}{l}0.639 \\
0.521\end{array}$ & $\begin{array}{l}.0590 \\
0.390\end{array}$ & $\begin{array}{l}0.255 \\
0.284\end{array}$ & $\begin{array}{l}91 \\
96\end{array}$ & $\begin{array}{l}65 \\
37\end{array}$ & $\begin{array}{l}11 \\
-25\end{array}$ & $\begin{array}{r}8 \\
-44\end{array}$ & $\begin{array}{l}-48 \\
-59\end{array}$ & $\begin{array}{l}1.91 \mathrm{E}-1 \\
6.03 \mathrm{E}-8\end{array}$ & $\begin{array}{l}1.38 \mathrm{E}-5 \\
3.97 \mathrm{E}-7\end{array}$ & $\begin{array}{l}>7.00-4 \\
2.54 \mathrm{E}-5\end{array}$ \\
\hline K-562 & 0.282 & 2.217 & 2.188 & 0.811 & 0.464 & 0.338 & 0.127 & 98 & 27 & 9 & 3 & -55 & $4.80 \mathrm{E}-8$ & 1.12E-5 & $8.21 \mathrm{E}-5$ \\
\hline MOLT-4 & 0.721 & 2.386 & 2.411 & 2.132 & 1.120 & 0.829 & 0.466 & 102 & 85 & 24 & 6 & -35 & 3.73E-7 & 1.43E-5 & $>1.00 \mathrm{E}-4$ \\
\hline RPMI-8226 & 1.118 & 2.333 & 2.417 & 1.779 & 1.176 & 0.862 & 0.783 & 107 & 54 & 5 & -23 & -30 & $1.22 \mathrm{E}-7$ & $1.48 \mathrm{E}-6$ & $>1.00 \mathrm{E}-4$ \\
\hline SR & 0.683 & 2.558 & 2.286 & 0.972 & 0.885 & 0.556 & 0.283 & 86 & 15 & 11 & -19 & -59 & $3.21 \mathrm{E}-8$ & $2.32 \mathrm{E}-6$ & $6.08 \mathrm{E}-5$ \\
\hline Non-Small Cell Lung & Cancer & & & & & & & & & & & & & & \\
\hline A549/ATCC & 0.420 & 2.474 & 2.361 & 1.831 & 1.372 & 0.697 & 0.181 & 94 & 69 & 46 & 13 & -57 & $6.86 \mathrm{E}-7$ & $1.55 \mathrm{E}-5$ & $7.95 \mathrm{E}-5$ \\
\hline EKVX & 0.568 & 1.555 & 1.540 & 1.355 & 1.017 & 0.721 & 0.174 & 98 & 80 & 46 & 16 & -69 & 7.39E-7 & $1.52 \mathrm{E}-5$ & $5.90 \mathrm{E}-5$ \\
\hline HOP-62 & 0.895 & 2.733 & 2.555 & 2.155 & 1.632 & 1.324 & 0.145 & 90 & 69 & 40 & 23 & -84 & 4.49E-7 & $1.65 \mathrm{E}-5$ & $4.84 \mathrm{E}-5$ \\
\hline HOP-92 & 1.491 & 2.113 & 2.009 & 1.871 & 1.762 & 1.264 & 0.325 & 83 & 61 & 44 & -15 & -78 & $4.28 \mathrm{E}-7$ & $5.50 \mathrm{E}-6$ & 3.56E-5 \\
\hline $\mathrm{NCl}-\mathrm{H} 226$ & 1.047 & 2.263 & 2.169 & 2.079 & 1.427 & 1.085 & 0.158 & 92 & 85 & 31 & 3 & -85 & 4.47E-7 & $1.09 \mathrm{E}-5$ & $4.01 \mathrm{E}-5$ \\
\hline $\mathrm{NCl}-\mathrm{H} 23$ & 0.661 & 1.875 & 1.842 & 1.562 & 1.179 & 0.762 & 0.162 & 97 & 74 & 43 & 8 & -76 & $5.85 \mathrm{E}-7$ & $1.26 \mathrm{E}-5$ & $4.96 \mathrm{E}-5$ \\
\hline $\mathrm{NCl}-\mathrm{H} 322 \mathrm{M}$ & 0.767 & 2.239 & 2.211 & 2.082 & 1.505 & 1.447 & 0.143 & 98 & 89 & 50 & 46 & -81 & $1.08 \mathrm{E}-6$ & $2.30 \mathrm{E}-5$ & $5.67 \mathrm{E}-5$ \\
\hline $\mathrm{NCl}-\mathrm{H} 460$ & 0.296 & 2.670 & 2.737 & 1.700 & 0.598 & 0.248 & 0.038 & 103 & 59 & 13 & -16 & -87 & $1.57 \mathrm{E}-7$ & $2.75 \mathrm{E}-6$ & $2.99 \mathrm{E}-5$ \\
\hline Colon Cancer & & & & & & & & & & & & & & & \\
\hline COLO 205 & 0.637 & 2.665 & 2.644 & 2.062 & 0.955 & 0.425 & 0.139 & 99 & 70 & 16 & -33 & -78 & $2.35 \mathrm{E}-7$ & $2.09 \mathrm{E}-6$ & $2.35 \mathrm{E}-5$ \\
\hline HCC-2998 & 0.633 & 2.020 & 2.004 & 1.760 & 1.316 & 0.709 & 0.061 & 99 & 81 & 49 & 5 & -90 & $9.48 \mathrm{E}-7$ & $1.14 \mathrm{E}-5$ & $3.79 \mathrm{E}-5$ \\
\hline HCT-116 & 0.335 & 2.608 & 2.551 & 1.674 & 0.968 & 0.402 & 0.034 & 98 & 59 & 28 & 3 & -90 & $1.93 \mathrm{E}-7$ & $1.08 \mathrm{E}-5$ & $3.71 \mathrm{E}-5$ \\
\hline HCT-15 & 0.296 & 1.827 & 1.866 & 1.014 & 0.743 & 0.411 & 0.052 & 102 & 47 & 29 & 7 & -82 & $8.78 \mathrm{E}-8$ & $1.21 \mathrm{E}-5$ & $4.36 \mathrm{E}-5$ \\
\hline HT29 & 0.442 & 2.482 & 2.424 & 1.582 & 0.693 & 0.512 & 0.153 & 97 & 56 & 12 & 3 & -65 & $1.36 \mathrm{E}-7$ & $1.12 \mathrm{E}-5$ & $5.96 \mathrm{E}-5$ \\
\hline KM12 & 0.854 & 3.332 & 3.299 & 2.242 & 1.791 & 1.037 & 0.124 & 99 & 56 & 38 & 7 & -85 & $2.14 \mathrm{E}-7$ & $1.20 \mathrm{E}-5$ & $4.15 \mathrm{E}-5$ \\
\hline SW-620 & 0.321 & 1.896 & 1.765 & 1.144 & 0.563 & 0.597 & 0.037 & 92 & 52 & 15 & 18 & -88 & $1.15 \mathrm{E}-7$ & 1.46E-5 & $4.34 \mathrm{E}-5$ \\
\hline CNS Cancer & & & & & & & & & & & & & & & \\
\hline SF-268 & 0.964 & 2.650 & 2.638 & 2.457 & 2.052 & 1.204 & 0.254 & 99 & 89 & 65 & 14 & -74 & 1.94E-6 & 1.45E-5 & $5.38 \mathrm{E}-5$ \\
\hline SF-295 & 0.980 & 3.238 & 3.133 & 2.270 & 1.484 & 0.810 & 0.029 & 95 & 57 & 22 & -17 & -97 & 1.60E-7 & 3.65E-6 & $2.57 \mathrm{E}-5$ \\
\hline SF-539 & 1.014 & 2.955 & 2.885 & 2.525 & 1.159 & 0.775 & 0.101 & 96 & 78 & 7 & -24 & -90 & $2.49 \mathrm{E}-7$ & $1.74 \mathrm{E}-6$ & $2.50 \mathrm{E}-5$ \\
\hline SNB-19 & 0.546 & 1.885 & 1.839 & 1.610 & 0.993 & 0.785 & 0.044 & 97 & 79 & 33 & 18 & -92 & 4.36E-7 & 1.45E-5 & 4.15E-5 \\
\hline SNB-75 & 1.164 & 2.152 & 2.022 & 1.756 & 1.116 & 1.037 & 0.148 & 87 & 60 & -4 & -11 & -87 & 1.43E-7 & $8.61 \mathrm{E}-7$ & 3.25E-5 \\
\hline U251 & 0.429 & 2.079 & 1.986 & 1.776 & 1.041 & 0.750 & 0.087 & 94 & 82 & 37 & 19 & -80 & $5.13 \mathrm{E}-7$ & $1.57 \mathrm{E}-5$ & $5.02 E-5$ \\
\hline Melanoma & & & & & & & & & & & & & & & \\
\hline LOX IMVI & 0.305 & 2.001 & 1.940 & 1.380 & 0.981 & 0.537 & 0.049 & 96 & 63 & 40 & 14 & -84 & $3.71 \mathrm{E}-7$ & $1.38 \mathrm{E}-5$ & $4.48 \mathrm{E}-5$ \\
\hline MALME-3M & 0.832 & 2.336 & 2.339 & 1.909 & 1.610 & 1.275 & 0.323 & 100 & 72 & 52 & 29 & -61 & 1.20E-6 & $2.11 \mathrm{E}-5$ & $7.52 \mathrm{E}-5$ \\
\hline M14 & 0.545 & 1.972 & 1.913 & 1.446 & 1.013 & 0.722 & 0.102 & 96 & 63 & 33 & 12 & -81 & $2.71 \mathrm{E}-7$ & $1.36 \mathrm{E}-5$ & 4.63E-5 \\
\hline MDA-MB-435 & 0.575 & 2.672 & 2.498 & 0.806 & 0.527 & 0.671 & 0.037 & 92 & 11 & -8 & 5 & -94 & $3.29 \mathrm{E}-8$ & & $3.59 \mathrm{E}-5$ \\
\hline SK-MEL-2 & 1.298 & 2.440 & 2.436 & 2.087 & 1.869 & 1.475 & 0.315 & 100 & 69 & 50 & 15 & -76 & $9.99 \mathrm{E}-7$ & $1.48 \mathrm{E}-5$ & $5.22 \mathrm{E}-5$ \\
\hline SK-MEL-28 & 0.553 & 1.760 & 1.727 & 1.382 & 1.246 & 0.913 & 0.017 & 97 & 69 & 57 & 30 & -97 & $1.86 \mathrm{E}-6$ & $1.72 \mathrm{E}-5$ & 4.26E-5 \\
\hline SK-MEL-5 & 1.089 & 3.216 & 3.170 & 2.560 & 1.555 & 0.523 & 0.008 & 98 & 69 & 22 & -52 & -99 & $2.54 \mathrm{E}-7$ & $1.98 \mathrm{E}-6$ & $9.40 \mathrm{E}-6$ \\
\hline UACC-257 & 1.013 & 2.536 & 2.380 & 2.224 & 1.998 & 1.227 & 0.347 & 90 & 80 & 65 & 14 & -66 & $1.95 \mathrm{E}-6$ & 1.50E-5 & $6.34 \mathrm{E}-5$ \\
\hline UACC-62 & 0.918 & 2.567 & 2.447 & 1.762 & 1.425 & 0.923 & 0.019 & 93 & 51 & 31 & & -98 & $1.14 \mathrm{E}-7$ & $1.01 \mathrm{E}-5$ & 3.25E-5 \\
\hline Ovaria & & & & & & & & & & & & & & & \\
\hline IGR & 0.342 & 1.940 & 1.839 & 1.387 & 0.886 & 0.572 & 0.104 & 94 & 65 & 34 & 14 & -70 & $3.10 \mathrm{E}-7$ & $1.48 \mathrm{E}-5$ & $5.84 \mathrm{E}-5$ \\
\hline & 0.626 & 1.948 & 1.975 & 0.991 & 0.654 & 0.708 & 0.024 & 102 & 28 & 2 & 6 & -96 & $5.00 \mathrm{E}-8$ & $1.15 E-5$ & $3.54 \mathrm{E}-5$ \\
\hline OVCAI & 0.761 & 2.151 & 2.178 & 1.961 & 1.441 & 0.953 & 0.30 & 102 & 86 & 49 & 14 & -6 & 9.37E-7 & 1.54 & $7.38 \mathrm{E}-5$ \\
\hline & 0.491 & 1.415 & 1388 & 1.434 & 0951 & 0.695 & $0.02 \quad \longrightarrow \quad 2$ & 97 & 102 & 50 & 22 & & & & $415-5$ \\
\hline OVC & 0.738 & 3.029 & 3.028 & 2.826 & 1.897 & 1.138 & 0.30 & 100 & 91 & 51 & 17 & -5 - & & & $758-5$ \\
\hline $\left.\mathrm{NCl} / / t_{2}+2\right)$ & 0.514 & 1.79 & 1.756 & 1.147 & 0.712 & 0.506 & $0.10>-10$ & 97 & 49 & 15 & -2 & -8 & & & $4.18 \mathrm{E}-5$ \\
\hline SK-OV-3 & 1.344 & 2.565 & 2.496 & 2.308 & 1.936 & 1.600 & 0.196 & 94 & 79 & 48 & 21 & -85 & $8.89 \mathrm{E}-7$ & $1.57 \mathrm{E}-5$ & $4.64 \mathrm{E}-5$ \\
\hline Renal Cancer & & & & & & & & & & & & & & & \\
\hline & 0.678 & 2.634 & 2.577 & 2.346 & 1.469 & 0.929 & 0.180 & 97 & 85 & 40 & 13 & -74 & $6.12 \mathrm{E}-7$ & $1.41 \mathrm{E}-5$ & $5.34 \mathrm{E}-5$ \\
\hline & 1.770 & 2.428 & 2.233 & 1.976 & 1.609 & 1.432 & 0.03 & 70 & 31 & -9 & -19 & -98 & & $5.94 \mathrm{E}-7$ & $2.46 \mathrm{E}-5$ \\
\hline AC & 0.474 & 2.067 & 2.030 & 1.8 & 1.140 & 0.669 & -0.0 & 98 & 89 & 42 & 12 & -100 & & & 3. $59 \mathrm{E}-5$ \\
\hline & 0.91 & 3.066 & 2.901 & 1.87 & 1.605 & 1.126 & 0.0 & 92 & 45 & 32 & 10 & -9 & & & 3.95E-5 \\
\hline & 1.0 & 1.5 & 1.470 & 1.34 & 0.867 & 0.887 & 0.1 & 84 & 58 & -19 & -17 & -8 & & & $2.97 \mathrm{E}-5$ \\
\hline SN12C & 1.3 & 3.2 & 3.1 & 3.14 & 2.3 & 1.4 & 0.0 & 95 & 92 & 50 & 4 & -9 & & & 3.49E-5 \\
\hline TK-10 & 1.107 & 2.013 & 1.907 & 1.902 & 1.771 & 1.212 & 0.23 & 88 & 88 & 73 & 12 & $-7 \mathrm{c}$ & & & $4.80 \mathrm{E}-5$ \\
\hline UO-31 & 0.716 & 2.399 & 2.207 & 2.006 & 1.593 & 0.986 & 0.058 & 89 & 77 & 52 & 16 & -92 & $1.14 \mathrm{E}-6$ & $1.41 \mathrm{E}-5$ & $4.09 \mathrm{E}-5$ \\
\hline Prostate Can & & & & & & & & & & & & & & & \\
\hline & 0.835 & 2.431 & 2.395 & 1.990 & 1.366 & 0.929 & 0.181 & 98 & 72 & 33 & 6 & -78 & 3.73E-7 & $1.17 \mathrm{E}-5$ & 4.60E-5 \\
\hline DU-145 & 0.453 & 1.805 & 1.903 & 1.662 & 0.821 & 0.630 & 0.087 & 107 & 89 & 27 & 13 & -81 & 4.30E-7 & $1.38 \mathrm{E}-5$ & $4.70 \mathrm{E}-5$ \\
\hline Breast Cancer & & & & & & & & & & & & & & & \\
\hline MCF7 & 0.480 & & 2.086 & 1.331 & 0.849 & 0.611 & & 90 & 48 & 21 & 7 & -51 & & & \\
\hline MDA-MB-2 & 0.685 & 1.427 & 1.450 & 1.393 & 1.027 & 0.716 & 0.1 & 103 & 95 & 46 & 4 & -82 & & & 4.22E-5 \\
\hline HS 578T & 0.817 & 1.7 & 1.6 & 1.455 & 1.050 & 0.8 & 0.4 & 91 & 68 & 25 & 5 & -51 & 2.65 & $1.24 \mathrm{E}-5$ & $9.63 \mathrm{E}-5$ \\
\hline BT-549 & 1.257 & 2.254 & 2.085 & 1.884 & 1.721 & 1.329 & 0.14 & 83 & 63 & 47 & 7 & -89 & $6.12 \mathrm{E}-7$ & 1.19E-5 & $3.94 \mathrm{E}-5$ \\
\hline T-47D & 1.008 & 2.584 & 2.450 & 1.949 & 1.582 & 1.372 & 0.691 & 92 & 60 & 36 & 23 & -31 & $2.62 \mathrm{E}-7$ & $2.65 \mathrm{E}-5$ & $>1.00 \mathrm{E}-4$ \\
\hline MDA-MB-468 & 0.703 & 1.919 & 1.825 & 0.977 & 0.615 & 0.563 & 0.076 & 92 & 23 & -13 & -20 & -89 & $4.04 \mathrm{E}-8$ & 4.39E-7 & $2.72 \mathrm{E}-5$ \\
\hline
\end{tabular}


In-Vitro Testing Results

\begin{tabular}{|l|l|l|l|}
\hline NSC : D - 826502 / 1 & Experiment ID : 2101NS68 & Test Type : 08 & Units : Molar \\
\hline Report Date : March 24, 2021 & Test Date : January 04, 2021 & QNS : & MC : \\
\hline COMI : KLB2-56a & Stain Reagent : SRB Dual-Pass Related & SSPL : 1C1X & \\
\hline
\end{tabular}

Log10 Concentration

\begin{tabular}{|c|c|c|c|c|c|c|c|c|c|c|c|c|c|c|c|}
\hline \multirow[b]{2}{*}{ Panel/Cell Line } & \multirow{2}{*}{$\begin{array}{l}\text { Time } \\
\text { Zero }\end{array}$} & \multirow[b]{2}{*}{ Ctrl } & \multicolumn{5}{|c|}{ Mean Optical Densities } & \multicolumn{5}{|c|}{ Percent Growth } & \multirow[b]{2}{*}{ G150 } & \multirow[b]{2}{*}{ TGI } & \\
\hline & & & -8.0 & -7.0 & -6.0 & -5.0 & -4.0 & -8.0 & -7.0 & -6.0 & -5.0 & -4.0 & & & LC50 \\
\hline Leukemia & & & & & & & & & & & & & & & \\
\hline $\begin{array}{l}\mathrm{HL}-60(\mathrm{~TB}) \\
\mathrm{K}-562\end{array}$ & 0.693 & 2.404 & 2.306 & 0.528 & 0.456 & 0.457 & 0.353 & 94 & -24 & -34 & -34 & -49 & 2.37E-8 & $6.28 \mathrm{E}-8$ & $>1.00 \mathrm{E}-4$ \\
\hline K-562 & 0.282 & 2.238 & 2.219 & 0.615 & 0.408 & 0.385 & 0.277 & 99 & 17 & 6 & 5 & -2 & 3.96E-8 & 5.36E-5 & $>1.00 \mathrm{E}-4$ \\
\hline MOLT-4 & 0.721 & 2.557 & 2.577 & 1.661 & 0.979 & 0.901 & 0.610 & 101 & 51 & 14 & 10 & -15 & 1.08E-7 & $2.44 \mathrm{E}-5$ & $>1.00 \mathrm{E}-4$ \\
\hline RPMI-8226 & 1.118 & 1.968 & 1.828 & 0.834 & 0.652 & 1.132 & 0.806 & 84 & -25 & -42 & 2 & -28 & 2.03E-8 & & $>1.00 \mathrm{E}-4$ \\
\hline SR & 0.683 & 2.797 & 2.538 & 0.954 & 0.871 & 0.727 & 0.498 & 88 & 13 & 9 & 2 & -27 & $3.19 \mathrm{E}-8$ & $1.18 \mathrm{E}-5$ & $>1.00 \mathrm{E}-4$ \\
\hline Non-Small Cell Lung & Cancer & & & & & & & & & & & & & & \\
\hline A549/ATCC & 0.420 & 2.376 & 2.314 & 1.406 & 0.989 & 0.747 & 0.520 & 97 & 50 & 29 & 17 & 5 & 1.05E-7 & $>1.00 \mathrm{E}-4$ & $>1.00 \mathrm{E}-4$ \\
\hline EKVX & 0.568 & 1.628 & 1.538 & 1.193 & 1.013 & 0.910 & 0.553 & 91 & 59 & 42 & 32 & -3 & 3.36E-7 & $8.36 \mathrm{E}-5$ & $>1.00 \mathrm{E}-4$ \\
\hline HOP-62 & 0.895 & 2.673 & 2.512 & 1.784 & 1.730 & 1.534 & 1.084 & 91 & 50 & 47 & 36 & 11 & $1.01 \mathrm{E}-7$ & $>1.00 \mathrm{E}-4$ & $>1.00 \mathrm{E}-4$ \\
\hline HOP-92 & 1.491 & 1.882 & 1.687 & 1.536 & 1.573 & 1.265 & 1.086 & 50 & 12 & 21 & -15 & -27 & $<1.00 \mathrm{E}-8$ & 3.79E-6 & $>1.00 \mathrm{E}-4$ \\
\hline $\mathrm{NCl}-\mathrm{H} 226$ & 1.047 & 2.345 & 2.230 & 1.482 & 1.384 & 1.228 & 0.937 & 91 & 33 & 26 & 14 & -11 & 5.17E-8 & $3.70 \mathrm{E}-5$ & $>1.00 \mathrm{E}-4$ \\
\hline $\mathrm{NCl}-\mathrm{H} 23$ & 0.661 & 1.806 & 1.696 & 1.325 & 0.985 & 0.829 & 0.564 & 90 & 58 & 28 & 15 & -15 & 1.86E-7 & 3.15E-5 & $>1.00 \mathrm{E}-4$ \\
\hline $\mathrm{NCl}-\mathrm{H} 322 \mathrm{M}$ & 0.767 & 2.165 & 2.172 & 1.721 & 1.553 & 1.426 & 1.1 & 100 & 68 & 56 & 47 & 27 & 4.83E-6 & $>1.00 \mathrm{E}-4$ & $>1.00 \mathrm{E}-4$ \\
\hline $\mathrm{NCl}-\mathrm{H} 460$ & 0.296 & 2.704 & 2.780 & 0.796 & 0.480 & 0.344 & 0.250 & 103 & 21 & 8 & 2 & -16 & $4.41 \mathrm{E}-8$ & 1.30E-5 & $>1.00 \mathrm{E}-4$ \\
\hline Colon Cancer & & & & & & & & & & & & & & & \\
\hline & 0.637 & 2.671 & 2.613 & 1.435 & 0.750 & 0.368 & 0.263 & 97 & 39 & 6 & -42 & -59 & $6.52 \mathrm{E}-8$ & $1.31 \mathrm{E}-6$ & $2.96 \mathrm{E}-5$ \\
\hline HCC-2 & 0.633 & 1.990 & 1.961 & 1.504 & 0.853 & 0.794 & 0.530 & 98 & 64 & 16 & 12 & -16 & $1.98 \mathrm{E}-7$ & & $>1.00 \mathrm{E}-4$ \\
\hline HCT-116 & 0.335 & 2.629 & 2.551 & 0.918 & 0.735 & 0.435 & 0.330 & 97 & 25 & 17 & 4 & -1 & $4.51 \mathrm{E}-8$ & $5.55 \mathrm{E}-5$ & > $1.00 \mathrm{E}-4$ \\
\hline HCT-15 & 0.296 & 1.919 & 1.781 & 0.814 & 0.578 & 0.416 & 0.299 & 91 & 32 & 17 & 7 & & 4.97E-8 & $>1.00 \mathrm{E}-4$ & $>1.00 \mathrm{E}-4$ \\
\hline HT29 & 0.442 & 2.685 & 2.635 & 0.918 & 0.691 & 0.639 & 0.424 & 98 & 21 & 11 & 9 & -4 & $4.21 \mathrm{E}-8$ & $4.75 \mathrm{E}-5$ & $>1.00 \mathrm{E}-4$ \\
\hline KM12 & 0.854 & 3.324 & 3.312 & 1.551 & 1.407 & 1.038 & 0.507 & 100 & 28 & 22 & 7 & -41 & 4.95E-8 & $1.43 \mathrm{E}-5$ & $>1.00 \mathrm{E}-4$ \\
\hline SW-620 & 0.321 & 1.953 & 1.851 & 0.792 & 0.727 & 0.615 & 0.378 & 94 & 29 & 25 & 18 & 3 & 4.72E-8 & $>1.00 E-4$ & $>1.00 \mathrm{E}-4$ \\
\hline CNS Cancer & & & & & & & & & & & & & & & \\
\hline SF-26 & 0.964 & $\begin{array}{l}2.622 \\
3173\end{array}$ & 2.565 & 1.985 & 1.948 & 1.396 & 1.049 & 97 & 62 & 59 & 26 & 5 & 1.91E-6 & $>1.00 \mathrm{E}-4$ & $>1.00 \mathrm{E}-4$ \\
\hline SF-29 & 0.980 & 3.173 & 3.047 & 1.574 & 1.339 & 1.299 & 0.507 & 94 & 27 & 16 & 15 & -48 & & & $>1.00 \mathrm{E}-4$ \\
\hline SF-53S & 1.014 & 2.805 & 2.739 & 1.143 & 0.848 & 0.866 & 0.694 & 96 & 7 & -16 & -15 & -32 & & & $>1.00 \mathrm{E}-4$ \\
\hline SNB-1 & 0.546 & 1.889 & 1.786 & 0.995 & 0.841 & 0.828 & 0.717 & 92 & 33 & 22 & 21 & 13 & $5.23 \mathrm{E}$ & & $>1.00 \mathrm{E}-4$ \\
\hline SNB-75 & 1.164 & 2.158 & 2.040 & 1.249 & 1.269 & 1.375 & 1.142 & 88 & 9 & 11 & 21 & -2 & & & $>1.00 \mathrm{E}-4$ \\
\hline U251 & 0.429 & 2.041 & 1.980 & 1.154 & 0.838 & 0.805 & 0.453 & 96 & 45 & 25 & 23 & 1 & 7.96E-8 & $>1.00 E-4$ & $>1.00 \mathrm{E}-4$ \\
\hline Melanoma & & & & & & & & & & & & & & & \\
\hline & 0.305 & 1.971 & 1.833 & 0.892 & 0.818 & 0.548 & 0.242 & 92 & 35 & 31 & 15 & -21 & $5.48 \mathrm{E}-8$ & $2.58 \mathrm{E}-5$ & $>1.00 \mathrm{E}-4$ \\
\hline MALME-3M & 0.832 & 2.235 & 2.214 & 1.579 & 1.700 & 1.613 & 0.73 & 99 & 53 & 62 & 56 & -12 & $1.21 \mathrm{E}-5$ & & $>1.00 \mathrm{E}-4$ \\
\hline M14 & 0.545 & 1.940 & 1.881 & 1.096 & 0.679 & 0.761 & 0.403 & 96 & 39 & 10 & 15 & -26 & & & $>1.00 \mathrm{E}-4$ \\
\hline MDA-MB-435 & 0.575 & 2.534 & 2.248 & 0.539 & 0.376 & 0.560 & 0.41 & 85 & -6 & -35 & -3 & -28 & & & $>1.00 \mathrm{E}-4$ \\
\hline SK-MEL-2 & 1.298 & 2.560 & 2.466 & 1.978 & 2.100 & 1.948 & 1.0 & 93 & 54 & 64 & 51 & -18 & 1.05 & & $>1.00 \mathrm{E}-4$ \\
\hline SK-MEL- & 0.553 & 1.723 & 1.673 & 1.169 & 1.168 & 1.094 & 0.55 & 96 & 53 & 53 & 46 & & 2.51 & $>1.00 \mathrm{E}-4$ & $>1.00 \mathrm{E}-4$ \\
\hline SK-ME & 1.089 & 3.215 & 3.119 & 1.934 & 1.608 & 0.9 & 0.0 & 95 & 40 & 24 & -14 & -94 & & & $2.84 \mathrm{E}-5$ \\
\hline UACC-257 & 1.013 & 2.520 & 2.300 & 1.831 & 2.061 & 1.703 & 0.8 & 85 & 54 & 70 & 46 & -18 & & & $>1.00 \mathrm{E}-4$ \\
\hline UACC-62 & 0.918 & 2.600 & 2.384 & 1.259 & 1.259 & 1.181 & 0.491 & 87 & 20 & 20 & 16 & -47 & 3.60E-8 & $1.78 \mathrm{E}-5$ & $>1.00 \mathrm{E}-4$ \\
\hline Ovari & & & & & & & & & & & & & & & \\
\hline & 0.342 & 1.864 & 1.842 & 0.988 & 0.759 & 0.551 & 0.382 & 99 & 42 & 27 & 14 & 3 & & $1.00 \mathrm{E}-4$ & $>1.00 \mathrm{E}-4$ \\
\hline & 0.626 & 1.929 & 2.041 & 0.826 & 0.729 & 0.698 & 0.48 & 109 & 15 & 8 & 6 & -23 & & & $>1.00 \mathrm{E}-4$ \\
\hline & 0.761 & 2.090 & 2.116 & 1.363 & 1.344 & 1.092 & 0.8 & 102 & 45 & 44 & 25 & 8 & & & $>1.0$ \\
\hline & 0.491 & 1.376 & 1.374 & 1.16 & 0.822 & 0.782 & 0.6 & 100 & 76 & 37 & 33 & 16 & & & $>1.0$ \\
\hline & 0.738 & 2.938 & 2.881 & 2.232 & 1.607 & 1.337 & 0.87 & 97 & 68 & 40 & 27 & & & & $>1.00 \mathrm{E}-4$ \\
\hline NCI/ADR-F & 0.514 & 1.768 & 1.679 & 0.753 & 0.580 & 0.513 & 0.444 & 93 & 19 & 5 & & -14 & & & $>1.00 \mathrm{E}-4$ \\
\hline SK-OV-3 & 1.344 & 2.546 & 2.617 & 2.034 & 2.018 & 1.875 & 1.380 & 106 & 57 & 56 & 44 & 3 & $3.21 \mathrm{E}-6$ & $>1.00 E-4$ & $>1.00 \mathrm{E}-4$ \\
\hline Renal Cancer & & & & & & & & & & & & & & & \\
\hline & 0.678 & 2.591 & 2.504 & 1.391 & 1.214 & 1.004 & 0.65 & 95 & 37 & 28 & 17 & -3 & $6.04 \mathrm{E}-8$ & $6.87 \mathrm{E}-5$ & $>1.00 \mathrm{E}-4$ \\
\hline & & & & 1.6 & 1.6 & & & 78 & -8 & -5 & -10 & -17 & & & \\
\hline & & & & 1.0 & 1.0 & & & $9 \varepsilon$ & 36 & 39 & 19 & 2 & & & \\
\hline & & & 2.5 & 1.6 & 1.7 & & & 76 & 36 & 39 & 15 & -3 & & $6.67 \mathrm{E}-5$ & \\
\hline & & & & 1.0 & 0.8 & & 0.8 & 83 & -2 & -21 & 3 & -20 & & & \\
\hline & & & & & 2.2 & & 1.1 & 95 & 70 & 47 & 21 & -13 & & & \\
\hline & 1.107 & 2.012 & 1.924 & 1.875 & 1.951 & & 1.135 & 90 & 85 & 93 & 72 & 3 & & $>1.00 \mathrm{E}-4$ & \\
\hline บO-31 & 0.716 & 2.321 & 2.099 & 1.500 & 1.495 & 1.168 & 0.786 & 86 & 49 & 49 & 28 & 4 & $9.31 \mathrm{E}-8$ & $>1.00 \mathrm{E}-4$ & $>1.00 \mathrm{E}-4$ \\
\hline Prostate Cancer & & & & & & & & & & & & & & & \\
\hline & 0.835 & $\begin{array}{l}2.129 \\
1744\end{array}$ & $\begin{array}{l}2.003 \\
1785\end{array}$ & $\begin{array}{l}1.214 \\
1111\end{array}$ & 1.015 & 1.031 & 0.734 & 90 & $\begin{array}{l}29 \\
53\end{array}$ & $\begin{array}{l}14 \\
18\end{array}$ & 15 & -12 & $\begin{array}{l}4.57 \mathrm{E}-8 \\
124 \mathrm{~F}-7\end{array}$ & 3.59E-5 & $>1.00 \mathrm{E}-4$ \\
\hline Breas & 0.453 & 1.744 & 1.185 & 1.141 & 0.686 & 0.570 & 0.493 & 103 & 53 & 18 & & 3 & $1.24 \mathrm{E}-7$ & 1.00E-4 & $>1.00 \mathrm{E}-4$ \\
\hline $\begin{array}{l}\text { ast Cancer } \\
\text { F7 }\end{array}$ & & & & & & & & & 17 & 17 & & & & & \\
\hline A-MB-231/ATCC & $=0.685$ & & & $\begin{array}{l}0 . \\
1.2\end{array}$ & 0.9 & 0.7 & 0.5 & 101 & 72 & 31 & 14 & -23 & & & \\
\hline HS 57 & & 1.8 & 1.7 & 1.1 & 1.0 & & 0.8 & 96 & 34 & 21 & 24 & 1 & $5.47 \mathrm{E}-8$ & & $>1.00 \mathrm{E}-4$ \\
\hline BT-5 & 1.2 & 2.2 & 2.2 & 1.7 & 1.7 & 1.4 & 0.8 & 96 & 48 & 51 & 23 & -34 & & & $>1.00 \mathrm{E}-4$ \\
\hline T-47D & 1.008 & 2.553 & 2.404 & 1.536 & 1.830 & 1.584 & 1.055 & 90 & 34 & 53 & 37 & 3 & & $1.00 \mathrm{E}-4$ & $>1.00 \mathrm{E}-4$ \\
\hline MDA-MB-468 & 0.703 & 1.950 & 1.863 & 0.886 & 0.831 & 0.653 & 0.442 & 93 & 15 & 10 & -7 & -37 & $3.54 \mathrm{E}-8$ & 3.87E-6 & $>1.00 \mathrm{E}-4$ \\
\hline
\end{tabular}


In-Vitro Testing Results

\begin{tabular}{|l|l|l|l|}
\hline NSC : D - 826501/1 & Experiment ID : 2101NS68 & Test Type : 08 & Units : Molar \\
\hline Report Date : March 24, 2021 & Test Date : January 04, 2021 & QNS : & MC : \\
\hline COMI : KLB2-51 & Stain Reagent : SRB Dual-Pass Related & SSPL : 1C1X & \\
\hline
\end{tabular}

Log10 Concentration

\begin{tabular}{|c|c|c|c|c|c|c|c|c|c|c|c|c|c|c|c|}
\hline \multirow[b]{2}{*}{ Panel/Cell Line } & \multirow{2}{*}{$\begin{array}{l}\text { Time } \\
\text { Zero }\end{array}$} & \multirow[b]{2}{*}{ Ctrl } & \multicolumn{5}{|c|}{ Mean Optical Densities } & \multicolumn{5}{|c|}{ Percent Growth } & \multirow[b]{2}{*}{ GI50 } & \multirow[b]{2}{*}{ TGI } & \\
\hline & & & -8.0 & -7.0 & -6.0 & -5.0 & -4.0 & -8.0 & -7.0 & -6.0 & -5.0 & -4.0 & & & LC50 \\
\hline Leukemia & & & & & & & & & & & & & & & \\
\hline CCRF-CEM & 0.488 & 1.691 & 1.667 & 0.875 & 0.608 & 0.593 & 0.494 & 98 & 32 & 10 & 9 & & $5.36 \mathrm{E}-8$ & $>1.00 \mathrm{E}-4$ & $>1.00 \mathrm{E}-4$ \\
\hline HL-60(TB) & 0.693 & 2.287 & 2.170 & 0.550 & 0.496 & 0.392 & 0.314 & 93 & -21 & -28 & -44 & -55 & $2.38 \mathrm{E}-8$ & 6.57E-8 & $3.81 \mathrm{E}-5$ \\
\hline $\mathrm{K}-562$ & 0.282 & 2.136 & 2.169 & 0.564 & 0.430 & 0.350 & 0.256 & 102 & 15 & 8 & 4 & -9 & 3.96E-8 & $1.92 \mathrm{E}-5$ & $>1.00 \mathrm{E}-4$ \\
\hline MOLT-4 & 0.721 & 2.439 & 2.354 & 1.519 & 1.036 & 0.812 & 0.503 & 95 & 46 & 18 & 5 & -30 & $8.45 \mathrm{E}-8$ & $1.41 \mathrm{E}-5$ & $>1.00 \mathrm{E}-4$ \\
\hline RPMI-8226 & 1.118 & 2.302 & 2.399 & 1.395 & 1.192 & 0.982 & 0.729 & 108 & 23 & 6 & -12 & -35 & $4.86 \mathrm{E}-8$ & $2.18 \mathrm{E}-6$ & $>1.00 \mathrm{E}-4$ \\
\hline & 0.683 & 2.795 & 2.239 & 0.885 & 0.823 & 0.586 & 0.443 & 74 & 10 & 7 & -14 & -35 & $2.34 \mathrm{E}-8$ & $2.08 \mathrm{E}-6$ & $>1.00 \mathrm{E}-4$ \\
\hline Non-Small Cell Lung & Cancer & & & & & & & & & & & & & & \\
\hline A549/ATCC & 0.420 & 2.472 & 2.367 & 1.503 & 0.961 & 0.755 & 0.534 & 95 & 53 & 26 & 16 & 6 & $1.27 \mathrm{E}-7$ & $>1.00 \mathrm{E}-4$ & $>1.00 \mathrm{E}-4$ \\
\hline EKVX & 0.568 & 1.609 & 1.569 & 1.174 & 1.015 & 0.919 & 0.616 & 96 & 58 & 43 & 34 & 5 & $3.43 \mathrm{E}-7$ & $>1.00 \mathrm{E}-4$ & $>1.00 \mathrm{E}-4$ \\
\hline HOP-62 & 0.895 & 2.722 & 2.521 & 1.912 & 1.645 & 1.592 & 1.094 & 89 & 56 & 41 & 38 & 11 & 2.44E-7 & $>1.00 \mathrm{E}-4$ & $>1.00 \mathrm{E}-4$ \\
\hline HOP-92 & 1.491 & 2.099 & 1.973 & 1.838 & 1.846 & 1.525 & 1.156 & 79 & 57 & 58 & 6 & -22 & $1.44 \mathrm{E}-6$ & $1.58 \mathrm{E}-5$ & $>1.00 \mathrm{E}-4$ \\
\hline $\mathrm{NCl}-\mathrm{H} 226$ & 1.047 & 2.369 & 2.222 & 1.752 & 1.485 & 1.228 & 1.009 & 89 & 53 & 33 & 14 & -4 & $1.46 \mathrm{E}-7$ & $6.14 \mathrm{E}-5$ & $>1.00 \mathrm{E}-4$ \\
\hline $\mathrm{NCl}-\mathrm{H} 23$ & 0.661 & 1.883 & 1.742 & 1.447 & 1.132 & 0.925 & 0.672 & 88 & 64 & 38 & 22 & 1 & $3.58 \mathrm{E}-7$ & $>1.00 \mathrm{E}-4$ & $>1.00 \mathrm{E}-4$ \\
\hline $\mathrm{NCl}-\mathrm{H} 322 \mathrm{M}$ & 0.767 & 2.189 & 2.104 & 1.892 & 1.450 & 1.516 & 1.344 & 94 & 79 & 48 & 53 & 41 & & $>1.00 \mathrm{E}-4$ & $>1.00 \mathrm{E}-4$ \\
\hline $\mathrm{NCl}-\mathrm{H} 460$ & 0.296 & 2.777 & 2.858 & 0.906 & 0.557 & 0.337 & 0.266 & 103 & 25 & 11 & 2 & -10 & $4.75 \mathrm{E}-8$ & $1.37 \mathrm{E}-5$ & $>1.00 \mathrm{E}-4$ \\
\hline Colon Cancer & & & & & & & & & & & & & & & \\
\hline $\begin{array}{l}\text { COLO } \\
\text { HCC-? }\end{array}$ & 0.637 & $\begin{array}{l}2.711 \\
2030\end{array}$ & $\begin{array}{l}2.698 \\
1.959\end{array}$ & 1.570 & 0.816 & 0.564 & 0.423 & 99 & 45 & 9 & -11 & -34 & 8.09E-8 & 2.68E-6 & $>1.00 \mathrm{E}-4$ \\
\hline HCC-2998 & 0.633 & 2.030 & $\begin{array}{l}1.959 \\
2.48\end{array}$ & 1.610 & 1.149 & 0.848 & 0.637 & 95 & 70 & 37 & 15 & & 4.01E-7 & $>1.00 \mathrm{E}-4$ & $>1.00 \mathrm{E}-4$ \\
\hline $\begin{array}{l}\text { HCI-116 } \\
\text { HCT } 15\end{array}$ & 0.335 & 2.641 & 2.484 & 0.901 & 0.852 & 0.413 & 0.358 & 93 & 25 & 22 & $\begin{array}{r}3 \\
15\end{array}$ & 1 & 4.26E-8 & $>1.00 \mathrm{E}-4$ & $>1.00 \mathrm{E}-4$ \\
\hline $\begin{array}{l}\mathrm{ACT}-1 \\
\mathrm{HT} 2 \mathrm{9}\end{array}$ & 0.296 & 1.986 & 1.857 & 0.853 & 0.778 & 0.542 & 0.376 & 92 & 33 & 29 & 15 & $\begin{array}{r}5 \\
13\end{array}$ & 5.16E-8 & $>1.00 \mathrm{E}-4$ & $>1.00 \mathrm{E}-4$ \\
\hline KM1 & 0.442 & 2.715 & 2.672 & 0.927 & 0.698 & 0.583 & 0.385 & 98 & 21 & 11 & 6 & -13 & 4.23E-8 & $2.11 \mathrm{E}-5$ & $>1.00 \mathrm{E}-4$ \\
\hline $\begin{array}{l}\text { KM12 } \\
\text { SW-620 }\end{array}$ & 0.854 & 3.316 & $\begin{array}{l}3.282 \\
2017\end{array}$ & $\begin{array}{l}1.745 \\
1059\end{array}$ & 1.601 & 1.226 & $\begin{array}{l}0.767 \\
0.433\end{array}$ & $\begin{array}{r}99 \\
103\end{array}$ & $\begin{array}{l}36 \\
45\end{array}$ & $\begin{array}{l}30 \\
32\end{array}$ & $\begin{array}{l}15 \\
21\end{array}$ & $\begin{array}{r}-10 \\
7\end{array}$ & 6.01E-8 & $\begin{array}{l}3.94 \mathrm{E}-5 \\
>\end{array}$ & $>1.00 \mathrm{E}-4$ \\
\hline & & 1.000 & & 1.000 & 0.040 & 0.070 & 0.400 & & & 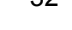 & & $t$ & $0.10 E-0$ & $1.00 E^{-4}$ & $>1.00 \mathrm{E}-4$ \\
\hline CNS Cancer & & & & & & & & & & & & & & & \\
\hline SF-295 & $\begin{array}{l}0.964 \\
0.980\end{array}$ & $\begin{array}{l}2.6 / 3 \\
3.217\end{array}$ & 3.118 & 1.744 & 1.460 & 1.408 & 0.723 & $\begin{array}{l}94 \\
96\end{array}$ & 34 & 21 & 19 & $\begin{array}{r}4 \\
-26\end{array}$ & $\begin{array}{l}1.55 \mathrm{E}-6 \\
5.52 \mathrm{E}-8\end{array}$ & $\begin{array}{r}>1.00 \mathrm{E}-4 \\
2.64 \mathrm{E}-5\end{array}$ & $\begin{array}{l}>1.000-4 \\
>1.00 E-4\end{array}$ \\
\hline SF-539 & 1.014 & 2.826 & $\begin{array}{l}2.726 \\
2.726\end{array}$ & 1.592 & 0.712 & 0.670 & 0.587 & 94 & $\begin{array}{l}34 \\
32\end{array}$ & -30 & -34 & -42 & $5.14 \mathrm{E}-8$ & $3.29 \mathrm{E}-7$ & $>1.00 \mathrm{E}-4$ \\
\hline SNB-19 & 0.546 & 1.847 & 1.734 & 1.053 & 0.852 & 0.780 & 0.749 & 91 & 39 & 24 & 18 & 16 & $6.15 \mathrm{E}-8$ & $>1.00 \mathrm{E}-4$ & $>1.00 \mathrm{E}-4$ \\
\hline SNB-75 & 1.164 & 2.147 & 1.974 & 1.505 & 1.017 & 1.237 & 1.052 & 82 & 35 & -13 & 7 & -10 & $4.78 \mathrm{E}-8$ & & $>1.00 \mathrm{E}-4$ \\
\hline U251 & 0.429 & 2.081 & 1.997 & 1.144 & 0.902 & 0.810 & 0.444 & 95 & 43 & 29 & 23 & 1 & $7.41 \mathrm{E}-8$ & $>1.00 \mathrm{E}-4$ & $>1.00 \mathrm{E}-4$ \\
\hline Melanoma & & & & & & & & & & & & & & & \\
\hline LOX IMVI & 0.305 & 1.967 & 1.905 & 0.973 & 0.865 & 0.423 & 0.199 & 96 & 40 & 34 & 7 & -35 & $6.68 \mathrm{E}-8$ & 1.47E-5 & $>1.00 \mathrm{E}-4$ \\
\hline MALM & 0.832 & 2.274 & 2.270 & 1.613 & 1.736 & 1.630 & 0.614 & 100 & 54 & 63 & 55 & -26 & 1.16E-5 & 4.76E-5 & $>1.00 \mathrm{E}-4$ \\
\hline M14 & 0.545 & 1.919 & 1.825 & 1.021 & 0.652 & 0.738 & 0.392 & 93 & 35 & 8 & 14 & -28 & $5.46 \mathrm{E}-8$ & 2.15E-5 & $>1.00 \mathrm{E}-4$ \\
\hline MDA-MB-435 & 0.575 & 2.556 & 2.221 & 0.535 & 0.277 & 0.417 & 0.369 & 83 & -7 & -52 & -27 & -36 & $2.33 \mathrm{E}-8$ & $8.35 \mathrm{E}-8$ & \\
\hline SK-MEL-2 & 1.298 & 2.535 & 2.468 & 1.936 & 1.934 & 1.890 & 0.891 & 95 & 52 & 51 & 48 & -31 & $2.49 \mathrm{E}-6$ & $4.02 \mathrm{E}-5$ & $>1.00 \mathrm{E}-4$ \\
\hline SK-MEL & 0.553 & 1.640 & 1.576 & 1.134 & 1.098 & 1.090 & 0.617 & 94 & 53 & 50 & 49 & 6 & $1.60 \mathrm{E}-6$ & $>1.00 \mathrm{E}-4$ & $>1.00 \mathrm{E}-4$ \\
\hline SK-MEL-5 & 1.089 & 3.253 & 3.204 & 2.102 & 1.752 & 1.085 & 0.071 & 98 & 47 & 31 & & -94 & $8.66 \mathrm{E}-8$ & $9.70 \mathrm{E}-6$ & $3.41 \mathrm{E}-5$ \\
\hline UACC-257 & 1.013 & 2.428 & 2.278 & 1.904 & 2.032 & 1.798 & 0.855 & 89 & 63 & 72 & 55 & -16 & $1.19 \mathrm{E}-5$ & $6.03 \mathrm{E}-5$ & $>1.00 \mathrm{E}-4$ \\
\hline UACC- 62 & 0.918 & 2.637 & 2.450 & 1.369 & 1.363 & 1.196 & 0.615 & 89 & 26 & 26 & 16 & -33 & $4.19 \mathrm{E}-8$ & $2.13 \mathrm{E}-5$ & $>1.00 \mathrm{E}-4$ \\
\hline Ovarian Cancer & & & & & & & & & & & & & & & \\
\hline & 0.342 & 1.862 & 1.760 & 0.994 & 0.771 & 0.602 & 0.472 & 93 & 43 & 28 & 17 & 9 & 7.22E-8 & $>1.00 \mathrm{E}-4$ & $>1.00 \mathrm{E}-4$ \\
\hline & 0.626 & 1.920 & 1.988 & 0.864 & 0.676 & 0.714 & 0.433 & 105 & 18 & 4 & 7 & -31 & & $1.52 \mathrm{E}-5$ & $>1.00 \mathrm{E}-4$ \\
\hline & 0.761 & 2.082 & 2.058 & 1.615 & 1.353 & 1.039 & 0.8 & 98 & 65 & 45 & 21 & 7 & & $>1.0$ & $>1.00 \mathrm{E}-4$ \\
\hline & $0.4 \varsigma$ & 1.37 & 1.313 & 1.208 & 0.784 & 0.7 & 0.6 & 93 & 81 & 33 & 24 & 22 & & & $>1.00 \mathrm{E}-4$ \\
\hline & 0.73 & 2.88 & 2.895 & 2.341 & 1.699 & 1.33 & 0.8 & 100 & 75 & 45 & 28 & 5 & & & $>1.00 \mathrm{E}-4$ \\
\hline NCI/ADR-RES & 0.514 & 1.802 & 1.720 & 0.909 & 0.718 & 0.635 & 0.4 & 94 & 31 & 16 & 9 & -12 & & & $>1.00 \mathrm{E}-4$ \\
\hline SK-OV-3 & 1.344 & 2.606 & 2.585 & 2.172 & 1.978 & 1.974 & 1.484 & 98 & 66 & 50 & 50 & 11 & $6.49 \mathrm{E}-6$ & $>1.00 \mathrm{E}-4$ & $>1.00 \mathrm{E}-4$ \\
\hline Renal Cancer & & & & & & & & & & & & & & & \\
\hline & 0.678 & 2.509 & 2.401 & 1.641 & 1.319 & 0.809 & 0.525 & 94 & 53 & 35 & 7 & -23 & $1.40 \mathrm{E}-7$ & $1.74 \mathrm{E}-5$ & $>1.00 \mathrm{E}-4$ \\
\hline & 1.770 & 2.480 & 2.323 & 1.752 & 1.625 & 1.46 & 1.22 & 78 & -1 & -8 & -17 & -31 & & & $>1.00 \mathrm{E}-4$ \\
\hline $\mathrm{ACHN}$ & 0.474 & 1.974 & 1.904 & 1.22 & 1.126 & 0.783 & 0.43 & 95 & 50 & 43 & 21 & -8 & & & $>1.00 \mathrm{E}-4$ \\
\hline & 0.918 & 3.0 & 2.68 & 1.7 & 1.696 & 1.3 & 0.95 & 84 & 41 & 37 & 21 & & & $>1.0$ & $>1.00 \mathrm{E}-4$ \\
\hline & 1.0 & 1.52 & 1.45 & 1.1 & 0.769 & 0.8 & 0.73 & 84 & 22 & -28 & -20 & -32 & & & $>1.00 \mathrm{E}-4$ \\
\hline SN12C & 1.323 & 3.3 & 3.202 & 2.8 & 2.48 & 1.6 & 1.16 & 94 & 77 & 58 & 18 & -12 & $=-6$ & $3.86 \mathrm{E}-5$ & $>1.00 \mathrm{E}-4$ \\
\hline TK-1 & 1.107 & 2.020 & 1.91 & 1.8 & 1.88 & 1.758 & 1.21 & 88 & 87 & 85 & 71 & 11 & & $1.00 \mathrm{E}-4$ & $1.00 \mathrm{E}-4$ \\
\hline UO-31 & 0.716 & 2.319 & 2.105 & 1.716 & 1.538 & 1.215 & 0.791 & 87 & 62 & 51 & 31 & 5 & 1.16E-6 & $>1.00 \mathrm{E}-4$ & $>1.00 \mathrm{E}-4$ \\
\hline Prostate Cancer & & & & & & & & & & & & & & & \\
\hline & 0.835 & 2.426 & 2.357 & 1.738 & 1.281 & 1.150 & 0.751 & 96 & 57 & 28 & 20 & -10 & $1.71 \mathrm{E}-7$ & & $>1.00 \mathrm{E}-4$ \\
\hline DU-145 & 0.453 & 1.757 & 1.814 & 1.396 & 0.678 & 0.501 & 0.448 & 104 & 72 & 17 & 4 & -1 & $2.54 \mathrm{E}-7$ & $5.88 \mathrm{E}-5$ & $>1.00 \mathrm{E}-4$ \\
\hline Breast Ca & & & & & & & & & & & & & & & \\
\hline MCF7 & 0.480 & 2.349 & 2.164 & 0.909 & 0.834 & 0.668 & 0.477 & 90 & 23 & 19 & 10 & & & & \\
\hline MDA-N & 0.685 & 1.540 & 1.573 & 1.234 & 0.995 & 0.725 & 0.542 & 104 & 64 & 36 & 5 & -21 & & $1.52 \mathrm{E}-5$ & \\
\hline HS 57 & 0.817 & 1.799 & 1.722 & 1.321 & 1.082 & 1.009 & 0.84 & 92 & 51 & 27 & 20 & 3 & 1.14 & $1.00 \mathrm{E}$ & $1.00 \mathrm{E}-4$ \\
\hline BT-54 & 1.257 & 2.259 & 2.127 & 1.753 & 1.730 & $1.3 \mathrm{~s}$ & 0.87 & 87 & 50 & 47 & 13 & -30 & & & 100 \\
\hline T-47[ & 1.008 & 2.504 & 2.417 & 1.636 & 1.635 & 1.471 & 0.983 & 94 & 42 & 42 & 31 & -3 & 7.02E-8 & $8.40 \mathrm{E}-5$ & $.00 \mathrm{E}-4$ \\
\hline MDA-MB-468 & 0.703 & 1.928 & 1.782 & 0.937 & 0.801 & 0.567 & 0.466 & 88 & 19 & 8 & -19 & -34 & 3.56E-8 & $1.95 \mathrm{E}-6$ & $>1.00 \mathrm{E}-4$ \\
\hline
\end{tabular}


In-Vitro Testing Results

\begin{tabular}{|l|l|l|l|}
\hline NSC : D - 826495/1 & Experiment ID : 2101NS68 & Test Type : 08 & Units : Molar \\
\hline Report Date : March 24, 2021 & Test Date : January 04, 2021 & QNS : & MC : \\
\hline COMI : KLB2-45 & Stain Reagent : SRB Dual-Pass Related & SSPL : 1C1X & \\
\hline
\end{tabular}

Log10 Concentration

\begin{tabular}{|c|c|c|c|c|c|c|c|c|c|c|c|c|c|c|c|}
\hline \multirow[b]{2}{*}{ Panel/Cell Line } & \multirow{2}{*}{$\begin{array}{l}\text { Time } \\
\text { Zero }\end{array}$} & \multirow[b]{2}{*}{ Ctrl } & \multicolumn{5}{|c|}{ Mean Optical Densities } & \multicolumn{5}{|c|}{ Percent Growth } & \multirow[b]{2}{*}{ G150 } & \multirow[b]{2}{*}{ TGI } & \\
\hline & & & -8.0 & -7.0 & -6.0 & -5.0 & -4.0 & -8.0 & -7.0 & -6.0 & -5.0 & -4.0 & & & LC50 \\
\hline Leukemia & & & & & & & & & & & & & & & \\
\hline CCRF-CEM & 0.488 & 1.699 & 1.470 & 1.216 & 0.698 & 0.665 & 0.645 & 81 & 60 & 17 & 15 & 13 & $1.72 \mathrm{E}-7$ & $>1.00 \mathrm{E}-4$ & $>1.00 \mathrm{E}-4$ \\
\hline HL-60(TB) & 0.693 & 2.466 & 2.414 & 0.788 & 0.489 & 0.367 & 0.473 & 97 & 5 & -30 & -47 & -32 & 3.26E-8 & 1.42E-7 & $>1.00 \mathrm{E}-4$ \\
\hline $\mathrm{K}-562$ & 0.282 & 2.497 & 2.433 & 0.691 & 0.435 & 0.379 & 0.358 & 97 & 18 & 7 & 4 & 3 & 3.97E-8 & $>1.00 \mathrm{E}-4$ & $>1.00 \mathrm{E}-4$ \\
\hline MOLT-4 & 0.721 & 2.415 & 2.491 & 1.938 & 0.965 & 0.832 & 0.897 & 104 & 72 & 14 & 7 & 10 & $2.40 \mathrm{E}-7$ & $>1.00 \mathrm{E}-4$ & $>1.00 \mathrm{E}-4$ \\
\hline RPMI-8226 & 1.118 & 2.422 & 2.520 & 2.009 & 1.263 & 0.927 & 1.101 & 108 & 68 & 11 & -17 & -2 & 2.09E-7 & 2.47E-6 & $>1.00 \mathrm{E}-4$ \\
\hline & 0.683 & 2.806 & 2.487 & 1.004 & 0.873 & 0.630 & 0.775 & 85 & 15 & 9 & -8 & 4 & 3.17E-8 & & $>1.00 \mathrm{E}-4$ \\
\hline Non-Small Cell Lung & Cancer & & & & & & & & & & & & & & \\
\hline A549/ATCC & 0.420 & 2.456 & 2.393 & 1.829 & 1.016 & 0.770 & 0.771 & 97 & 69 & 29 & 17 & 17 & $3.02 \mathrm{E}-7$ & $>1.00 \mathrm{E}-4$ & $>1.00 \mathrm{E}-4$ \\
\hline EKVX & 0.568 & 1.604 & 1.551 & 1.304 & 0.978 & 0.843 & 0.873 & 95 & 71 & 40 & 27 & 29 & 4.65E-7 & $>1.00 \mathrm{E}-4$ & $>1.00 \mathrm{E}-4$ \\
\hline HOP-62 & 0.895 & 2.758 & 2.578 & 2.142 & 1.764 & 1.614 & 1.152 & 90 & 67 & 47 & 39 & 14 & $6.81 \mathrm{E}-7$ & $>1.00 \mathrm{E}-4$ & $>1.00 \mathrm{E}-4$ \\
\hline $\mathrm{NCl}-\mathrm{H} 226$ & 1.047 & 2.291 & 2.187 & 1.873 & 1.342 & 0.963 & 1.139 & 92 & 66 & 24 & -8 & 7 & $2.42 \mathrm{E}-7$ & & $>1.00 \mathrm{E}-4$ \\
\hline $\mathrm{NCl}-\mathrm{H} 23$ & 0.661 & 1.870 & 1.692 & 1.596 & 1.040 & 0.815 & 0.872 & 85 & 77 & 31 & 13 & 17 & 3.93E-7 & $>1.00 \mathrm{E}-4$ & $>1.00 \mathrm{E}-4$ \\
\hline $\mathrm{NCl}-\mathrm{H} 322 \mathrm{M}$ & 0.767 & 2.269 & 2.247 & 2.185 & 1.555 & 1.548 & 1.371 & 99 & 94 & 52 & 52 & 40 & $1.48 \mathrm{E}-5$ & $>1.00 \mathrm{E}-4$ & $>1.00 \mathrm{E}-4$ \\
\hline $\mathrm{NCl}-\mathrm{H} 460$ & 0.296 & 2.700 & 2.825 & 2.001 & 0.486 & 0.253 & 0.389 & 105 & 71 & 8 & -15 & 4 & $2.15 \mathrm{E}-7$ & & $>1.00 \mathrm{E}-4$ \\
\hline Colon Cancer & & & & & & & & & & & & & & & \\
\hline & 0.637 & 2.757 & 2.728 & 2.400 & 0.891 & 0.609 & 0.790 & 99 & 83 & 12 & -4 & 7 & 2.92E-7 & & $>1.00 \mathrm{E}-4$ \\
\hline HCC-2998 & 0.633 & 2.023 & 2.014 & 1.628 & 0.963 & 0.743 & 0.806 & 99 & 72 & 24 & 8 & 12 & $2.82 \mathrm{E}-7$ & $>1.00 \mathrm{E}-4$ & $>1.00 \mathrm{E}-4$ \\
\hline HCT-116 & 0.335 & 2.661 & 2.559 & 1.549 & 0.805 & 0.455 & 0.596 & 96 & 52 & 20 & 5 & 11 & 1.17E-7 & $>1.00 \mathrm{E}-4$ & $>1.00 \mathrm{E}-4$ \\
\hline HCT-15 & 0.296 & 2.037 & 1.954 & 1.007 & 0.717 & 0.494 & 0.561 & 95 & 41 & 24 & 11 & 15 & $6.78 \mathrm{E}-8$ & $>1.00 \mathrm{E}-4$ & $>1.00 \mathrm{E}-4$ \\
\hline HT29 & 0.442 & 2.703 & 2.646 & 1.753 & 0.723 & 0.575 & 0.650 & 97 & 58 & 12 & 6 & 9 & $1.50 \mathrm{E}-7$ & $>1.00 \mathrm{E}-4$ & $>1.00 \mathrm{E}-4$ \\
\hline KM12 & 0.854 & 3.343 & 3.310 & 2.316 & 1.623 & 1.123 & 1.310 & 99 & 59 & 31 & 11 & 18 & $2.06 \mathrm{E}-7$ & $>1.00 \mathrm{E}-4$ & $>1.00 \mathrm{E}-4$ \\
\hline SW-620 & 0.321 & 1.976 & 1.931 & 1.025 & 0.762 & 0.652 & 0.604 & 97 & 43 & 27 & 20 & 17 & $7.30 \mathrm{E}-8$ & $>1.00 \mathrm{E}-4$ & $>1.00 \mathrm{E}-4$ \\
\hline CNS Cancer & & & & & & & & & & & & & & & \\
\hline SF-26 & 0.964 & 2.643 & 2.607 & 2.298 & 1.943 & 1.287 & 1.421 & 98 & 79 & 58 & 19 & 27 & 1.63E-6 & $>1.00 \mathrm{E}-4$ & $>1.00 \mathrm{E}-4$ \\
\hline SF-295 & 0.980 & 3.246 & 3.130 & 2.038 & 1.419 & 1.432 & 0.982 & 95 & 47 & 19 & 20 & & $8.53 \mathrm{E}-8$ & $>1.00 \mathrm{E}-4$ & $>1.00 \mathrm{E}-4$ \\
\hline SF-539 & 1.014 & 2.844 & 2.818 & 2.124 & 0.902 & 0.889 & 0.759 & 99 & 61 & -11 & -12 & -25 & $1.41 \mathrm{E}-7$ & 7.00E-7 & $>1.00 \mathrm{E}-4$ \\
\hline SNB-19 & 0.546 & 1.895 & 1.832 & 1.400 & 0.887 & 0.868 & 0.687 & 95 & 63 & 25 & 24 & 10 & 2.24E-7 & $>1.00 \mathrm{E}-4$ & $>1.00 \mathrm{E}-4$ \\
\hline SNB-75 & 1.164 & 2.099 & 1.941 & 1.614 & 1.244 & 1.316 & 0.918 & 83 & 48 & 9 & 16 & -21 & 8.83E-8 & 2.72E-5 & $>1.00 \mathrm{E}-4$ \\
\hline U251 & 0.429 & 2.109 & 2.035 & 1.603 & 0.948 & 0.841 & 0.659 & 96 & 70 & 31 & 25 & 14 & 3.23E-7 & $>1.00 \mathrm{E}-4$ & $>1.00 \mathrm{E}-4$ \\
\hline Melanoma & & & & & & & & & & & & & & & \\
\hline LOX IMVI & 0.305 & 2.041 & 1.921 & 1.262 & 0.802 & 0.401 & 0.449 & 93 & 55 & 29 & 6 & 8 & $1.56 \mathrm{E}-7$ & $>1.00 \mathrm{E}-4$ & $>1.00 \mathrm{E}-4$ \\
\hline MALME-3M & 0.832 & 2.375 & 2.363 & 1.881 & 1.791 & 1.733 & 1.503 & 99 & 68 & 62 & 58 & 43 & $3.65 \mathrm{E}-5$ & $>1.00 \mathrm{E}-4$ & $>1.00 \mathrm{E}-4$ \\
\hline M14 & 0.545 & 1.980 & 1.862 & 1.348 & 0.702 & 0.822 & 0.818 & 92 & 56 & 11 & 19 & 19 & $1.35 \mathrm{E}-7$ & $>1.00 \mathrm{E}-4$ & $>1.00 \mathrm{E}-4$ \\
\hline MDA-MB-435 & 0.575 & 2.576 & 2.501 & 0.610 & 0.355 & 0.514 & 0.323 & 96 & 2 & -38 & -11 & -44 & $3.09 \mathrm{E}-8$ & 1.11E-7 & $>1.00 \mathrm{E}-4$ \\
\hline SK-MEL-2 & 1.298 & 2.503 & 2.469 & 2.093 & 1.951 & 1.816 & 1.575 & 97 & 66 & 54 & 43 & 23 & $2.36 \mathrm{E}-6$ & $>1.00 \mathrm{E}-4$ & $>1.00 \mathrm{E}-4$ \\
\hline SK-MEL-28 & 0.553 & 1.762 & 1.694 & 1.288 & 1.167 & 1.159 & 0.907 & 94 & 61 & 51 & 50 & 29 & $1.02 \mathrm{E}-5$ & $>1.00 \mathrm{E}-4$ & $>1.00 \mathrm{E}-4$ \\
\hline SK-MEL-5 & 1.089 & 3.204 & 3.119 & 2.448 & 1.884 & 0.988 & 1.227 & 96 & 64 & 38 & -9 & 7 & $3.42 \mathrm{E}-7$ & & $>1.00 \mathrm{E}-4$ \\
\hline UACC-257 & 1.013 & 2.500 & 2.351 & 2.012 & 2.166 & 1.850 & 1.810 & 90 & 67 & 78 & 56 & 54 & > 1.00E-4 & $>1.00 \mathrm{E}-4$ & $>1.00 \mathrm{E}-4$ \\
\hline UACC- 62 & 0.918 & 2.598 & 2.390 & 1.594 & 1.362 & 1.185 & 1.038 & 88 & 40 & 26 & 16 & 7 & $6.21 \mathrm{E}-8$ & $>1.00 \mathrm{E}-4$ & $>1.00 \mathrm{E}-4$ \\
\hline Ovarian Cancer & & & & & & & & & & & & & & & \\
\hline & 0.342 & 1.972 & 1.885 & 1.373 & 0.832 & 0.623 & 0.487 & 95 & 63 & 30 & 17 & 9 & & & $>1.00 \mathrm{E}-4$ \\
\hline & 0.626 & 1.969 & 2.029 & 1.157 & 0.635 & 0.615 & 0.344 & 104 & 40 & 1 & -2 & -45 & & & $>1.00 \mathrm{E}-4$ \\
\hline & 0.761 & 2.129 & 2.077 & 1.824 & 1.321 & 1.029 & 1.0 & 96 & 78 & 41 & 20 & 18 & & & $>1.00 \mathrm{E}-4$ \\
\hline & 0.491 & 1.389 & 1.350 & 1.352 & 0.764 & 0.699 & 0.6 & 96 & 96 & 30 & 23 & 14 & & & \\
\hline OVCA & 0.738 & 3.003 & 2.973 & 2.775 & 1.738 & 1.424 & 1.6 & 99 & 90 & 44 & 30 & 39 & & $>1.0$ & $>1.00 \mathrm{E}-4$ \\
\hline NCI/ADR-I & 0.514 & 1.776 & 1.754 & 1.026 & 0.596 & 0.541 & 0.475 & 98 & 41 & 6 & 2 & -8 & & & $>1.00 \mathrm{E}-4$ \\
\hline SK-OV-3 & 1.344 & 2.662 & 2.605 & 2.372 & 2.061 & 1.976 & 1.789 & 96 & 78 & 54 & 48 & 34 & $4.78 \mathrm{E}-6$ & $>1.00 \mathrm{E}-4$ & $>1.00 \mathrm{E}-4$ \\
\hline Renal Cancer & & & & & & & & & & & & & & & \\
\hline & 0.678 & 2.647 & 2.517 & 2.199 & 1.313 & 0.872 & 0.756 & 93 & 77 & 32 & 10 & 4 & 4.03E-7 & $>1.00 \mathrm{E}-4$ & $>1.00 \mathrm{E}-4$ \\
\hline A498 & 1.770 & 2.414 & 2.298 & 1.805 & 1.580 & 1.545 & 1.35 & 82 & 5 & -11 & -13 & -23 & & $7 \mathrm{E}-7$ & $>1.00 \mathrm{E}-4$ \\
\hline ACHN & 0.4 & 2.06 & 2.005 & 1.6 & 1.158 & 0.7 & 0.6 & 96 & 75 & 43 & 19 & 13 & & $>1$. & $>1.00 \mathrm{E}-4$ \\
\hline CAK & 0.9 & 3.01 & 2.739 & 1.8 & 1.689 & 1.1 & 1.1 & 87 & 44 & 37 & 11 & 10 & & $>1$. & $>1.00 \mathrm{E}-4$ \\
\hline RXF 393 & 1.0 & 1.5 & 1.4 & 1.2 & 0.786 & 0.96 & 0.6 & 89 & 41 & -27 & -10 & -37 & & & $>1.00 \mathrm{E}-4$ \\
\hline SN12C & 1.3 & 3.283 & 3.19 & 3.12 & 2.494 & 1.7 & 1.9 & 96 & 92 & 60 & 19 & 34 & & $>1$. & $>1.00 \mathrm{E}-4$ \\
\hline TK-10 & 1.107 & 2.029 & 1.946 & 1.965 & 1.929 & 1.760 & 1.450 & 91 & 93 & 89 & 71 & 37 & & $>1.00 \mathrm{E}-4$ & $>1.00 \mathrm{E}-4$ \\
\hline บO-31 & 0.716 & 2.442 & 2.243 & 2.007 & 1.611 & 1.274 & 1.287 & 88 & 75 & 52 & 32 & 33 & $1.25 \mathrm{E}-6$ & $>1.00 \mathrm{E}-4$ & $>1.00 \mathrm{E}-4$ \\
\hline Prostate Cancer & & & & & & & & & & & & & & & \\
\hline & 0.835 & 1.973 & 1.964 & 1.665 & 1.100 & 0.993 & 1.025 & 99 & 73 & 23 & 14 & 17 & 2.89E-7 & $>1.00 \mathrm{E}-4$ & $>1.00 \mathrm{E}-4$ \\
\hline DU-145 & 0.453 & 1.737 & 1.839 & 1.643 & 0.683 & 0.517 & 0.527 & 108 & 93 & 18 & 5 & 6 & 3.72E-7 & $>1.00 \mathrm{E}-4$ & $>1.00 \mathrm{E}-4$ \\
\hline Breas & & & & & & & & & & & & & & & \\
\hline MCF7 & 0.480 & 2.360 & 2.10 & 1.112 & 0.832 & 0.651 & 0.81 & 91 & 34 & 19 & 9 & 18 & & $>1.0$ & \\
\hline MDA-MB-2 & 0.685 & 1.432 & 1.46 & 1.314 & 0.900 & 0.657 & 0.60 & 104 & 84 & 29 & -4 & -12 & 4.14 & 7.51 & $>1.00 \mathrm{E}-4$ \\
\hline HS 578T & 0.817 & 1.804 & 1.75 & 1.534 & 1.11 & 1.019 & 0.9 & 95 & 73 & 30 & 20 & 13 & 3.40 & $>1.00 \mathrm{E}-4$ & $>1.00 \mathrm{E}-4$ \\
\hline BT-549 & 1.257 & 2.273 & 2.167 & 1.916 & 1.813 & 1.459 & 1.471 & 90 & 65 & 55 & 20 & 21 & 1.37 & $>1.00 \mathrm{E}-4$ & $>1.00 \mathrm{E}-4$ \\
\hline T-47D & 1.008 & 2.612 & 2.459 & 1.971 & 1.797 & 1.472 & 1.720 & 90 & 60 & 49 & 29 & 44 & $8.39 \mathrm{E}-7$ & $>1.00 \mathrm{E}-4$ & $>1.00 \mathrm{E}-4$ \\
\hline MDA-MB-468 & 0.703 & 1.842 & 1.774 & 0.871 & 0.547 & 0.457 & 0.333 & 94 & 15 & -22 & -35 & -53 & $3.59 \mathrm{E}-8$ & $2.50 \mathrm{E}-7$ & $7.08 \mathrm{E}-5$ \\
\hline
\end{tabular}

